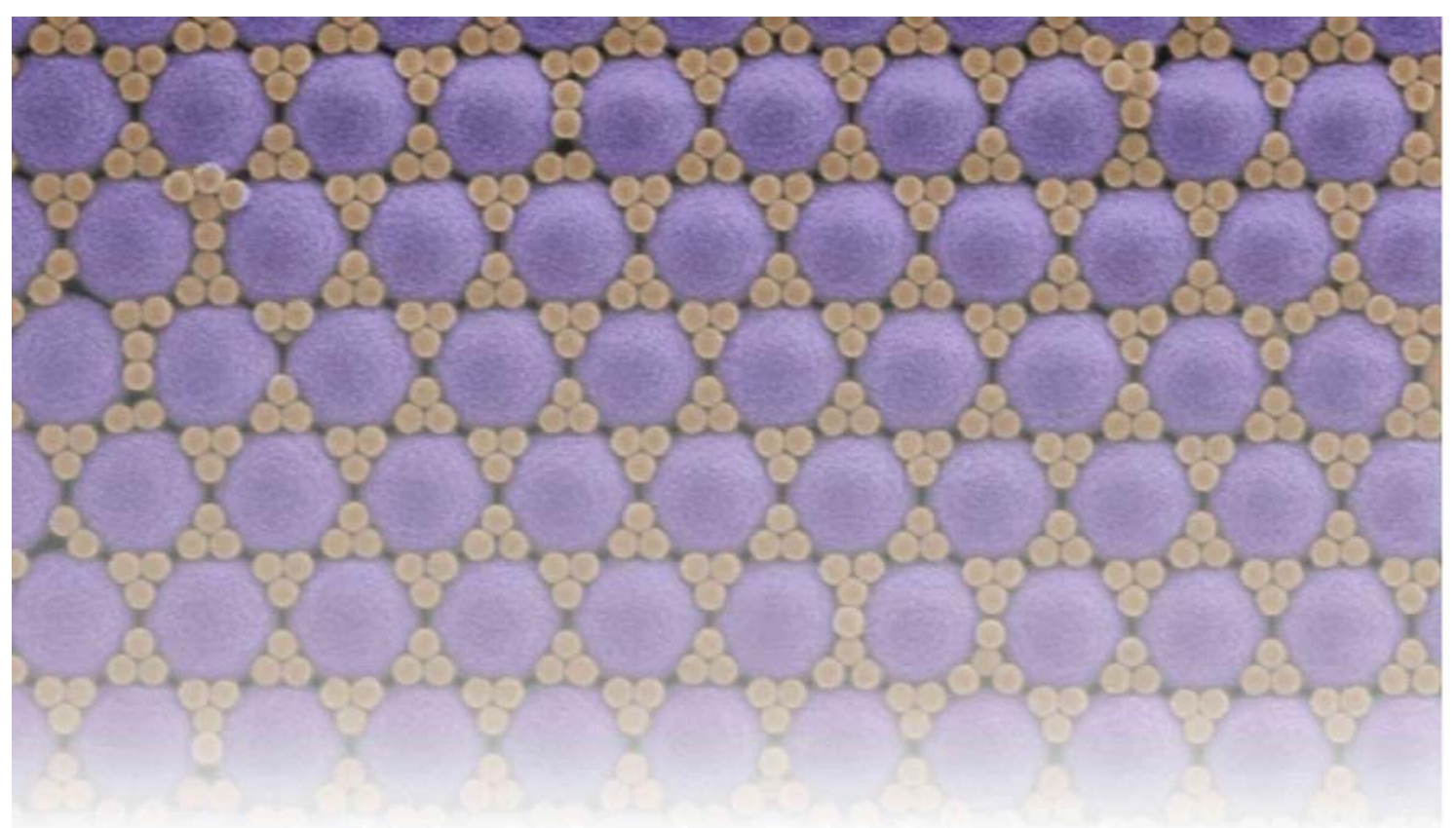

\title{
Surface Patterning with Colloidal Monolayers
}

Nicolas Vogel Dissertation

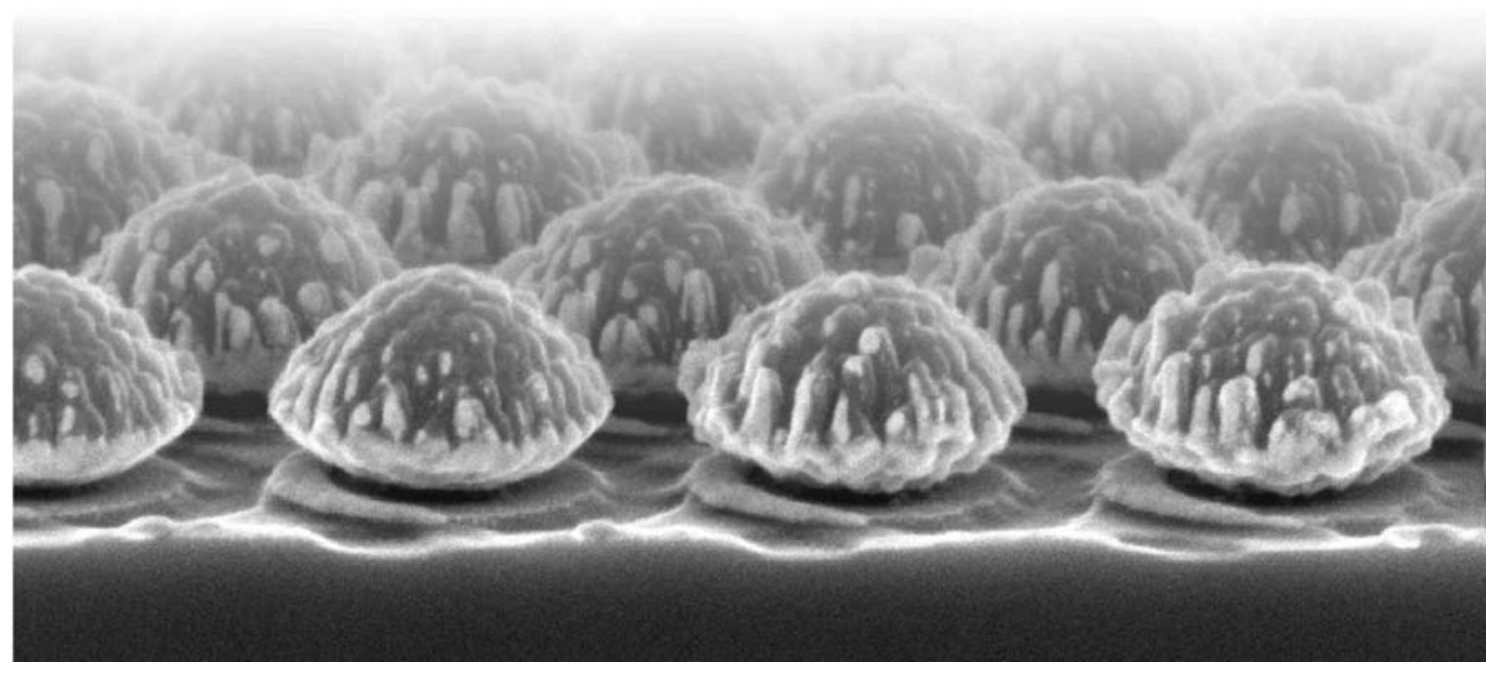





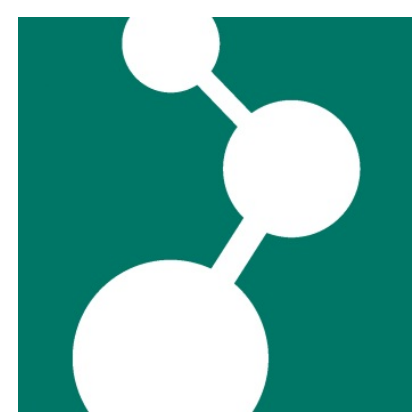

\title{
Surface Patterning with Colloidal Monolayers
}

\author{
Dissertation \\ zur Erlangung des Grades \\ „Doktor der Naturwissenschaften“ \\ im Promotionsfach Chemie \\ am Fachbereich Chemie, Pharmazie und Geowissenschaften \\ der Johannes Gutenberg-Universität Mainz.

\section{Nicolas Vogel} \\ Geboren in Mainz
}

Mainz 2011 

Die vorliegende Arbeit wurde im Zeitraum von August 2008 bis Juli 2011 am Max Planck Institut für Polymerforschung, Mainz und an der Foundation for Research and Technology Hellas, Heraklion, Griechenland angefertigt.

Dekan:

Erster Gutachter:

Zweiter Gutachter:

Tag der mündlichen Prüfung:
Prof. Dr. W. Hofmeister

Prof. Dr. Katharina Landfester

Prof. Dr. Michael Maskos 


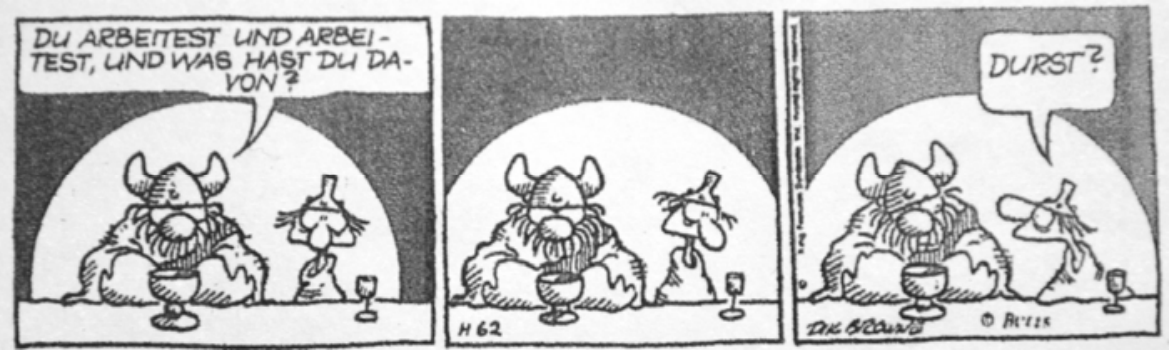




\section{Abstract}

This thesis focuses on the controlled assembly of monodisperse polymer colloids into ordered two-dimensional arrangements. These assemblies, commonly referred to as colloidal monolayers, are subsequently used as masks for the generation of arrays of complex metal nanostructures on solid substrates.

The motivation of the research presented here is twofold. First, monolayer crystallization methods were developed to simplify the assembly of colloids and to produce more complex arrangements of colloids in a precise way. Second, various approaches to colloidal lithography are designed with the aim to include novel features or functions to arrays of metal nanostructures.

The air/water interface was exploited for the crystallization of colloidal monolayer architectures as it combines a two-dimensional confinement with a high lateral mobility of the colloids that is beneficial for the creation of high long range order. A direct assembly of colloids is presented that provides a cheap, fast and conceptually simple methodology for the preparation of ordered colloidal monolayers. The produced two-dimensional crystals can be transformed into nonclose-packed architectures by a plasma-induced size reduction step, thus providing valuable masks for more sophisticated lithographic processes. Finally, the controlled co-assembly of binary colloidal crystals with defined stoichiometries on a Langmuir trough is introduced and characterized with respect to accessible configurations and size ratios.

Several approaches to lithography are presented that aim at introducing different features to colloidal lithography. First, using metal-complex containing latex particles, the synthesis of which is described as well, symmetric arrays of metal nanoparticles can be created by controlled combustion of the organic material of the colloids. The process does not feature an inherent limit in nanoparticle size and is able to produce complex materials as will be demonstrated for FePt alloy particles. Precise control over both size and spacing of the particle array is presented. Second, two lithographic processes are introduced to create sophisticated nanoparticle dimer units consisting of two crescent shaped nanostructures in close proximity; essentially by using a single colloid as mask to generate two structures simultaneously. Strong coupling processes of the parental plasmon resonances of the two objects are observed that are accompanied by high near-field enhancements. A plasmon hybridization model is elaborated to explain all polarization dependent shifts of the resonance positions. Last, a technique to produce laterally patterned, ultra-flat substrates without surface topographies by embedding gold nanoparticles in a silicon dioxide matrix is applied to construct robust and re-usable sensing architectures and to introduce an approach for the nanoscale patterning of solid supported lipid bilayer membranes. 


\section{Table of contents}

1 Introduction $\quad 1$

2 Motivation and Outline 3

3 Theory 5

3.1 Synthesis of polymeric colloidal particles 5

3.1.1 Miniemulsion polymerization 5

3.1.2 Emulsion polymerization

3.1.3 Summary and comparison between miniemulsion and emulsion polymerization $\quad 9$

3.2 Forces at work in colloidal stability and crystallization $\quad 10$

$\begin{array}{ll}3.2 .1 \text { Colloid stability in aqueous dispersions } & 10\end{array}$

3.2.1.1 Van der Waals interactions 10

3.2.1.2 Electrostatic interactions 12

$\begin{array}{ll}\text { 3.2.1.3 DLVO theory } & 14\end{array}$

3.2.2 Colloids at interfaces $\quad 15$

$\begin{array}{ll}\text { 3.2.2.1 Why particles are trapped at an interface } & 15\end{array}$

$\begin{array}{ll}3.2 .2 .2 \text { Dipole interactions } & 18\end{array}$

$\begin{array}{ll}3.2 .2 .3 \text { Capillary forces } & 19\end{array}$

3.3 Two-dimensional colloidal crystallization 20

3.3.1 Close-packed colloidal monolayers 20

3.3.1.1 Direct assembly methods 22

3.3.1.2 Liquid interface-mediated methods $\quad 27$

3.3.1.3 Summary of the crystallization methods $\quad 30$

3.3.2 Non-close-packed monolayers 32

3.3.2.1 Non-close-packed architectures from close-packed monolayers 32

3.3.2.2 Non-close-packed monolayers by spin coating 33

3.3.2.3 Direct assembly of non-close-packed monolayers at the oil-water interface $\quad 34$

3.3.3 Binary colloidal monolayers $\quad 35$

3.3.3.1 Sequential assembly of binary monolayers 36

3.3.3.2 Co-assembly of binary monolayers $\quad 38$

3.4 Preparation of nanostructures on surfaces 39

3.4.1 Colloidal lithography 41

3.4.2 Micellar lithography $\quad 41$ 
3.4.3 Non-conventional colloidal lithography

3.4.4 Applications of colloidal monolayers and surface nanostructures fabricated by colloidal lithography

3.5 Localized surface plasmon resonances: a fundamental properties of metallic nanostructures

3.5.1 Localized surface plasmon resonances

3.5.2 Sensing with localized surface plasmons

3.5.3 Plasmon hybridization

\section{Characterization methods}

4.1 Atomic force microscopy (AFM)

4.2 Inductively coupled plasma optical emission spectrometry (ICP-OES)

4.3 Two-dimensional chemistry and physics on a Langmuir trough

4.4 Scanning electron microscopy (SEM)

4.5 Spinning drop tensiometry

\section{Results and Discussion}

5.1 Particle synthesis and characterization

5.1.1 Accurate determination of metal-containing polymer latexes using inductively coupled plasma - optical emission spectrometry

5.1.2 Synthesis of metal-complex containing latex particles

5.1.2.1 Introduction

5.1.2.2 Incorporation of metal complexes by emulsion polymerization

5.1.2.3 Reasons for the different behavior of the metal complexes in incorporation reactions

5.1.2.4 Incorporation of metal complexes by miniemulsion polymerization

5.1.2.5 Seeded emulsion polymerization as a tool to control the size of polymeric nanoparticles

5.2 Assembly of colloidal monolayer architectures

5.2.1 Convenient production of close-packed monolayers using direct assembly at the air/water interface

5.2.2 Non-close-packed monolayers by plasma induced size reduction

5.2.3 Ordered binary monolayers with adjustable stoichiometries 
5.3.1.1 Introduction

150

5.3.1.2 Platinum nanoparticle arrays

5.3.1.3 FePt alloy nanoparticle arrays

5.3.2 Plasmonic structures from non-close packed monolayers 159

$\begin{array}{ll}\text { 5.3.2.1 Arrays of gold nanocrescents } & 160\end{array}$

$\begin{array}{ll}\text { 5.3.2.2 Stacked double crescent arrays } & 165\end{array}$

5.3.2.3 Opposing double crescents with tunable plasmon resonances $\quad 179$

5.3.3 Laterally patterned, ultraflat substrates by colloidal lithography and their applications

5.3.3.1 Introduction: laterally patterned, ultraflat substrates 194

5.3.3.2 Re-usable sensing architectures 196

5.3.3.3 Nanoscale patterning of solid supported membranes by integrated diffusion

$\begin{array}{ll}\text { barriers } & 202\end{array}$

6 Experimental Section $\quad 212$

6.1 General procedures 212

$\begin{array}{ll}6.2 \text { Materials } & 215\end{array}$

6.3 Experimental details for the individual chapters 215

7 Summary and Outlook 233

8 List of Abbreviations $\quad 238$

9 Acknowledgements $\quad 239$

10 Curriculum Vitae and List of Publications 241

11 Bibliography $\quad 244$ 


\section{Introduction}

"There's plenty of room at the bottom!" ${ }^{, 1]}$ With his famous exclamation, Richard Feynman encouraged scientists to set sail to discover and reveal the secrets of a new world: the nanoscale. 2011 - fifty-two years after setting sail, scientists have not only found land where expected, we have also learned to live in this small world, where gravity is a mere spectator while other forces are on the field, where gold can be red or blue or purple, honey does not necessarily flow slower than water and nobody knows whether the cat is dead or alive.

Yet, even though enormous progress has been made in understanding physical effects and properties of matter at the nanoscale, we are still incredibly clumsy as construction workers. We have been able to build sophisticated equipment that can produce feature sizes of several nanometers and, in some cases, we even manage to deposit single atoms in a controlled way. Still, these approaches seem unnecessarily cumbersome when compared to the emergence of incredibly complex structures in nature. Maybe the most fundamental principle we have started to adapt from nature is self-assembly. George Whitesides rhetorically asked "Will we ever be able to put parts of a computer into a bucket, shake gently, and see a computer spontaneously emerge?" and gave the answer himself: "Not soon; but even small steps in the direction of selfassembly could save enormous efforts by humans and machines." ${ }^{\text {,[2] }}$

Though we cannot assemble a computer in a way that nature assembles a cell, we have started to explore the possibilities of using simple materials capable of forming ordered structures by self-assembly processes to produce more complex, even functional structures.

As a reply to Richard Feynman, my colleague Uli Jonas used to nonchalantly state that "there is even more room in the first floor" when referring to assembly processes at the mesoscale. This length-scale of approximately $100-1000 \mathrm{~nm}$ can be considered as the transition between the nanoscale and our familiar micro- and macroscopic world. At this scale, enormous progress has been made in the controlled assembly and design of structures and a variety of applications have found entry into daily life, e.g., to prevent dirt from attaching to facades, glasses from fogging and reflecting light and to create colorful surfaces without applying a single dye molecule.

Colloidal particles have long been recognized as valuable building blocks for the construction of structures at the mesoscale. They are cheap yet precise to design and are able to self-assemble into well-ordered formations in two- or three dimensions.

The game is as old as the author of this thesis: Let colloids assemble into a two-dimensional lattice and use the resulting monolayer as a mask to create surface patterns at the nanoscale. 30 years after Pieranskis first report on two-dimensional colloid crystallization ${ }^{[3]}$ and Fischers idea 
to use a colloid monolayer as lithographic mask, ${ }^{[4]}$ the subject is far from being old news. When Richard van Duyne demonstrated the preparation of triangular noble-metal nanoparticles in the 1990 's, ${ }^{[5-6]}$ the stage was set for colloidal lithography to take part in what has remained one of the most intriguing properties of nanoparticles: the excitation of collective electron oscillations known as plasmon resonances. ${ }^{[7-8]}$

Until now, the field of colloidal lithography has remained very active and increasingly complex structures have been- and are continued to be prepared in nanoscale dimensions, ranging from rings, discs and crescent-shaped particles to bowls, wells, or needles. ${ }^{[9-10]}$

A plethora of applications have been demonstrated for colloidal lithography-based structure arrays that exploit different properties inherent to the process. The plasmonic properties of noble-metal nanoparticles have been extensively used to detect binding events; e.g. of biologically relevant molecules and the strong near-fields arising at resonance wavelengths continue to be used to enhance dye fluorescence ${ }^{[11]}$ or Raman signals. ${ }^{[12]}$ The high symmetry and nanoscale periodicity of the arrays has been applied to mimic the structural color of butterfly wings, ${ }^{[13]}$ create super-hydrophobic ${ }^{[14]}$ and self-cleaning surfaces, ${ }^{[15]}$ and to control the adhesion of cells. ${ }^{[16]}$

Even after all of these achievements, the question remains: What else can we do? In principal, the combination of a top-down patterning process with bottom up approaches to functionalize these structures in order to yield a desired function leaves plenty of room for imagination and creativity. We can apply different materials, produce larger, smaller or more complex structures, create sharp edges or soft curvatures, assemble in three-dimensions or drill into the substrate, introduce hierarchy or responsive functions. Or, in other words, there is still plenty of room above, below and around a colloidal monolayer. As George Whitesides put it: "Our primary interest in nano, right now, is in fabrication. My argument for focusing on fabrication is that since I don't know what nano is going to be, to the extent that our efforts can open nanostructures, make nanostructures of whatever sort accessible to the widest possible variety of scientists in the easiest possible way, this is, to me, a good contribution to make." $"[17]$ 


\section{Motivation and Outline}

To continue along the lines of thoughts of George Whitesides, the primary focus of this thesis is on fabrication or, to be more precise, the generation of symmetric surface patterns via colloidal crystallization. As such, the self-assembly process of colloidal particles to form highly ordered, two-dimensional crystals is at the heart of the projects introduced in this thesis.

As briefly touched on in the introduction, colloidal self-assembly offers several attractive benefits for the creation of surface patterns. First, colloids mostly consist of cheap materials and can be synthesized with enormous precision with respect to their size and size distribution. Second, they are able to assemble into two-dimensional arrays with an astonishingly high overall order that can subsequently be transferred to nanostructure arrays when the monolayer is used as a mask in a lithographic process. As the colloids can be synthesized in a broad size range down to several tens of nanometers, the diffraction limit is conveniently circumvented and structure arrays with feature sizes as small as several nanometers can be created. This lithographic process does not require any sophisticated equipment. Compared to electron beam lithography or a focused ion beam, both of which are able to create arbitrary structures with similar resolution, colloidal lithography is a parallel process in which all structures are created simultaneously during the lithographic process. In contrast, both other techniques mentioned are serial processes that literally "write" one structure after another. Hence, colloidal lithography is both cheaper and faster - and in principal amendable to arbitrary large surfaces.

One of the main drawbacks of the colloidal lithography process is its inherent restriction to limited structural designs that are pre-set by the arrangements of the colloids in the monolayer. One major research direction is therefore the extension of accessible structures that can be created using colloids as masks. Originally, triangular structures that reflect the shape of the interstitial sites of a densely-packed monolayer were at the forefront of colloidal lithography and an immense amount of work has been designated to investigate and exploit their plasmonic properties; especially in sensing applications.

Only in the last few years has the field widened to target more complex organizations of colloids. First, the controlled creation of non-close-packed monolayers was a milestone for applications in lithography as the area shaded by the colloids could be used in lithographic processes by introduction of rotations and angular evaporation and etching steps. Thus, a variety of different surface structures are now available in symmetric arrays by colloidal lithography. On the other hand, several approaches have been introduced for the assembly of binary crystals consisting of a close-packed monolayer of large spheres with small spheres present in the interstitial sites formed by the large spheres. As both number and size of the small spheres can 
be adjusted separately, binary monolayers possess a rich variety of possible conformations and crystal structures compared to single particle monolayers.

The motivation of this thesis is to further contribute to flexibility and versatility of colloidal lithography. Several methods for the creation of colloidal crystals with different complexities are presented and subsequently used in lithographic approaches to introduce novel structural features or functions to nanostructure arrays. Even though several applications for the structured arrays prepared in this thesis can be envisioned and are briefly touched on in the individual chapters, the aim of the work is not the detailed exploitation of the structures in sophisticated applications. Instead, structures and fabrication processes are presented and made available to a broad range of scientists by the simplicity of the colloidal crystallization approach.

The thesis is organized as follows. First, a short survey about the theoretical foundations of processes relevant to this thesis is presented (chapter 3). These involve the synthesis of colloids, forces involved in their stabilization and crystallization and experimental methods to yield twodimensional crystals. Furthermore, a comparison of colloidal lithography to other nanopatterning methods is given along with a short introduction on the interaction of metal nanostructures with light that lead to a resonance phenomenon called plasmon resonances. Following the theoretical section will be a short introduction to methods that have been used in this thesis (chapter 4).

The results and discussion chapter is generally divided into three parts: synthesis of functional colloidal particles, monolayer crystallization processes and different lithographic approaches. The three main parts are further divided into subchapters that present the individual projects that are subject of this thesis. As several projects require synthesis as well as crystallization and lithography, the different chapters are generally interconnected. The unifying motive for all projects is the use of colloidal particles to generate symmetric structure arrays. 


\section{Theory}

\subsection{Synthesis of polymeric colloidal particles}

All colloids prepared in this thesis were synthesized by either miniemulsion or surfactant free emulsion polymerization. In the next chapters, the mechanisms, differences and benefits of the two types of heterophase polymerizations will be discussed.

\subsubsection{Miniemulsion polymerization}

A miniemulsion is a heterophase system that consists of small droplets $(\sim 30-500 \mathrm{~nm})$ of one liquid in an (inmiscible) other. One may distinguish direct miniemulsions (oil in water) and indirect miniemulsions (water in oil). ${ }^{[18-19]}$ Only direct miniemulsions will be discussed here as they were exclusively applied in this thesis. The miniemulsion is created by applying high shear forces (e.g. ultrasound or high pressure homogenization) ${ }^{[18-20]}$ to a two phase mixture of oil (in a polymerization process typically the monomer) and water (Figure 3.1.1).
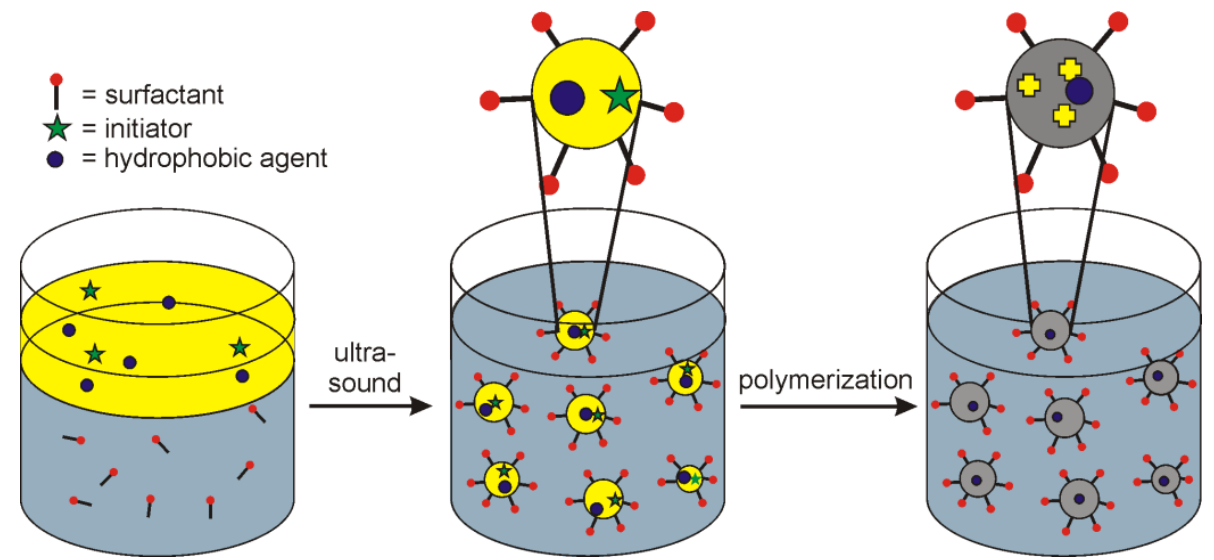

Figure 3.1.1. Schematic representation of the miniemulsion process.

Though a miniemulsion is not in the thermodynamic equilibrium state, the droplets are critically stabilized. This stabilization requires the addition of two types of supplementary molecules to the emulsion. The water phase contains surfactant molecules while a second stabilization agent (costabilizer), termed ultrahydrophobe is added to the oil phase. The ultrahydrophobe is by definition the least soluble substance in the water phase. Two degradation mechanisms exist for a non-stabilized emulsion: collision and subsequent coalescence of individual droplets and Ostwald ripening (Figure 3.1.2). ${ }^{[21-22]}$ The latter describes the growth of larger droplets on the 
account of smaller ones by diffusion of oil-phase molecules through the water phase. This is caused by the higher Laplace pressure in the smaller droplets that is defined as the difference of pressure $(\Delta P)$ between the inside of a droplet $\left(P_{\text {inside }}\right)$ compared to the outside $\left(P_{\text {outside }}\right)$ and is given by the following relation:

$$
\Delta P=P_{\text {inside }}-P_{\text {outside }}=\frac{\gamma}{R}
$$

As can be seen from the equation, the Laplace pressure is proportional to the surface tension of the liquid $\gamma$ and inverse proportional to the radius of the droplet $R$.

In a miniemulsion, both degradation mechanisms are effectively counteracted. Coalescence of individual droplets is prevented by steric- or electrostatic repulsion caused by the surfactant molecules present at the droplet surface. The addition of the ultrahydrophobe induces an osmotic pressure that counteracts the Laplace pressure responsible for Ostwald ripening processes. Figure 3.1.2 schematically illustrates the process. Without an ultrahydrophobe, the higher Laplace pressure leads to diffusion of monomer from smaller droplets to larger ones (a) until the emulsion is degraded. The presence of ultrahydrophobic molecules in the droplets (symbolized as blue dots in Figure 3.1.2) counteracts the ripening process (b): if a monomer molecule diffused from a smaller to a larger droplet, the concentration of ultrahydrophobe in the smaller droplets would increase. This imbalance of concentration would than create an osmotic pressure difference that induced a backwards diffusion towards the smaller droplet.

In a system with ultrahydrophobe, the two pressures are in equilibrium and Ostwald ripening is prevented.

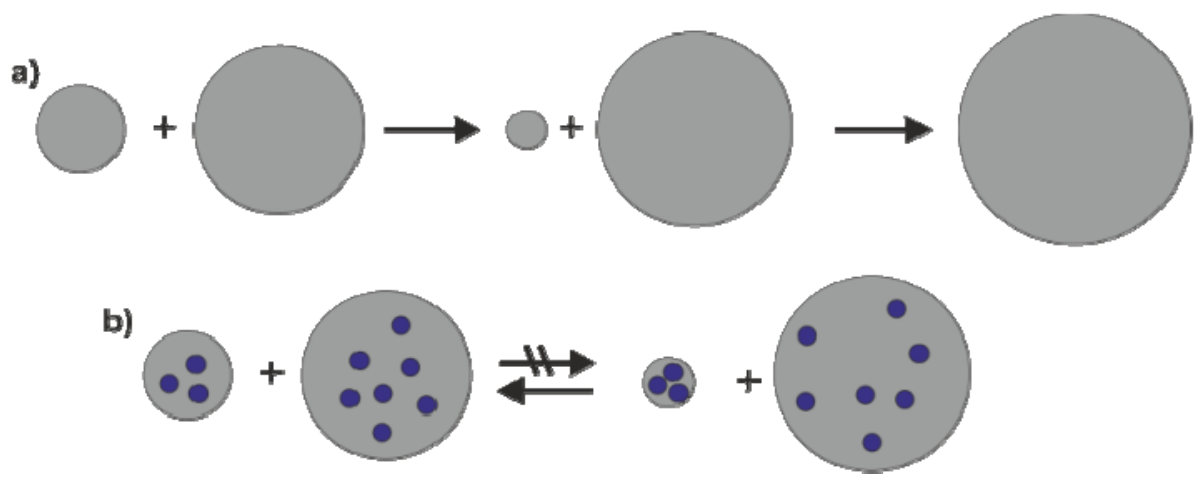

Figure 3.1.2. Degradation of an emulsion by Ostwald ripening and prevention of the process in a miniemulsion. a) Ostwald ripening leads to the growths of bigger particles on the account of the smaller particles. b) By addition of an ultrahydrophobe, an osmotic pressure is build up upon diffusion that prevents the ripening process. 
To prepare latex particles by radical polymerization in miniemulsion, an initiator is added to the two phase system with monomer molecules as the dispersed phase. Both, initiation from the water and from the oil droplets is possible. After miniemulsification, polymerization of the monomer droplets is readily achieved, e.g. by increasing the temperature.

The unique feature of the miniemulsion process is the integrity of the droplets during the polymerization process. In other words, as no net-diffusion takes place, the miniemulsion can be regarded as a compartmentalization of the monomer phase. Any functional molecule initially added to the latter will be statistically distributed in the miniemulsion droplets. Each droplet acts as an individual entity during polymerization (nanoreactor concept). ${ }^{[1-22]}$ Thus, the composition of the final latex particles resembles the composition of the monomer phase. As no diffusion takes place, the polymerization kinetics resembles the kinetics of a bulk radical polymerization. The integrity of the droplets due to the nanoreactor concepts makes miniemulsion a powerful tool for the incorporation of defined amounts of functionalities into latex particles and a rich variety of different substances has been successfully incorporated; ${ }^{[19-20,23]}$ including fluorescent dyes, ${ }^{[24]}$ pharmaceutically active substances, ${ }^{[25]}$ metal complexes ${ }^{[26]}$ and inorganic nanoparticles. ${ }^{[27]}$

A further benefit of miniemulsion polymerization is the high versatility towards monomers and polymerization types that can be applied. As the process can be conducted as a direct (oil in water) and indirect (water in oil) process, both hydrophobic and hydrophilic monomers can be polymerized. The process is not limited to radical polymerization mechanisms; step-growth reactions as well as anionic polymerizations can be realized as well. ${ }^{[18]}$

\subsubsection{Emulsion polymerization}

Emulsion polymerization can be considered as the standard preparation technique for latex particles. Since the first report by William Harkins in $1947,,^{[28]}$ emulsion polymerization has been extensively investigated and applied. In contrast to miniemulsion polymerization, emulsion polymerization processes entirely rely on diffusion. Schematic illustrations of the mechanisms for the classical emulsion polymerization process ${ }^{[28]}$ as well as the surfactant-free emulsion polymerization process introduced by Goodwin and Ottewill ${ }^{[29]}$ and employed in this thesis are shown in Figure 3.1.3. In both cases, a monomer with a low but finite solubility in the continuous water phase is stirred to created macroscopic droplets. In (a), the classical emulsion polymerization reaction is shown: the presence of surfactant molecules above the critical micelle concentration leads to the formation of surfactant micelles that are filled with monomer by diffusion. The polymerization is initiated from the water phase where the initiator 
decomposes and starts to add monomer molecules present in small amounts. Eventually, the oligo-radicals formed in the water phase enter a micelle and polymerize in the monomer-rich environment. Further monomer is supplied by diffusion to the micelles until all monomer is consumed. For statistical reasons, the primary loci of polymerization are not the monomer droplets but the micelles (as they exist in an extremely high number). Figure 3.1.3b schematically illustrates the surfactant-free emulsion polymerization process. In contrast to the classical method, no surfactant is added and surface-active species are formed in situ by the charged initiator molecules and the presence of water-soluble co-monomers (Figure 3.1.4). After initiation in the water phase, the radical adds monomer units until it reaches a critical chain length (depicted as $\mathrm{M}_{\mathrm{i}} \cdot$ ) upon which it becomes too hydrophobic to be soluble in the water phase. Thus, it collapses into a globule and forms a particle nucleus. Several of these nuclei subsequently assemble together to form a stable primary particle that is stabilized by charges of initiator and comonomer molecules. This primary particle then acts as the polymerization locus as monomer diffuses into the hydrophobic compartment and is subsequently polymerized to a latex particle. The mechanisms of the types of emulsion polymerization have been examined in a very detailed way by Harkins ${ }^{[28]}$ and Hansen and Ugelstad $^{[30-31]}$ respectively.
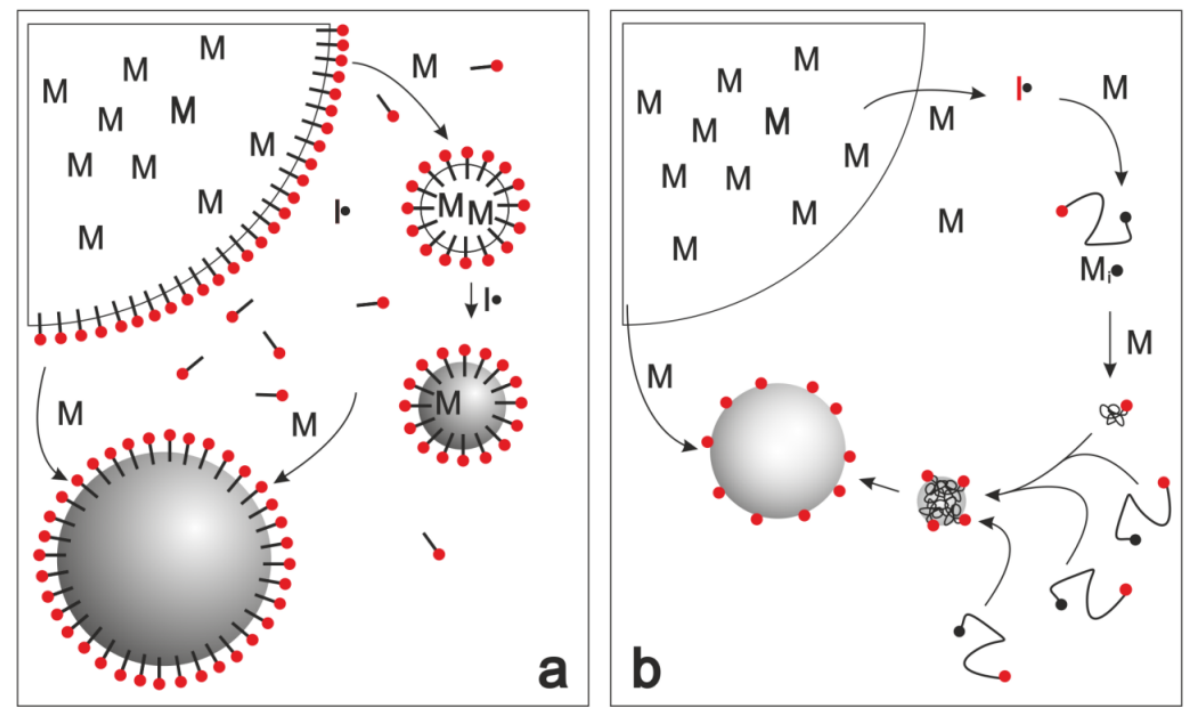

Figure 3.1.3. Schematic representation of the emulsion polymerization process. a) Classical emulsion polymerization process with surfactant molecules above the critical micelle concentration. b) Surfactantfree polymerization process. 
<smiles>C=Cc1ccc([N+](=O)[O-])cc1</smiles>

NapSS<smiles>C=CC(=O)O</smiles>

AA

Figure 3.1.4. Typical comonomers used in surfactant-free emulsion polymerization. Sodium para-styrene sulfonate (NapSS) and acrylic acid (AA)

\subsubsection{Summary and comparison between miniemulsion and emulsion polymerization}

Emulsion polymerization; especially the surfactant-free variation is capable of producing latex particles with an extremely narrow size distribution. This makes them attractive for colloidal crystallization and applications where a high degree of order is required, most prominently in photonic and lithographic applications. In contrast to miniemulsion polymerization, the incorporation of additional, functional molecules is rather limited as the composition of the final latex particles does not resemble the composition of the monomer phase due to differences in diffusion between the different molecules. Additionally, the choice of monomers in emulsion polymerization is more limited and usually restricted to radical polymerization of styrene and acrylates. In summary, emulsion polymerization is the method of choice if applications demand for plain spheres with a high degree of monodispersity while miniemulsion is preferred if more sophisticated systems regarding polymer type, latex architectures and functionalizations are needed. Table 3.1.1 summarizes the different properties of emulsion and miniemulsion polymerization.

Table 3.1.1. Comparison between the miniemulsion and the emulsion polymerization process.

\begin{tabular}{l|l|l}
\hline & Miniemulsion Polymerization & Emulsion Polymerization \\
\hline Polymerization type & Radical, anionic, polyaddition,... & Limited to radical \\
State of initial emulsion & Critically stabilized miniemulsion & Macroemulsion \\
Emergence of particles & Preformed & Diffusion controlled \\
Composition of final latex & As initial monomer phase & Different from monomer phase \\
Particle sizes & $\sim 30-300 \mathrm{~nm}$ & $\sim 50-800 \mathrm{~nm}$ \\
Choice of monomer & Variable & Limited \\
Incorporation of functionalities & Straightforward & Limited \\
Polydispersity of latex particles & Medium & Extremely low \\
\hline
\end{tabular}




\subsection{Forces at work in colloidal stability and crystallization}

The stability of a colloidal system is the dominating issue for the fundamental understanding of dispersed matter and its importance reaches far into every-day life. As very common examples, one might mention the coagulation of erythrocytes in wound-closing processes, the removal of dirt in laundry processes as well as the stability of beer foam.

The colloidal stability is governed by a balance between attractive van der Waals forces and repulsive forces that can be of electrostatic and steric nature. Here, only electrostatic stabilization is considered. The combinations of these forces sums up to a complete interaction potential for two spheres that is described by the DLVO theory which will be introduced in the next chapter.

In crystallization processes, especially in two dimensions, another force has to be considered as the colloidal particles are - in the majority of all cases - deposited from a liquid dispersion onto a solid substrate. Upon drying, the water level eventually reaches a height that is comparable to the colloids' dimensions. As a consequence, the liquid film around the particles deforms and capillary forces arise. Such forces have to be considered in models to describe crystallization processes. The same capillary forces also act on particles on the air/water surface. The changes in the interaction potential of colloids due to the presence of an interface will therefore be discussed below.

\subsubsection{Colloid stability in aqueous dispersions}

The combination of van der Waals attraction and electrostatic repulsion into an interaction potential to describe the conditions of colloidal stability has been introduced independently by Derjaguin and Landau ${ }^{[32]}$ and Verwey and Overbeek ${ }^{[33]}$ and became known as DLVO theory. The individual forces will be introduced briefly before the complete interaction potential will be presented and discussed. A more detailed discussion of the individual components can be found for example in textbooks of Butt ${ }^{[34]}$ and Tadros ${ }^{[35]}$.

\subsubsection{Van der Waals interactions}

At short distances, molecules attract each other due to intermolecular dipole interaction. These dipoles can be of permanent character (i.e. in polar molecules) or induced in non-polar 
molecules due to the polarizability of the electron clouds. Three distinct contributions can be distinguished: ${ }^{[34]}$ dipole-dipole (Keesom), dipole-induced dipole (Debye) and induced dipoleinduced dipole (London). All these forces are summarized as van der Waals interactions $U_{v d W}$. They are all inherently attractive and inversely proportional to the sixth power of the interatomic distance $d$ :

$$
U_{v d W}=U_{\text {Keesom }}+U_{\text {Debye }}+U_{\text {London }}=-\frac{C_{\text {orient }}}{d^{6}}-\frac{C_{\text {induced }}}{d^{6}}-\frac{C_{\text {disp }}}{d^{6}}=-\frac{C_{A B}}{d^{6}}
$$

$C_{A B}$ is a constant that includes all distance-independent terms of the individual contributions of two molecules A and B, most importantly the polarizability of the molecules and their dipole moment.

For mesoscopic colloids, two approaches exist for the determination of the van der Waals interaction energy. The microscopic approach sums up the molecular forces while the macroscopic approach, known as Lifshitz theory ${ }^{[36]}$ calculates the energy by macroscopic material properties and includes effects of the medium in between the colloids.

In the (more descriptive) microscopic approach, the van der Waals interaction energy of two planes, $U_{\text {plane, }}$ is calculated by summing up the van der Waals energy between all molecules of solid A and solid B. A mathematical treatment, as described in textbooks ${ }^{[34]}$ yields for the van der Waals energy per unit area $u_{\text {plane: }}$ :

$$
u_{\text {plane }}=\frac{U_{\text {plane }}}{A}=-\frac{\pi \rho_{A} \rho_{B} C_{A B}}{12 D^{2}}=-\frac{A_{H}}{12 \pi D^{2}}
$$

In the equation, $\rho_{A}$ and $\rho_{B}$ are the molecular densities of the two solids $\mathrm{A}$ and $\mathrm{B}, D$ is the distance between the two planes and $C_{A B}$ is the sum of the molecular contributions. All constants can be combined into the Hamaker constant $\left(A_{H}=\pi^{2} \rho_{A} \rho_{B} C_{A B}\right)$ that describes the interactions between the two different materials.

Similarly, the interaction energy between two spheres with the radii $R_{1}$ and $R_{2}$ can be calculated and simplified by the Derjaguin approximation. The latter simplifies the distance dependence of two spheres by the distance between two plates if the distance $D$ is substantially smaller than the radii $R_{i}$ of the spheres. The approximation yields:

$$
U_{\text {sphere-sphere }}=-\frac{A_{H}}{6 D} \cdot \frac{R_{1} R_{2}}{\left(R_{1}+R_{2}\right)}=-\frac{A_{H} R}{12 D}
$$




\subsubsection{Electrostatic interactions}

Charged surfaces, occurring either by chemical manipulation of the surface properties, adsorption of ions (e.g. on metal surfaces) or by dissociation of surface groups (e.g. metal oxide surfaces) cause an electric field that attracts counter-ions to form an electric double layer. Several theories to describe the distribution of charges in this layer have been established. Helmholtz proposed a model that consists of a charged surface and a layer of rigidly adsorbed solvated ions from the aqueous electrolyte solution. As a result, the surface potential decreases linearly from the electrode. Gouy-Chapman modeled the ion distribution as a diffuse double layer characterized by an excess of counter-ions in the vicinity of the electrode charges. Due to thermal motion, the counter-ions are not rigidly fixed to the electrode but are able to move away from the electrode against the electric potential.

Stern combined the existing theories into a model that features a rigid layer of adsorbed counterions in close vicinity of the charged surface and a diffuse layer of non-bound ions at higher distances of the surface. ${ }^{[37]}$ Figure 3.2 .1 a shows the model schematically. In the rigidly adsorbed Stern-layer, the surface potential decreases linearly with distance. The dependency changes into an exponential decay in the diffuse layer where an excess of counter-ions decreases the surface potential to the potential of the bulk electrolyte solution. The complete distance dependence of the potential is shown in Figure 3.2.1b. The Stern layer can either be modeled as a single layer of hydrated counter-ions or be further divided into two regions: the inner and outer Helmholtz layer. The inner Helmholtz layer consists of oriented water molecules and like-charged ions that are strongly bound to the surface by van der Waals forces or specific interactions with the surface material while the outer Helmholtz layer hosts the hydrated counter-ions. Figure 3.2.1c depicts the more complex model of the Stern layer.

The shear plane is defined as the layer of ions that is attracted strong enough to the surface that closely follow a movement of the surface (e.g. of a colloidal particle). The potential at the distance of the shear plane is called $\zeta$-potential. It does not necessarily correspond to the Stern potential $\Psi_{o}$ as at high electrolyte concentrations, the diffuse layer is more and more compressed. Thus, a lower potential at the shear plane is detected even though no changes in the Stern layer occur (Figure 3.2.1d). 


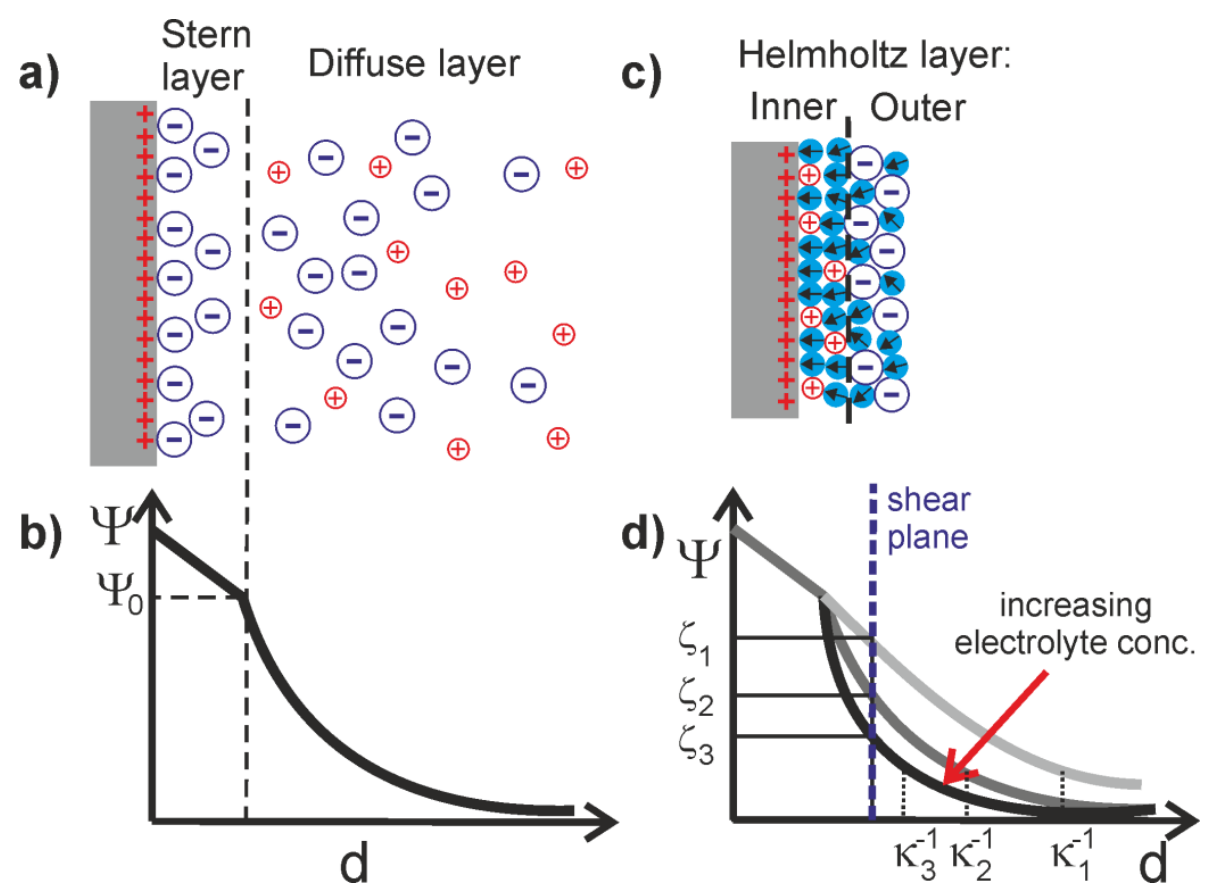

Figure 3.2.1. Stern model of the electric double layer. a) Schematic illustration; b) electrostatic potential versus distance diagram. c) More complex model of the Stern layer with inner and outer Helmholtz layer.

d) Effect of increasing electrolyte addition; affecting both the $\zeta$-potential and the Debye length $\kappa^{-1}$.

Mathematically, the exponential decay in the Stern model can be described by the PoissonBoltzmann equation. Here, the ion distribution around the surface resulting from electrostatic interaction and thermal motion are described by the Boltzmann distribution and the directing potential of the fixed charges at the surface is captured by the Poisson equation. ${ }^{[34,38]}$ Neglecting the contribution of the Stern layer and linearizing of the Poisson-Boltzmann equation ${ }^{[34]}$ yields a simple expression for the electrostatic interaction potential $\Psi$ in dependence of the distance $d$ :

$$
\begin{aligned}
& \Psi(D)=\Psi_{0} \cdot \exp (-\kappa d) \\
& \kappa=\sqrt{\frac{2 c_{0} e^{2}}{\varepsilon \varepsilon_{0} k_{B} T}}
\end{aligned}
$$

In the formula, $\kappa$ combines all constants $\left(c_{0}\right.$ as the ion concentration; $\varepsilon, \varepsilon_{0}$ the dielectric constants of the bulk electrolyte solution and of vacuum, respectively; and $k_{B}$ the Boltzmann constant). It is usually presented as the Debye length $\lambda_{D}=\kappa^{-1}$ that describes the thickness of the double layer at which the electrostatic potential is decayed to $1 / \mathrm{e}$ of the surface potential. The reduction of the Debye length with increasing electrolyte addition is schematically shown in Figure 3.2.1d. 


\subsubsection{DLVO theory}

The DLVO theory combines the electrostatic interactions and van der Waals interactions to the total interaction energy between two like-charged colloidal particles at the distance $d$. The hardsphere repulsion potential (Born repulsion) that induces strong repulsions at distances smaller than the particle radius can be included as well and is usually modeled as $d^{-12}$ dependence.

$$
\begin{gathered}
U_{D L V O}(d)=U_{v d W}(d)+\Psi(d)+U_{B o r n}(d) \\
U_{D L V O}(d)=-\frac{A_{H} R}{12 d}+\Psi_{0} \cdot \exp (-\kappa d)+\frac{1}{d^{12}}
\end{gathered}
$$

a)

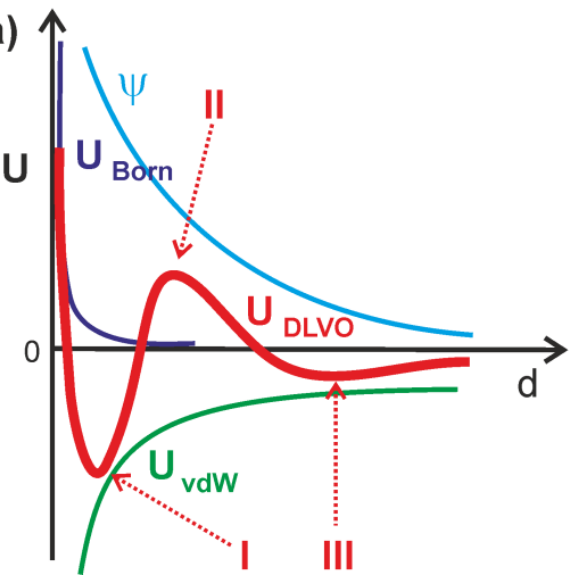

b)

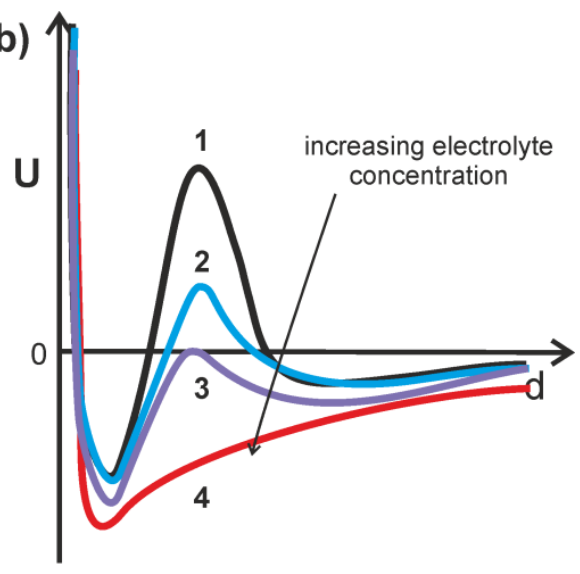

Figure 3.2.2. DLVO theorie: a) total interaction potential of two spheres according to the DLVO theory as a sum of the individual contributions. b) Effect of electrolyte addition.

Figure 3.2.2a shows the DLVO potential in dependence of the distance $d$ of one particle from the surface of a second. At small distances $(d<0)$, the Born repulsion prevents the particles from overlapping. A primary minimum at small, positive distances from the particle surface appears (I) that is caused by the strong van der Waals attractions at close distances. The electrostatic repulsion - if sufficiently high - induces a primary maximum at larger distances (II). Finally, a shallow secondary minimum is visible at even higher distances (III). In terms of colloidal stability, the primary minimum is referred to as coagulation. Once the particles reach this minimum, they are irreversibly agglomerated. This coagulation is prevented by the energy barrier arising from the electrostatic repulsion. The height of the energy barrier determines the stability of the dispersion. The second minimum is referred to as flocculation and is usually reversible. Figure 3.2.2b shows the effect of changing the electrolyte concentration. As the electrolytes increasingly screen the charges, they reduce the electrostatic stabilization. Thus, the 
colloidal dispersion is increasingly destabilized from a highly stable dispersion (1) to a slowly coagulating dispersion with a low energy barrier (2) to a completely unstabilized, rapidly coagulating dispersion (4). The critical coagulation salt concentration is shown in (3). The potential at the energy barrier is zero. For a stable electrostatically stabilized dispersion, the $\zeta$ potential is typically $\pm 20 \mathrm{mV}$ or higher in magnitude.

\subsubsection{Colloids at interfaces}

The placement of colloids at an interface introduces two additional forces that act on the particles by the very nature of the interface and thus, modify the total interaction potential. These two contributions are capillary forces that arise due to ability of the liquid interface to deform in the presence of particles and dipole interactions that originate from the difference in the dielectric constants between the two media. Here, a qualitative discussion of the two forces is given.

\subsubsection{Why particles are trapped at an interface}

An inherent property of colloidal particles is their ability to attach to interfaces as their presence at an interface forms an energy well that effectively traps the colloid at a fixed position between two materials. This behavior has been thermodynamically described by Pieranski ${ }^{[3]}$ in 1980 and has been exploited for the stabilization of emulsions by other colloids (Pickering emulsions) and for the crystallization of two-dimensional crystals (chapter 3.3.1.2). As the assembly of colloids at the air/water interface will be used in this thesis, the argumentation of Pieranski is briefly presented in the following.

The formation of an interface between two materials A and B cost an amount of interfacial energy $E_{A B}$ that is proportional to the area of the interface and the interface tension between the materials $\gamma_{A B}$. The presence of a colloidal particle at the interface changes the interfacial energy in dependence of the colloids' position with respect to the interface. 
a) air

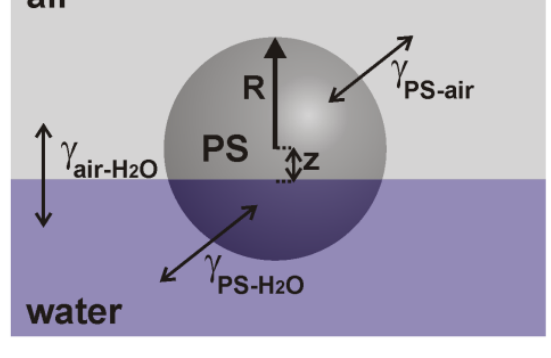

b)
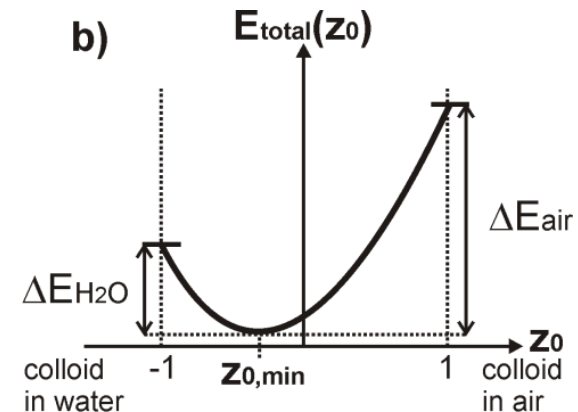

Figure 3.2.3. a) Geometrical situation of a polystyrene sphere at the air/water interface. $z$ denotes the deviation of the immersion depth from the symmetrical immersion with the particle's equator forming the three-point contact line. b) Energy well caused by the presence of the colloid at the interface.

Figure 3.2.3a shows a simplified model of a polystyrene colloidal particle at the air/water interface with the respective interfacial energies (interface bending due to capillary forces as described in chapter 3.2.2.3 is neglected). Using geometric arguments, the resulting total interfacial energy at a certain immersion depth of the particle can be calculated. $z$ denotes the deviation from symmetrical immersion (half-half immersion of the colloid with the equator forming the three-point contact line). In analogy, the normalized deviation can be defined as $z_{0}=z / R$ with the particle radius $R$. The three contributions to the total interface energy are:

$$
\begin{gathered}
E_{P S-a i r}=\gamma_{P S-a i r} \cdot 2 \pi R^{2}\left(1+z_{0}\right) \\
E_{P S-H 2 O}=\gamma_{P S-H 2 O} \cdot 2 \pi R^{2}\left(1-z_{0}\right) \\
E_{\text {air-H2O }}=-\gamma_{\text {air-H2O }} \cdot \pi R^{2}\left(1-z_{0}^{2}\right)
\end{gathered}
$$

The first equation describes the contribution of the sphere-air interface, the second line the contribution of the sphere-water interface and the last line represents the energy that is gained by the reduction of the air/water interface area by the colloid.

The total interface energy $E_{\text {total }}$, normalized to the size of the sphere, is thus:

$$
E_{\text {total }}=\frac{E_{P S-a i r}+E_{P S-H 2 O}+E_{\text {air }-H 2 O}}{\gamma_{\text {air }-H 2 O} \cdot \pi R^{2}}
$$

With the abbreviations $a=\frac{\gamma_{P S-\text { air }}}{\gamma_{\text {air }-\mathrm{H} 2 \mathrm{O}}}$ and $b=\frac{\gamma_{P S-\mathrm{water}}}{\gamma_{\text {air }-\mathrm{H}_{2} \mathrm{O}}}$, the total interface energy with respect to the colloid position can be written as:

$$
E_{\text {total }}\left(z_{0}\right)=z_{0}^{2}+2(a-b) z_{0}+2 a-2 b-1
$$


As depicted in Figure 3.2.3b, the equation has a minimum at $z_{0, \min }=b-a$ :

$$
E_{\text {total,min }}\left(z_{0}=(b-a)\right)=2 a+2 b-1-(a-b)^{2}
$$

The results can be generalized and demonstrate that colloids are trapped in an energy well at an interface between $\mathrm{A}$ and $\mathrm{B}$ as long as:

$$
-1<z_{0, \min }=b-a=\frac{\gamma_{\text {coll }-B}-\gamma_{\text {coll }-A}}{\gamma_{A-B}}<1
$$

In the equation, the gammas represent the interface tensions between the different materials; $z_{0}>1$ implies colloids completely in phase $\mathrm{A} ; z_{0}<-1$ correspond to colloids completely immersed in phase B.

For the polystyrene sphere at the air/water interface, Pieranski calculated the energy well using values for the different interfacial tensions tabulated by Sheppard $(a=0.49 ; b=0.14){ }^{[39]}$

$$
\begin{gathered}
z_{0, \min }=b-a=-0.35 \\
E_{\text {total,min }}\left(z_{0, \min }\right)=0.14 \\
E_{\text {Air }}(z=1)=4 a=1.96 \text { (sphere in air) } \\
E_{H 2 O}(z=-1)=4 b=0.64 \text { (sphere in water) }
\end{gathered}
$$

These values can further be used to calculate the energy needed for the re-entry of the colloid from its minimum position at the interface into either phase $\left(\Delta E_{\text {air }}\right.$ for the re-entry of the colloid into air and $\Delta E_{H 2 O}$ for the reentry into water). The absolute values shown in brackets are exemplarily calculated for a sphere with a radius of $100 \mathrm{~nm}$ :

$$
\begin{gathered}
\Delta E_{\text {air }}=E_{\text {air }}-E_{\text {total,min }}=1.82\left(4.2 \cdot 10^{-15} \mathrm{~J}=1.0 \cdot 10^{6} k_{B} T\right) \\
\Delta E_{\text {water }}=E_{H 20}-E_{\text {total,min }}=0.50\left(1.15 \cdot 10^{-15} \mathrm{~J}=2.6 \cdot 10^{5} k_{B} T\right)
\end{gathered}
$$

The results show that there is a minimum energy position for a polystyrene colloid at the interface and that the removal of the colloid from its minimum position at the interface into the water-phase requires energy of approximately one million $k_{B} T$. Thus, a particle in contact with the interface will not be able to leave into the subphase and is very efficiently trapped in the energy well. 


\subsubsection{Dipole interactions}

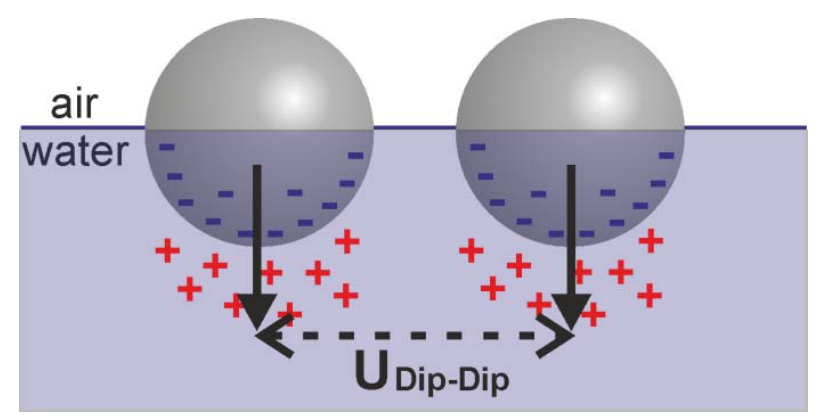

Figure 3.2.4. Two colloidal particles at the air/water interface. The asymmetry of the counter-ion clouds induces repulsive dipole moments normal to the interface.

In Figure 3.2.4, two electrostatically stabilized colloids at the air/water interface are shown. The distribution of charges, caused by the dissociation of surface functionalities (e.g. sulfonic- or acrylic acid groups) is asymmetric with respect to the interface. This is a consequence of the different dielectric constants of the two media. Water stabilizes ions very efficiently and favors dissociation of surface groups while non-polar media (air, oil) do not support dissociation. The resulting counter-ion cloud is asymmetric as well as sketched in Figure 3.2.4. Associated with this asymmetric charge distribution are dipole moments perpendicular to the surface. As the particles are trapped at the interface, the dipoles cannot align and thus induce a repulsive force in between the particles. Pieranski estimated the interaction potential by assuming a magnitude of the dipole moments given by the total number of charges per particle $z \cdot e$ divided by the Debye screening length in water $\kappa^{-1}$ :

$$
U_{d i p-\operatorname{dip}}(D)=\frac{\left(\frac{z \cdot e}{\kappa}\right)^{2}}{4 \pi \varepsilon \varepsilon_{0}} \cdot \frac{1}{D^{3}}
$$

In the expression, $\varepsilon_{0}$ is the vacuum permittivity and $\varepsilon$ the dielectric constant of water. The dipole dipole interaction is thus a long-range repulsion $\left(\sim D^{-3}\right)$ and contributes to ordering processes of colloids that form non-close-packed monolayers at interfaces (compare chapter 3.3.2.3). 


\subsubsection{Capillary forces}

Capillary forces occur when two or more particles are positioned at a fluid interface. They are very long range and attractive for identical colloids. The origin of capillary forces is a deformation of the liquid interface around the colloids caused by the finite contact angle of the particles. This deformation translates into a Laplace pressure that causes attraction of the colloids to minimize the bending of the interface.

Two distinct situations where capillary forces occur can be distinguished and are illustrated in Figure 3.2.5. For colloids at solid surfaces, the presence of a thin film of liquid with a height smaller than the diameter of the colloids induces immersion forces (a) while particles at the air/water interface experience flotation forces. Both types of capillary forces are of crucial importance for the assembly of colloids into ordered arrays as will be shown in the next chapter. It is noteworthy that capillary forces can also be repulsive if colloids with different contact angles are applied (i.e. hydrophilic and hydrophobic particles) as the curvature of the liquid film would increase upon closer proximity. ${ }^{[38]}$

a) Immersion force

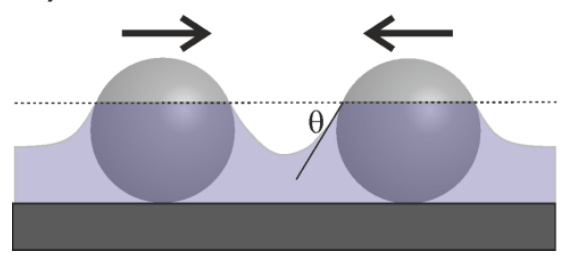

b) Flotation force

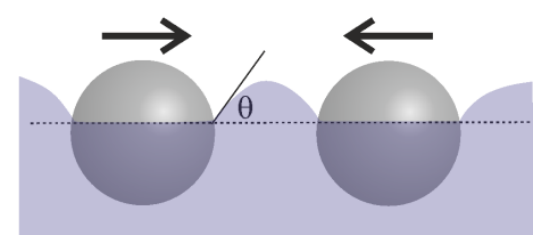

Figure 3.2.5. Capillary forces between two colloids caused by the deformation of the interface. a) Flotation force; b) immersion force.

The exact mathematical treatment of such forces is complex and has been elaborated in detail by $\mathrm{Chan}^{[40]}$ and Kralchevsky ${ }^{[41-42]}$ for flotation and immersion forces, respectively.

From the theoretical treatment, the following expressions for the two types of capillary forces are derived:

$$
\begin{aligned}
& F_{\text {flotation }} \sim \frac{D^{6}}{\gamma} K_{1}(q r) \\
& F_{\text {immersion }} \sim D^{2} K_{1}(q r)
\end{aligned}
$$

The different variables in the equation are: $D$ is the colloid diameter, $\gamma$ the interfacial tension and $K_{l}$ the Bessel function of first kind that depend on the radii of the three phase contact lines $r$ 
and the capillary length $q$ that is related to the density difference between the two media $\Delta \rho$ and the interface tension $\gamma\left(q^{2}=\frac{\Delta \rho \cdot g}{\sigma}\right)$.

The character of the capillary forces is a very long range attraction that extends to distances of several hundred nanometers. ${ }^{[38]}$ Thus, it governs crystallization processes in two dimensions as will be discussed in the next chapter.

\subsection{Two-dimensional colloidal crystallization}

At the very heart of this thesis lies the ability of monodisperse colloidal particles to form densely-packed, two dimensional crystals, commonly referred to as monolayers. The controlled formation of such monolayers has been attracting immense attention as they provide a simple, fast and cheap means to create diverse nanostructures in a defined fashion. Such nanostructures have been used in a plethora of applications, ranging from fundamental contributions to the field of plasmonics, the creation of antireflective and superhydrophobic surfaces, the controlled placement and the detection of biologically relevant molecules as well the creation of nanosized membranes (see chapter 3.4.4).

In the following chapters, a detailed overview of different crystallization techniques known in literature is given. First, methods leading to classical, close-packed monolayers are presented. Second, more recent developments in 2D - colloidal crystallization are reviewed that aim at increasing the versatility of accessible configurations. Two main research directions can be identified. The creation of non-close-packed monolayers has gained attention as such architectures significantly increase the flexibility of colloidal lithography to yield more complex nanostructure arrays. A focused research effort has also been initiated on binary colloidal monolayers, comprised of a densely packed monolayer of large particles with smaller colloids located in the interstitial sites. In contrast to crystals of uniformly sized particles, such binary architectures feature a rich variety of surface structures and symmetries depending on the size and number ratios of the large and small colloids applied.

\subsubsection{Close-packed colloidal monolayers}

This chapter provides an overview of existing monolayer crystallization techniques, compares different mechanisms and critically assesses possibilities and limitations of the different processes. 
A variety of different crystallization techniques exists in literature. ${ }^{[43-44]}$ From a conceptual point of view, all these techniques can be divided into two main categories: direct assembly on a solid substrate and liquid interface-mediated processes. Even though quality, possibilities, and limitations strongly differ between the fabrication techniques, there are certain key differences between the two main categories that shall be briefly discussed before going into detail. In general, interface-mediated processes possess several inherent benefits. i) By nature of the preassembly at an interface, the resulting crystal is necessarily two-dimensional. No precautions have to be taken to prevent the formation of multilayers. ii) By virtue of the same argument with the interface being liquid, the colloidal particles retain a lateral mobility that allows them to assemble into a minimum free energy position given that the energy barrier induced by electrostatic repulsion is sufficiently high. Hence, a high order in the resulting monolayer can be expected. iii) A key parameter that can be exploited to tune and control the crystallization process is the nature and composition of the subphase, not being available for direct assembly processes. iv) The choice of substrates is less critical with respect to material and surface properties for interface-mediated processes as the close-packed monolayer already assembles at the interface and only has to be transferred to the substrate of choice. In contrast, almost all direct assembly processes are very restricted in the choice of substrates and require certain properties (e.g. hydrophilicity, surfaces charges). v) In many cases, though exceptions exist, direct assembly methods are experimentally more straightforward as they do not require any special instruments. However, the price to be paid for simplicity is a very tight control over both experimental and ambient conditions that can be demanding to regulate and cumbersome to optimize.

A strong advantage of direct assembly methods is their higher flexibility in the preparation of different colloid arrangements with pre-structured substrates. This is a consequence of the crystallization process where the individual colloids can adapt to surface confinements of the substrate directly during crystallization. For interface-mediated processes, the adaption to nanoscale morphologies of the substrate is difficult as - in most cases - the monolayer is transferred to the substrate in its energetically most favorable, crystalline position and will not tend to disassemble into different arrangements.

A table (Table 3.3.1) that summarizes and compares the different preparation methods described in the following is presented at the end of this chapter. 


\subsubsection{Direct assembly methods}

The direct assembly methods can be further divided into two subcategories: evaporation induced processes and processes using external forces. While the latter are usually fast, the application of strong force fields can lead to irregularities in the order of the monolayer. In contrast, evaporation induced methods are inherently rather slow. If experimental conditions are tightly controlled (including ambient temperature and humidity), a highly ordered monolayer is produced. A severe limitation of evaporation induced processes is their sensitivity to the meniscus of the solvent front. Meniscus pinning due to imperfections of the substrate easily leads to ruptures in the resulting monolayer.

\subsection{Evaporation induced methods}

\section{Controlled horizontal evaporation (drop casting)}

The horizontal evaporation method can be assumed to be the easiest method to create colloidal monolayers. In the simplest case, a drop of the colloidal dispersion is placed on a substrate (typically glass or silicon wafer) and after evaporation, areas covered with a close-packed monolayer are obtained. ${ }^{[45-47]}$

Pioneered by the Nagayama group in the 1990s, this method has been extensively employed to study the fundamental mechanism of the crystallization process. A two stage mechanism was proposed. Initially, the colloidal particles are dragged to each other by capillary forces ${ }^{[41]}$ when the height of the liquid film is comparable to the diameter of the colloidal particles. Once a crystallization nucleus is formed, a convective flow of further particles sets in that is driven by the evaporation of water in the formed monolayer (Figure 3.3.1). ${ }^{[41,48-50]}$

The process is able to produce ordered monolayers - or monolayer patches - on a small scale ( $\mathrm{mm}^{2}$ dimensions), but the homogeneous coverage of larger areas is impeded by two inherent problems accompanying the evaporation process. Meniscus pinning causes sudden jumps of the receding water-evaporation front and leads to stripe patterns of monolayer domains (Figure 3.3.1c). ${ }^{[48,51]}$ Additionally, control of the boundaries of the water droplets is of crucial importance to prevent coffee-stain effects. ${ }^{[48]}$ This effect, coined after the typical appearance of a coffee stain, describes the directed flow of particles to the outer part of a droplet that leads to the formation of colloidal multilayers at the rims and depletion of particles in the middle of the droplet. (Figure 3.3.1d). Denkov et al. used a ring partially wetted by the dispersion to seal the 
liquid droplet to avoid crystallization at the outer rim and hence, circumvent the coffee stain effect. $^{[48]}$

Summarizing, the method is valuable for its simplicity and yields highly ordered monolayers on small scales.
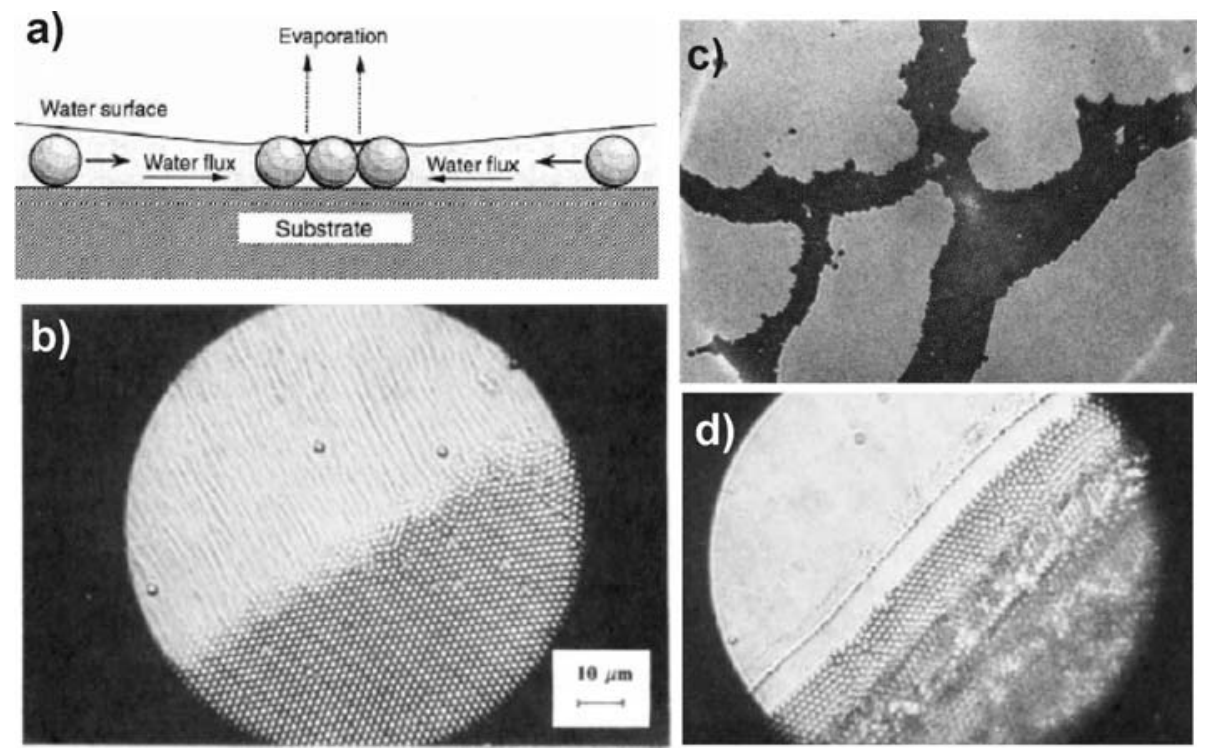

Figure 3.3.1. Controlled horizontal evaporation: a,b) mechanism of the monolayer formation: a) schematic illustration; b) optical microscopy image showing the flux of particles towards the crystalline monolayer. Both images are taken from Denkov et al. ${ }^{[49]} \mathrm{c}, \mathrm{d}$ ) Typical problems arising with this technique as shown by Denkov ${ }^{[48]}$ : c) submonolayer formation, d) coffee stain effect that leads to the formation of multilayers at the drying front.

\section{Vertical and horizontal deposition (convective assembly)}

A significant advance towards larger areas covered with a homogeneous colloidal monolayer was introduced by Nagayama 1996 with the vertical deposition process. ${ }^{[52]}$ They inserted a substrate into a colloidal dispersion and withdrew it vertically with a constant (slow) speed. Eventually, the colloids nucleate at the drying front to form a crystalline structure. When the substrate is slowly moved in opposite direction to the crystal growth with the right speed, a monolayer forms and subsequently growths as the substrate is continuously removed. The forces that govern the process are attractive capillary forced that drive the particles towards the crystals once the height of the water film drops below the colloid diameter. Additionally, more particles are delivered towards the crystal growth front by a water flux that is build up by the evaporation of water in the monolayer (Figure 3.3.2). The process is able to produce monolayers ${ }^{[22-53]}$ as well as ordered three-dimensional crystals. ${ }^{[54]}$ The major drawback of the process is the large number of process parameters that need to be controlled in order to yield 
continuous monolayers. These include the withdrawal speed of the substrate and its wettability as well as pressure, temperature and humidity. Additionally, as the particle concentration of the dispersion changes in the course of the process, accurate coverage of arbitrary large areas is impeded. ${ }^{[55]}$

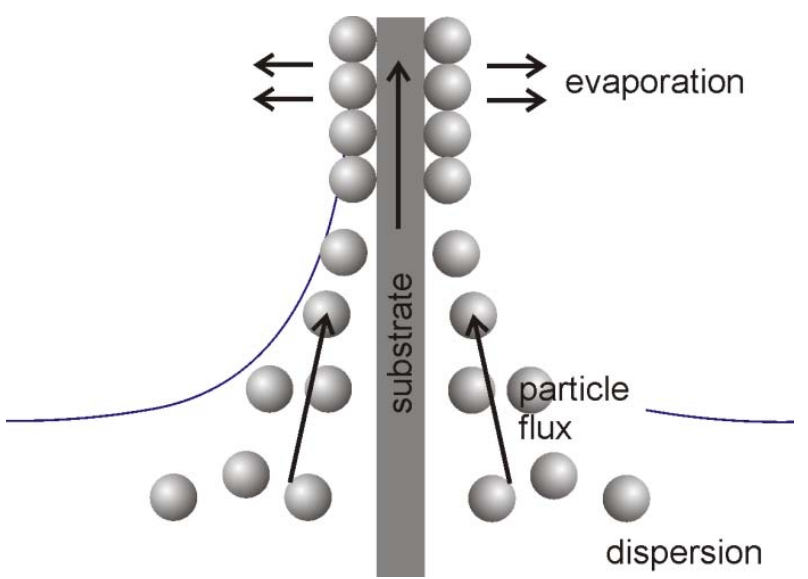

Figure 3.3.2. Schematic illustration of the controlled vertical deposition process. The substrate is lifted vertically. Once the height of the water layer becomes smaller than the size of the colloids, the crystallization process is initiated. Subsequently, a flux of particles towards the growing crystal growth sets in. The figure was adapted from Dimitrov. ${ }^{[52]}$

The process can also be conducted horizontally: The substrate is moved horizontally while a fixed confinement slide creates a permanently receding contact line. If the contact angle is low enough (i.e. below $20^{\circ[56]}$ ), a convective assembly of particles sets in following a similar mechanism as described for the vertical deposition. A higher contact angle does not allow for the creation of monolayers on flat substrates as the height of the water level will not become small enough to allow for capillary forces to act effectively. ${ }^{[56]}$ A microscope can be mounted on top of the set-up to observe the crystallization process directly. The temperature of the substrate is used to control the solvent temperature. Thus, a means to tune the evaporation rates and is at hand. If not all parameters are properly controlled multilayer or separated monolayer domains formation prevails. ${ }^{[57]}$

\subsection{External force induced methods}

\section{Spin coating}

Spin coating, established as the method for casting thin polymer films onto solid substrates, has been employed for colloidal crystal fabrication as well. The benefits of using spin coating for colloidal crystallization are at hand: Large areas can be covered in minutes. However, the 
formation of long range order in a crystal is difficult to achieve with high lateral shear forces and fast solvent evaporation characteristic for such processes.

Pioneered by the van Duyne group, ${ }^{[5-6]}$ several approaches and modification have been made. ${ }^{[58-}$ 60]

While first reports simply use spin coating as a means to get small domains of monolayers to be applied to lithographic processes, later contributions focused on optimizing the volatility of the dispersing media. Jiang et al. used silica particles dispersed in highly viscous triacrylate monomers paired with sophisticated gradual rotation protocols to produce three-dimensional crystals $^{[58]}$ and non-close-packed monolayers ${ }^{[59]}$ with satisfying order and optical properties. Due to the high viscosity, the particles gain longer time to crystallize. A photo-polymerization step is performed to add mechanical stability to the crystals. The organic nature of the solvent prevents the use of latex colloids in the same process. Additionally, the high viscosity and lower dielectric constant of the monomers might be demanding for proper colloidal dispersion and stability.

The creation of close-packed colloidal monolayers and crystal of polystyrene and silica particles was reported by Mihi et al. that changed the protocol to volatile solvent mixtures (water/ethanol/ethylene glycol) with higher vapor pressures and extremely low rotations speeds $(\sim 200 \mathrm{rpm})$ to allow for a proper ordering. ${ }^{[60]}$

\section{Electrostatic deposition}

Electrostatic deposition techniques exploit the attractive interaction between opposing charges by creating charged surfaces to attract oppositely charged colloidal particles.

Several conceptual approaches have been demonstrated. Hammond et al. used polyelectrolyte layers to produce patterned arrays of colloidal silica or polystyrene layers ${ }^{[61]}$ while Jonas et al. applied patterned, charged silane monolayers ${ }^{[62]}$ for the same purpose. The strong interaction that drives the assembly process unfortunately prevents a high order in the colloidal monolayer as the individual particles lack mobility to find their hexagonal environment. Instead, they stick to the surface and form rather disordered arrangements. A significant improvement was introduced recently by the Yang group who separated the crystal formation from the electrostatic attraction. ${ }^{[63]}$ They assembled a 3D crystal of negatively charged polystyrene particles in ethanol on a polyelectrolyte layer. The assembly in ethanol avoids the ionization of the polyelectrolyte so that a high order of the crystal is achieved. Subsequently the solvent is changed to water, leading to ionization of the polyelectrolyte layer. The top layers of the colloidal spheres are washed away in the water phase, whereas well-ordered colloids that are in contact with substrate tightly adhere due to the attractive electrostatic interactions, leading to the formation of ordered arrays of negatively charged 2D colloidal spheres (Figure 3.3.3). All of the 
mentioned processes are able to produce defined patterns of monolayers as well by prestructuring of the substrate. ${ }^{[61-63]}$
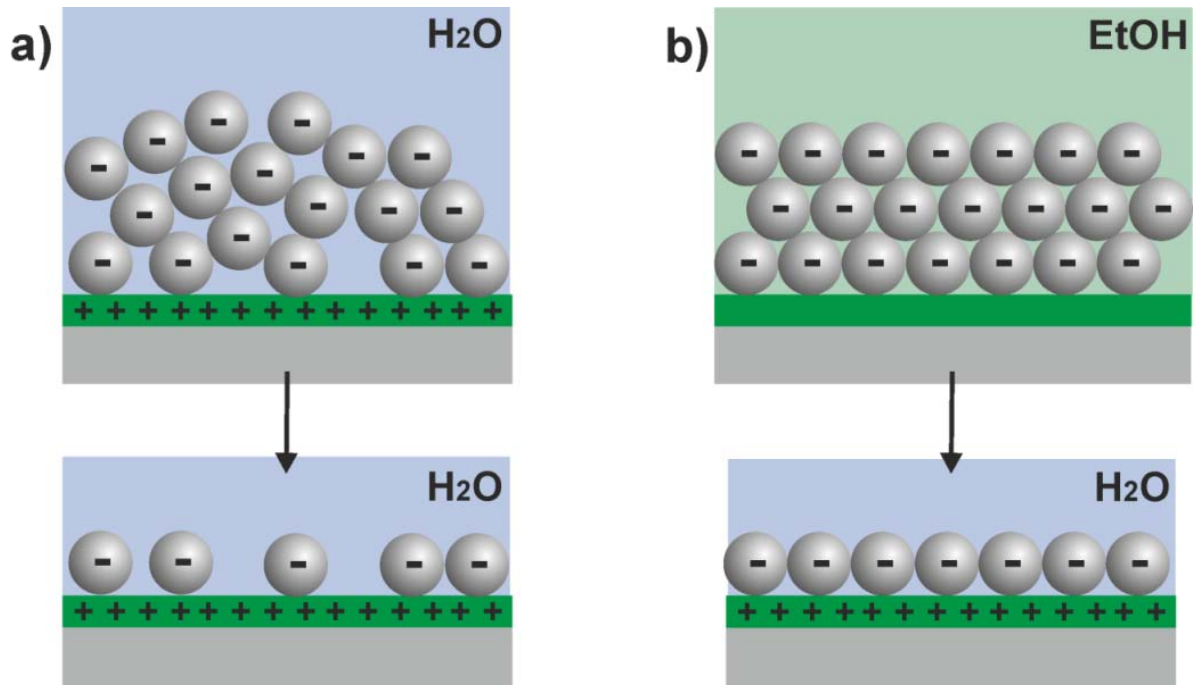

Figure 3.3.3. Electrostatic deposition process as demonstrated by Zhang et al. ${ }^{[63]}$. a) Assembly process of a three dimensional crystal in water (charged polyelectrolyte) resulting in poor ordering of the monolayer. b) Assembly process in ethanol (non-charged electrolyte). The absence of charges induces a higher order in the monolayer assembly. Changing from ethanol to water induces mediates an electrostatic adhesion of the first layer that is not removed by rinsing.

\section{Electrophoretic deposition}

Closely related to electrostatic deposition processes is the technique of electrophoretic deposition. Both take advantage of charge attraction. However, while the presence of charges is static and induced by the substrate in the former process, the latter uses conducting substrates as electrodes to induce electrostatic assembly. Similar to the electrostatic deposition process, the strong electrostatic attraction between the colloids and the substrates renders high ordering in the monolayer difficult. However, reports on pre-structured substrates demonstrate high order of the monolayers if the width of electroconductive grooves were commensurate with a discrete number of colloid spheres. ${ }^{[64]}$

In a different approach, Dziomkina et al. used pre-structured electrodes to produce highly ordered colloidal monolayers and crystals with symmetries not achievable by natural crystallization processes (i.e. quadratic arrangements). ${ }^{[65]}$

The approach was also extended to planar monolayers crystallized by alternating electric fields at the electrodes ${ }^{[66]}$ and in a field gradient between two electrodes. ${ }^{[67]}$ It was found that the application of alternating electric fields produced monolayers with a higher degree of long range 
order compared to constant electric field as nucleation rates can be controlled more precisely with alternating fields. ${ }^{[6]}$

\section{Sedimentation}

The sedimentation of colloids has been employed for the fabrication of 3D silica colloidal crystals. ${ }^{[68-69]}$ Park et al. also reported the process to be able to produce close-packed monolayers if the crystallization volume is restricted in a cell and parameters are properly controlled. ${ }^{[70]}$

\subsubsection{Liquid interface-mediated methods}

The second main category for crystallization processes are liquid interface-mediated methods were colloidal crystallization takes place at a liquid interface. Several inherent advantages of the liquid nature of the interface have been accounted for in the beginning of this chapter.

In contrast to direct assembly methods, where, in most cases, convective liquid flows and immersion forces are the key players that drive the assembly process, interface mediated methods are characterized by a longer crystallization timescale: The colloids spread onto a liquid film possess a high degree of lateral mobility. Three forces are predominant for the assembly process: Capillary forces (flotation force) cause long range attractions, van der Waals forces add to shorter range attraction and both are partially counterbalanced by repulsive electrostatic and dipole forces. The balance of these forces is crucial for the assembly of high quality monolayers: Especially lacking electrostatic repulsion leads to disordered monolayers as the individual particles are attracted by capillary forces and "freeze" upon contact by van der Waals attraction. An increase in electrostatic repulsion induces a higher contact energy barrier that allows for a higher mobility of the particles that can consequently find their minimum free energy position. The subphase in liquid interface-mediated methods is hence a key player for the balance of forces as especially the electrostatic force can be tuned, e.g. by $\mathrm{pH}$ or addition of electrolytes.

The balance of forces seems to be critically different when changing from air/water to oil-water interfaces. While close-packed monolayers typically result in the former case, the appearance of non-close-packed structures prevails at oil-water interfaces. For air/water interfaces, attractive capillary forces, apparently dominate, as can be seen from the appearance of brightly colored monolayer patches upon spreading ${ }^{[71-73]}$ that indicate the formation of densely packed islands. At the oil-water interface on the contrary, repulsive forces seems to be significantly stronger as no 
direct contact between colloids takes place and the colloids uniformly distribute throughout the interface. Furthermore, it is reported that the interparticle distance can be tuned as a function of the number of colloids added; thus corroborating with the model of repulsive particles trying to avoid each other to the maximum extend. ${ }^{[71,74]}$ Figure 3.3.4 illustrates the effect of the interface composition of the resulting colloid assembly. The difference between the two cases is attributed to two factors. i) The position of a colloid at the interface (i.e. its contact angle) strongly differs between air/water and oil-water interfaces. At the air/water interface, colloids typically possess low contact angles, i.e. the colloid is immersed in the water phase to a high extent (see chapter 5.2.3.2.5). ${ }^{[73]}$ In contrast, at an oil-water interface, the colloids' equator is shifted more towards the oil phase. ${ }^{[71]}$ This affects the strength of the repulsive dipole interactions arising from the asymmetric counter-ion cloud (chapter 3.2.2.2), the magnitude of electrostatic repulsive forces and capillary forces. ii) It has been postulated that a small amount of surface charges might be stabilized in the hydrophobic area by traces of water remaining at the particles surface (e.g. due to surface corrugations). ${ }^{[71,75]}$ These charges induce a strong electrostatic repulsion as no ions are present in the oil phase to screen them. Hence, in case of the oil-water interface, the higher surface area of the colloids lead to more unscreened charges and induce an overall repulsive character. ${ }^{[71]}$ First, only assembly methods leading to closepacked architectures are presented. In the next chapter (3.3.2), non-close-packed architectures prepared from assembly and transfer at the oil-water interface will be reviewed.
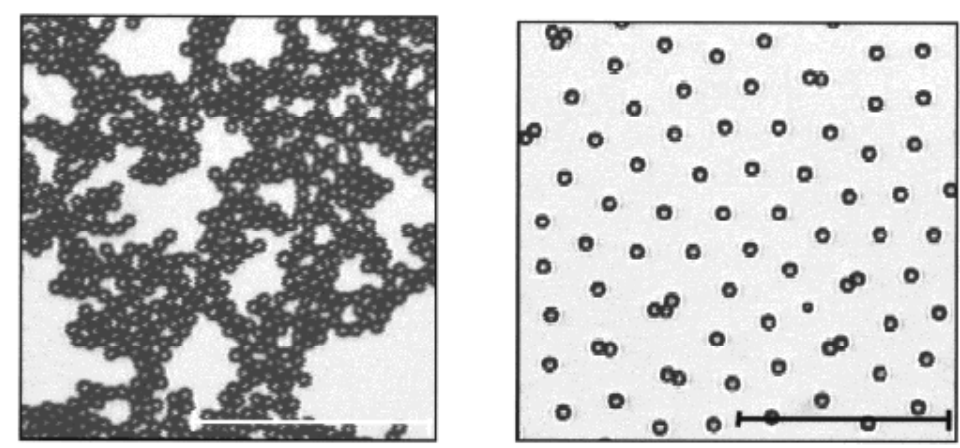

Figure 3.3.4. Difference of the colloid assembly at the air/water interface (left side) and oil-water interface (right side). The image is taken from Aveyard et al. ${ }^{[71]}$

The outstanding method among interface-mediated methods is the use of Langmuir troughs that can be considered as the predominant method in two-dimensional crystallization. However, several novel methods using the air/water interface for assembly as well but without requiring special equipment have been reported in recent years.

The first report to mention the crystallization of mesoscale colloids at an interfaces dates back to 1980 when Pawel Pieranski - in the pursuit of finding a suitable experimental system to study 
two-dimensional phase transitions - discovered that polystyrene particles are essentially trapped at the air/water interface by an energy well ${ }^{[3]}$ (chapter 3.2.2.1) A similar behavior for macroscopic colloids was experimentally realized by Sheppard and Tcheurekdjian as early as $1968 .^{[39,76]}$

\section{Crystallization on a Langmuir trough}

The use of Langmuir troughs to study colloidal crystallization dates back to experiments from Sheppard and Tcheurekdjian with macroscopic particles at the air water interface. ${ }^{[39,76]}$ and was taken up by Fulda and Tieke in the 1990s for the examination of nanoscopic polystyrene colloids. ${ }^{[77-78]}$ The surface-pressure area isotherms of colloid films are extremely steep as is expected in a incompressible, crystalline and stiff film at the interface. ${ }^{[77-79]}$ Transfer of the monolayer is typically performed in this steep regime before the collapse.

Since this early work, the focus has shifted away from fundamental studies towards the exploitation of Langmuir techniques for the preparation of high quality mono- and multilayers. A recent review of Bardosova et al. reports on progress for the $3 \mathrm{D}$ crystal assemblies with an emphasis on their application as photonic crystals. ${ }^{[80]}$

Colloidal monolayers consisting of micrometer-sized polystyrene particles were first deposited on solid substrates by Lenzmann et al. ${ }^{[81]}$ The colloids were spread from ethanolic dispersions and transferred manually by immersion of a glass slide and slow withdrawal under a shallow angle. The resulting monolayer showed a high order. The first report on a classical LangmuirBlodgett transfer of mesoscale silica particles came from van Duffel and coworkers in 2001 who fabricated monolayers by spreading $\mathrm{SiO}_{2}$ nanoparticles from ethanol/chloroform mixtures. The resulting monolayers were of low ordering. ${ }^{[82]}$ Similar results were reported by several other groups for silica particles deposited via the Langmuir-Blodgett technique. ${ }^{[83-85]} \mathrm{A}$ significant improvement in quality was reported for silica particles with surface methylation. ${ }^{\text {[9] }}$ Very well ordered silica monolayers were reported from Reculusa et al. using allyl-modified silica particles and optimized protocols. ${ }^{[86]}$ To increase the order in monolayers, the addition of various surfactants was proposed ${ }^{[82,87]}$ but seem redundant from today's perspective as another innovation paved the way for true high quality monolayers: Weekes et al. modified the way of colloid addition by using a glass slide with an angle of approximately $45^{\circ}$ with respect to the water surface and allowed the dispersion to gently slide onto the trough via the glass. ${ }^{[72]}$ Thus, colloidal monolayers with excellent quality were produced. The exact reasons for the high ordering have not been completely resolved. However, it can be argued that the slow and gentle immersion via the glass slide induces less disturbances of the water interface compared to the direct spreading and hence, facilitates the crystallization process. Recently, the same group was 
able to further increase the quality into macroscopic single crystal domains by tuning the electrostatic repulsion forces by the $\mathrm{pH}$ of the subphase. ${ }^{[8]}$

\section{“Monolayers to go"}

Several innovative concepts have been introduced, which also use an air/water interface for colloidal assembly, but circumvent the rather time-consuming compression step on the Langmuir trough. The aim of these methods is to produce highly ordered monolayers without special equipment or tight control of experimental conditions in a simple and fast process that can be performed in a "cannot mess it up" fashion with the ultimate goal to make colloidal monolayers available wherever needed even to untrained persons.

In 2009, Retsch et al. proposed a concept coined "floating monolayers" ${ }^{[55]}$ In this two-step procedure, a glass slide is covered with sparsely distributed colloids prepared by a simple spincoating step. This slide is subsequently immersed into water containing a small amount of sodium dodecyl sulfate (SDS) molecules. Upon immersion, the colloids assemble at the threephase contact line (glass/air/water) into a densely packed monolayer that remains afloat on the water surface once the glass slide is completely immersed. The monolayer can then be manually transferred to arbitrary substrates.

A process termed vortical deposition was introduced by Pan and coworkers. ${ }^{[89]}$ They used a dynamic (vortical) interface for the assembly, created by a magnetically stirred water surface and confined by a Teflon ring. To this surface, small drops of polystyrene particles dispersed in ethanol are added. In a way similar to spreading on a Langmuir trough, the particles remain at the air/water interface and form close-packed structures. The rotation introduces an additional centrifugal force that drives the particles towards the vortex center and facilitates a dense packing. Once the interface is saturated, the addition is stopped and the stirrer switched off. The interface relaxes and a colloidal monolayer with a surprisingly high quality is formed. Transfer is readily achieved by immersion of a hydrophilic substrate followed by careful withdrawal.

\subsubsection{Summary of the crystallization methods}

As has been shown in the previous chapters, a vast variety of crystallization methods for the preparation of close-packed colloidal monolayers exist. Table 3.3.1 gives an overview of the different methods reported in literature and briefly lists advantages, disadvantages as well as the corresponding references. 
Table 3.3.1. Overview over crystallization methods for close-packed colloidal monolayers

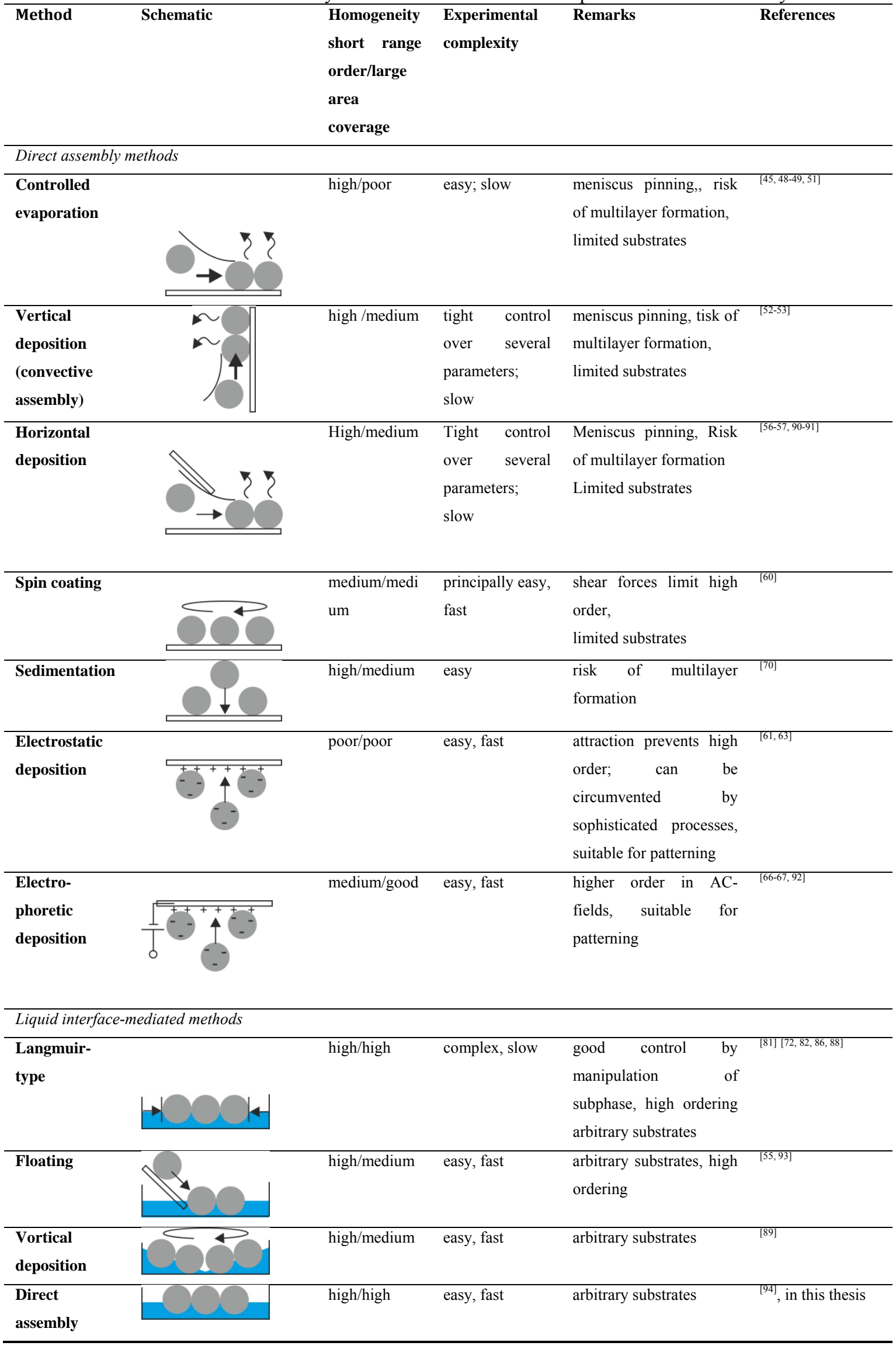




\subsubsection{Non-close-packed monolayers}

Non-close-packed monolayers find increasing applications for the construction of highly symmetric arrays of complex nanostructures as the individual colloids are sufficiently separated in space to allow for more sophisticated lithography processes (including e.g. angular evaporation and etching steps) compared to the classical nanosphere lithography with closepacked particle layers.

Three approaches can be distinguished. First, close-packed monolayers can be crystallized and further processed into non-close-packed structures, either using elastomeric substrates to spatially separate the particles by mechanical means or by applying a plasma-induced size reduction step. Second, using polymeric additives in a spin coating process, non-close-packed architectures can be yielded by selective removal of these additives. Finally, using an assembly process at the oil-water interface, a direct crystallization of colloidal particles into non-closepacked architectures can be realized.

\subsubsection{Non-close-packed architectures from close-packed monolayers}

This method can be considered the most straightforward approach to non-close packed architectures as the full set of crystallization possibilities for close-packed architectures can be exploited to tailor the crystallinity of the monolayer. In a second step, the individual colloids need to be separated in a symmetric fashion: The contact position of the colloids with the substrate must not be affected by the process if highly ordered non-close-packed architectures are desired. Hence, energy has to be distributed isotropically to overcome the mutual attraction of the colloids. Two processes have been established for this transformation. i) Plasma treatment of the monolayers can be used to reduce the size of the individual colloids and hence, produce a non-close-packed arrangement (Figure 3.3.5a). ${ }^{[95]}$ ii) An elastomeric substrate can be applied for the crystallization process (coined deformable soft lithography) (Figure 3.3.5b) ${ }^{[96-97]}$ Elongation of the substrate thus increases the distance between the individual particles. After transfer to a desired substrate using a polymeric adhesion layer, the non-close-packed architecture is fixed. Multiple elongation protocols also enable the construction sophisticated monolayer crystal structures such as square arrangements. ${ }^{[97]}$ 
a)
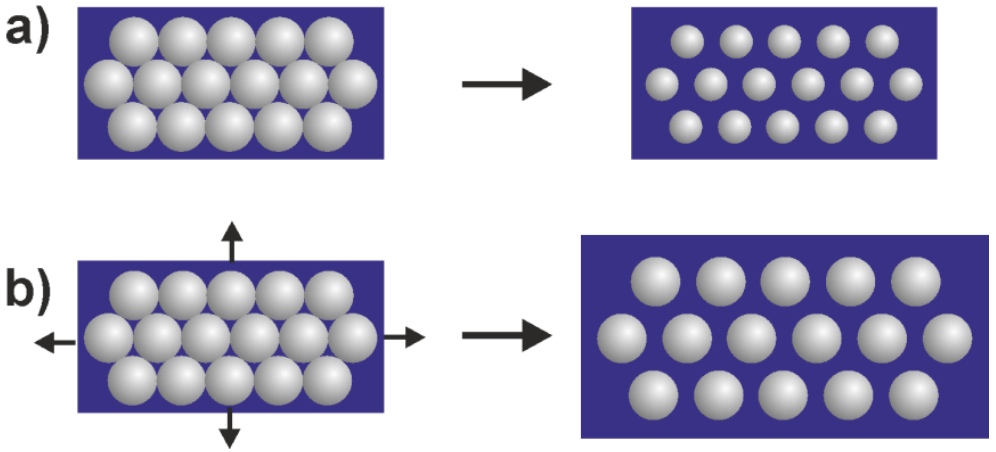

Figure 3.3.5. Fabrication of non-close-packed colloidal monolayers from close-packed arrangements. a) Plasma-assisted size reduction of the colloids. b) Stretching of an elastomeric substrate.

From a conceptual point of view, the two processes are diametric to each other. In the former process, the lattice spacing is retained while the colloid diameter is altered; the latter process induces changes in lattice spacing under conservation of the individual particle sizes. Beside their experimental simplicity, the advantages of such processes are the ease of adjustment of interparticle spacing; according to a desired application, one might chose to change lattice or particle diameter. Both plasma etching time or substrate elongation can subsequently applied and precisely adjusted to yield the desired spacing. The drawbacks are distinct for the two processes. The elastomer-method requires equipment with a high control over substrate elongation and the necessity of a transfer to a different substrate performed under stress. The plasma treatment step however, can compromise the quality of the individual particles due to surface corrugations and deviations from spherical shape in the course of the process.

\subsubsection{Non-close-packed monolayers by spin coating}

A spin coating process leading to non-close-packed silica monolayer architectures was reported by Jiang and McFarland in 2006. ${ }^{[59]}$ To overcome the difficulties of using the strong lateral forces of spin coating, they chose dispersions of silica particles with high solid content $(\sim 20$ wt-.\%) in a non-volatile, viscous triacrylate monomer paired with a substrate pretreatment (assembly of 3-acryloxy-propyl-trichlorosilane). A sophisticated spin coating protocol and subsequent photopolymerization of the monomer matrix resulted in the fixation of the silica spheres in an ordered hexagonal arrangement of non-close-packed colloids embedded in a cross-linked polymer matrix with wafer-scale dimensions. Selective combustion of the organic material gave rise to pure non-close-packed monolayers of silica spheres. In an inverse process, they also reported the selective removal of silica to produce nanoscale hole arrays that were 
subsequently filled with titania colloidal particles. ${ }^{[98]}$ A precise control over the interparticle distance using this process was not reported.

\subsubsection{Direct assembly of non-close-packed monolayers at the oil-water interface}

The self-assembly of latex particles at the oil-water interface is a direct method to yield nonclose-packed monolayers. As discussed in chapter 3.3.1.2, the nature of the colloids trapped at the interface is repulsive due to stronger dipole repulsion, reduced capillary forces and possible unscreened charges at the particle surface in the oil phase. ${ }^{[71,75]}$

The first report of interfacial, non-close-packed assemblies was based on the compression on a Langmuir trough by Aveyard et al. in 2000. ${ }^{[71]}$ They carefully investigated the behavior of sulfonated polystyrene particles at the octane-water interface, gave a model for the increased repulsion and reported a distortion of the non-close-packed monolayer upon high compression. However, they did not report a transfer of the resulting crystals to solid substrates. A similar behavior was also reported for silica particles at the octane-water interface. ${ }^{[99]}$ The interface position of the colloids, controllable by surface modification of silica spheres, is of crucial importance for the order in the non-close-packed systems as discussed above. High contact angles, i.e. a high volume fraction in the oil face leads to strong repulsions and a high order in the monolayer. ${ }^{[100]}$

The special properties of oil-water interfaces to yield highly regular non-close-packed monolayers was finally used by Isa and coworkers ${ }^{[74]}$ to create non-close-packed monolayers on solid surfaces. They assembled positively charged amidine-functionalized polystyrene colloids at the hexane-water interface and transferred them to a negatively charged substrate by vertical lifting (Figure 3.3.6).

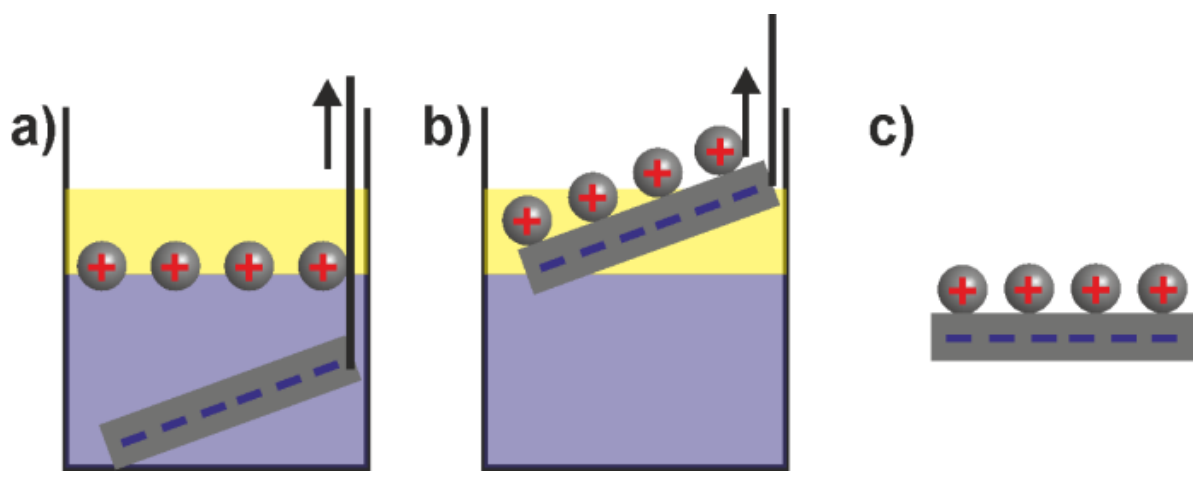

Figure 3.3.6. Transfer of non-close-packed monolayers from the hexane-water interface to solid substrates as proposed by Isa et al. ${ }^{[74]}$ a) Assembly of colloids at the interface. b) Vertical removal of immersed 
substrate with a slight angle. c) Resulting non-close-packed monolayer on the solid substrate.

The difficulty of the transfer procedure is to prevent the loss of position of the particles by viscous drags and capillary forces acting during solvent evaporation. ${ }^{[101]}$ Therefore, they chose attractive particle-substrate interactions. Hexane proved to be the solvent of choice for the oilphase as it possessed a low viscosity paired with a high volatility to overcome the forces during substrate drying.

\subsubsection{Binary colloidal monolayers}

Binary colloidal monolayers are two-dimensional crystals consisting of two different types of colloidal particles. Typically, large (L) and small (S) colloids are used to produce a densely packed monolayer of large colloids with a distinct number of small colloids located in the interstitial sites of the monolayer. Such configurations are typically denoted $\mathrm{LS}_{\mathrm{n}}$ structures to indicate the composition of the binary system. Compared to single-sized particle monolayers, binary arrangements thus possess a rich variety of different configurations that render them attractive for both fundamental research as well as applications for the creation of more complex surface patterns. Figure 3.3.7 shows some examples of possible crystal structures. Conceptually, one needs to distinguish between binary monolayers with a stoichiometric crystal structure as described above and patterned monolayers that consist of differently sized colloids as well but are comprised of individual domains of single-particle monolayers. ${ }^{[102-103]}$

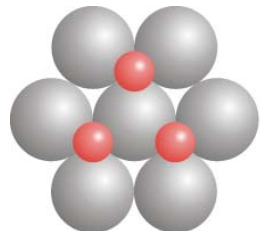

$\mathrm{LS}_{1}$

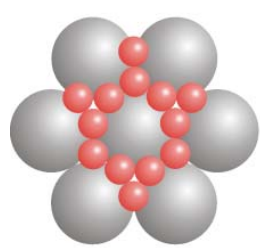

$\mathrm{LS}_{4}$

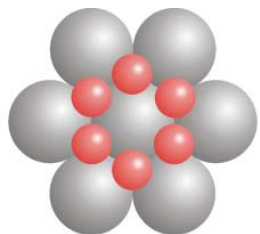

$\mathrm{LS}_{2}$

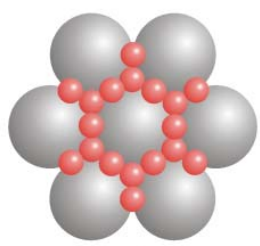

$\mathrm{LS}_{5}$

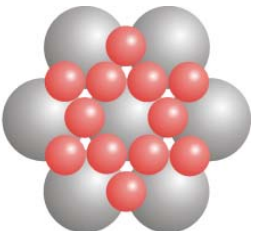

$\mathrm{LS}_{3}$

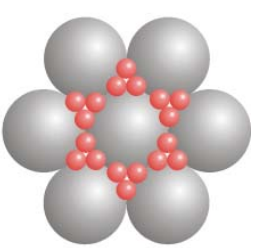

$\mathrm{LS}_{6}$

Figure 3.3.7. Examples of possible crystal structures in binary colloidal monolayers.

Even though identical techniques can be used, the crystallization of binary monolayers is substantially more demanding than the crystallization of single-sized particle monolayers. The 
presence of the small particles adds several process parameters that have to be additionally controlled. Up to now, a precise control over both long range order of the binary crystal and defined adjustment of interstitial site occupation remains challenging.

The assembly processes for binary 2D crystals can be divided into two major categories: sequential assembly (two-step processes) and co-assembly (one-step processes).

\subsubsection{Sequential assembly of binary monolayers}

The creation of binary colloidal monolayers was pioneered by the group of von Blaaderen ${ }^{[104]}$ who realized that close-packed monolayer of large spheres could be used as substrate in a controlled evaporation process to deposit small spheres on top of the monolayer to form binary crystals. During the evaporation process, the interstitial sites of the template monolayer act as traps for the small spheres that subsequently assemble into a regular superlattice on the largesphere monolayer with a high degree of order. For their experiments, van Blaaderen et al. restrained themselves to size ratios $\left(\gamma=D_{S} / D_{L}\right)$ close to 0.5 . The formed crystal structures depended on the concentration of small particles. Accordingly, LS, $\mathrm{LS}_{2}$ and $\mathrm{LS}_{3}$ configurations were found. The main limitation of the process is the change of concentration of particles during the drying process that inherently leads to changes in the monolayer configuration when covering larger areas on the substrate (Figure 3.3.8).

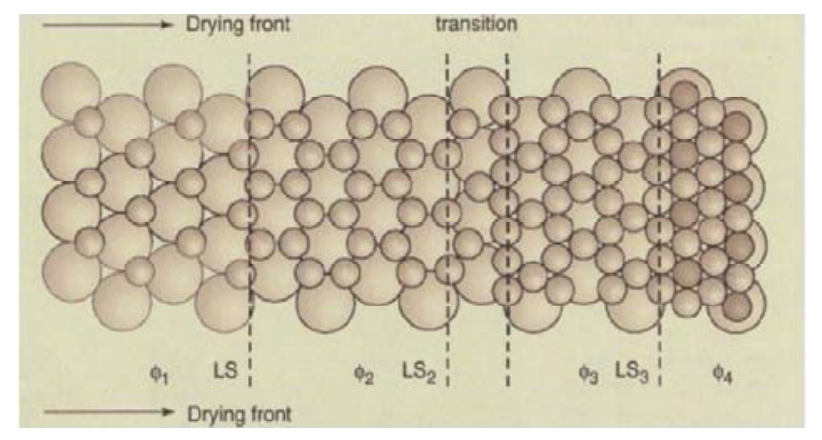

Figure 3.3.8. Binary colloidal monolayers by a sequential convective assembly of small particles in the interstitial sites of a close-packed monolayer of large particles. Scheme taken from Velikov et al. ${ }^{[104]}$

Depending on the concentration of the dispersion of small spheres, different configuration can be achieved.

In analogy to the developments in the crystallization processes for single-sized particle monolayers, the process of monolayer templating was further developed with vertical deposition techniques. Kim et al. ${ }^{[105]}$ coined the process confined convective assembly and reported on the crystallization of polystyrene particles with $\mathrm{LS}_{2}, \mathrm{LS}_{3}, \mathrm{LS}_{4}$ and $\mathrm{LS}_{5}$ configuration using size 
ratios between 0.30 and 0.65 . Further reports included combinations of small silica and large polystyrene spheres that lead to non-close-packed structures upon calcination. ${ }^{[106]}$ Even though the total order of the binary crystals seems high, it is necessary to keep in mind that such nonequilibrium processes are difficult to control over large areas as especially meniscus pinning effects lead to non-uniform coating.

Electrophoretic assembly using electrode substrates with a templating close-packed monolayer has been used to fabricate binary monolayers consisting of like-charge particles over an extremely wide range of size ratios $(0.1<\gamma<0.9)$ with a fair total order (Figure 3.3.9). ${ }^{[107]}$ The same process was a successfully applied even for the construction of ternary monolayers consisting of three differently-sized colloids. While the range of accessible size ratios is impressive, the control over the stoichiometry (i.e. the number of small particles in the interstitial sites) seems more difficult.

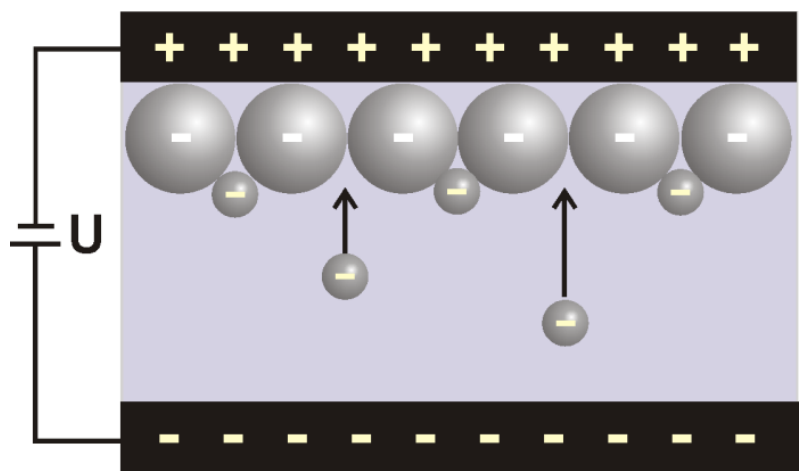

Figure 3.3.9. Electrophoretic assembly of like-charged colloids into binary monolayers according to Huang. ${ }^{[107]}$

The opposite approach, the assembly of opposite charged particles by immersion of a monolayer of negatively charged polystyrene particles into dispersions of positively charged particles was reported as well. ${ }^{[108]}$ However, due to the high attractive forces the total order of the monolayer is significantly lower compared to the other methods reported. The order could be controlled to some extend by the addition of electrolytes to reduce the mutual attraction. No control over the stoichiometry between the small and large spheres was reported. ${ }^{[108]}$

Spin-coating was also employed for the sequential fabrication of binary monolayers. ${ }^{[109]}$ Wang et al. employed a variety of size ratios $(0.25-0.60)$ for their experiments but were not able to precisely control the configuration of the binary system. The total order in the experiments reported was rather low as a consequence of the high lateral forces employed during spin coating. Thus, the method is well-suited for rapid fabrication processes but severely lacks precision in the resulting arrangements. 


\subsubsection{Co-assembly of binary monolayers}

The second approach towards binary colloidal monolayer is the direct co-assembly from binary mixtures. Several approaches based on crystallization techniques established for single-particle monolayers have been presented.

The idea of co-assembly was introduced by Kitaev and Ozin in 2003. ${ }^{[110]}$ They chose a rapid vertical evaporation process for the deposition to exclude undesired effects of gravity on the large particles. They were able to produce a rich variety of monolayer architectures by systematically varying the concentration of the small particles and the size ratio. Using size ratios below 0.225 , no ordered $2 \mathrm{D}$ crystals were yielded. In general, a large number of small particles surrounded the large spheres in an ordered fashion. The smallest amount of fillings of the interstitial sites was reported to be either triplet or tetrahedron like structures of small colloids. An exact analysis of the stoichiometry was not performed. A major drawback of this approach is the change of the concentration of small particles that will lead to a variation of the crystal structures obtained in the course of the evaporation process. ${ }^{[11]}$ Similar experiments conducted for the co-crystallization of gold rods and spheres were reported from the group of Luis Liz-Marzan who reported symmetric co-crystallization patterns. ${ }^{[12]}$

Kumnorkaev and Gilchrist ${ }^{[113]}$ transferred the co-assembly concept to a horizontal deposition method and demonstrated the filling of a large sphere silica monolayer (diameter $1 \mu \mathrm{m}$ ) with a high number of small polystyrene latex particles (diameter $100 \mathrm{~nm}$ ). The amount of small particles was controlled by changing the relative concentration of large and small spheres. However, no stoichiometric configurations were produced. Only recently, a simple horizontal evaporation method was reported for the controlled co-assembly of binary crystals with various configurations, including stoichiometric $\mathrm{LS}_{2}$ and $\mathrm{LS}_{6}$ structures. ${ }^{[114]}$

The use of interface-mediated processes has only recently been identified as an attractive platform for the crystallization of binary monolayers. As the particles feature a high lateral mobility, sufficient time is provided for the small particles to find their minimum free energy position at the interstitial sites of the monolayers of large spheres. Yu and coworkers ${ }^{[115]}$ introduced a method for the direct assembly of binary structures at the air/water interface. They demonstrated a good control over interstitial site occupation and reported the formation of $\mathrm{LS}_{2}$ and $\mathrm{LS}_{6}$ configurations for polystyrene monolayers with size ratios between 0.147 and 0.194 . The use of a Langmuir trough for crystallization experiments was proposed by Detrich et al. in 2010. However, they did not achieve a proper ordering of binary silica particle systems. ${ }^{[116]}$ Yet, 
a precise control over the stoichiometry of binary monolayers co-assembled on a Langmuir trough has not been realized.

\subsection{Preparation of nanostructures on surfaces}

With the emergence of nanotechnology as a novel key player in science and technology in the 1990s, tremendous efforts have been initiated to prepare both dispersed nanoparticles as well as surface-bound nanostructures. In the focus of this thesis is the creation of complex nanostructures on surfaces. Thus, a short overview over common fabrication techniques for the creation of such structures shall be given in this chapter. In general, the different approaches can be classified according to a number of different parameters, including accessible shape of structures, materials to be employed, resolution, the surface area that can be patterned as well as time and costs of the process.

Here, the classification was chosen between serial and parallel processes. The difference can roughly be translated to expensive and slow versus fast and cheap. The former methods usually require expensive equipment that is used to create arbitrarily shaped nanostructures with a high precision and resolution. As the structures are created in a serial fashion, long times are typically required in the processes and the patterning of large areas is cumbersome if not impossible. Typical examples of such processes are electron beam lithography (eBeam), surface patterning with a focused ion beam (FIB) or dip-pen lithography that uses an AFM cantilever to write structures on substrates using "molecular inks" that selectively attach to surfaces. ${ }^{[17]}$

To make nanotechnology available to a broader range of researchers - and thus to support its use in a larger variety of applications - methods are required that are not dependent on sophisticated and expensive equipment. In approaches coined "simple nanotechnology" by George Whitesides ${ }^{[17]}$, several innovative ideas have been introduced to (partially) circumvent the drawbacks of serial processes.

The common feature of these approaches is the choice of masks that inherently possess patterns with nanometer dimensions that can subsequently be used to create nanostructures. The structural design that can be created is thus defined by the mask and cannot be arbitrarily chosen as in serial processes. However, this drawback is traded against a substantial decrease in production time and costs and usually, the patterning of arbitrarily large areas - defined only by the extensions of the mask - is readily achieved.

A number of different processes have been introduced and efforts have been undertaken to extend the accessible feature size and shape of the structures. The most prominent examples of 
such techniques are based on nano-phase separations of block copolymers (block-copolymer lithography) and the self-assembly of colloidal monolayers (colloidal lithography). Table 3.4.1. gives an overview of the most common nanopatterning techniques. The table also includes micellar and non-conventional colloidal lithography as two more exotic patterning methods that are relevant for this thesis and will be introduced below.

Table 3.4.1. Overview over different nanopatterning techniques

\begin{tabular}{|c|c|c|c|c|c|c|}
\hline Method & Principle & Time & Resolution & $\begin{array}{l}\text { Structure } \\
\text { variety }\end{array}$ & $\begin{array}{l}\text { Symmetry } \\
\text { of arrays }\end{array}$ & Refs. \\
\hline $\begin{array}{l}\text { eBeam } \\
\text { lithography }\end{array}$ & $\begin{array}{l}\text { electron beam } \\
\text { crosslinks photoresist }\end{array}$ & slow & $\begin{array}{l}\text { high } \\
(>10 \mathrm{~nm})\end{array}$ & High & $\begin{array}{l}\text { very high, } \\
\text { adjustable } \\
\text { symmetries }\end{array}$ & {$[117]$} \\
\hline $\begin{array}{l}\text { Focused ion } \\
\text { beam }\end{array}$ & $\begin{array}{l}\text { ion beam } \\
\text { etches/deposits } \\
\text { structures }\end{array}$ & slow & $\begin{array}{l}\text { high } \\
(>10 \mathrm{~nm})\end{array}$ & High & $\begin{array}{l}\text { very high, } \\
\text { adjustable } \\
\text { symmetries }\end{array}$ & [117] \\
\hline $\begin{array}{l}\text { Dip-pen } \\
\text { lithography }\end{array}$ & $\begin{array}{l}\text { AFM tip deposits } \\
\text { thiol "ink" }\end{array}$ & $\begin{array}{l}\text { very } \\
\text { slow }\end{array}$ & $\begin{array}{l}\text { high } \\
(>10 \mathrm{~nm})\end{array}$ & High & $\begin{array}{l}\text { very high, } \\
\text { adjustable } \\
\text { symmetries }\end{array}$ & {$[117]$} \\
\hline $\begin{array}{l}\text { Standard } \\
\text { UV-Photo- } \\
\text { lithography }\end{array}$ & $\begin{array}{l}\text { photons } \\
\text { crosslink/dissolve } \\
\text { resist }\end{array}$ & very fast & $\begin{array}{l}\text { very Low } \\
(>200 \mathrm{~nm})\end{array}$ & High & $\begin{array}{l}\text { very high, } \\
\text { adjustable } \\
\text { symmetries }\end{array}$ & {$[117]$} \\
\hline $\begin{array}{l}\text { Colloid } \\
\text { lithography }\end{array}$ & $\begin{array}{l}\text { colloid monolayer as } \\
\text { mask }\end{array}$ & very fast & $\begin{array}{l}\text { medium } \\
(>30 \mathrm{~nm})\end{array}$ & Limited & $\begin{array}{l}\text { high, } \\
\text { limited } \\
\text { primarily to } \\
\text { hexagonal }\end{array}$ & {$[4],[5]$} \\
\hline $\begin{array}{l}\text { Block- } \\
\text { copolymer } \\
\text { lithography }\end{array}$ & $\begin{array}{l}\text { phase separated block } \\
\text { copolymers as mask }\end{array}$ & fast & $\begin{array}{l}\text { high } \\
(>\sim 10 \mathrm{~nm})\end{array}$ & low & low & {$[118]$} \\
\hline $\begin{array}{l}\text { Micellar } \\
\text { lithography }\end{array}$ & $\begin{array}{l}\text { Me-containing } \\
\text { micelles as sacrificial } \\
\text { layer }\end{array}$ & very fast & $\begin{array}{l}\text { very high } \\
\text { (no lower } \\
\text { limit) }\end{array}$ & very low & low & $\begin{array}{l}{[119],} \\
{[120]}\end{array}$ \\
\hline $\begin{array}{l}\text { Non- } \\
\text { conventional } \\
\text { colloidal } \\
\text { lithography }\end{array}$ & $\begin{array}{l}\text { Me-containing } \\
\text { colloids as sacrifial } \\
\text { layer }\end{array}$ & very fast & $\begin{array}{l}\text { very high } \\
\text { (no lower } \\
\text { limit) }\end{array}$ & very low & high & {$[121]$} \\
\hline
\end{tabular}




\subsubsection{Colloidal lithography}

Most relevant for this thesis is the use of colloidal monolayers for the generation of nanostructures in a process usually termed colloidal lithography or nanosphere lithography. ${ }^{[5]}$ First reported by Fischer and Zingsheim in $1981,{ }^{[4]}$ colloidal lithography has matured into a widely-used technology and there are a number of reviews that highlight recent advances and developments. ${ }^{[9-10,122-124]}$

The process flow of classical colloidal lithography is illustrated in Figure 3.4.1. A close-packed colloidal monolayer is assembled onto a solid substrate (1) and gold is evaporated through the monolayer mask (2). As a result, triangular shaped gold nanostructures, reflecting the shape of the interstitial sites of the monolayer, are deposited on the substrate (3).
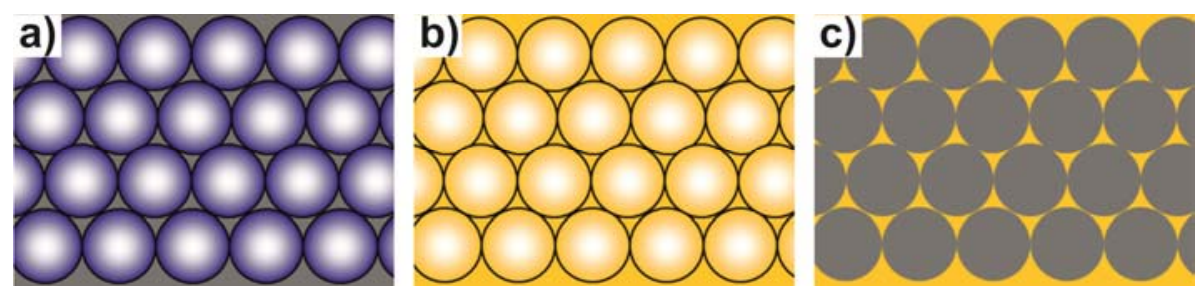

Figure 3.4.1. Classical colloidal lithography process leading to triangular shaped nanoparticle arrays. A colloidal monolayer is assembled (1) and used as a mask for the evaporation of a thin metal film (2). Subsequent removal of the mask yields arrays of triangular nanostructures (3) that reflect the shape of the interstitial sites of the monolayer.

Several years after the first report, Richard van Duyne and his group set out to explore scope and limitations of the fabrication process and investigated the intrinsic optical properties of such nanoscale metal particles (chapter 3.5). They used different metals and varied the size and shape of metal nanoparticle and managed to produce double and triple structures by multiple evaporation steps. ${ }^{[122,125-126]}$

In the last decade, focused research efforts were initiated that widened accessible structures by innovative combinations of angular dependent evaporation and etching steps. Thus, nanoscale discs, ${ }^{[127]}$ rings, ${ }^{[128]}$ ellipsoids ${ }^{[11]}$ and crescent shaped particles ${ }^{[129-130]}$ were successfully prepared as well as hole-films, ${ }^{[74,131]}$ nanobowls, ${ }^{[132]}$ nanoneedles, ${ }^{[14]}$ membranes ${ }^{[132]}$ and arrays of pillars of different materials. ${ }^{[133-135]}$

\subsubsection{Micellar lithography}

Micellar lithography as a more exotic lithographic method shall be briefly introduced here as the non-conventional lithography process described in this thesis (chapter 3.4.3) can be seen as 
an extension of the micellar technique. The micellar lithography process was introduced by Spatz and Moeller in 2000. ${ }^{[119]}$ They used block-copolymer micelles of polystyrene-poly(2vinylpyridine) that were charged with auric acid during the assembly process. After assembly into a two-dimensional crystal on a solid substrate, a plasma etching procedure was applied to remove the organic compounds and to reduce the auric acid to inorganic gold nanoparticles. Using this process, arrays of gold nanoparticles with a reasonable order reflecting the symmetry of the micellar monolayer are thus obtained. The size of the gold nanoparticles is controlled by varying the amount of auric acid incorporated in the core of the block-copolymer micelles while the interparticle distance can be adjusted by chosing polymers with different molecular weights. Kaestle and Spatz demonstrated the preparation of $\mathrm{Au}$ nanoparticle arrays with dot sizes between 1 and $15 \mathrm{~nm}$ and interparticle distances between $25 \mathrm{~nm}$ and $140 \mathrm{~nm} \cdot{ }^{[120]}$ In contrast to block-copolymer lithography, the micellar lithography process does not use the micelles as mask for the preparation of surface nanopatterns but as a sacrificial layer to determine the symmetry and lattice constants of the gold nanoparticles.

\subsubsection{Non-conventional colloidal lithography}

The micellar lithography technique was modified to a process that will be referred to as nonconventional colloidal lithography to facilitate distinguishing to the conventional colloidal lithography approach. Introduced by Plettl et al. in $2007,{ }^{[121]}$ the process applies metal-complex containing latex particles in a similar approach as the micellar technique. Figure 3.4.2 shows the process schematically. A monolayer of metal-complex containing latex particles is assembled on a substrate, subjected to oxygen plasma to combust the organic material and finally annealed to produce ordered arrays of spherical metal nanoparticles.

Compared to the micellar approach, the use of colloids has two distinct advantages. First, the long range order in a colloidal monolayer is substantially higher than in micellar monolayers due to the higher homogeneity and the hardness of the colloid spheres. Second, the size range of obtainable lattice constants can be further increased as the diameter of micelles is limited to sizes below approximately $150 \mathrm{~nm}$. Both approaches feature one highly interesting property: The process does not have an inherent lower limit in size of the metal nanoparticles created, as very little amounts of metal complexes - or salts in the case of micelles - can be incorporated without problems. Thus, it possesses a very high potential in applications that require extremely small particle sizes. 


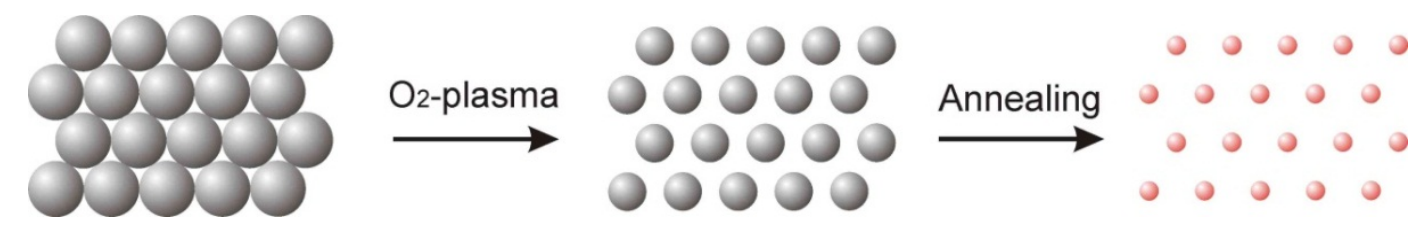

Figure 3.4.2. Non-conventional colloidal lithography

\subsubsection{Applications of colloidal monolayers and surface nanostructures fabricated by colloidal lithography}

There is a great variety of applications for nanostructures created by colloidal lithography as an increasing number of technologies require ordered patterns with nanoscale dimensions. Depending on the application, different properties of the structures created by colloidal lithography can be in the focus.

The first and most prominent applications of colloidal lithography exploit the plasmonic properties of metal nanostructures that were extensively used for the detection of changes in the dielectric environment and thus detect binding events, e.g., of biomolecules. ${ }^{[24]}$

In the next chapter, a more detailed description of the plasmonic properties of metal nanostructures will be given. Similarly, the strong near-fields accompanying plasmonic resonances have also been used for the amplification of the fluorescence of dye molecules ${ }^{[11]}$ and in surface-enhanced Raman spectroscopy. ${ }^{[12]}$ Even more complex applications of the plasmonic resonances in metal nanostructures are discussed. For example, the split ringanalogous geometry of nano-crescent is discussed to be a valuable building block for the preparation of metamaterials with optical properties not occurring in nature, as for example a negative index of refraction. In such structures, the incident light excites circulating currents leading to local magnetic dipole moments that can counteract the incident magnetic field and result in negative permeability, a prerequisite for negative refraction. ${ }^{[136]}$

The second set of applications makes use of the high degree of order of colloidal monolayers. Most predominantly, this order can be transferred to pattern the substrate in a much defined way. Thus, patterning of intrinsically interesting materials with nanoscale dimensions has been achieved; including graphene, ${ }^{[137]}$ carbon nanotubes ${ }^{[138]}$ or proteins. ${ }^{[139]}$

The creation of ordered arrays of pillars in conventional substrate materials as silicon or glass induces superhydrophobicity ${ }^{[14]}$ and has been used to prepare antireflective ${ }^{[140]}$ and self-cleaning materials. $^{[15]}$

The nanoscale periodicity has also been shown to influence cell adhesion that switched from adhesive to non-adhesive in dependence on the order and spacing in nanoscale gold arrays. ${ }^{[16]}$ 
The photonic properties of colloidal monolayers have attracted attention as they can be used for the bioinspired mimic of the structural color in butterfly wings. ${ }^{[13]}$

Finally, when assembled on an interface and infiltrated with polymerizable monomers, colloidal monolayers have been used for the creation of membranes featuring defined pore sized with nanometer dimensions. ${ }^{[141]}$

\subsection{Localized surface plasmon resonances: a fundamental properties of metallic nanostructures}

The interaction of electromagnetic radiation with metals causes a collective oscillation of the free electron gas at the resonance frequency. This collective oscillation is termed plasmon. Depending on the size and dimensions of the metal, different types of plasmons can be excited. Volume plasmons occur in three-dimensional metals, two-dimensional thin metal films support surface plasmons and the confinement of the electron gas in metal nanoparticles leads to apparition of particle plasmons.

The most prominent example of plasmons in every-day life is the bright color of church windows that is caused by gold nanoparticles embedded in the glass matrix.

Academically, plasmon resonances have attracted attention, especially due to their sensitivity to changes of the dielectric constant in close vicinity of the metal structure. Changes in the direct environment of a nanostructure, e.g., upon binding events, are thus detected by a shift of the plasmon resonance wavelength.

As parts of this thesis present the use colloids to create and characterize the plamonic properties of metal nanostructures, the excitation of plasmon resonances in such confined structures by electromagnetic fields and the properties of the plasmons will be briefly introduced in the following.

\subsubsection{Localized surface plasmon resonances}

Particle plasmons can be excited in metal nanostructures. In contrast to bulk metals or thin films, the electron gas of the metal is strongly confined in all three dimensions within a structure that is smaller than the wavelength of the incident light. Due to its small size, the nanostructure can be penetrated by the electric field of the incident light. The field inside the nanostructures 
shifts the free electrons collectively with respect to the fixed positively charged atom cores in the crystal lattice. Electrostatic attraction between the separated charges leads to a restoring force. The surface charges thus alternate in time and form an oscillating dipole that radiates electromagnetic waves. Strong absorption and scattering phenomena arise when the frequency of the incident light field is in resonance with the eigenfrequency of the electron gas oscillation. In the simplest case, a dipolar oscillation is thus induced which is schematically illustrated in Figure 3.5.1.

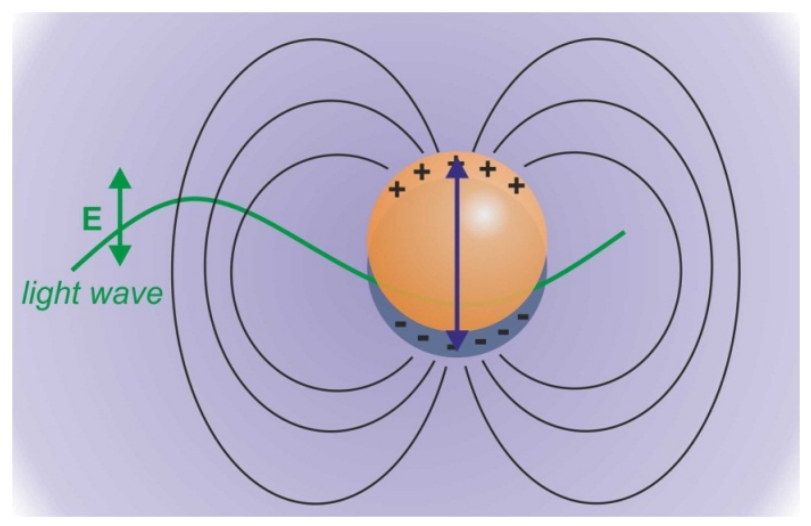

Figure 3.5.1. Schematic representation of the excitation of a localized surface plasmon resonance in a metal nanoparticle by an incident electromagnetic wave. Figure adopted from Raschke. ${ }^{[142]}$

The resonance frequency of particle plasmons strongly depends on the metal, shape, size, and dielectric environment of the nanoparticle as these factors determine the extent of charge separation during the oscillation. Variation of the geometrical parameters enables the precise tailoring of the resonances from the whole visible range far into the infrared.

The resonance oscillation in the nanostructure also results in an accumulation of electromagnetic field lines that can lead to highly localized, strongly enhanced near-fields, especially if the geometry features sharp tips or edges.

The theoretical description of particle plasmons is based on two distinct properties: the small size and the material properties of the metal.

A simple model for the dielectric properties of metals was introduced by Drude in $1900 .{ }^{[143]}$ The model describes the conduction electrons in metals as a classic electron gas that executes a diffusive motion. The electron motion is damped by mutual collisions which occur with an average rate of $1 / \tau$ ( $\tau$ being the electron relaxation time of the free electron gas). If an electric field is applied, the electrons start to oscillate in response. From this simple model, a description of the frequency-dependent dielectric function $\varepsilon(\omega)$ of the Drude metal under the influence of an electromagnetic field can be derived: ${ }^{[144]}$ 


$$
\varepsilon(\omega)=\varepsilon_{\infty}-\frac{\omega_{p}{ }^{2}}{\omega^{2}+i \frac{\omega}{\tau}}
$$

In the equation, the plasma frequency $\omega_{p}$ describes the oscillation of the free electron plasma:

$$
\omega_{p}=\sqrt{\frac{n_{e} e^{2}}{\varepsilon_{0} m_{e}}}
$$

with $n_{e}$ being the electron density of the metal, $e$ the electron charge, $\varepsilon_{0}$ the vacuum permitticity and $m_{e}$ the effective mass of the conducting electrons. $\varepsilon_{\infty}$ describes the contribution of the bound electrons to the polarizability. If only conduction band electrons contribute to the dielectric function, $\varepsilon_{\infty}$ should have the value of 1 .

The simple Drude model accurately describes most of the dielectric properties of metals. However, the free-electron model often breaks down at optical frequencies, especially for noble metals (e.g. gold, silver). This is related to the excitation of stronger bound d-band electrons into the conduction band (interband damping) that is not accounted for in the Drude model. ${ }^{[144-145]}$ Optical resonances are supported by metallic nanostructures with sizes in the range of the wavelength of light or below. The response of a metal particle to an external electric field, leading to absorption and scattering can be analytically treated by Mie Theory. ${ }^{[146]}$

For small metal particles $\left(d_{(\text {particle })}<\lambda\right)$, the phase of the incident light experienced by the particle can be treated to be constant (Figure 3.5.2). This approximation, known as quasi static approximation, was introduced by Lord Rayleigh and yields reasonable results for particles with sizes below $40 \mathrm{~nm}^{[145]}$

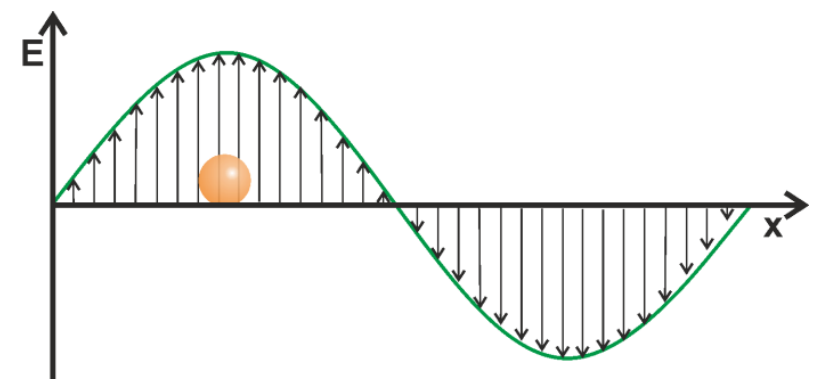

Figure 3.5.2. Not-to-scale picture of the conditions for the quasi-static approximation. The size of the particle is much smaller than the wavelength of the incident light. Figure adopted from Zins. ${ }^{[147]}$ 
The central result of the quasi static approximation is the expression of the microscopic polarizability $\alpha$ of a small sphere with radius $r$ that is linked to the macroscopic dielectric properties of both particle $(\varepsilon)$ and surrounding medium $\left(\varepsilon_{m e d}\right):^{[144]}$

$$
\alpha(\omega)=4 \pi r^{3} \frac{\varepsilon-\varepsilon_{m e d}}{\varepsilon+2 \varepsilon_{m e d}}
$$

The function, known as Clausius-Mosotti relation, yields a maximum of $\alpha$ for $\varepsilon=-2 \varepsilon_{\text {med }}$. As the dielectric function of the metal is frequency-dependent, the resonance condition is fulfilled for a distinct frequency determined by the materials' property and the dielectric function of the surrounding medium. For materials with a small imaginary part of $\varepsilon$, the resonance condition simplifies to:

$$
\operatorname{Re}[\varepsilon]=-2 \varepsilon_{\text {med }}
$$

This so-called Fröhlich criterion directly shows the sensitivity of the plasmon resonance frequency towards changes in the dielectric constant of the medium. It is this special property that is the base for the most-widespread application of metallic nanoparticles: the sensing of minute changes in its direct environment.

\subsubsection{Sensing with localized surface plasmons}

As described above, the particle plasmons are very sensitive to changes in the dielectric environment of the nanostructures. An increase in refractive index around the particles, e.g. due to binding of molecules to the structure, induces a red-shift of the plasmon resonance that can be directly monitored by UV/Vis spectroscopy. Figure 3.5.3 presents two typical sensing experiments. (a) Shows a metal nanostructure that is covered with a thin film. The plasmon resonance shifts to lower energies after deposition of the thin film. A biosensing experiment is illustrated in Figure 3.5.3b. The nanostructure is first functionalized with molecules that possess high selectivity and strong binding interactions with the targeted molecules to be detected. As a typical example, the functionalization of the nanostructures with antibodies is depicted. The surface functionalization induces a first red-shift of the resonances. Finally, the sensing platform is immersed in the analyte solution that contains the respective antigens. Selective binding to the antibodies immobilized onto the nanostructure takes place and further alters the dielectric environment. The shift of the plasmon can then be used to detect the binding events. 
This concept has been realized for a great variety of materials, including the assemble of thiole monolayers ${ }^{[46-47]}$ and polymer thin films, ${ }^{[148]}$ the swelling behavior of hydrogels ${ }^{[149]}$ and the detection of biologically relevant molecules. ${ }^{[45,150]}$

a)
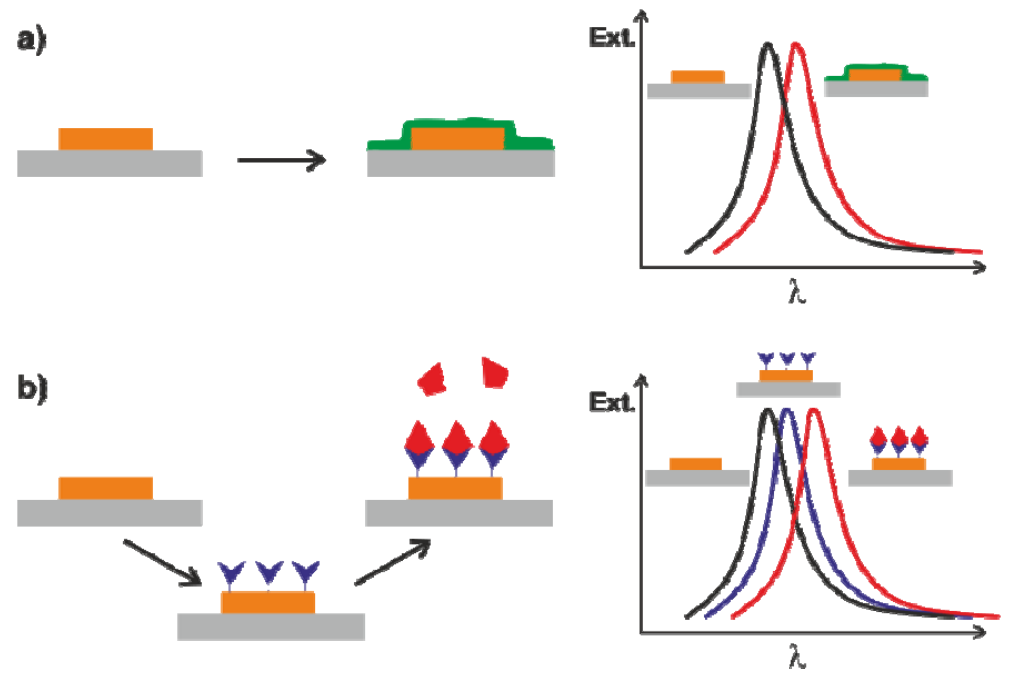

Figure 3.5.3. Sensing with localized surfaces plasmons. a,b) schematic illustration of a metal nanostructure in a sensing experiment. a) Coverage of the nanostructure with a thin film. b) A typical biosensing experiment: the nanostructure is first functionalized with a functional molecule (e.g. antibody) that selectively binds to a second molecule (e.g. antigen) that can thus be detected in an analyte solution. $c, d)$ Resulting shift of the plasmon resonances upon coverage of the structure or binding of the molecules.

\subsubsection{Plasmon hybridization}

Localized surface plasmon resonances are also sensitive to the presence of additional metal nanostructures: The placement of a second nanoparticle in close vicinity to a first one imposes drastic changes of the resonance position. This is caused by a coupling process of the individual plasmon resonances. An intuitive concept for the description of such nanoparticle dimers has been elaborated by the group of Peter Nordlander. ${ }^{[151-152]}$ They suggested treating the coupling of resonances as an electromagnetic analogue to molecular orbital theory: the plasmonic resonances of two nanostructures in close proximity to each other undergo a coupling process that gives rise to new resonances as linear superpositions of the original resonances. In this picture, the two particles can be described as a plasmonic molecule, ${ }^{[152]}$ similar to two atoms chemically bound to form a molecule. As the wave function of these atoms can be superimposed to give rise to a binding- and an antibinding molecular orbital in the molecule, the plasmonic modes hybridize as well. The shift of the hybridized resonances with respect to the parental resonances of single objects can thus be explained by simple geometric arguments as illustrated in Figure 3.5.4. 

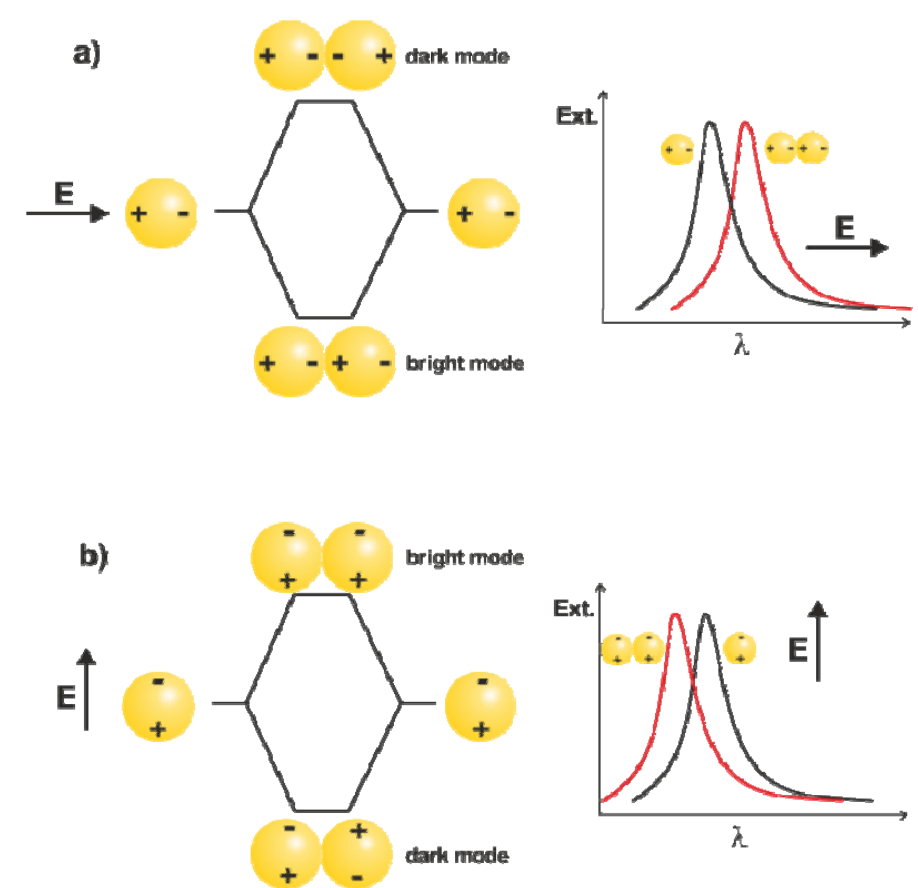

Figure 3.5.4. Plasmon hybridization in nanoparticle dimers. a) Hybridization scheme and observed shifts of the plasmon modes for an electric field parallel to the inter-particle axis; b) scheme and plasmon shift upon hybridization for an electric field perpendicular to the inter-particle axis.

Two spherical nanoparticles are placed in close proximity. Depending on the orientation of the dimer with respect to the polarized incident electric field, two hybridization scenarios can be envisioned. An electric field vector parallel to the inter-particle axis gives rise to a hybridization scenario as shown in (a): the linear combination of the two individual resonances yields a symmetric mode with both dipoles oscillating in phase and an antisymmetric mode with an outof-phase oscillation. The energy of the symmetric mode is reduced. Of the two hybrid resonances, only the symmetric mode possesses a dipole that can interact with the incident light. Thus, this mode is excited in normal illumination and therefore termed bright mode. The antisymmetric mode, however, cannot couple efficiently to the external electric field as it does not feature a dipole moment. It is not excited by the external field and called a dark mode.

The hybridization model for an electric field vector perpendicular to the inter-particle axis is shown in Figure 3.5.4b. Again, the parental plasmon mode can be combined symmetrically and antisymmetrically. However, due to the close proximity of like-charges, the symmetric in-phase oscillation is higher in energy compared to the out-of-phase oscillation of the antisymmetric mode. In analogy to before, only the symmetric mode features a dipole and is excited by the electric field while the antisymmetric combination is a dark mode. Thus, a blue-shift of the resonances is detected if the position of the dimer is fixed with respect to the electric field.

If the electric field is not polarized or the nanoparticle dimers can freely rotate, both bright hybrid modes are excited and two plasmons with higher and lower energy compared to the resonance of a single particle are observed. 


\section{Characterization methods}

\subsection{Atomic force microscopy (AFM)}

Atomic force microscopy ${ }^{[153]}$ (AFM) is a powerful tool to image and characterize the topography of a surface. In contrast to optical imaging methods, the atomic force microscope is not limited by the diffraction limit and imaging with sub-nanometer resolution is feasible. Additionally, the atomic force microscope provides images with exact dimensions as the contrast purely results from height differences of the sample. Thus, it is used to very precisely determine the dimensions of a sample.

The basic principle of the microscope is shown in Figure 4.1.1. The cantilever tip is placed on or very close to the surface. A laser beam is focused onto the cantilever and reflected into the center of a photodiode. The sample is placed on a piezo-crystal that is able to move in $\mathrm{x}, \mathrm{y}$ and $\mathrm{z}$ direction. The lateral movement of the stage is used to scan the sample in the desired area. The surface topogrography results in a deflection of the cantilever. This deflection causes the reflected laser beam to move on the photodetector as well can thus be read-out electronically. An electronic feedback control is used to keep the cantilever constant by adjusting the $\mathrm{z}$ position of the sample in response to the deflection of the cantilever. The height image produced by an atomic force microscope visualizes the z-movement of the stage.

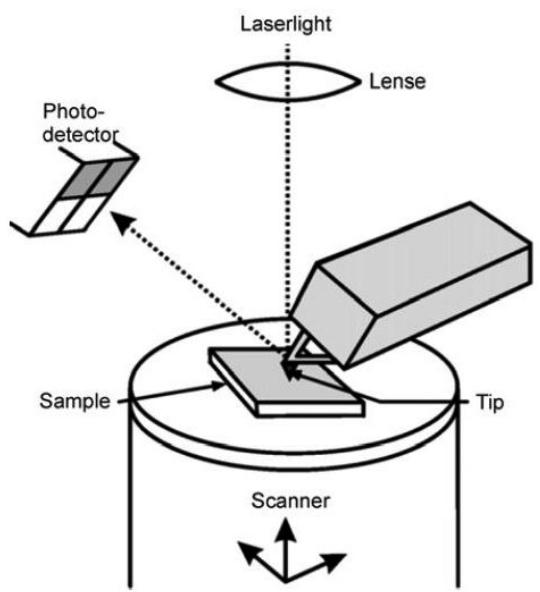

Figure 4.1.1. Schematic illustration of an atomic force microscope. Laser light is focused on the cantilever and reflected onto a photodetector. Deflections of the cantilever caused by the surface topography translate into a deflection of the laser beam that is detected by the photodetector. The stage is mounted on a piezo crystal that can move in all space directions. The image is taken from the textbook of Butt. ${ }^{[34]}$ 
There are two main operation modes for an atomic force microscope. In contact mode, the tip is in permanent contact with the surface. This mode achieves a high resolution but can damage or deform the sample due to the high pressure of the tip caused by the small contact area. For softer samples, a tapping mode is often applied. In this mode, the cantilever is vibrated at its resonance frequency. When such a vibrating cantilever approaches a surface, the vibration amplitude will start to decrease once the tip hits the surface. The scanning is then operated at a constant reduction of the vibration amplitude instead of a constant deflection. As a result, the cantilever only touches the surface shortly and is not in permanent contact. Thus, less damage is induced at the sample surface. Another benefit of the tapping mode is the possibility to visualize the phase difference of the resonance vibration. Depending on the "stickiness" of the surface, the contact time of the cantilever differs for different sample materials and thus, is released with a phase difference. This can be used to determine mechanical properties of the sample and visualizes e.g. different surface materials. For more details on the atomic force microscope and more sophisticated techniques, the textbook of Butt ${ }^{[34]}$ and references therein are recommended.

\subsection{Inductively coupled plasma optical emission spectrometry (ICP-OES)}

The inductively coupled plasma optical emission spectrometry ${ }^{[154]}$ is an analytical method for the detection of chemical elements with great precision. The resolution depends on the respective elements and sample conditions and reaches limits of several $\mu \mathrm{g} \cdot \mathrm{l}^{-1}$. ICP-OES is widely used for the quantitative elemental analysis in food sciences (i.e. detection of ions in drinking water), environmental analysis (detection of toxic elements) and in metallurgy (verification of alloy compositions and metal recycling).

Traditionally, ICP-OES is optimized for the detection of metal ions in aqueous solutions and only few examples report on the detection of ions in colloidal systems, mostly in geological applications for the detection of minerals in sediments. ${ }^{[155-157]}$ 


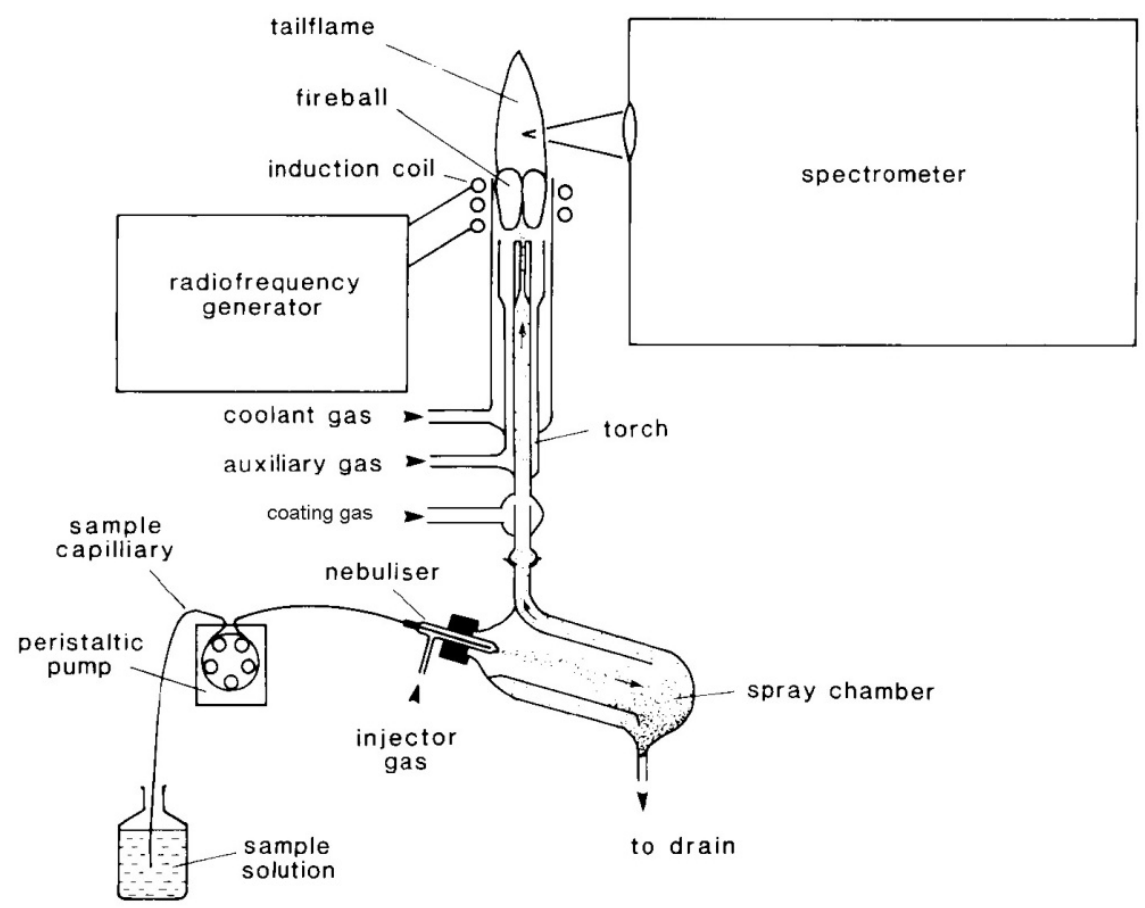

Figure 4.2.1. Schematic illustration of an inductively coupled plasma optical emission spectrometer.

Figure 4.2.1 schematically shows the main parts of an ICP-OE spectrometer. The liquid sample is transported to the nebulizer by a peristaltic pump. The nebulizer converts the liquid into an aerosol and injects it into the cyclone chamber that transports it into the plasma torch. The plasma torch is the heart of the instrument. Usually, Argon is used as plasma gas due to its lower price and lower ionization energy compared to helium or neon. The argon atoms are ionized by means of a Tesla spark and energy is coupled into the gas by an alternating highfrequency electromagnetic field. The field accelerates ions and electrons and the plasma heats up by mutual collisions of the charge carriers. The temperature in an inductively coupled plasma reached $6,000-10,000 \mathrm{~K}$.

The aerosol reaching the plasma quickly passes all aggregate states: it solidifies upon drying, melts, vaporizes and is finally ionized by the carrier gas energy. The analyte ions present in the plasma are subsequently excited and emit photons with characteristic wavelengths (spectral lines). These lines are detected by the optics in the spectrometer. As the individual lines are characteristic for each element, several elements can be detected simultaneously in one sample. For a quantitative analysis of the elemental composition, a calibration is needed and recorded by aqueous solutions of the respective elements in known concentrations. For a reliable determination of the element concentration, the standards need to resemble the analyte solution (matrix matching) ${ }^{[158]}$ For a detailed description of the individual components as well as measurement possibilities and pitfalls, the textbook by Nölte ${ }^{[159]}$ is highly recommended. 
To quantify the composition of materials, inductively coupled plasma based spectrometric methods ${ }^{[154,160]}$ such as mass spectrometry (ICP-MS) or optical emission spectrometry (ICPOES) are very powerful tools and are widely used for the quality control in different industrial sectors, ranging from metal and steel engineering or noble metal recycling to food control and environmental analysis, as the determination and quantification of (toxic) metal ions in drinking water. Traditionally, ICP methods are optimized for use the detection of metal ions in aqueous solutions. ${ }^{[158]}$ A number of reports on the use of ICP for the characterization of colloidal systems can be found in literature. While some reports describe the elemental analysis of synthetic, inorganic particles, ${ }^{[161-164]}$ most of the literature reported for ICP characterization of colloids deals with slurry methods for the analysis of sediments for environmental sciences. ${ }^{[155-157]}$ Investigations on polymer-based hybrid nanoparticles are rare in literature. However, Vancaeyzeele et al. used ICP-MS as well as ICP-OES for the determination of lanthanide complexes in latex particles but reported lanthanide contents systemically lower than the value expected without finding an explanation for this feature of data. ${ }^{[165]}$

\subsection{Two-dimensional chemistry and physics on a Langmuir trough}

In 1917, Irving Langmuir recognized that molecules at the surface of a liquid film experience different forces than molecules in the bulk liquid: ${ }^{[166]}$ the chemistry and phyiscs in two dimensions was born. He developed a simple apparatus that became known as Langmuir trough to study the behavior of molecules at the interface for which he was awarded the Nobel Prize in 1932. The trough, illustrated in Figure 4.3.1, is usually made of Teflon to allow and is equipped with two barriers that can be moved to compress material present at the surface and a balance to monitor the surface pressure (see below). 


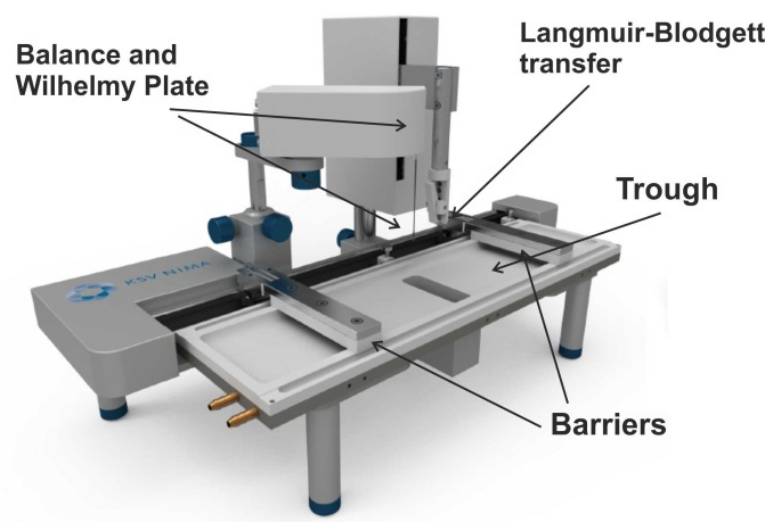

Figure 4.3.1. Photograph of a Langmuir trough. Taken from KSV-NIMA product catalogue.

When amphiphilic molecules are applied to the air/water interface (or oil-water interface), they will orient in a way that their hydrophilic head groups reside in the water phase while the hydrophobic tails will stick out of the water into the air (oil) phase. If such molecules are present on the air/water interface on a Langmuir trough and compressed, the interfacial area available for one molecule will be continuously reduced. As a consequence, the molecules start to interact and exert repulsive forces on each other. These forces result in a two-dimensional analogue of a pressure that is called surface pressure $\Pi$. It is equal to the reduction of the surface tension of the pure liquid by the film of amphiphilic molecules:

$$
\Pi=\gamma_{0}-\gamma
$$

where $\gamma_{0}$ denotes the surface tension of the pure liquid and $\gamma$ the tension of the monomolecular film at the interface. For the investigation of surface active substances, surface-pressure-area isotherms are recorded that plot the pressure against the total surface area or the area available for a molecule at constant temperature.

By the very nature of the interface, the film thickness cannot exceed the height of one molecule and is a truly two-dimensional system; usually referred to as monolayer.

The surface pressure is recorded by the Wilhelmy-plate method: the forces resulting from the surface molecules are measured by a thin platinum or paper plate that is partially immersed into the water phase. The plate is connected to a sensitive balance that records changes of the surface tension of the water phase.

Several methods to transfer monomolecular films from the air/water interface to solid substrates have been proposed and are illustrated in Figure 4.3.2; most prominently Langmuir-Blodgett transfer ${ }^{[167-168]}$ (a) and Langmuir-Schaefer transfer ${ }^{[169]}(\mathrm{b})$. 

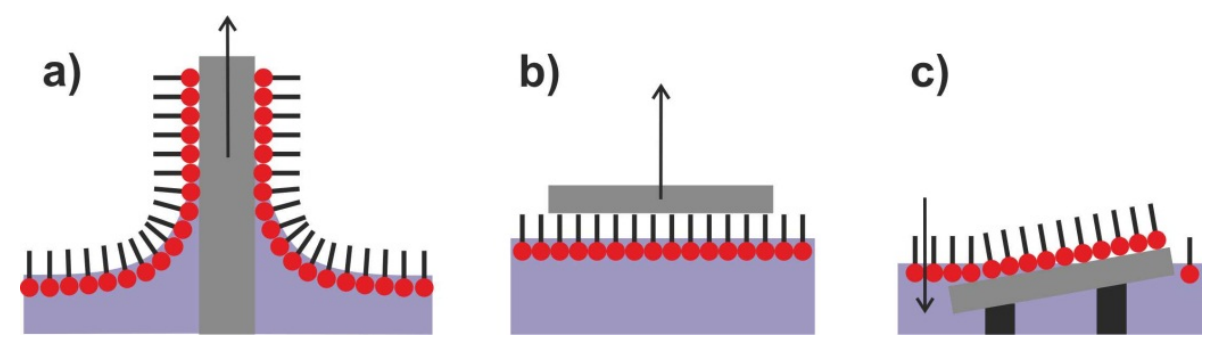

Figure 4.3.2. Transfer of a molecular monolayer to a solid substrate: a) Langmuir-Blodgett transfer; b) Langmuir-Schaefer transfer, c) surface lowering transfer.

In this thesis, a Langmuir trough is used to assemble colloidal particles at the air/water interface. The transfer is performed by lowering of the water level onto a substrate immersed in the subphase (Figure 4.3.2c).

\subsection{Scanning electron microscopy (SEM)}

Electron microscopy is the standard technique to characterize nanostructures microscopically. In contrast to an optical microscope, the electron microscope applies accelerated electrons as electromagnetic irradiation for the imaging process. The wavelength of such electrons is given by the de-Broglie relation that connects the wavelength of an object $\lambda$ with the Planck constant $h$ and its momentum $p$, that is the product of mass $m$ and the kinetic energy $E_{k i n}$ of the object:

$$
\lambda=\frac{h}{p}=\frac{h}{\sqrt{2 m E_{\text {kin }}}}
$$

Depending on the acceleration voltage, the resulting wavelengths of the electrons are below $0.1 \mathrm{~nm} \cdot{ }^{[34]}$ As such electrons possess a much smaller wavelength compared to visible light, the visualization of much smaller objects at the nanoscale is possible. The increased resolution is given by the Abbé criterion that defines the minimum distance $d$ between two points that can be resolved in dependency on the numerical aperture (NA) of the objective and the wavelength $\lambda$ of the radiation used:

$$
d=\frac{\lambda}{2 \cdot N A}
$$

For the imaging surface-bound nanostructures, the scanning electron microscope ${ }^{[170]}$ (SEM) is the method of choice. It focuses the electron beam onto the sample surface and scans pixel by pixel to create an image. The contrast in an electron microscopy image is given by the number of electrons reaching the detector that are recorded for every pixel during the scanning process. 
The final image is thus a two-dimensional map of the electron density distribution. The interaction of the electron beam with the sample surface is complex and several processes take place simultaneously. Figure 4.4.1 shows a schematic illustration of the different interaction processes. Secondary electrons $(\mathrm{SE})$ are low energy electrons $(<50 \mathrm{eV})$ that are ejected from the sample atoms by interaction with the primary beam. Due to their low energy, they can only leave the sample at very small distances from their generation place. Thus, they are typically used for the imaging process and lead to a high resolution both laterally and in depth. The threedimensional appearance of SEM images is caused by a higher emission of electrons at edges or sharp peaks of the surface known as "edge effect". Backscattered electrons (BE) are high energy electrons that result from elastic scattering of the beam electrons at the sample atoms. The intensity of backscattered electrons is very sensitive to the atomic number of the sample and can thus be used to visualize material contrasts. The detection of Auger electrons (AE) or characterstic X-ray radiation is possible with an electron microscope as well. This radiation is emitted when an electron is removed from the inner shells of an atom by the beam electrons and an electron from a lower-energy orbital falls back into the vacancy created by the first electron. This technique is termed energy dispersive $\mathrm{x}$-ray spectroscopy (EDX). ${ }^{[171]}$ Alternatively, the released energy can be taken up by a third electron that subsequently leaves the atom (Auger process).

For more details on electron microscopy, the textbook of Goldstein is recommended. ${ }^{[171]}$

a)

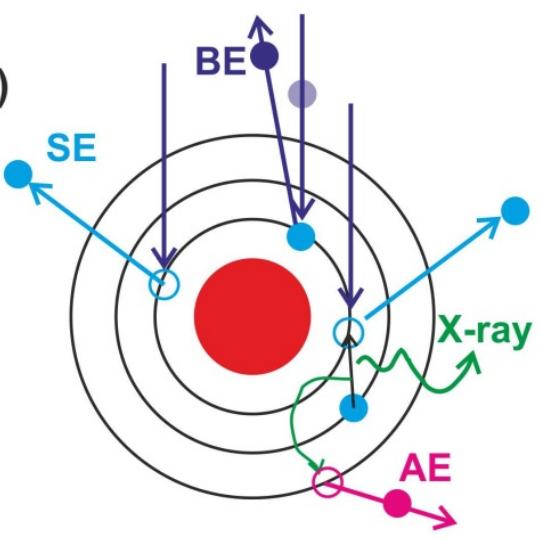

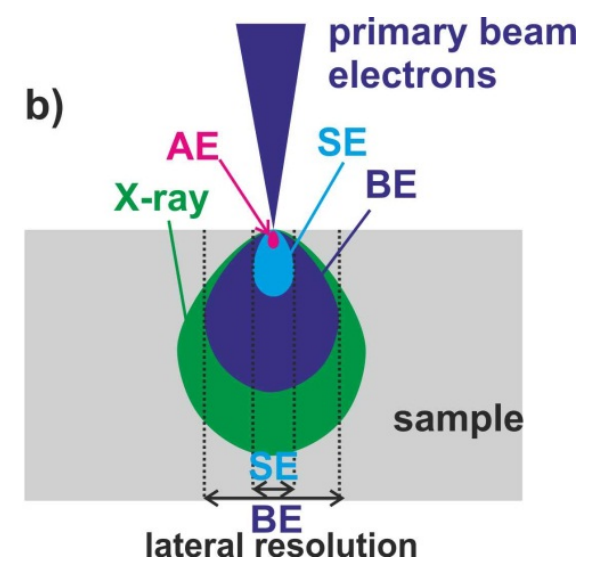

Figure 4.4.1. Interaction of the electron beam with the sample surface. a) Different interactions between primary beam electrons and an atom on the surface. b) Penetration depth and lateral extensions of the different processes. All relevant processes are specified in the text body. 


\subsection{Spinning drop tensiometry}

The spinning drop tensiometry technique measures the interfacial tension between two immiscible phases by analysis of the dimensions and shape of a droplet of one lighter phase centered in a second, continuous and denser phase in a rotating capillary tube. ${ }^{[172]}$ At high rotation speeds, the droplet deforms into a cylindrical shape. The profile of this droplet is analyzed by a video camera and can be directly used to calculate the interfacial tension between the two phases 1 and 2 using Vonnegut's equation: ${ }^{[173]}$

$$
\gamma_{1,2}=\frac{1}{4}\left(\rho_{2}-\rho_{1}\right) \omega^{2} R_{1}^{3}
$$

With a cyclindrical shape, the interfacial tension between the two liquids, $\gamma_{1,2}$, only depends on the difference in density of continuous $\left(\rho_{2}\right)$ and dispersed phase $\left(\rho_{1}\right)$, the rotational speed $\omega$ and the radius $R_{l}$ of the cylinder formed by phase 1 in phase 2 . 


\section{Results and Discussion}

The results of the different projects pursued for this thesis will be presented in the following.

The first part of the results and discussion chapter describes the synthesis and characterization of metal-complex containing polystyrene particles by miniemulsion and emulsion polymerization. A measurement protocol for the reliable determination of the metal content of latex particles by inductively coupled plasma optical emission spectrometry is established first (chapter 5.1.1). Subsequently, the synthesis and encapsulation efficiency of metal- $\beta$-diketonate complexes by emulsion polymerization is discussed exemplarily for platinum(II)cetylacetonate as complex compound (chapter 5.1.2.2.1). Next, an assessment of the stoichiometric encapsulation of both iron(III)acetylacetonate and platinum(II)acetylacetonate to yield multifunctional latex particles with defined composition by miniemulsion polymerization is performed (chapter 5.1.2.4.1). Finally, a seeded emulsion polymerization process is applied to control the size of the metal-containing latex particles and relevant reaction parameters are investigated (chapter 5.1.2.5). The application of metal-complex containing latexes in a nonconventional lithography approach will be described further below.

The next part covers methods for the crystallization of colloidal particles into different twodimensional architectures. First, a convenient process to produce highly ordered, single-sized particle monolayers using a direct assembly at the air/water interface is presented (chapter 5.2.1). Next, these monolayers are subjected to a plasma treatment to systematically reduce the size of the monolayer without affecting the lateral order. Thus, non-close-packed monolayers are created (chapter 5.2.2) Finally, the air/water interface on a Langmuir trough is used to prepare binary monolayers with defined configurations over large size ratios of the colloids by precisely adjusting the stoichiometry of large and small colloids at the interface (chapter 5.2.3).

In the final part, lithographic processes to fabricate nanostructure arrays from two-dimensional colloidal crystals are presented. The first approach to be presented utilizes metal-complex containing latex particles for a process coined non-conventional lithography (chapter 5.3.1). The metal-complex containing latex particles, introduced in the synthesis part, are crystallized into a monolayer and subjected to plasma treatment in order to combust the organic materials of the colloids. The remaining "crude" inorganic particles are subsequently annealed to singlecrystalline metallic particles. Using colloids with different metal contents and diameters, a precise adjustment of size and interparticle distance is demonstrated for platinum nanoparticle 
arrays. A step towards functional particle arrays is subsequently presented that described the transformation of the miniemulsion particles containing stoichiometric amounts of iron- and platinum-complexes into arrays of magnetic FePt particles.

Next, non-close-packed architectures are used to create crescent-shaped particles arrays. In the beginning, a short introduction to the preparation and optical properties of arrays of individual nano-crescents is given (chapter 5.3.2.1). In the following, a process for the construction of arrays of vertically stacked crescent dimer units that are separated by a thin insulating layer of several nanometers (chapter 5.3.2.2). The vertical alignment of nanostructures into quasi-3D architectures has not yet been demonstrated by colloidal lithography and allows for the observation of strong coupling processes between the individual crescents that are investigated experimentally and by means of computer simulations. Finally, the crescent dimer architecture is altered from a vertical stacking to an in-plane configuration using a different lithography protocol (chapter 5.3.2.3). The dimer units consisting of two opposing crescents in close proximity with tips facing each lead to coupling of the plasmon modes as well. In contrast to the stacked architecture, these structures feature an accessible "hot spot" in the small gap between the crescent tips that provides strongly enhanced electrical near-fields at the resonance wavelength of the coupled plasmon mode.

Finally, surface-embedded nanoparticle arrays are presented. The construction process is based on a template-stripping procedure and has been previously developed. As a continuation of this work, the high stability of this nanostructure architecture is exploited for the development of a robust sensing platform that can be mechanically cleaned and is re-usable (chapter 5.3.3.2). Furthermore, the surface of the patterned substrate features an extremely low surface roughness and a smooth transition between the different surface materials due to the preparation process. This remarkable property is exploited in a project to create nanoscale diffusion barriers in a solid supported lipid bilayer membrane by self-assembly and polymerization of surfaceanchored polymerizable artificial lipids (chapter 5.3.3.3). As the substrate is nearly topographyless, the diffusion barriers merge homogenously into a fluid lipid bilayer membrane and fluorescence recovery after photobleaching experiments indicate a closed distal bilayer formed on top of the diffusion barriers. The architecture can be used to control the diffusional velocity of the lipids or to confine lipids to designed spots on the substrate. 


\subsection{Particle synthesis and characterization}

\subsubsection{Accurate elemental analysis of metal-containing polymer latexes using inductively coupled plasma - optical emission spectrometry}

In this chapter, a detailed study on the use of inductively coupled plasma optical emission spectrometry (ICP-OES) to determine the metal content of metal complex-containing colloidal dispersions is presented.

As a major part of this thesis, the non-conventional colloidal lithography process (chapter 3.4.3) is applied to convert platinum- and iron/platinum containing colloidal particles into arrays of metal nanoparticles. The reliable determination of the metal content of these colloid samples is the key prerequisite for a proper analysis of the process.

However, it was found that a simple IPC-OES measurement of plain metal-complex containing colloidal dispersions generally gave rise to apparent metal contents significantly below the expected value. In order to examine the behavior of the colloidal dispersions in the ICP spectrometer, a systematic study on measurement conditions was performed; including variations of device parameters and different sample preparation strategies. It was found that the addition of surfactant to both colloidal dispersions and standards led to measured metal contents close to the theoretical value. Most reliable results were obtained using the anionic surfactant sodium dodecyl sulfate (SDS) in concentrations above the critical micelle concentration. Physicochemical properties of both metal standards and colloidal dispersions were investigated to explain the apparent metal values detected. The results presented here were published as Accurate elemental analysis of metal-containing polymer latexes using inductively coupled plasma - optical emission spectrometry. ${ }^{[174]}$ 


\subsubsection{Introduction}

An increasing number of applications make use of functional nanocomposite particles. Such hybrid particles, consisting of inorganic and polymeric materials have been used for example in drug delivery (magnetite particles), ${ }^{[27,}{ }^{175]}$ cancer treatment (cis-platin), ${ }^{[25]}$ cell labeling (lanthanide complexes or quantum dots) ${ }^{[165,176]}$ and lithographic applications. ${ }^{[121]}$

Despite the variety of applications, the determination of the actual metal content in these hybrid nanoparticles has rarely been reported. ${ }^{[26,165]}$ The content of inorganic nanoparticles in the nanocomposites has mainly been determined with TGA or, in the case of magnetic particles with magnetic measurements. However, these methods lack precision, especially when lower inorganic contents are applied.

To quantify the composition of materials, inductively coupled plasma based spectrometric methods ${ }^{[154,160]}$ such as mass spectrometry (ICP-MS) or optical emission spectrometry (ICPOES) are very powerful tools and are widely used for the quality control in different industrial sectors. Traditionally, ICP methods are optimized for use the detection of metal ions in aqueous solutions. ${ }^{[158]}$ A number of reports on the use of ICP for the characterization of colloidal systems can be found in literature, mostly for the analysis of sediments on environmental sciences. ${ }^{[155-157]}$ Investigations on polymer-based hybrid nanoparticles are rare in literature. However, Vancaeyzeele et al. used ICP-MS as well as ICP-OES for the determination of lanthanide complexes in latex particles but reported lanthanide contents systemically lower than the value expected without finding an explanation for this feature of data. ${ }^{[165]}$

In this chapter, miniemulsion polymerization was applied to synthesize polymeric nanoparticles with a defined amount of metal-complex incorporated and ICP-OES was used for the determination of the metal content. In the scope of finding reliable measurement conditions, it was realized that plain measurements of pure colloidal dispersions generally gave rise to apparent metal contents significantly below the expected, theoretical value; in agreement with the findings reported by Vancaeyzeele et al. ${ }^{[165]}$ In order to examine the behavior of colloidal dispersions in the ICP spectrometer, a systematic study on measurement conditions was performed including variations of device parameters and different sample preparation strategies. The latter variations included variations in concentration of colloids, change of polymeric material as well as addition of surfactants and electrolytes. The results were correlated to the physicochemical properties of the colloidal particles and dispersions, such as hydrodynamic diameter, $\zeta$-potential and surface tension. 


\subsubsection{Results and Discussion}

\subsection{Synthesis of standard polymer particles}

Miniemulsion polymerization was applied to prepare polymer nanoparticles with defined amounts of metal complexes encapsulated.

Only commercially available metal complexes were used for encapsulation, namely ßdiketonato complexes of platinum, iron and europium. This choice covers all different groups of transition metals ranging from $3 \mathrm{~d}$ over $5 \mathrm{~d}$ elements to a member of the lanthanide group. SEM images of the prepared particles are shown in Figure 5.1.1.

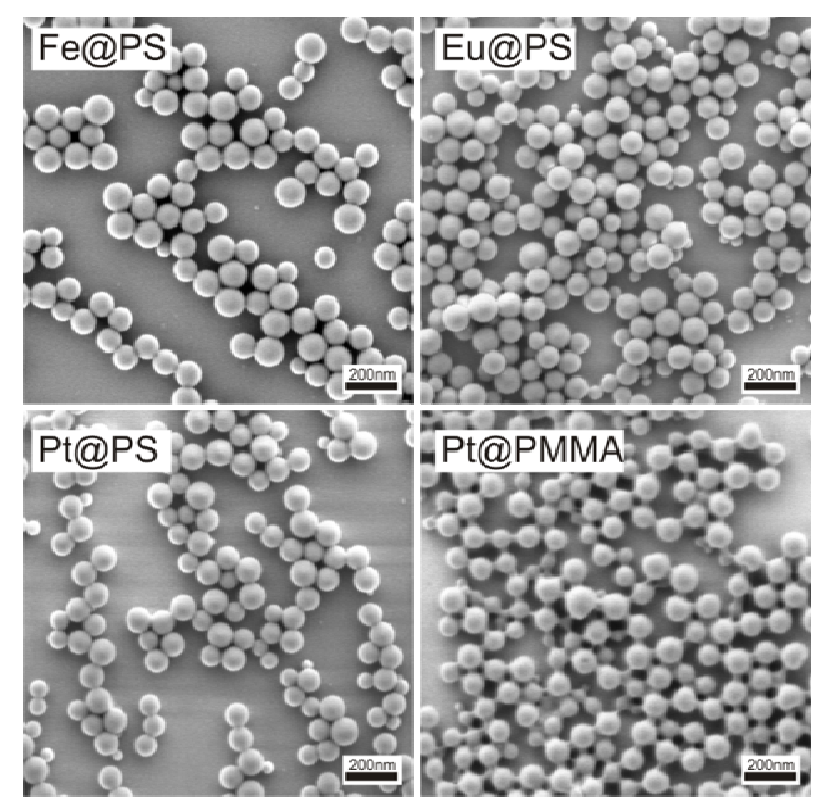

Figure 5.1.1. SEM images of the metal complex containing colloidal particles.

Table 5.1.1 summarizes the reaction parameters as well as important characteristics of the resulting particles investigated in this study. The particles of all dispersions featured sizes around $100-150 \mathrm{~nm}$ as determined by DLS and SEM and a solid content that was close to the theoretical value of $21.2 \%$, indicating high conversions. As no coagulum was found in the miniemulsions and no further treatment that could possibly remove complex or polymer from the dispersion as centrifugation or dialysis of the dispersions was performed, it is valid to assume that the total amount of metal complex present in the dispersion is equal to the amount added to the monomer phase. Thus, a theoretical metal concentration can be calculated that, within the experimental errors, is expected to be found in ICP-OES analysis of the colloid 
dispersions and will hereafter be addressed as $100 \%$ metal content. In one of the samples, Eu@PS, a minor amount of coagulum was found after filtration. In that case, small deviations from the theoretical metal content were expected.

The experimental errors mainly consist of inaccuracies during determination of solid contents, weighing of both colloidal dispersion and standards and add up to the intrinsic error given by the ICP measurement. They can be expected to be in the order of 1-3\%. For further comparison, two different polymers, polystyrene (PS) and poly(methylmethacrylate) (PMMA) were used for the encapsulation of equal amounts of $\operatorname{Pt}(\mathrm{acac})_{2}$. The polymers were chosen as they represent the most commonly used polymers in colloidal sciences and thus, are of prime interest for a detailed elaboration of an ICP-OES measurement protocol.

Table 5.1.1 Characterization of the standard particle prepared by miniemulsion polymerization. All particles contained $1.0 \mathrm{wt} .-\%$ of Metal complex with respect to the monomer.

\begin{tabular}{|c|c|c|c|c|c|c|}
\hline Sample & Polymer & $\begin{array}{c}\text { Me- } \\
\text { Complex } \\
\text { (amount) }\end{array}$ & $\begin{array}{l}\text { Metal- } \\
\text { content, } \\
\text { Theory } \\
\text { [wt.-\%] }\end{array}$ & $\begin{array}{c}\text { Solid } \\
\text { Content } \\
\text { [\%] }\end{array}$ & $\begin{array}{c}\text { Hydrodynamic } \\
\text { Diameter } \\
{[\mathrm{nm}]}\end{array}$ & $\begin{array}{c}\text { Diameter } \\
\text { SEM } \\
\text { [nm] }\end{array}$ \\
\hline Fe@PS & PS & $\begin{array}{c}\mathrm{Fe}(\mathrm{acac})_{3} \\
(60 \mathrm{mg})\end{array}$ & 0.149 & 17.4 & 148 & $103 \pm 12$ \\
\hline Eu@PS & PS & $\begin{array}{c}\mathrm{Eu}(\mathrm{tmhd})_{3} \\
(60 \mathrm{mg})\end{array}$ & 0.217 & 19.5 & 114 & $96 \pm 14$ \\
\hline Pt@PS & PS & $\begin{array}{c}\operatorname{Pt}(\mathrm{acac})_{2} \\
(60 \mathrm{mg})\end{array}$ & 0.467 & 18.4 & 107 & $97 \pm 12$ \\
\hline Pt@PMMA & PMMA & $\begin{array}{c}\operatorname{Pt}(\mathrm{acac})_{2} \\
(60 \mathrm{mg})\end{array}$ & 0.467 & 19.3 & 136 & $92 \pm 11$ \\
\hline
\end{tabular}

\subsection{Standard ICP measurements}

As a first series of measurements, the polystyrene dispersion containing $1 \mathrm{wt} .-\%$ of $\operatorname{Pt}(\mathrm{acac})_{2}$ (Pt@PS) was diluted with water to produce dispersions with solid contents ranging from 0.01 wt.-\% to 1 wt.- $\%$. Figure 5.1.2 shows the platinum contents as measured by ICP-OES. For reasons of clarity, all data depicted in this article show the relative metal content given in percentage of the theoretical value [100\%].

The platinum contents determined by the ICP-OES measurements (Figure 5.1.2) only reached values of 50 to $70 \%$ of the theory. The more concentrated dispersions gave higher contents compared to the less concentrated ones. Additionally, the values measured were difficult to 
reproduce and varied between 50 and $70 \%$, as expressed by the large error bars. As the more concentrated dispersions ( 0.5 and 1 wt.- $\%)$ tended to occasionally clog the nebulizer, 0.1 wt.-\% dispersions were considered to give a compromise between sufficient total amount of platinum in the dispersion and ease of handling as no clogging occurred during measurements. Therefore, this concentration was used for all experiments conducted afterwards.
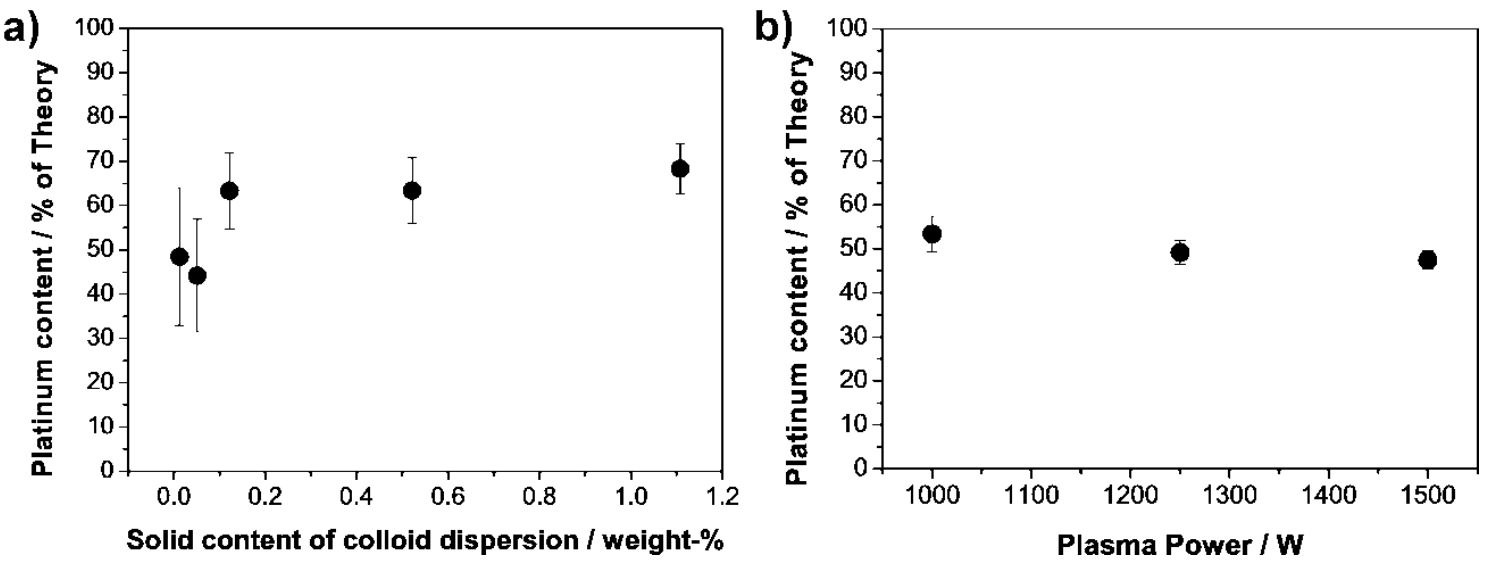

Figure 5.1.2. a) Effect of particle concentration on apparent Pt content by ICP-OES. b) Variation of plasma power and effect on the platinum values detected exemplarily shown for the sample Pt@PS.

As a possible explanation for the low metal content detected, it was hypothesized that the combustion of organic matter additionally present in the dispersion might withdraws energy from the plasma being no longer available for metal-ion excitation. ${ }^{[158,177]}$ Thus, lower values for the metal content are measured. The hypothesis was tested with two experiments. First, the plasma power was systematically increased from 1000 to $1500 \mathrm{~W}$. Figure 5.1.2b shows the measured platinum concentration for a $0.1 \mathrm{wt} .-\%$ dispersion of colloids. As can be seen, the variation of plasma power did not significantly increase the detected amount of platinum. On the contrary, a minor shift of about $4 \%$ to lower values was observed for higher plasma power. A higher plasma power affects the colloid combustion and by this, the efficiency of ionization of the platinum atoms within the particles. Therefore, it was expected that if combustion of the organic material of the colloidal particles interferes with metal atom excitation, variation of plasma power would lead to differences in the detected metal content. However, the metal content detected was essentially the same with varying plasma power. Hence, it is concluded that differences in excitation efficiency of the metal inside the particles caused by organic matter present in the plasma as compared to the free metal ions in the standards is not the cause of the low metal contents detected by ICP-OES measurements.

As a second experiment, pure polystyrene colloids made by a similar recipe without platinum complex were added to the platinum standard solution. Doing so, the organic contents in both standard solutions and colloid dispersion were matched and any difference in excitation 
efficiency by combustion was consequently taken into account during the calibration with standards.

Table 5.1.2 shows the results for the measurements with and without the addition of colloids to the standard solution for Pt-containing PS and PMMA latex particles. For both polymer types, no changes of the measured values were detected. Hence, any effect of additional organic matter for the low metal values detected was excluded.

Table 5.1.2. Measurement results with and without the addition of 0.1 wt.- $\%$ of pure PS colloids to PtStandards, $1250 \mathrm{~W}$ plasma power.

\begin{tabular}{cccc}
\hline Sample & $\begin{array}{c}\text { PS-colloids in } \\
\text { Standard } \\
\text { [wt.-\%] }\end{array}$ & Pt-content & $\begin{array}{c}\text { ICP standard- } \\
\text { deviation }\end{array}$ \\
\hline [\% of Theory] & {$[\%]$} \\
Pt@PS & No & 49.16 & 5.41 \\
Pt@PMMA & Yes $(0.1)$ & 51.85 & 6.34 \\
Pt@PMMA & No & 73.87 & 3.05 \\
\hline
\end{tabular}

\subsection{Size issues}

As effects of additional organic matter in the plasma for the unexpected metal contents detected were excluded, it was concluded that the problem might be found in the behavior of colloidal matter as samples fed in the ICP-OES device. It was assumed that due to their size and mass, colloidal particles might show a different behavior in the sample inlet system than free ions in solution. Especially in the cyclone chamber, a difference between plain ions and comparably large and heavy colloidal particles might lead to a discrepancy in acceleration of the colloidal particle dispersion into the chamber. Hence, a lower amount of metal-containing particles would arrive in the plasma, be combusted, excited and finally detected compared to an equally concentrated solution of free metal ions.

To gain insight into the behavior of differently sized colloids in the cyclone chamber and the sample inlet system, a seeded emulsion polymerization (see chapter 5.1.2.5) was performed. Seed particles containing $\operatorname{Pt}(\mathrm{acac})_{2}$ were consecutively increased in size by adding further polystyrene to the colloidal particles without altering the total amount of $\mathrm{Pt}(\mathrm{acac})_{2}$ per particle. Particles with size of $164 \mathrm{~nm}$ (seed), $250 \mathrm{~nm}, 427 \mathrm{~nm}$ and $527 \mathrm{~nm}$ were produced and analyzed with the ICP-OE spectrometer. $\mathrm{As} \mathrm{Pt}(\mathrm{acac})_{2}$ is hydrophobic, no complex molecules can diffuse out of the seed particles during the seeded polymerization reaction. Hence, each particle produced contained a similar amount of platinum, regardless of its size. With an increase in total 
mass of the colloids, the ratio between inorganic and organic material decreased, as the amount of platinum complex did not change but more polymeric, organic material was added to the colloids. Table 5.1.3 summarizes important properties of the seed particles.

Table 5.1.3. Properties of the colloidal particles prepared by seed polymerization.

\begin{tabular}{ccccc}
\hline Diameter & Mass & $\begin{array}{c}\text { Pt content } \\
\text { (Theory) }\end{array}$ & $\begin{array}{c}\text { Pt-content } \\
\text { (Theory) } \\
\text { [wt.-\%] }\end{array}$ & $\begin{array}{c}\text { Pt-content } \\
\text { (Theory) } \\
\text { [\% \% of seed] }\end{array}$ \\
\hline $\mathbf{1 6 4}$ (seed) & 100 & 34 & 0.450 & 100 \\
$\mathbf{2 5 0}$ & 348 & 34 & 0.129 & 28.8 \\
$\mathbf{4 2 7}$ & 1733 & 34 & 0.026 & 5.8 \\
$\mathbf{5 2 7}$ & 3258 & 34 & 0.014 & 3.1 \\
\hline
\end{tabular}

In order to evaluate the effect of particle sizes and masses, the Pt content determined by the ICP measurement for the seed particle was arbitrarily set to 1.00 . The relative detected platinumcontents are shown in Figure 5.1.3. The contents measured for the seeded particles with bigger diameters were in the same order of magnitude and showed values between 1.00 and 1.20 , without a visible trend. No decrease in platinum content with respect to the seed particles was detected. Note that the increase in measurement error for bigger particles stems from the fact that all samples were measured as $0.1 \mathrm{wt} .-\%$ dispersions. The total number of particles present in the 0.1 wt.- $\%$ dispersion is not equal for all different particle sizes, as the mass of a single particle increases strongly with increase in diameter. Therefore, even though the platinum amount per particle remains the same, the total amount of platinum present in the dispersion decreases with increasing particle diameter. A lower total amount of platinum atoms to be detected in the sample induces higher relative errors during measurement.

Additionally, the set of experiments showed that an increase in organic material relative to the metal does not alter the ICP results and hence, support the conclusions drawn from the standard measurements (see Table 5.1.2).

Summarizing these experiments, it can be stated that the size of the colloidal particles does not affect the ICP measurements, at least in the size range investigated. 


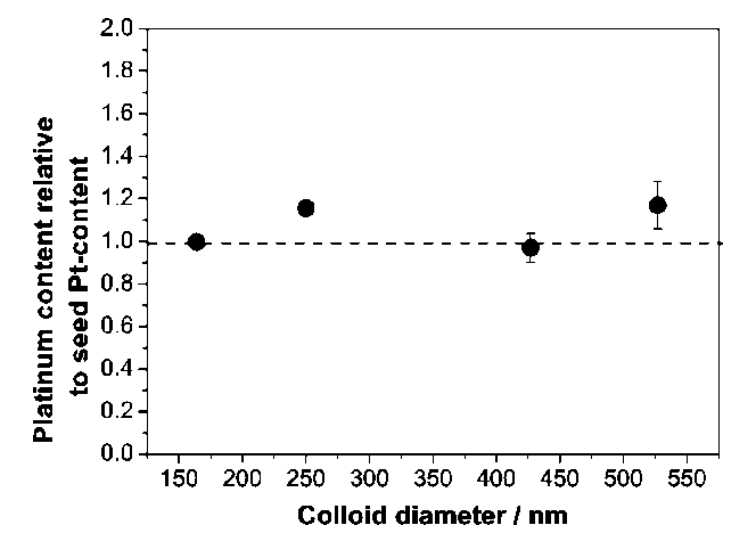

Figure 5.1.3. Relative amount of platinum determined for colloids with the same amount of $\operatorname{Pt}(\mathrm{acac})_{2}$ but different diameters synthesized by a seeded polymerization. The platinum content of the seed particles was arbitrarily set to 1.0 for reasons of clarity.

\subsection{Surfactant addition}

In the following set of experiments, the addition of surfactants to metal containing latexes and metal standard solutions was examined. Several properties, such as interfacial tensions and the particle surface properties of the colloidal dispersion and metal standard solutions change upon the addition of surfactants.

Miniemulsion particles are known to have an incomplete surface coverage with surfactant molecules. ${ }^{[18]}$ Therefore, an increase in surfactant concentration induces a higher degree of stability for the colloidal particles as the added surfactant molecules will assemble at the interface between water and particles. Depending on the chemical nature of the surfactant, an increase in stability against coagulation is induced either electrostatically by ionic surfactants or sterically by nonionic surfactants. Furthermore, as the particles become more effectively stabilized, additional surfactant might prevent the particles from interacting with surfaces in the sample feeding system.

The addition of surfactant will generally lower the surface tension of both colloid dispersion and metal standard solution to a saturation value, reached at the critical micelle concentration $(\mathrm{cmc})$. Changes in surface tension can lead to different behavior of the liquid during transport and nebulization and hence, can affect the input of analyte into the plasma. ${ }^{[178]}$ Additionally, it has been shown for inorganic particles that a high degree of stability is of crucial importance for reliable ICP-OES characterization using slurry methods. ${ }^{[164]}$

Two different surfactants, ionic sodium dodecyl sulfate (SDS) and nonionic Triton X100 were chosen. First, ICP measurements of all different dispersions were performed with varying 
amounts of surfactant. The results were evaluated against metal standards that did not contain any surfactant (Figure 5.1.4). Care was taken to choose elemental emission lines that did not show any spectral interference with sodium lines or lines originating from contaminants (e.g. $\mathrm{Mg}$ from SDS) and thus, guarantee spectrally reliable information of the metal content.

The left side of Figure 5.1.4 (plots A-D) shows the results of the ICP-OES analysis for samples with SDS amounts between 0 and 1.5 wt.- $\%$. The behavior of all different colloidal dispersions was strikingly similar: the addition of small amounts of SDS leads to a drastic increase in the metal content measured. With increasing SDS concentration, the detected values continuously rose until they reached a plateau, starting from an SDS concentration of $0.5 \mathrm{wt} .-\%$, of constant values very close to the theoretical amount.
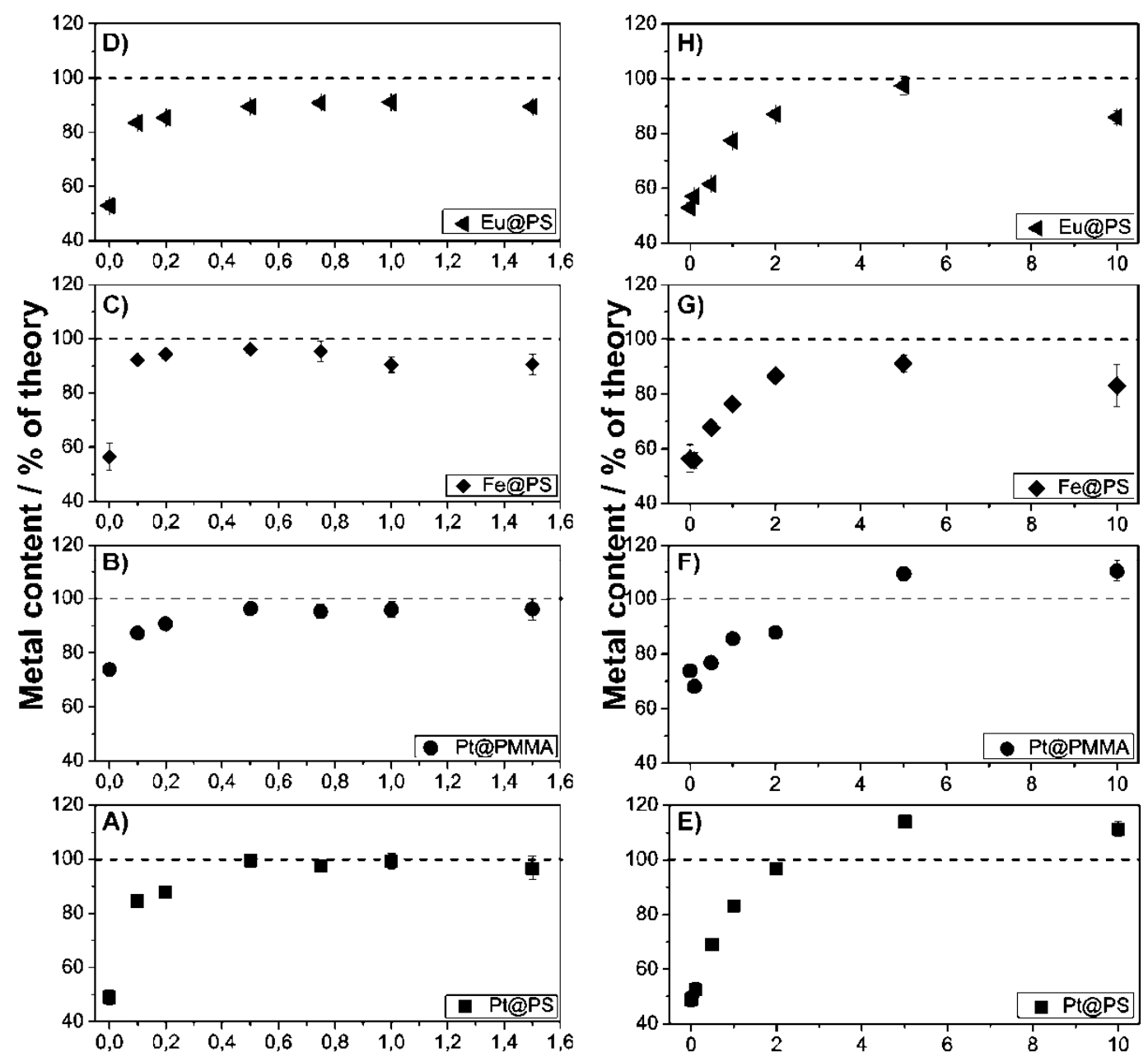

SDS content / wt- $\%$ of $\mathrm{H}_{2} \mathrm{O}$

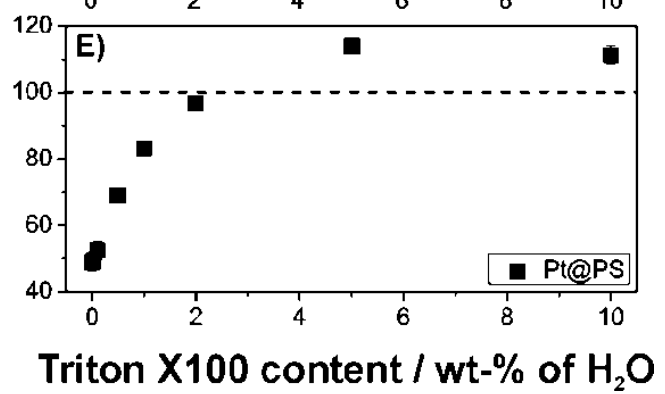

Figure 5.1.4. Effect of surfactant addition on metal content measured by ICP-OES. Left side (A-D): measured metal contents after addition of SDS to $0.1 \mathrm{wt} .-\%$ colloid dispersions; right side (E-H): measured metal contents after addition of Triton X100 to

0.1 wt.- $\%$ colloid dispersions. The dotted line represents the theoretical metal content. 
Quantitatively, minor differences between the different samples exist. First, the sample containing europium (Eu@PS) only exhibited a Eu content of approximately 92\% of the theoretical value when measured with SDS concentrations of 0.5 and 0.75 wt.- $\%$ while all other samples showed values of $96 \%$ or higher. This is attributed to a small amount of coagulum present in the europium containing emulsion after polymerization. Second, apparent metal contents measured with the highest SDS concentrations of $1.5 \mathrm{wt} . \mathrm{-} \%$ decreased to $90 \%$ of the theory for the Eu- and Fe containing samples Eu@PS and Fe@PS whereas measurements of the platinum containing samples (Pt@PS and Pt@PMMA) still featured apparent metal contents of more than $96 \%$.

However, metal contents very close to the theoretical value were measured reproducibly for SDS amounts between 0.5 and $1 \mathrm{wt} .-\%$ for all samples. The fact that the theoretical value was not exceeded with increasing SDS concentration indicates that the lines chosen for the analysis indeed did not show any spectral interference with other elements, especially $\mathrm{Na}$.

The right side of Figure 5.1.4 (plots E-H) depicts the results of the ICP-OES measurements obtained for metal containing colloid dispersions with the surfactant Triton X100 added. Qualitatively, a similar behavior as for the SDS addition was found. With increasing Triton X100 concentration, the metal concentrations detected significantly increased. However, there are two differences: First, the concentrations of Triton X100 necessary to induce significant increases in metal content detected are higher compared to SDS. Second, the Triton X100 concentrations that gave rise to values close to $100 \%$ vary from $2 \mathrm{wt} .-\%$ for platinum containing dispersions to 5 wt.-\% for the sample containing europium. For the Fe@PS sample, a maximum $\mathrm{Fe}$ concentration of $91 \%$ of theory was measured with $5 \mathrm{wt} . \mathrm{\%}$ Triton X100. Finally, for the platinum containing particle dispersions, the Pt content detected at high concentrations exceeded the theoretical value by about $10 \%$.

Although the colloidal dispersions were measured with additional surfactant, no surfactant was added to the standard solutions. Therefore, the surface tension between colloidal samples and metal standards differed drastically. This can lead to a different behavior of the liquids during the transport through the spectrometer and in the nebulizer chamber and may lead to differences in the apparent metal concentration detected. Additionally, the presence of sodium ions in a sample can lead to disturbances of the measurements known as matrix effects. Matrix effects have their origin in different parts of the ICP spectrometer, ranging from differences in excitations in the plasma itself to changes in the solvent transportation rate in the sample feeding system. ${ }^{[158,179-180]}$

In order to avoid these differences and to come up with a more reliable measurement protocol, similar concentrations of surfactant were used for both standards and colloid dispersion. This procedure, commonly referred to as matrix adaption, guarantees maximum similarity between 
standards and samples and hence, gives rise to more trustworthy results of the ICP-OE spectrometer. $^{[158,180]}$
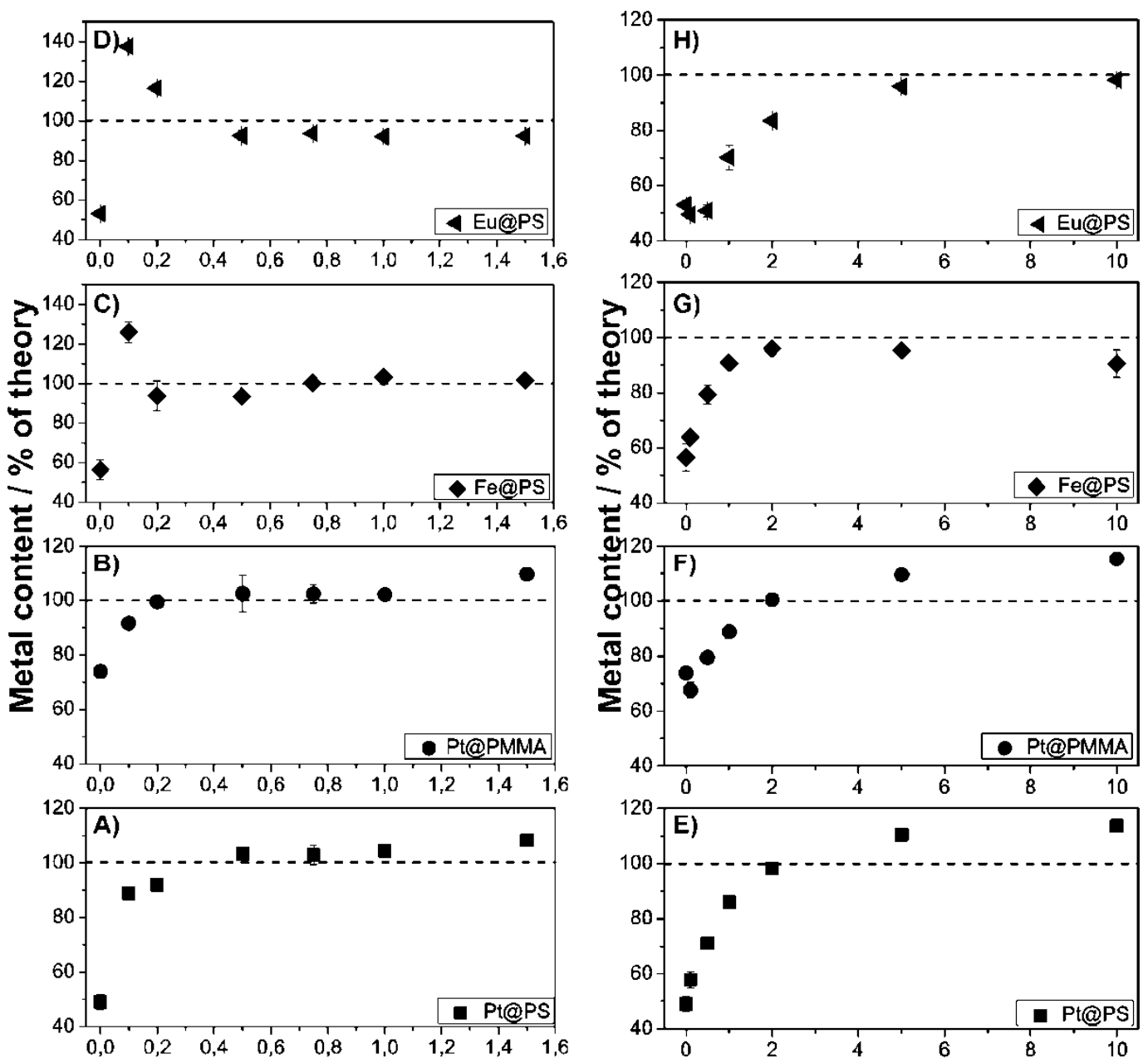

SDS content / wt- $\%$ of $\mathrm{H}_{2} \mathrm{O}$

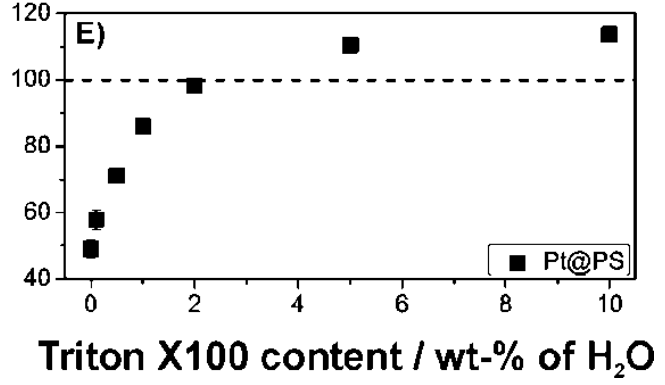

Figure 5.1.5. ICP-OES results for samples measured after addition of surfactants to both colloid dispersion and metal standard solutions (matrix adaption). Left side (A-D): SDS as surfactant; right side

(E-H): Triton X100 as surfactant. The dotted line represents the theoretical metal content.

Using matrix adaption, all standards were matched with the surfactant concentrations used for the samples. The resulting values for the metal concentration are shown in Figure 5.1.5.

Generally, the individual plots do not drastically differ from the measurements performed without matrix adaption (Figure 5.1.4) but, as stated before, can be considered to be more accurate. Plots A-D (left side of Figure 5.1.5) show the dependence of SDS on the platinum values measured. Most importantly, the plateau of constant values close to the theoretical metal content was still found for SDS concentrations between 0.5 and 1 wt.-\%. Quantitatively, the metal values were even closer to the theoretical value and a maximal difference of $7 \%$ compared to the theoretical value was measured for all samples investigated. The apparent 
platinum concentrations detected for both Pt@PS and Pt@PMMA were 102-103\% of the theoretical value, the iron containing sample gave rise to iron contents of $100-103 \%$ of the theoretical value and the europium containing sample showed a metal content of $92-94 \%$ of theory. As mentioned above, this discrepancy between the measured and the theoretical europium content is attributed to the presence of a minor amount of coagulum found after synthesis. For all other samples, the metal contents measured matched the theoretical value within the experimental accuracy.

The most prominent difference between measurements with and without matrix adaptions are the values obtained for small concentrations of surfactant (see Figure 5.1.5 C and D) that, for Fe@PS and Eu@PS samples, significantly exceed the theoretical value. This unexpected behavior can be explained by a difference in surface tension for the metal standards solution and will be discussed in the next section.

The right side of Figure 5.1.5 shows the results for matrix-adapted measurements with Triton X100 as surfactant. Qualitatively, the measured metal concentrations show a very similar dependence compared to measurements with plain standard solutions (Figure 5.1.4 E to H).

A quantitative analysis reveals values closer to the theoretical value for higher Triton X100 contents for the matrix adapted measurements. Using 2 wt.- $\%$ of Triton X100, the metal contents measured were within $4 \%$ difference to the theoretical value for all samples except for Eu@PS. For the latter, the metal values closest to theory were obtained with 5- and 10 wt.-\% of Triton X100 (97 and 98\% respectively).

Concluding this section, it can be stated that addition of surfactant drastically influences the results of the ICP-OES measurements. Choosing proper concentrations of the surfactant, apparent metal values in very good agreement with the theoretical value were obtained. These values were determined to be between 0.5 and $1 \mathrm{wt} . \%$ for SDS as surfactant and approx. $2 \mathrm{wt.}-\%$ for Triton X100. Using matrix adaption, systematic errors caused by differences between standard solution and colloid dispersion were minimized.

Furthermore, no influence of the material of the polymeric shell was detected as the samples Pt@PS and Pt@PMMA showed very similar behavior during all different measurements.

\subsection{Surface tension}

In order to further investigate the characteristics of the colloidal dispersions with various concentrations of surfactant, surface tension measurements were performed using a Du Noüy ring tensiometer. Figure 5.1.6 shows surface tensions obtained for dispersions of platinum containing polystyrene dispersions with a solid content of $0.1 \mathrm{wt} . \mathrm{\%} \%$ that were used for the ICP- 
OES analysis described in the previous chapter. For comparison, surface tensions of equal amounts of SDS dissolved in pure water were measured as well.
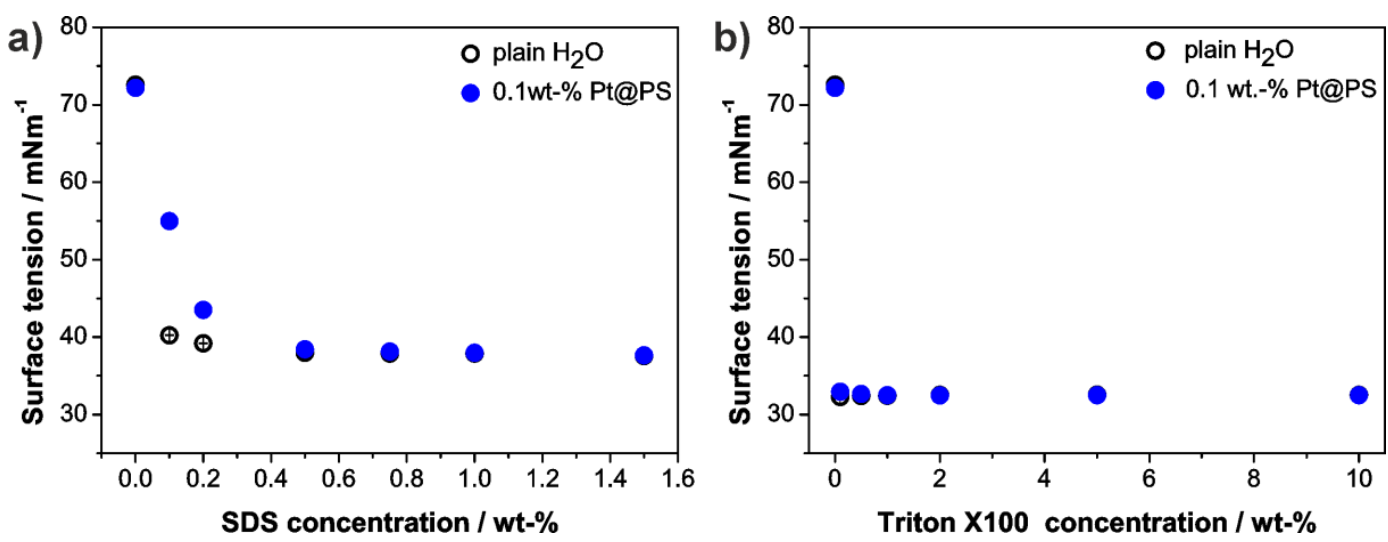

Figure 5.1.6. Surface tension measurements of the colloidal dispersions and pure water with varying amounts of surfactants. a) SDS used as surfactant. b) Triton X100 used as surfactant.

All measurements were performed with the colloidal dispersions of $0.1 \mathrm{wt} .-\%$ used to measure ICP-OES. Figure 5.1.6a compares the surface tension of water with varying amounts of SDS with those of the colloidal dispersion Pt@PS. As expected, both sets of measurements showed a drastic decrease in surface tension with increasing amounts of surfactant until a saturation is reached upon which further addition of SDS did not alter the surface tension anymore. The onset of this plateau corresponds to the critical micelle concentration $(\mathrm{cmc})$ of the surfactant in the respective medium. While the colloidal dispersion and pure water qualitatively resemble each other, quantitatively, they do show differences. Most prominent, the surface tension values for small SDS concentrations $(<0.5 \%)$ are significantly higher for the colloidal dispersion, a characteristic that can be attributed to surfactant molecules being adsorbed to the surface of the colloidal particles. These molecules are thus not present at the liquid's surface to reduce the surface tension. A further consequence is the cmc of SDS in dispersion, which is shifted to a higher SDS concentration compared to pure water containing SDS. Finally, the first data point measured for the colloidal dispersion that is situated at the plateau of constant values representing surfactant concentrations above the cmc is obtained for the dispersion with 0.5 wt.- $\%$ SDS. Strikingly, this concentration was shown to be the minimum concentration of surfactant to give experimental metal contents resembling the theoretical value for all samples investigated (Figure 5.1.5). Hence, it is concluded that, in the case of SDS as surfactant, the cmc represents a threshold value of surfactant concentration for reliable measurements of the metal content in colloidal dispersions. This behavior is attributed to the fact that starting with the $\mathrm{cmc}$, the particles' surfaces are completely saturated with surfactant molecules and hence, interactions with other particles and surfaces in the spectrometer are reduced to a minimum. 
Additionally, above the cmc, the surface tension is at the lowest possible point for both colloidal dispersions and water standards. This leads to a similar behavior of both types of liquids in the nebulizer chamber and therefore, to comparable input of matter into the plasma that finally enables reliable ICP-OES measurements.

For Triton X100 as surfactant, no differences between pure water and the colloid dispersion are visible as even for the lowest Triton X100 concentration ( $0.1 \mathrm{wt} .-\%)$, as the cmc has a value of 0.015 wt.- $\%$ as specified by the manufacturer. The surface tension directly drops to stable values of $32 \mathrm{mN} \cdot \mathrm{m}^{-1}$ for the lowest concentration measured and remains constant for higher concentrations of surfactant. It can be concluded that the high Triton X100 concentrations necessary for measurements with detected values close to theory cannot be related to the $\mathrm{cmc}$ of Triton X100.

Finally, surface tension measurements were performed to investigate the behavior of colloidal dispersions and metal standard solutions with low SDS amounts of 0.1 wt.- $\%$ that gave rise to unexpected results (see Figure 5.1.5A-D) for the apparent metal content. Platinum containing samples gave similar values for measurements with and without matrix adaption (around $80 \%$ of the theoretical value). Iron and europium containing samples on the contrary, lead to apparent metal contents significantly higher than the theoretical value (above 130\%) when measured with matrix adaption while measurements with plain standards produced values around $80-90 \%$ of the theoretical value.

To resolve this unexpected phenomenon, two experiments were performed. First, the surface tensions of the metal-ion solutions containing $0.1 \mathrm{wt.} . \%$ SDS used as standards for the calibration of the ICP spectrometer were measured (Figure 5.1.7b).

Surprisingly, the surface tension of both europium and iron solutions dropped rapidly with increasing amount of metal ions from $45 \mathrm{mN} \cdot \mathrm{m}^{-1}$ for $0.5 \mathrm{mg} \cdot \mathrm{l}^{-1}$ 1 solutions to $32 \mathrm{mN} \cdot \mathrm{m}^{-1}$ for $2 \mathrm{mg} \cdot \mathrm{l}^{-1}$ solutions. The platinum standard solutions did show surface activity as well, but the decrease was less pronounced with a shift from 45 to $40 \mathrm{mN} \cdot \mathrm{m}^{-1}$ between lowest and highest metal concentration.

For comparison, europium standards with $0.5 \mathrm{wt.}$ - $\%$ SDS were investigated as well. As can be seen in Figure 5.1.7b, no changes in surface tension for the different metal concentrations were detected. As the SDS concentration is well above cmc, no further changes are induced by the metal ions. This behavior once again reflects the reliable metal contents detected by ICP for SDS concentrations of $0.5 \mathrm{wt} .-\%$ or higher.

The influence of varying surface tensions of the different standard solutions for an ICP-OES measurement was investigated consecutively. A standard solution containing $1 \mathrm{mg} \cdot \mathrm{l}^{-1}$ metal ions was prepared and analyzed in the spectrometer. As standards for the calibration line, the 
standard solutions of the matrix adapted measurements were used. These contained varying amounts of SDS. Figure 5.1.7a shows the results of the ICP measurements.
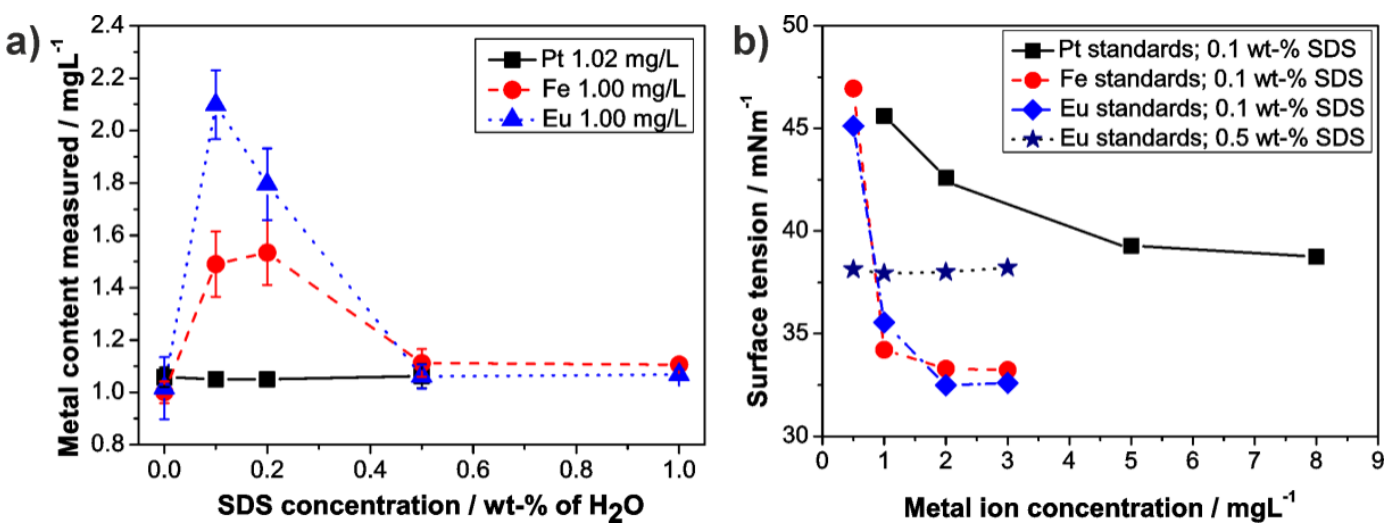

Figure 5.1.7. Surface tension and ICP performance of the metal standard solutions. a) surface tension measurements of the metal standards used for the matrix adapted measurements with $0.1 \mathrm{wt} .-\%$ of SDS. For comparison, europium standards containing $0.5 \mathrm{wt} .-\%$ SDS are shown as well ( $\star)$. b) ICP-OES test measurements to reveal problems arising from changing surface tension: a solution containing $1 \mathrm{mg} \cdot \mathrm{l}^{-1}$ metal ions was measured with standards containing different amount of SDS. The lines interconnecting the data points are guides for the eyes only.

For the platinum sample, concentrations close to the expected value of $1.02 \mathrm{mg} \cdot 1^{-1}$ were found for all SDS concentrations added to the standards. The europium and iron sample, prepared to contain $1.00 \mathrm{mg} \cdot 1^{-1}$ metal ions as well, showed a very different behavior. ICP-OES measurements with plain aqueous standards gave apparent metal contents of $1.00 \mathrm{mg} \cdot \mathrm{l}^{-1}$, as expected. However, when adding low concentrations of SDS to the calibration standards, the apparent metal content given by the spectrometer greatly exceeded the true metal content and indicated metal contents of $1.5 \mathrm{mg} \cdot \mathrm{l}^{-1}(\mathrm{Fe})$ and $2.1 \mathrm{mg} \cdot \mathrm{l}^{-1}(\mathrm{Eu})$ respectively. As soon as the SDS concentration in the standards exceeded the $\mathrm{cmc}$ (SDS $\geq 0.5 \mathrm{wt} .-\%$ ), the metal contents detected were close to the true concentrations. Again, this corroborates the results given before that for reliable measurements using SDS as surfactant, concentrations well above the cme have to be chosen.

Comparing the apparent metal content detected for small SDS concentrations for Pt and Eu and $\mathrm{Fe}$ with the surface tensions of the respective standards used, it is concluded that large differences in surface tension within the standards explains the overestimation of the metal contents measured for the iron and europium solution. Additionally, we believe the same phenomenon accounts for the overestimation of $\mathrm{Fe}$ - and Eu-containing colloidal dispersions with low SDS contents presented in Figure 5.1.5 C and D. 


\subsection{Effect of electrolyte addition}

To examine the differences between SDS and Triton X100 addition for the apparent metal detection, experiments with different ionic strength of both dispersions and standards were performed. SDS, being an anionic surfactant alters the ionic strengths in the colloidal solutions as it represents a $[1 ; 1]$ electrolyte while Triton X100, being nonionic, does not induce any changes in the ionic strengths. Even though reliable measurements using Triton X100 in high concentrations of $2 \mathrm{wt} .-\%$ or more can be performed, the addition of SDS seems to be more effective as only concentrations of $0.5 \mathrm{wt} . \mathrm{-} \%$ are necessary to achieve metal contents resembling the theoretical value.

To clarify the effect of electrolyte addition to the colloid dispersion, different electrolytes were added to dispersions of 0.1 wt.-\% of Pt@PS and Fe@PS. Table 5.1.4 sums up the dispersions prepared and the resulting metal contents measured by ICP-OES.

First, sodium chloride $(\mathrm{NaCl})$ was chosen as electrolyte as it contains sodium ions as well but does not show any surface activity. The concentration was set to $25.67 \mathrm{mmol} \cdot 1^{-1}$, a value that is equimolar to $0.75 \mathrm{wt} . \mathrm{\%} \%$ of SDS, the concentration applied to obtain good agreements with the theoretical metal contents in previous experiments. Surprisingly, the addition of $\mathrm{NaCl}$ to the colloidal dispersion had similar effects as the addition of SDS: the metal content measured increased from around $50 \%$ of the theoretical value for plain solutions to values close to $100 \%$ for solutions containing $\mathrm{NaCl}$ for both iron and platinum ions encapsulated.

Table 5.1.4. Effect of electrolyte addition on ICP results; all standards with equivalent amounts of electrolytes; 0.1 wt.- $\%$ dispersions of polystyrene.

\begin{tabular}{|c|c|c|c|c|c|}
\hline Sample & Electrolyte & $\begin{array}{c}\text { Conc. } \\
{\left[\mathrm{mmol} \cdot \mathrm{I}^{-1}\right]}\end{array}$ & $\begin{array}{c}\text { Ionic strength } \\
{\left[\mathrm{mmol} \cdot \mathrm{I}^{-1}\right]}\end{array}$ & $\begin{array}{l}\text { Me-content } \\
\text { [\% of Theory] }\end{array}$ & $\begin{array}{c}\text { ICP standard } \\
\text { deviation } \\
{[\%]}\end{array}$ \\
\hline $\begin{array}{l}\mathbf{P t} @ P S \\
\end{array}$ & --- & 0 & 0 & 49.16 & 5.41 \\
\hline Pt@PS & $\mathrm{NaCl}$ & 25.67 & 25.67 & 97.33 & 3.52 \\
\hline Pt@PS & $\mathrm{KCl}$ & 25.67 & 25.67 & 103.65 & 6.58 \\
\hline Pt@PS & $\mathrm{CaCl}_{2}$ & 8.56 & 25.67 & 104.03 & 2.71 \\
\hline Fe@PS & --- & 0 & 0 & 56.39 & 9.96 \\
\hline Fe@PS & $\mathrm{NaCl}$ & 25.67 & 25.67 & 100.56 & 5.48 \\
\hline Fe@PS & $\mathrm{KCl}$ & 25.67 & 25.67 & 101.79 & 4.11 \\
\hline Fe@PS & $\mathrm{CaCl}_{2}$ & 8.56 & 25.67 & 93.95 & 5.03 \\
\hline
\end{tabular}

In order to exclude effects of sodium on the measured values, potassium chloride $(\mathrm{KCl})$ was chosen as another $[1 ; 1]$ electrolyte in similar molarity. Therefore, the colloidal dispersions had a similar ionic strength. As can be seen from Table 5.1.4, the impact on the metal contents 
determined by ICP-OES was comparable: measured values for the metal content increased significantly to the theoretical value within the limits of experimental errors for both colloidal dispersions used in the experiments.

Last, calcium chloride $\left(\mathrm{CaCl}_{2}\right)$ was chosen as a $[2 ; 1]$ electrolyte. In that case, not the molar concentration was held constant but the ionic strength of the solution. The molarity of the solution was set to $8.56 \mathrm{mmol} \cdot \mathrm{l}^{-1}$ in order to achieve an ionic strength of $25.67 \mathrm{mmol} \cdot \mathrm{l}^{-1}$. Again, the metal contents measured with this ionic strength increased drastically compared to the plain solution and deviations from the theoretical value were restricted to several percents.

As stated before, the presence of alkali- and earth alkali elements in aqueous analyte solutions can affect the amount of analyte ions detected by interferences known as matrix effects ${ }^{[158,179]}$ that originate either from changes in the plasma itself (e.g. differences in excitation efficiency $)^{[158]}$ or during transport of the analyte solution (e.g. changes in transport rates). ${ }^{[158,179-}$ ${ }^{180]}$ Matrix matching was recognized as a means to minimize these matrix effects. ${ }^{[158]}$ As all our measurements were performed with matrix-matched standards, we believe that the increase in apparent metal content detected cannot be attributed to matrix effects but needs to be attributed to changes of the colloidal properties upon electrolyte addition.

For further investigations, particle size, zeta potential and surface tension of the particle dispersions containing electrolytes were measured. Table 5.1.5 summarizes the results. No significant changes in hydrodynamic radius or surface tension of the different dispersions were detected after addition of the different electrolytes. The zeta potential shifts to higher negative values for $\mathrm{NaCl}(-72.4 \mathrm{mV})$ and $\mathrm{KCl}(-53.4 \mathrm{mV})$ compared to the pure colloidal dispersion ($42.0 \mathrm{mV})$. Addition of $\mathrm{CaCl}_{2}$ induces a decrease in zeta potential $(-29.6 \mathrm{mV})$. The origin of these shifts are attributed to a shrinkage of the electrochemical double layer upon electrolyte addition and the presence of positively charged calcium ions in the double layer that overcompensate the negative charge of SDS present at the particle surface. As the zeta potential does not change in a similar way for the different electrolyte solutions, no clear explanation of its effect for the apparent metal content can be hypothesized.

Table 5.1.5. Physico-chemical properties of dispersions of Pt@PS with different electrolytes.

\begin{tabular}{cccccc}
\hline Electrolyte & Conc. & Ionic strength & $\begin{array}{c}\text { Hydrodyn. } \\
\text { Diameter } \\
{[\mathbf{n m}]}\end{array}$ & $\begin{array}{c}\text { Zeta } \\
\text { Potential } \\
{[\mathbf{m V}]}\end{array}$ & $\begin{array}{c}\text { Surface } \\
\text { Tension } \\
{\left[\mathbf{m N}^{-\mathbf{m}^{-1}}\right]}\end{array}$ \\
\hline--- & 0 & 0 & 107 & $-42.0 \pm 1.6$ & 72.2 \\
$\mathbf{N a C l}$ & 25.67 & 25.67 & 103 & $-71.4 \pm 2.8$ & 72.15 \\
$\mathbf{K C l}$ & 25.67 & 25.67 & 109 & $-53.4 \pm 2.6$ & 72.52 \\
$\mathbf{C a C l}_{\mathbf{2}}$ & 8.56 & 25.67 & 120 & $-29.6 \pm 0.9$ & 73.14 \\
\hline
\end{tabular}


Summarizing these measurements, it is concluded that not only a decrease in surface tension induced by addition of surfactants does affect the apparent metal contents measured, but the mere presence of electrolyte already alters the behavior of colloidal dispersions in the ICP-OES device and reproducibly leads to the detection of metal contents close to the theoretical value. The origin of this behavior needs further investigation as no clear correlation between surface tension and zeta potential of the colloidal dispersions and the increase in apparent detected metal content was found. From a practical point of view however, it has to be stated that addition of electrolytes decreases the stability of colloidal dispersions and eventually leads to coagulation of the particles while addition of surfactants naturally increases their stability. For reliable and reproducible measurements, addition of surfactant is regarded as the method of choice.

\subsubsection{Conclusion}

In this chapter, it is shown that a simple measurement of colloidal metal-complex containing dispersions systematically underestimated the metal content of colloidal particles. Variation of plasma power or matching the total content of organic matter by addition of plain polystyrene colloids to the standards did not lead to an increase in apparent metal content.

Addition of surfactant to the colloidal dispersion and, to account for changes in the aqueous matrix, to the metal standard solutions as well, led to detected metal contents in good agreement with the theoretical value. It is assumed that the increase in measured metal contents can be explained by three different factors: a decrease in surface tension of the colloidal dispersion, more stability of the dispersion with colloids being extremely well separated and a decrease in interactions of the colloids with polymeric tubes and glass parts of the ICP-OE spectrometer. As surfactants, sodium dodecyl sulfate (SDS) and Triton X100 were both successfully used. SDS proved to be more efficient as stable metal contents close to the theoretical value were detected with concentrations from 0.5 to $1 \mathrm{wt} .-\%$. Surface tension measurements confirmed that the threshold for reliable metal content detection corresponds well with the critical micelle concentration $(\mathrm{cmc})$ of the surfactant in the colloidal dispersion. Triton X100 was successfully applied to reach metal contents close to the theoretical value in concentrations of $2 \mathrm{wt} . \mathrm{\%}$ or higher. 


\subsubsection{Synthesis of metal-complex containing latex particles}

This chapter describes the synthesis of metal-complex containing polymer particles by emulsion and miniemulsion polymerization. While surfactant-free emulsion polymerization produces particles with great homogeneity, the diffusion controlled mechanism presents a serious drawback for the controlled incorporation of functional molecules such as metal complexes. In contrast, the miniemulsion polymerization process prevents net diffusion and thus allows for a stoichiometric incorporation, even of two complexes simultaneously. However, the particle size distribution is relatively broad. A subsequent seeded emulsion polymerization process can be applied in order to control the final size of the functional polymer particles. All three reaction pathways have been performed for the incorporation of different metal acetylacetonate complexes. A detailed investigation on the encapsulation efficiency of a stoichiometric mixture of both complexes is conducted by ICP-OES.

The produced particles serve as precursor colloids for the preparation of metal nanoparticles arrays in a process that will be presented in chapter 5.3.

All plain polystyrene particles that are used in subsequent chapters for the assembly of colloidal monolayers were prepared by surfactant-free emulsion polymerization as described in the experimental section. Parts of this results were published in the articles "Platinum Nanoparticles from Size adjusted functional colloidal particles generated by a seeded emulsion polymerization process" ${ }^{[181]}$ and "Interfacial activity of uncharged, highly symmetric metal $\beta$ diketonato complexes - in situ generation of amphiphiles by water coordination". ${ }^{[182]}$ 


\subsubsection{Introduction}

A main focus of the work presented in this thesis is based on a non-conventional colloidal lithographic approach to create arrays of metal nanoparticles from metal-complex containing latex particles developed by Alfred Plettl and coworkers (chapter 3.4.3). ${ }^{[121]}$ A detailed description of the process is given in chapter 5.3. As a brief introduction, the process uses the metal-complex containing polymer latexes as sacrificial material. A plasma induced etching process is performed to completely combust the organic material of the colloids, leaving crude metal nanodots at the contact point of the colloids with the substrate. An annealing step is performed to transform the crude nanodots into crystalline metal nanoparticles. The size of the nanoparticles is controlled by the amount of metal complex in the colloids while the particle spacing is dictated by the initial diameter of the colloids. In order to demonstrate a precise control over size and lattice constant of the nanoparticles arrays, a precise adjustment of size and metal content of the polymer particles is a prerequisite. Both emulsion and miniemulsion polymerization can be applied in order to achieve the aforementioned goal. ${ }^{[26]}$

\subsubsection{Incorporation of metal complexes by emulsion polymerization}

In contrast to miniemulsion polymerization, emulsion polymerization is more limited both in the choice of monomers and functional molecules to be integrated into the latex particles. However, especially surfactant-free emulsion polymerization is priced for the high monodispersity ${ }^{[29,183]}$ of the resulting latex particles. These find applications where high precision colloidal crystals are needed, most prominently in the fields of photonics, ${ }^{[184-185]}$ phononics, ${ }^{[186-187]}$ and lithography. ${ }^{[5,136,188]}$

As the symmetry and order of the nanoparticles arrays to be produced from the metal-complex containing colloids crucially depends on the uniformity of the colloids, surfactant-free emulsion polymerization was applied to incorporate different metal complexes in order to produce colloids with a narrow size distribution.

Keeping in mind the diffusion based mechanism, strong deviations from the theoretical amount of metal in the colloids are expected. As already explained in detail in chapter 3.1.2 of the theory part, the latex particles are formed by diffusion of monomer to the polymerization loci formed by homogeneous nucleation in a surfactant free process. ${ }^{[30]}$ As the metal complexes, present in the monomer droplets at the beginning of the reaction, inherently feature a different diffusion coefficient in water compared to the monomer, the encapsulation efficiency can vary drastically. Figure 5.1.8.graphically illustrates the process. In the upper part, the metal complex 
(drawn as red star) possesses a relatively large diffusion coefficient as indicated by the size of the arrow. Therefore, a high amount of complex is found in the latex particles after polymerization. Figure 5.1.8b shows the same process for a complex with a smaller diffusion constant (green star; small arrow). Upon polymerization, the diffusion of complex to the polymerization loci is hindered and only a small amount of complex is found in the final latex particles. The stoichiometric encapsulation of two different complexes cannot be achieved by emulsion polymerization due to differences in diffusion coefficients. ${ }^{[26]}$ Different metal acetylacetonate complexes were tested with respect to the encapsulation efficiency. While platinum(II)acetylacetonate showed a partial incorporation that will be presented below, attempts to incorporate iron(III)acetylacetonate were completely unsuccessful as the emulsion was destabilized in the course of the polymerization. It is hypothesized that the tendency of the iron complex towards hydrolysis and condensation reactions at the interface interferes with the colloidal stability. A more detailed discussion will be presented in chapter 5.1.2.3.

a)
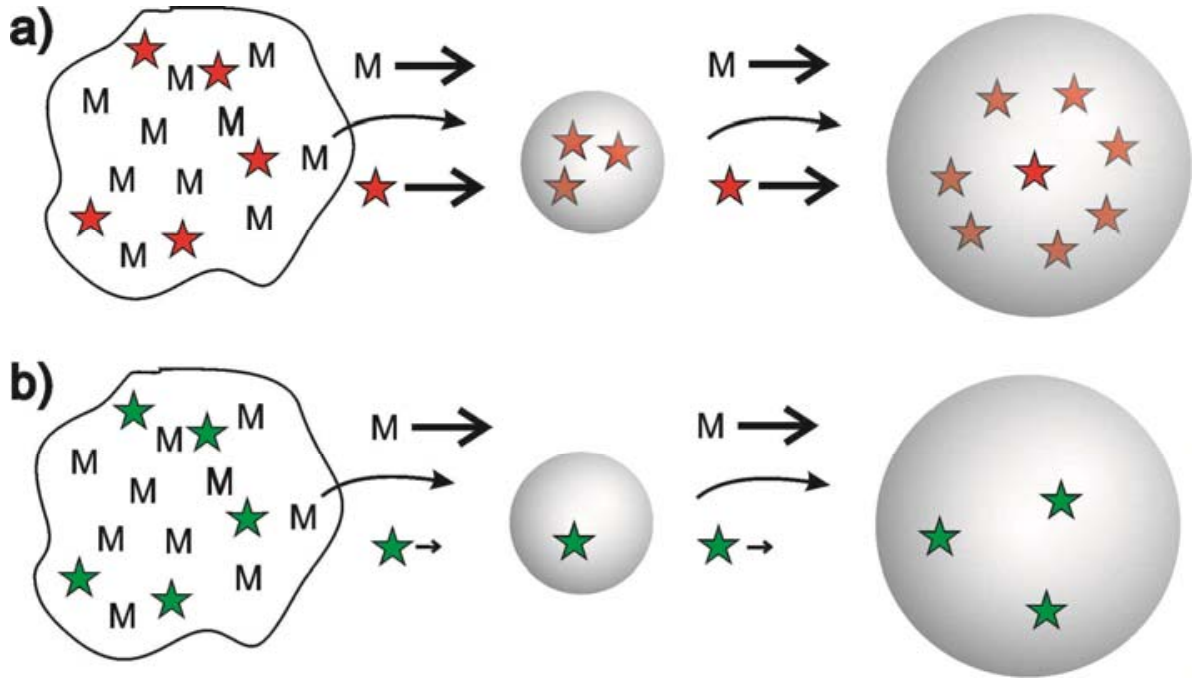

Figure 5.1.8. Emulsion polymerization in presence of metal-complex molecules with different diffusion constants indicated by the size of the arrows.

\subsection{Incorporation of platinum(II)acetylacetonate}

Figure 5.1.9 presents the results for the encapsulation of platinum(II)acetylacetonate in polystyrene latex particles by surfactant-free emulsion polymerization. Experimental details are given in the experimental section. A clear deviation between the amount of platinum added to the monomer phase and the amount measured in the latex particles after filtration and dialysis can be seen. The encapsulation efficiency ranges between 69 -and $78 \%$ of the theoretical value. 
However, as can be seen in the diagram on the right side of Figure 5.1.9b, the relationship between the amount of complex added to the monomer phase and the encapsulated amount of platinum is linear. Thus, an easy control over platinum content in the final particles is at hand. The size and homogeneity of the particles is not affected by the presence of the complex (compare Figure 5.1.9c).

a)

\begin{tabular}{ccccc}
\hline $\begin{array}{c}\text { Sample } \\
\#\end{array}$ & $\begin{array}{c}\text { Pt(acac) } \\
\text { added }\end{array}$ & $\begin{array}{c}\text { Pt-content } \\
\text { measured }\end{array}$ & $\begin{array}{c}\text { Diameter } \\
\text { Latex }\end{array}$ \\
\hline & $\begin{array}{c}\text { / wt.-\% of } \\
\text { monomer }\end{array}$ & $/$ wt. $\%$ & $\begin{array}{c}/ \% \text { of } \\
\text { theory }\end{array}$ & $/ \mathrm{nm}$ \\
\hline 1 & 1.0 & 0.39 & 69.2 & $175 \pm 7$ \\
2 & 2.0 & 0.75 & 75.5 & $196 \pm 5$ \\
3 & 4.0 & 1.37 & 78.5 & $185 \pm 5$ \\
\hline
\end{tabular}

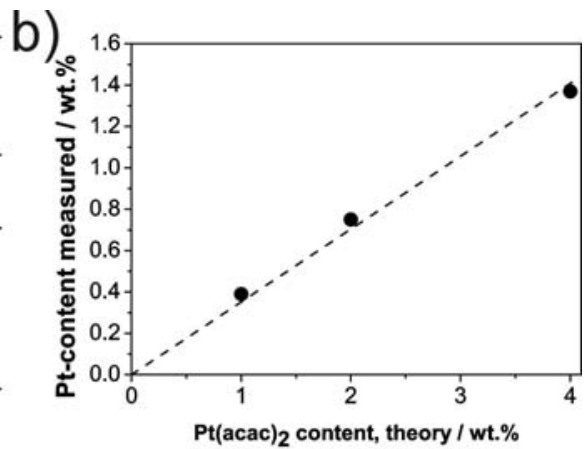

c)
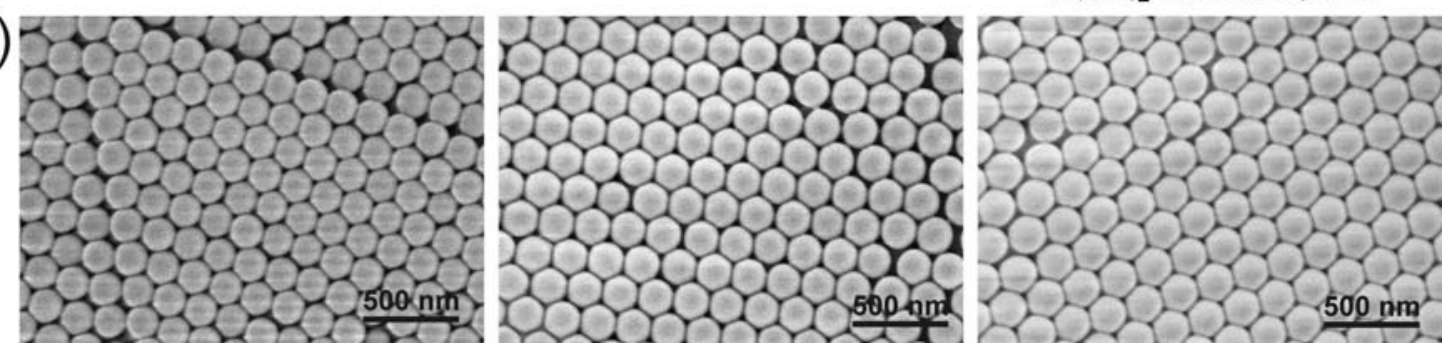

Figure 5.1.9. Encapsulation efficiency of platinum(II)acetylacetonate by surfactant-free emulsion polymerization of styrene. a) Table showing the details of the incorporation of platinum. b) Diagram showing the measured platinum content vs. the amount of platinum present in the monomer phase. c) Scanning electron micrographs of the respective particles: form left to right; $1 \% \mathrm{Pt}(\mathrm{acac})_{2}, 2 \% \mathrm{Pt}(\mathrm{acac})_{2}$, $4 \% \operatorname{Pt}(\mathrm{acac})_{2}$.

\subsubsection{Reasons for the different behavior of the metal complexes in incorporation reactions}

While the encapsulation of platinum(II)acetylacetonate as well as chromium(III)acetylacetonate and nickel(II)acetylacetonate (not shown) is possible with varying encapsulation efficiencies, the incorporation of iron(III)acetylacetonate by emulsion polymerization was completely impeded as the polymerization always yielded instable emulsions that phase separated in the course of the polymerization reaction. Previously, it has also been observed that the incorporation of different metal-acetylacetonate complexes by miniemulsion polymerization affected the size distribution of the resulting dispersions. ${ }^{[26]}$ Especially the presence of iron(III)acetylacetonate resulted in very broad size distributions compared to plain miniemulsions produced without added complexes. 
In order to resolve the differences, the metal complexes themselves were subjected to a more detailed study. First, their behavior at an oil-water interface was investigated to mimic the situation in an emulsion. Second, their stability towards hydrolysis in the presence of water was investigated. To resolve the influence of complexes on the interfacial properties in an emulsion, spinning drop tensiometry was used. In brief, a droplet of toluene containing defined amounts of metal complex was placed in a capillary filled with water and rotated at high rotational speeds. With the help of a camera and a software algorithm, the shape of the organic droplet can be used to calculate the interface tension of the two liquids (chapter 4.5). ${ }^{[172-173]}$ Toluene was chosen as organic phase as most of the $\beta$-diketonate complexes were readily soluble and because of the close chemical similarity to styrene. For a screening, acetylacetonate complexes of $3 \mathrm{~d}, 4 \mathrm{~d}$ and $5 \mathrm{~d}$ metals as well as complexes of the third main group were investigated. Figure 5.1.10 shows the measured interfacial tensions for toluene with metal-complex concentrations of $10 \mathrm{mmol} \cdot \mathrm{kg}^{-1}$ and $50 \mathrm{mmol} \cdot \mathrm{kg}^{-1}$ measured against water. Surprisingly, almost all transition metal complexes lowered the interface tension to a certain degree. This behavior was unexpected as the complexes are uncharged and highly symmetric. Usually, only asymmetric and amphiphilic molecules show a strong tendency to be present at an interface.
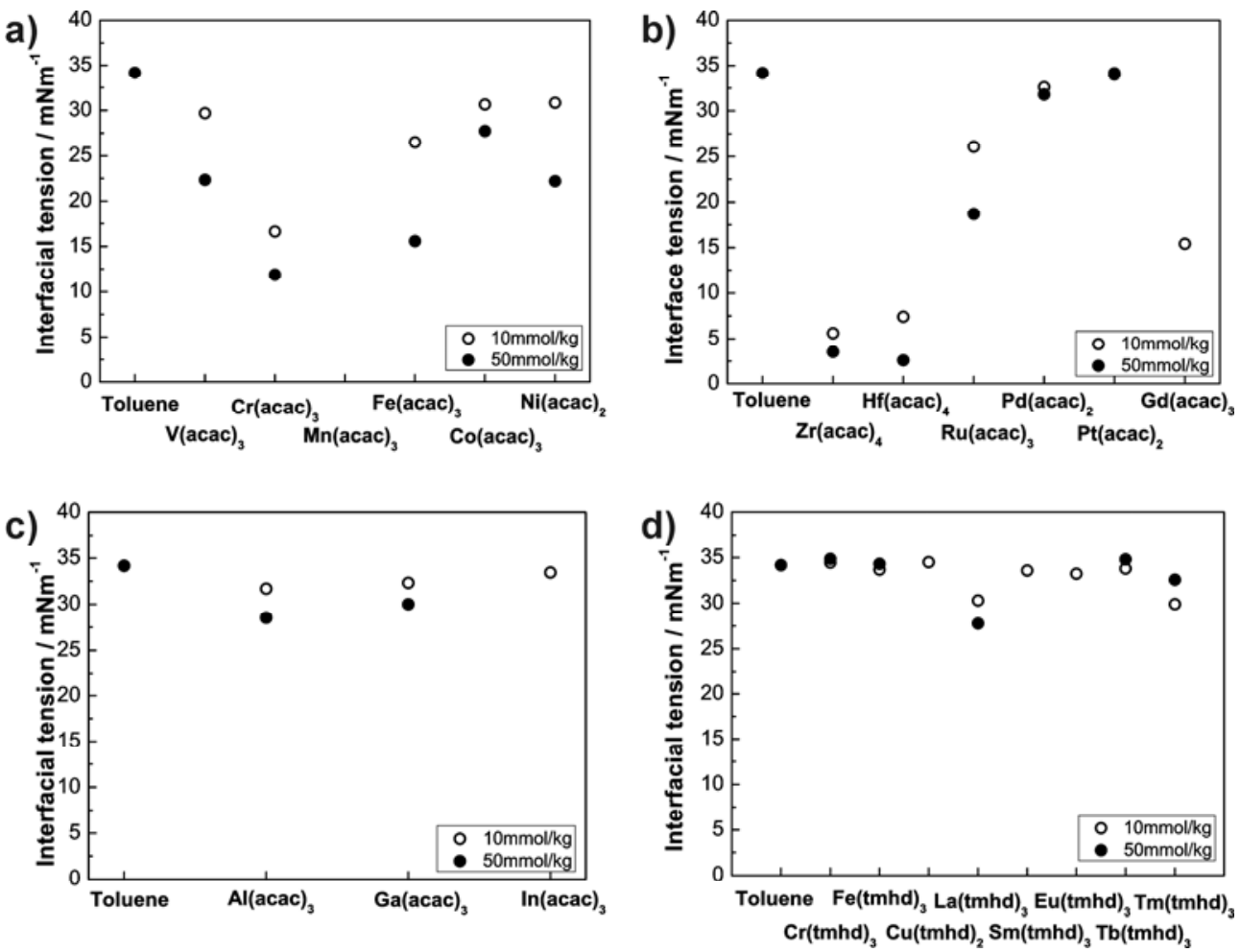

Figure 5.1.10. Interfacial tension of toluene solutions of the investigated metal- $\beta$-diketonate complexes against water as determined by spinning drop tensiometry. (a) acac complexes of $3 d$ metal cations, (b) acac complexes of $4 d / 5 d / f$ metal cations, (c) acac complexes of group 13 elements and (d) selected tmhdcomplexes. 
Most pronounced interfacial activities were measured for $\mathrm{Fe}(\mathrm{acac})_{3}$ (reduction from $34 \mathrm{mN} \cdot \mathrm{m}^{-1}$ to $15 \mathrm{mN} \cdot \mathrm{m}^{-1}$ for $\left.50 \mathrm{mmol} \cdot \mathrm{kg}^{-1}\right), \mathrm{Cr}(\mathrm{acac})_{3}\left(12 \mathrm{mN} \cdot \mathrm{m}^{-1}\right.$ for $\left.50 \mathrm{mmol} \cdot \mathrm{kg}^{-1}\right), \mathrm{Hf}(\mathrm{acac})_{4}$ and $\mathrm{Zr}(\mathrm{acac})_{4}$. The latter reduced the interfacial tension from $34 \mathrm{mN} \cdot \mathrm{m}^{-1}$ to only $4 \mathrm{mN} \cdot \mathrm{m}^{-1}$; a value that is nearly identical to results measured for sodium dodecyl sulfate (SDS) as an archetypical surface active molecule (maximum reduction to $4.5 \mathrm{mN} \cdot \mathrm{m}^{-1}$ for $8.6 \mathrm{mmol} \cdot \mathrm{kg}^{-1}$ ). 2,2-5,5 tetramethyl heptane dionato (tmhd) complexes on the contrary did not show interfacial activity (Figure 5.1.10d).

To explain the interfacial activity of the symmetric complex molecules, hydrogen bonding of water molecules to the complex was proposed. The addition of water is assumed to reduce the symmetry of the complexes and thus induce amphiphilicity and pin the molecules to the interface. In general, there are two ways in which a water molecule may coordinate to a complex that are illustrated in Figure 5.1.11: (1) inner sphere coordination, in which an incoming water molecule (partially) penetrates the ligand shell and coordinates to the central metal atom probably exchanging other ligands (Figure 5.1.11a), and (2) outer sphere coordination, in which incoming molecules primarily interact via the complex's ligands, ${ }^{[189]}$ for instance due to hydrogen bonding to the carbonyl oxygen (Figure 5.1.11b). Finally, a combination of both may also be possible (Figure 5.1.11c).

Scenario (1) is the basis for chemical reactions involving the metal center of a complex. Thus, inner-sphere coordination of water might trigger hydrolytic polycondensation processes, eventually leading to extended metal hydroxide/oxide structures. ${ }^{[190]}$ In scenario (2), the metal center may also play a crucial role. For instance, the coordination of $\beta$-diketonate ligands to $d$ metal cations makes them much stronger H-bond acceptors compared to free acetylacetone, since the cation polarizes the carbonyl bonds. ${ }^{[191]}$

(a)

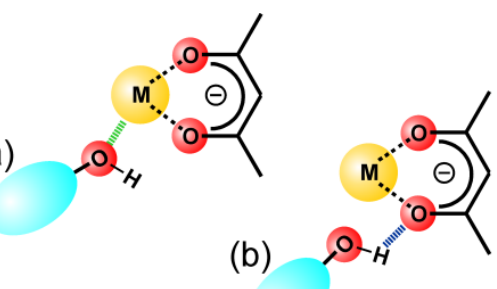

(c)

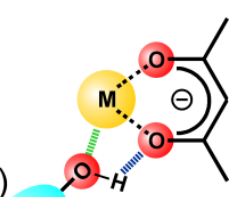

Figure 5.1.11. Different ways of coordination of an incoming (hydrogen-bonding) ligand to an $\mathrm{Me}(\mathrm{acac})_{x}$ complex: a) inner sphere, and b) outer sphere coordination, and c) a combination of both. 
To assess the role of hydrogen bonding in the generation of amphiphilic species, 3,5dichlorophenol (DCP) was added to the organic phase containing the metal complexes. Phenols are much stronger H-donors compared to water. ${ }^{[192]}$ Thus, it was hypothesized that if hydrogen bonding plays an important role for the interfacial activity, a strong hydrogen donor in the organic phase may compete with water in binding to the complexes. As the phenol is the stronger donor, the formation of phenol-complex adducts would be energetically favorable. The phenol itself is soluble in the organic phase only. Therefore, the bonding to the complex would not generate an amphiphilic species and consequently lower the interfacial tension. The measured interfacial tension should be higher compared to a system without 3,5-dichlorophenol. Figure 5.1.12 presents measurements of the interfacial tensions for the most active complexes $\mathrm{Cr}(\mathrm{acac})_{3}, \mathrm{Fe}(\mathrm{acac})_{3}$ and $\mathrm{Zr}(\mathrm{acac})_{4}$ in presence of different concentrations of dichlorophenol (DCP). A clear increase in interfacial tension in presence of DCP is detected, thus corroborating with the proposed model. This model also explains why tmhd complexes do not show interfacial activity. The presence of the bulky methyl groups effectively shields the carbonyl atoms from the hydrogen donors.
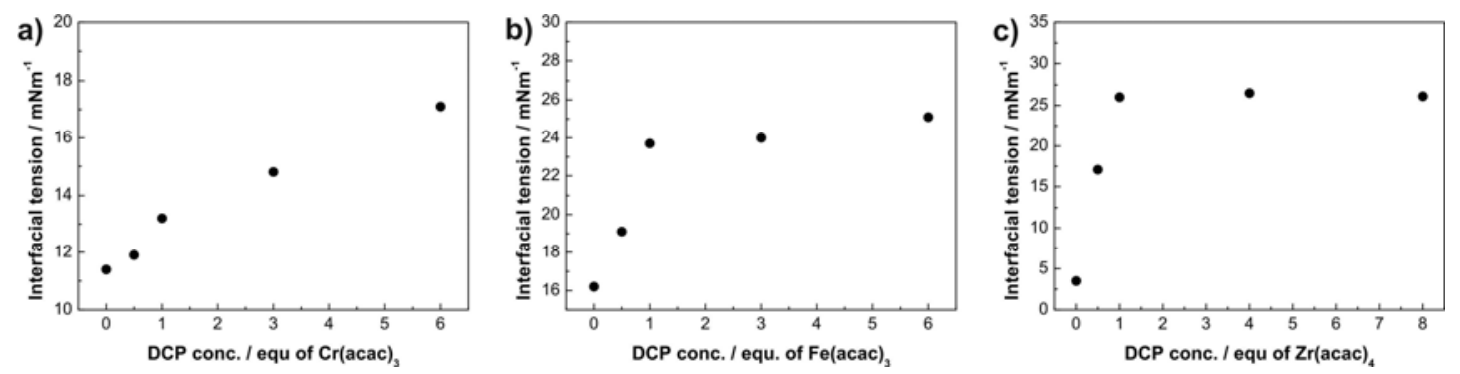

Figure 5.1.12. Effect of the presence of DCP on the interfacial tension of toluene solutions of (a) $\mathrm{Cr}(\mathrm{acac})_{3}$, (b) $\mathrm{Fe}(\mathrm{acac})_{3}$ and (c) $\mathrm{Zr}(\mathrm{acac})_{4}$ at $50 \mathrm{mmol} \cdot \mathrm{kg}^{-1}$ against water as a function of the number of molar equivalents of 3,4-dichlorophenol (DCP) added.

A further assessment of the mechanism was performed by Dr. Coenrad von den Brom who used diffusion ordered NMR spectroscopy (DOSY-NMR) to investigate which coordinating mechanism (1 or 2 of Figure 5.1.11) takes place with the metal complexes.

Diffusion ordered NMR spectroscopy revealed that $\mathrm{Zr}(\mathrm{acac})_{4}$ adds water directly at the metal center under loss of one of the ligands. Subsequently, hydrolysis takes place and larger aggregates are formed. The investigation of $\mathrm{Fe}(\mathrm{acac})_{3}$ as well as $\mathrm{Cr}(\mathrm{acac})_{3}$ was not possible with NMR based methods due to the paramagnetism of both complexes. The complete set of data including the DOSY experiments can be found in the article "interfacial activity of uncharged, highly symmetric metal $\beta$-diketonato complexes - in situ generation of amphiphiles by water coordination" that has recently been accepted for publication in Langmuir. 
An important indication towards the occurrence of hydrolytic reactions upon contacting $\mathrm{Fe}(\mathrm{acac})_{3}$ in toluene with water was provided by visual inspection of the samples after spinning drop tensiometry. In the presence of $\mathrm{Fe}(\mathrm{acac})_{3} \mathrm{Zr}(\mathrm{acac})_{4}$ a thin skin was formed around the toluene droplet whilst the capillary was spun, which effectively stopped the droplet from regaining a spherical shape after spinning (Figure 5.1.13). The same effect was observed for $\operatorname{Zr}(\mathrm{acac})_{4}$ containing toluene droplets surrounded by water. In contrast, no skin formation was observed in the presence of $\mathrm{Cr}(\mathrm{acac})_{3}$. Indeed, aqueous transition metal ions, especially small and highly charged cations are well-known for their hydrolytic polycondensation. ${ }^{[193]}$ During polycondensation, extended $[-\mathrm{M}-(\mathrm{OH})-]_{n}$ or $[\mathrm{M}-\mathrm{O}-]_{\mathrm{n}}$ chains or networks are formed, which may react further and mineralize to a form of metal oxide. We tentatively suggest that some form of polycondensation led to the formation of the observed skin. Interestingly, this skin formation was not observed in the presence of dichlorophenole (Figure 5.1.13b and d), consistent with the hypothesis that the phenole reduces the water-complex interaction by forming adducts with the complex itself. These observations correlate well with the hydrolysis constants of the respective aqua ions. $\mathrm{Zr}^{4+}$ with a $\mathrm{pK}_{\text {hydr }}$ of 0.22 has the highest tendency to hydrolyze, followed by $\mathrm{Fe}^{3+}$ with $\mathrm{pK}_{\text {hydr }}=2.19$. In contrast, $\mathrm{Cr}^{3+}$ with $\mathrm{pK}_{\mathrm{hydr}}=4.01$ has a hydrolysis constant 100 times lower than $\mathrm{Fe}^{3+}$ Thus, the tendency of $\mathrm{Cr}^{3+}$ for hydrolysis is the lowest of these ions. ${ }^{[190]}$
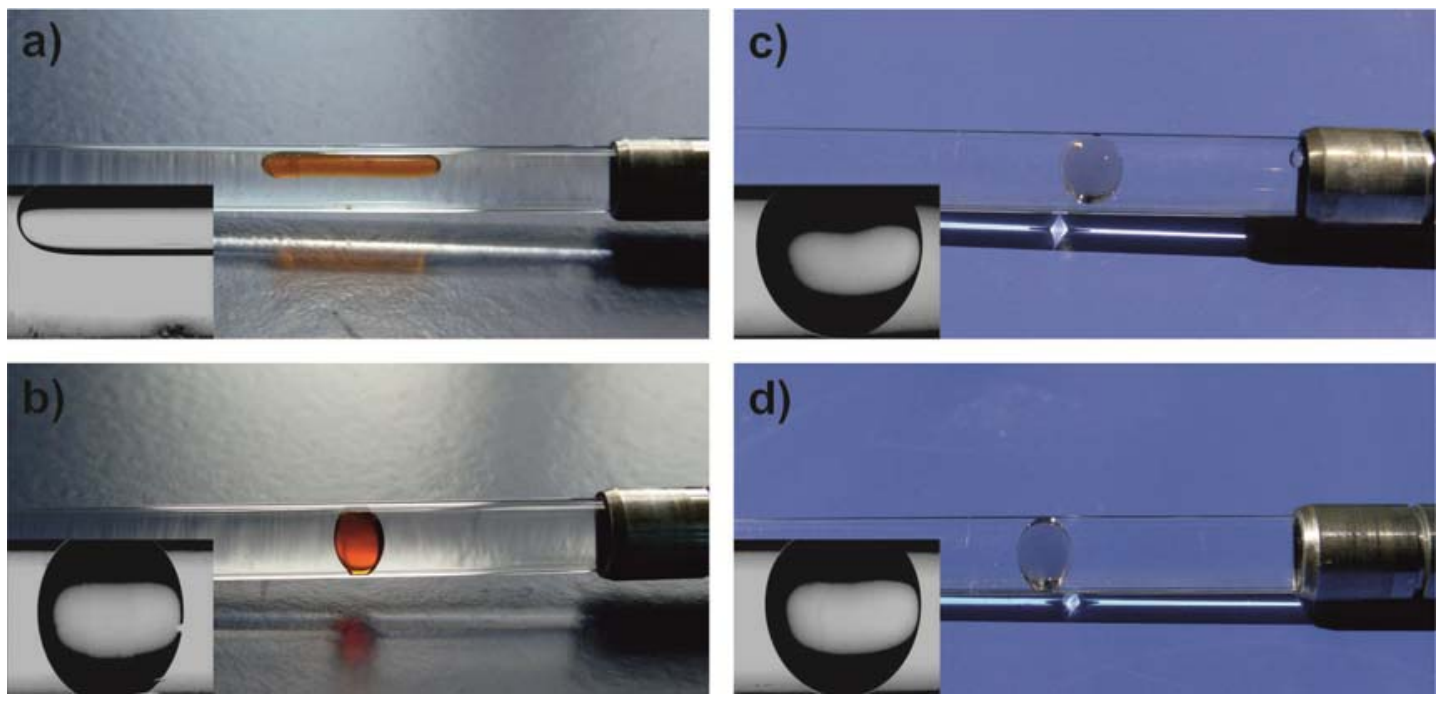

Figure 5.1.13. Toluene solutions of $\mathrm{Me}(\mathrm{acac})_{3}$ complexes after spinning drop tensiometry measurements. (a) Shape retention indicatives skin formation for $\mathrm{Fe}(\mathrm{acac})_{3}\left(50 \mathrm{mmol} \cdot \mathrm{kg}^{-1}\right)$, (b) no shape retention (i.e. restoration of spherical droplet after spinning) for $\mathrm{Fe}(\mathrm{acac})_{3}$ in the presence of dichlorophenole in the toluene phase; (c) Shape restoration for $\mathrm{Cr}(\mathrm{acac})_{3}$; and (d) shape restoration for $\mathrm{Cr}(\mathrm{acac})_{3}+\mathrm{DCP}$. 
Concluding, the differences in emulsion polymerization in presence of different metal complexes can be traced back to the differences in behavior and reactivity of the complexes at the interface. Especially hydrolysis reactions apparently induce instabilities of the emulsion during emulsion polymerization in presence of $\mathrm{Fe}(\mathrm{acac})_{3}$.

\subsubsection{Incorporation of metal complexes by miniemulsion polymerization}

Miniemulsion polymerization is the method of choice for a controlled encapsulation of functional molecules into polymeric nanoparticles. The mechanism and various examples of functional molecules that were successfully incorporated are described in detail in chapter 3.1.1 of the theory part. In this thesis, the miniemulsion process was primarily applied for the simultaneous encapsulation of iron(III)acetylacetonate and platinum(II)acetylacetonate. An investigation on the incorporation of a variety of different metal complexes and the resulting morphology of the particles has been conducted by Schreiber et al. ${ }^{[26]}$

For clarity, the process scheme of a miniemulsion polymerization for the loading of polymeric nanoparticles with iron and platinum complexes is shown in Figure 5.1.14.

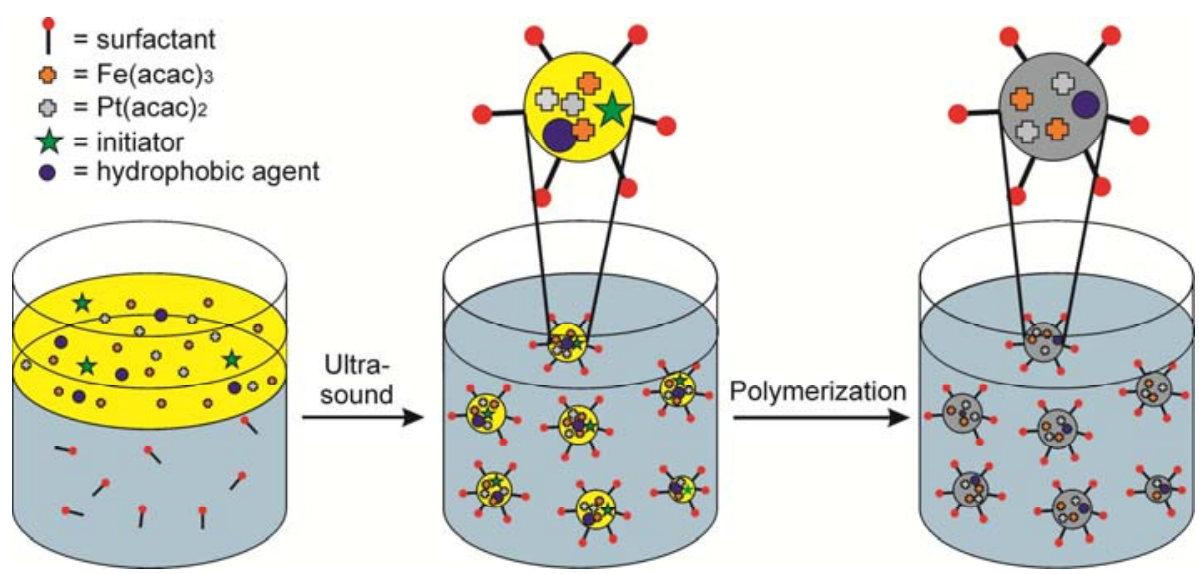

Figure 5.1.14. Schematic representation of the miniemulsion process used for the fabrication of metalcomplex loaded latex particles.

For the encapsulation of two different metal complexes in a fixed/desired ratio, it is of crucial importance to have individual nanoreactors during the polymerization in order to retain the correct stoichiometry in the latex particles. This is a key difference to emulsion polymerization where diffusion of monomer to the polymerization loci takes place. ${ }^{[28,30]}$ As functional 
molecules added to the monomer will feature different diffusion coefficients, a stoichiometric encapsulation is impeded.

\subsection{Incorporation of stoichiometric amounts of $\mathrm{Fe}(\text { acac })_{3}$ and $\mathrm{Pt}(\mathrm{acac})_{2}$}

A variety of particles with different sizes and metal contents were synthesized and characterized with respect to their metal content using the newly developed inductively coupled plasmaoptical emission spectrometry (ICP-OES) method (chapter 5.1.1). ${ }^{[174]}$ The characterization and reaction parameters are shown in the experimental section. The encapsulation efficiency of the two metal complexes was of primary interest for the subsequent application of the latex particles in the etching process to create ferromagnetic FePt alloy nanoparticles. For the successful subsequent conversion into inorganic FePt nanoparticles, it is of crucial importance to tightly control the encapsulation of both iron and platinum precursor complexes in a way that the stoichiometry in the miniemulsion particles is close to the desired 1:1 ratio. Figure 5.1.15 presents the measured metal contents for polystyrene particles exemplarily for two different SDS concentrations of $1 \mathrm{wt} .-\%$ (left row) and $5 \mathrm{wt} .-\%$ (right row) with respect to the monomer. Further miniemulsions with varying SDS contents dispersions have been synthesized as well and showed similar efficiencies as the ones presented exemplarily (the complete set of data is shown in the experimental section). The diagrams show the measured metal content against the metal complex concentration in the initial monomer phase. The metal content of the pristine dispersions after polymerization are shown in Figure 5.1.15a and d.

From miniemulsion theory, the encapsulation efficiency of both complexes should generally be close to the theoretical value of $100 \%$ that is marked by a dotted line in the diagrams. For the samples prepared with $1 \mathrm{wt} . \mathrm{\%}$ SDS, this is valid for complex concentrations of 13 and $26 \mathrm{mmol} / \mathrm{kg}$ (equaling approx. 0.5 and $1 \mathrm{wt} .-\%$ of platinum complex). At higher complex concentrations, platinum complex is lost during the polymerization. The iron complex remains completely encapsulated. When preparing miniemulsions with $5 \mathrm{wt} .-\%$ SDS (right row), a complete encapsulation of both complexes is detected for both iron and platinumacetylacetonate for all concentrations used. Obviously, higher SDS amounts lead to a better incorporation behavior of the complexes. The reasons for this behavior remain unclear and merit further investigations. 

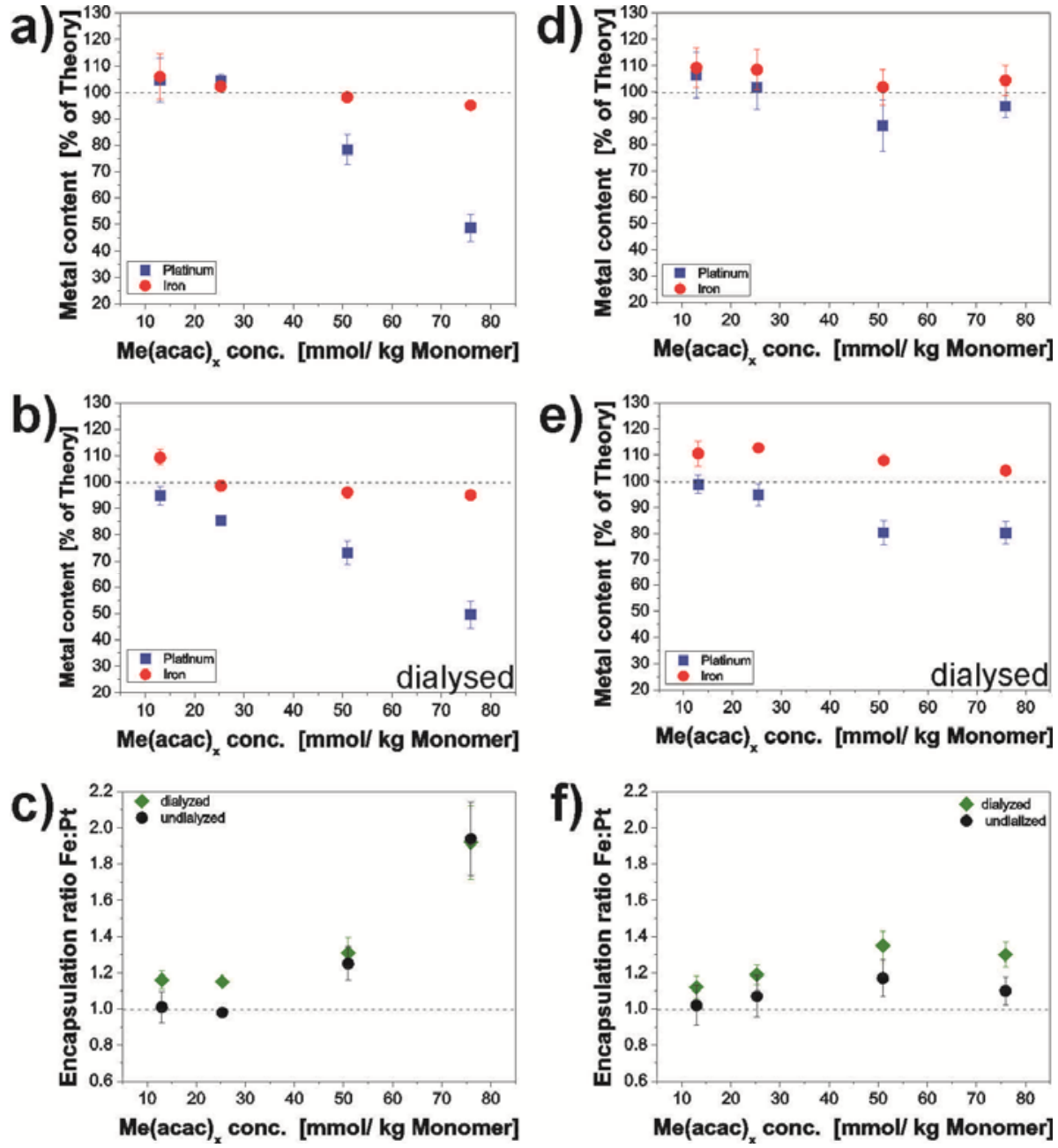

Figure 5.1.15. Encapsulation efficiencies of $\mathrm{Fe}(\mathrm{acac})_{3}$ and $\mathrm{Pt}(\mathrm{acac})_{2}$ in the miniemulsion process with 1 wt.- $\%$ SDS with respect to the monomer (a-c) and 5 wt.- $\%$ SDS (d-f). a,d) show the results for the dispersion after polymerization; $\mathrm{b}, \mathrm{e}$ ) the metal contents after dialysis and $\mathrm{c}, \mathrm{f}$ ) the ratios of Fe:Pt as calculated from the measurements.

It has to be noted that values slightly above $100 \%$ efficiency can be detected if the polymerization is not fully completed as unreacted monomer will evaporate during solid content determination needed for the calculation of the relative encapsulation efficiency. Figure 5.1.15b and e give the results for the particle dispersions after extensive dialysis (dialyzed until the conductivity of the water phase after several hours of dialysis remained close to the value for the pure water used; typically after dialysis with approximately 81 of water). Surprisingly, for all dispersions investigated, a loss of about $15 \%$ of the platinum complex is observed. This observation that is in contradiction to the theory of miniemulsion will be investigated in detail below.

Finally, Figure 5.1.15c and $\mathrm{f}$ present the encapsulation Fe:Pt ratio in the particles as calculated from the ICP-OES measurements. Within the experimental error, the pristine dispersions feature a stochiometric 1:1 content of iron and platinum, with the exception of high complex 
concentrations ( 2 and 3 wt.- $\%$ of $\operatorname{Pt}(\mathrm{acac})_{2}$ ) with 1 wt.- $\%$ SDS. Extensive cleaning, as desired for the subsequent application to remove e.g. excess SDS, leads to non-stochiometric ratios. This is undesired for the conversion into FePt alloy nanoparticles as the ferromagnetic $\mathrm{L} 1_{0}$ phase only forms with nearly perfectly matching ratios.

\subsection{Stoichiometric FePt-precursor particles by unstoichiometric encapsulation}

In order to achieve the required stoichiometry of Fe and Pt of 1:1 and still produce clean, dialysed particles without SDS impurities, a simple strategy was pursued:

Knowing that the loss of platinum complex is systematically close to $15 \%$ in all samples, dispersions with a non-stoichiometric Fe-Pt ratio can be synthesized with a small excess of platinum-acetylacetonate (15\%), thus compensating the loss during dialysis. Figure 5.1.16 gives an example of the concept.

To compensate the $\mathrm{Pt}$ loss after dialysis, the initial $\mathrm{Fe}: \mathrm{Pt}$ ratio was chosen as 0.85 $\left(21.5 \mathrm{mmol} / \mathrm{kg} \mathrm{Fe}(\mathrm{acac})_{3}\right.$ and $\left.25.3 \mathrm{mmol} / \mathrm{kg} \mathrm{Pt}(\mathrm{acac})_{2}\right)$. Figure 5.1.16a shows the results for the ICP-OES metal content determination. The abscissa reflects the difference in nominal metal content between iron and platinum. The left ordinate presents the results for the measurement. It shows that the value determined does not differ from the theoretical expectation within the experimental accuracy, in agreement with the results shown before. The right ordinate shows the Fe:Pt ratio as calculated from the metal contents measured. It gives a value of 0.85 , reflecting the ratio chosen initially.

Figure 5.1.16b depicts the metal contents for the same sample after extensive dialysis (dialysis until the conductivity was equal to the one of pure water). Corroborating the findings before (Figure 5.1.15), no change in iron concentration is detected and the determined value still is around $105 \%$ of theory. As expected, the platinum content dropped after dialysis to $86 \%$ of the theoretical value. The Fe:Pt ratio calculated, shown on the right ordinate, now yields a value of $1.04 \pm 0.04$ and is thus very close to the desired $1: 1$ stochiometry for the creation of FePt nanoparticles. 

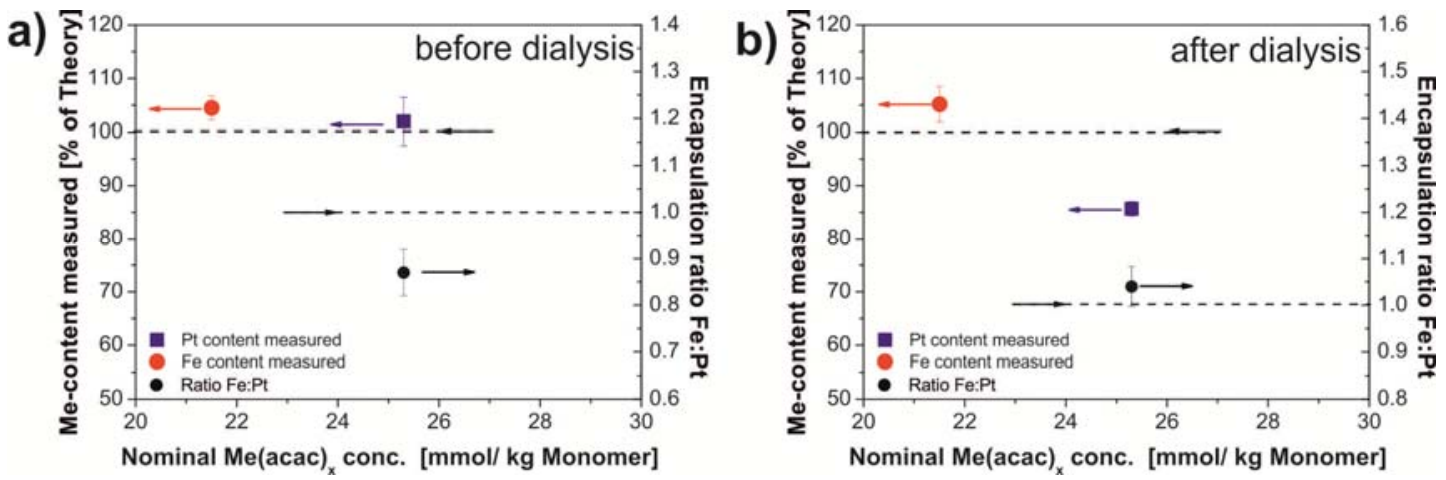

Figure 5.1.16. Production of latex particles with stochiometric Fe:Pt ratios after extensive cleaning by dialysis. The loss of platinum during the dialysis is compensated by an excess of $\mathrm{Pt}(\mathrm{acac})_{2}$ in the initial monomer phase. The dotted lines represent the theoretical value of $100 \%$ encapsulation and the perfect ratio Fe:Pt of 1.0. a) Dispersion as measured directly after polymerization. b) Dispersion measured after dialysis.

\subsection{Investigations on the loss of platinum in the miniemulsion process}

A detailed ICP-OES analysis of the metal concentration at different stages of the miniemulsion polymerization process was performed with the aim to resolve at which stage of the reaction the loss of platinum occurs. The investigation was executed as follows. Standard miniemulsions containing one percent $\mathrm{Pt}(\mathrm{acac})_{2}$ alone and a stoichiometric mixture of one percent $\mathrm{Pt}(\mathrm{acac})_{2}$ and an equimolar amount (0.89 wt.-\%; equaling $25.3 \mathrm{mmol} \cdot \mathrm{kg}^{-1}$ monomer) of $\mathrm{Fe}(\mathrm{acac})_{3}$ were prepared. At each step of the reaction, namely before and after polymerization, a part of the emulsified heterophase system was collapsed with concentrated calcium chloride solution $(c=1$ $\mathrm{mol} \cdot \mathrm{l}^{-1}$ ). The addition caused a sudden collapse of the particle droplets induced by strongly screened electrostatic repulsion. In the emulsion state before polymerization, the system thus phase separated and the water phase was isolated. The dispersion after polymerization was filtered to obtain the pure water phase. The metal concentrations in the collected water phases were investigated by ICP-OES. Finally, the dispersions were extensively dialyzed using one liter of ultrapure water that was exchanged every $48 \mathrm{~h}$. In total, the water was changed 13 times, adding to a total of approximately one month of dialysis time. For comparison, the typical dialysis protocol performed for the experiments above usually took a week and typically involved less than eight changes of water.

Table 5.1.6 presents the results of the ICP-OES investigation. All metal concentrations are given as percent of theory to facilitate the discussion. The water phases before and after polymerization did not contain significant amounts of metal complexes: both metal complexes in the two reactions under investigation are only found in concentrations below one percent in the water phases. In a reverse conclusion, this means that (almost) the complete amount of metal 
must be present in the organic phase prior polymerization and in or at the surface of the particles after polymerization. Therefore, it can be concluded that the established miniemulsion mechanism as presented in the theory part seems to be valid for the incorporation of metal complexes.

Table 5.1.6. ICP-OES investigation on the presence of metal complexes at different stages of the miniemulsion polymerization reaction.

\begin{tabular}{cccccc}
\hline Sample & $\begin{array}{c}\mathbf{M e}(\mathbf{a c a c})_{\mathbf{x}} \\
\text { Theory } / \text { wt.- } \%\end{array}$ & $\begin{array}{c}\mathbf{M e}(\mathrm{acac})_{\mathbf{x}} \\
\text { Theory } / \mathbf{m m o l} / \mathbf{k g} \text { styrene }\end{array}$ & $\begin{array}{c}\text { Emulsion } \\
\text { water-Phase }\end{array}$ & $\begin{array}{c}\text { Dispersion } \\
\text { water-Phase }\end{array}$ & $\begin{array}{c}\text { Dispersion } \\
\text { total }\end{array}$ \\
\hline $\mathbf{P t}$ & $1.00\left(\mathrm{Pt}(\mathrm{acac})_{2}\right)$ & $25.3\left(\mathrm{Pt}(\mathrm{acac})_{2}\right)$ & $0.42 \%$ & $0.87 \%$ & $98.4 \%$ \\
FePt & $1.00\left(\mathrm{Pt}(\mathrm{acac})_{2}\right)$ & $25.3\left(\mathrm{Pt}(\mathrm{acac})_{2}\right)$ & $0.36 \%$ & $0.89 \%$ & $99.1 \%$ \\
FePt & $0.89\left(\mathrm{Fe}(\mathrm{acac})_{3}\right)$ & $25.3\left(\mathrm{Fe}(\mathrm{acac})_{3}\right)$ & $0.87 \%$ & $0.17 \%$ & $105.5 \%$ \\
\hline
\end{tabular}
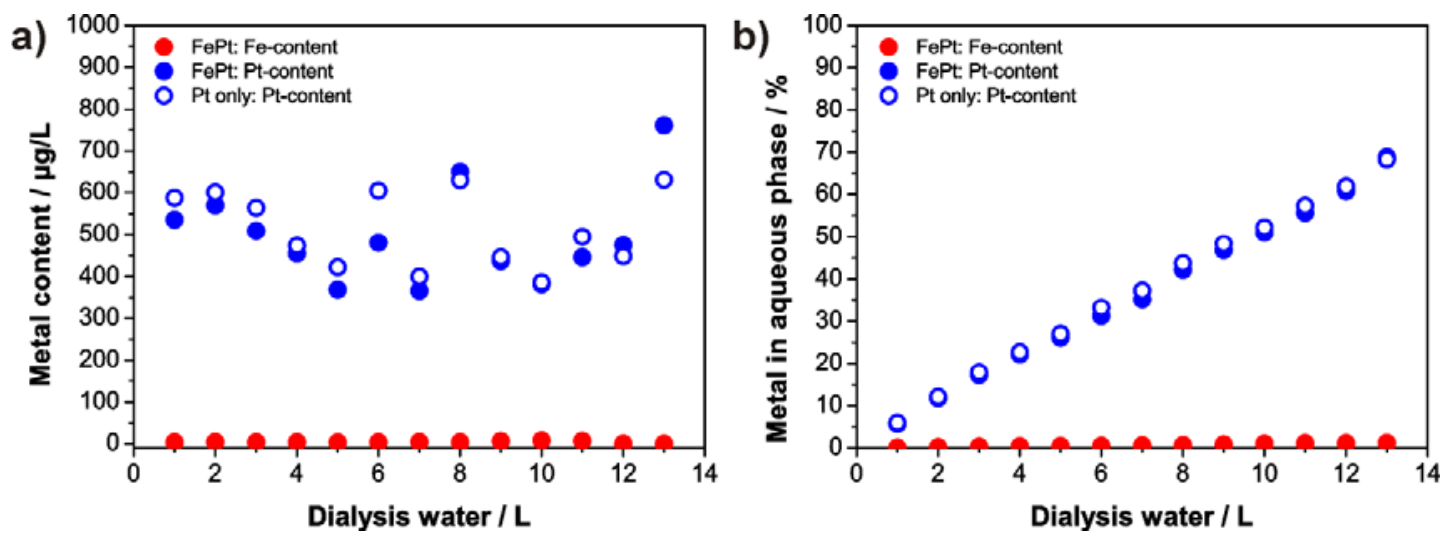

Figure 5.1.17. ICP-OES analysis of the loss of metal complexes during dialysis of dispersions containing $\mathrm{Pt}(\mathrm{acac})_{2}$ only as well as $\mathrm{Pt}(\mathrm{acac})_{2}$ and $\mathrm{Fe}(\mathrm{acac})_{3}$ simultaneously. a) Metal content detected in the aqueous phase. b) Metal content, plotted in percent of the total amount of metal initially present in the dispersion.

Figure 5.1.17 shows the results of the elemental analysis of the water phase in the course of the dialysis. Dramatic differences between iron(III)acetylacetonate and platinum(II)acetylacetonate can be seen. While no iron is detected throughout the complete dialysis protocol, there is a constant concentration of platinum detected in each sample of the dialysis water measured. The loss of platinum that diffuses into the water phase sums up linearly to approximately $70 \%$ of the theoretical value for the duration of the dialysis experiment. Judging from the strict linear dependency, it can be speculated that the remaining amount of platinum in the particles could be removed as well; had the dialysis been performed for an increased period of time. The behavior is very unexpected as both complexes feature a very limited water solubility (the better soluble $\mathrm{Fe}(\mathrm{acac})_{3}$ has a solubility of $215 \mathrm{ppm}^{[194]}$ ). 
It is speculated that the absence of iron might be related to the formation of larger structures as has been evidenced by hydrolysis reactions forming a shell around the $\mathrm{Fe}(\mathrm{acac})_{3}$ containing toluene phase in spinning drop experiments (chapter 5.1.2.3). This might prevent diffusion of the larger aggregates into the water phase while the more inert platinum complex might be subjected to diffusion into the water phase. Even though the solubility is limited, the large amounts of water applied might account for the loss. Alternatively, (partial) complex degradation during the sonication reaction cannot be excluded at present and might produce more readily water soluble species. The value of approximately $15 \%$ loss of platinum reported before does not seem to be a specific property of the platinum incorporation process but rather a coincidence arising from the dialysis protocol that was used throughout the experiments mentioned above.

The exact mechanism of the loss of platinum during dialysis certainly merits further investigations and additional experimental data for the behavior of different complexes might help to resolve the question. For the miniemulsion polymerization itself, evidence from the ICP measurements suggests a complete incorporation of both complexes in agreement with the established mechanism.

\subsubsection{Seeded emulsion polymerization as a tool to control the size of polymeric nanoparticles}

Seeded emulsion polymerization can be used to combine the benefits of miniemulsion and emulsion polymerization. In the process, metal-complex containing polymer particles, as synthesized by either of the aforementioned methods, are used as seed particles. Subsequent addition of monomer in the presence of initiator leads to an increase in size of the seed particles in an emulsion-like mechanism described in detail below. By varying the amount of monomer added and carefully controlling the reaction conditions, the size of the final particles can be precisely adjusted. As a desired side-effect in the reaction, the relative polydispersity of the colloids decreases as a result of the increase in colloid diameter.

In the first part of the chapter, platinum containing particles synthesized by miniemulsion polymerization are used in the seeding process to investigate the influence of principal reaction parameters on the resulting particles dispersions. Finally, $\mathrm{Pt}(\mathrm{acac})_{2}$ containing particles were synthesized by emulsion polymerization and carefully increased in size to cover ranges from $130 \mathrm{~nm}$ to $250 \mathrm{~nm}$ in order to be applied in the etching process in chapter 5.3. 


\subsection{Detailed examination of the influence of reaction parameters on the resulting particle dispersions}

For the detailed investigation of the influences of the different reaction parameters, it is essential to first reflect on the reaction mechanism in detail. The focus of the study presented lies in tuning the reaction in a way as to avoid secondary nucleation. Secondary nucleated particles are colloids formed newly in the course of the reaction similarly as latex particles are generated in a conventional emulsion polymerization. ${ }^{[30-31]}$ Given that the seeded emulsion polymerization is applied to adjust the size of functional colloidal particles, secondary nucleation must be prevented in order to guarantee that all particles bear the desired functionality.

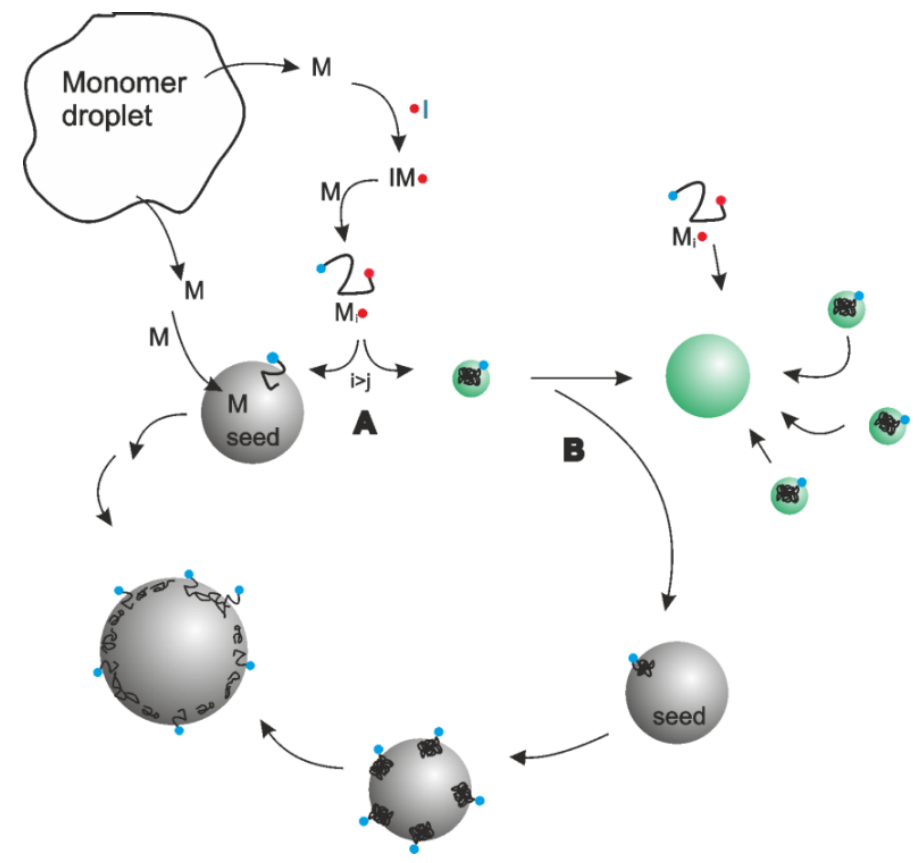

Figure 5.1.18. Simplified model of a seeded emulsion polymerization process to clarify the desired reaction pathway.

Figure 5.1.18 gives a simplified picture of the processes involved. After being injected into the water, the monomer diffuses through the water phase to the hydrophobic seed particles present that thus, represent a monomer rich area. The water-soluble initiator thermally decomposes to form radicals in the water phase. These start to polymerize monomer molecules present in the aqueous phase to form oligoradicals $\mathrm{IM}_{\mathrm{i}}$. Due to the ionic headgroup introduced by the persulfate radical of the initiator (ammonium peroxodisulfate, APS), the oligoradicals remain water soluble until they reach a critical chain length $\mathrm{IM}_{\mathrm{j}} \cdot$ upon which they become insoluble in water. ${ }^{[31,195]}$ For styrene, this length was determined to be 5 monomer units. ${ }^{[195-196]}$ The fate of the aqueous oligoradical and therefore of the course of reaction is determined by whether or not 
it enters a seed particle before reaching the critical chain length. This point is marked as A in the scheme. Entering a seed particle gives the radical access to the monomer reservoir present in the particle where it subsequently polymerizes, resulting in a size-increased seed particle (Figure 5.1.18, left side). Assuming the oligoradical does not meet a seed particle before adding the last monomer unit necessary to exceed the critical chain length, the chain becomes insoluble in the water phase and forms a particle nucleus by a coil to globule transition (this pathway of the reaction is shown on the right side of the scheme). ${ }^{[30,195,197]}$ This particle nucleus itself is not stable as it features only one charge coming from the initiator. Stabilization can be achieved by two different pathways, marked with a B in the scheme. First, the particle nucleus can attach to a seed particle where it eventually will be completely incorporated in the course of reaction due to more monomer diffusion to the particle. In this case, no secondary particle will appear in the final dispersion. ${ }^{[30-31]}$ On the other hand, several particle nuclei can cluster together to form a stable particle when the charge density on their surface becomes sufficiently high. ${ }^{[30,195,197]}$ This particle, termed secondary particle (shown in green color), now participates in the reaction as a new seed particle. In order to avoid secondary nucleation forming unwanted, plain particles, the latter pathway needs to be avoided. Several factors influence the course of the reaction; predominantly the seed concentration and the concentration of surfactant added to the reaction. Furthermore, the initiator concentration and the composition of the continuous phase are of importance for the fate of the radical and particle nuclei as well. Various studies have been published, especially on the role of seed particle concentration. ${ }^{[198-199]}$ It has been found that concentrations above $10^{14}$ particles per liter are sufficient to avoid the formation of particle nuclei. ${ }^{[198]}$ Therefore, for all reactions performed, the seed particle concentration was fixed to $2.65 \cdot 10^{14}$ particles per liter and the effect of other reaction parameters were investigated.

Furthermore, in all cases styrene was added dropwise, very slowly with a speed of $1 \mathrm{ml} / \mathrm{h}$. That ensures that monomer is directly consumed once it appears in the reaction mixtures (monomer starved condition ${ }^{[200]}$ and no monomer reservoir is present that might chance the reaction pathway towards secondary nucleation in a classical emulsion polymerization way.

The functional seed particles for the investigation presented were synthesized by a standard miniemulsion process described in the experimental section. The process resulted in polystyrene latex particles with a size of $167 \pm 14 \mathrm{~nm}$ and a platinum acetylacetonate load of $1 \mathrm{wt} . \mathrm{\%}$. A representative scanning electron microscopy image of the particles is shown in Figure 5.1.19. All seeded emulsion polymerization reactions were executed as follows if not stated otherwise. A dispersion of $0.1 \mathrm{wt} .-\%$ of seed particles with $0.01 \mathrm{wt} .-\%$ of SDS (relative to the amount of water used) was heated to $75^{\circ} \mathrm{C}$. Ammonium peroxodisulfate, (APS, $\left(\mathrm{NH}_{4}\right)_{2} \mathrm{~S}_{2} \mathrm{O}_{8}$ ), used as initiator, was dissolved in a small amount of ultra-pure water and added to the dispersion. Styrene as monomer was added to the solution using a syringe pump with a flow rate of 
$1 \mathrm{ml} \cdot \mathrm{h}^{-1}$. The reaction was stirred for $24 \mathrm{~h}$ at $80^{\circ} \mathrm{C}$ under argon atmosphere. After completion, the resulting particles were dialyzed in order to remove unreacted initiator and monomer. In the previous chapter (5.1.1.2.3), it has been shown by inductively coupled plasma optical emission spectrometry (ICP-OES) measurements that a seeded emulsion polymerization process does not change the amount of platinum acetylacetonate in the particle. ${ }^{[174]}$

\subsection{Variation of the amount of monomer added}

In a first set of experiments, the amount of monomer added was varied while all other parameters remained constant. These experiments aimed at investigating the size range available for the seeded emulsion polymerization. The amount of monomer added is described as the normalized quantity monomer excess and describes the mass of styrene added relative to the total mass of seed particles $\left(m_{\text {styrene }} / m_{\text {seed }}\right)$. Please note that the total amount of initiator was unchanged, leading to a constant initiator concentration in the water phase but drastically changing the ratio of initiator to monomer added. Table 5.1.7 presents the reaction details, Figure 5.1.19 shows the dependency of the amount of monomer added on the resulting particle size.

Table 5.1.7 Reaction details for the set of experiments shown in Figure 5.1.19. All reactions were carried out with 0.1 wt.- $\%$ dispersions of seed particles.

\begin{tabular}{|c|c|c|c|c|c|c|c|}
\hline$\left[\mathbf{m}_{\text {styrene }} / \mathbf{m}_{\text {seed }}\right]$ & [mg] & $\mathrm{H}_{2} \mathrm{O}$ & 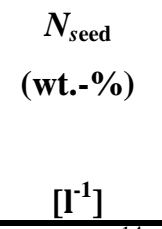 & [mg] & $\begin{array}{c}{[\mathrm{I}] /[\mathrm{M}]} \\
\text { ratio } \\
\\
{[\%]} \\
\end{array}$ & $\begin{array}{c}{[\mathbf{I}] /\left[\mathrm{H}_{2} \mathrm{O}\right]} \\
\text { ratio } \\
\\
{[\%]}\end{array}$ & $\begin{array}{c}\text { Diameter } \\
\text { SEM } \\
\text { (calculated)* } \\
\text { [nm] } \\
\end{array}$ \\
\hline 3 & 150 & 50 & $\begin{array}{c}2.65 \cdot 10^{14} \\
(0.1)\end{array}$ & 250 & 166.7 & 0.5 & $\begin{array}{c}241 \pm 21 \\
(238)\end{array}$ \\
\hline 5 & 250 & 50 & $\begin{array}{c}2.65 \cdot 10^{14} \\
(0.1)\end{array}$ & 250 & 100.0 & 0.5 & $\begin{array}{c}241 \pm 19 \\
(282)\end{array}$ \\
\hline 10 & 500 & 50 & $\begin{array}{c}2.65 \cdot 10^{14} \\
(0.1)\end{array}$ & 250 & 50.0 & 0.5 & $\begin{array}{c}255 \pm 37 \\
(355)\end{array}$ \\
\hline 20 & 1000 & 50 & $\begin{array}{c}2.65 \cdot 10^{14} \\
(0.1)\end{array}$ & 250 & 25.0 & 0.5 & $\begin{array}{c}483 \pm 68 \\
(448)\end{array}$ \\
\hline 30 & 1500 & 50 & $\begin{array}{c}2.65 \cdot 10^{14} \\
(0.1)\end{array}$ & 250 & 16.7 & 0.5 & $\begin{array}{c}550 \pm 41 \\
(513)\end{array}$ \\
\hline 40 & 2000 & 50 & $\begin{array}{c}2.65 \cdot 10^{14} \\
(0.1)\end{array}$ & 250 & 12.5 & 0.5 & $\begin{array}{c}488 \pm 28^{* *} \\
(564)\end{array}$ \\
\hline 50 & 2500 & 50 & $\begin{array}{c}2.65 \cdot 10^{14} \\
(0.1)\end{array}$ & 250 & 10.0 & 0.5 & $\begin{array}{l}\text { multimodal } \\
\text { (608) }\end{array}$ \\
\hline 100 & 5000 & 50 & $\begin{array}{c}2.65 \cdot 10^{14} \\
(0.1)\end{array}$ & 250 & 5.0 & 0.5 & $\begin{array}{l}\text { multimodal } \\
(766)\end{array}$ \\
\hline
\end{tabular}



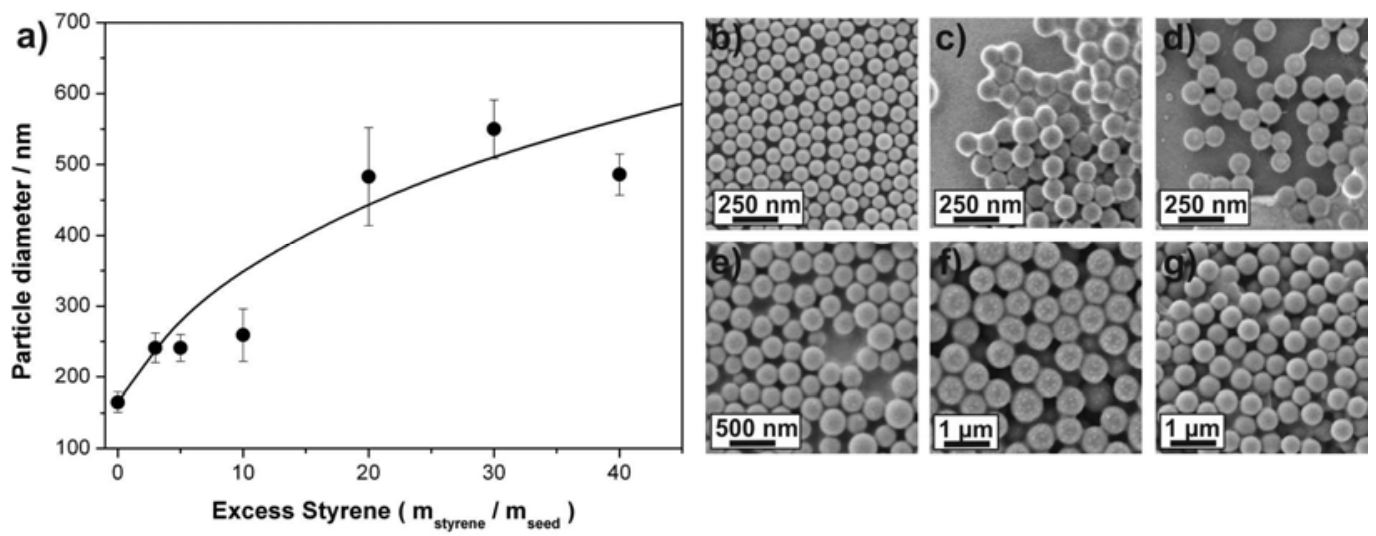

Figure 5.1.19. Variation of the amount of monomer added to control the resulting size inthe seeded emulsion polymerization process. The amount of styrene added is given as multiples of the total mass of the seed particles. (a) Dependency of the amount of styrene added on the resulting size. The line shown in the diagram indicates the theoretical size of the resulting particles assuming 100\% conversion of the additional monomer added. (b-g) Scanning electron microscopy images of the particles produced. (b) Seed particles as produced by a miniemulsion process (c) 3 fold excess of styrene. (d) 5 fold excess. (e) 10 fold excess. (f) 30 fold excess. (g) 40 fold excess.

The line shown in the diagram of Figure 5.1.19a indicates the theoretical size of the colloids assuming $100 \%$ conversion of the monomer in the seed particles. The diameters of the colloids produced in the set of experiments follow the relation loosely until a monomer excess of approximately 30 . With higher amounts of monomer added, secondary particles appear, leading to a reduction in size of the primary, seeded particles. Even higher monomer amounts lead to multimodal size distributions indicating uncontrolled reactions with secondary nucleation as the primary reaction pathway. ${ }^{[198]}$ Scanning electron microscopy (SEM) images of the different particles produced are shown in Figure 5.1.19b to g. Compared to the seed particles (Figure 5.1.19b), the size increased particles feature a higher homogeneity. This results from the uniform increase in diameter for all particles during the polymerization that leads to a reduction of the relative polydispersity. From the set of experiments, it can be stated that particles with a diameter up to at least $500 \mathrm{~nm}$ can be synthesized in a single reaction, a number that relates to an almost 40 fold increase in volume. Bigger sizes of the colloids are not trivially achieved as the standard reaction pathway breaks down with higher monomer excesses. Secondary nucleation or instable dispersions are the result.

As all reactions produced size increased particles, it can be stated that the initiator concentration in the aqueous phase was high enough to provide enough radicals for a successful polymerization. However, with the initiator concentration being constant in the aqueous phase, we face the undesirable situation to have an enormous amount of initiator with respect to the monomer added for small monomer excesses. Hence, the degree of polymerization is low and oligomers are formed that reside in the aqueous phase. These oligomers are seen in the SEM 
images in Figure 5.1.19c, d as amorphous material covering the colloidal particles. In the following chapter, the influence of the initiator concentration is discussed in more detail.

\subsection{Variation of the initiator to monomer ratio [I]/[M]}

Next, the influence of the initiator concentration on the outcome of the reaction was investigated. In contrary to normal bulk polymerizations, not only the amount of initiator relative to the monomer is important, but also the concentration in the water phase has to be taken into account as initiation and the first propagation steps take place in the continuous phase. Therefore, amounts of initiator needed are generally higher than in bulk polymerizations. Figure 5.1.20 presents the dependency of the initiator concentration on the resulting particle sizes, exemplarily for styrene excesses of 5 (Figure 5.1.20a) and 20 (Figure 5.1.20b). The parameters used for the reactions are summarized in Table 5.1.8. Dotted lines are inserted into the diagrams to indicate the original size of the seed particles as well as the theoretical sizes. Both sets of reactions show that lower initiator amounts (1-5 wt.- $\%$ with respect to the monomer added) do not induce an increase in size as the concentration of initiator in the water phase is insufficient to induce polymerization processes. The optimum initiator concentration for the seeded reactions as determined for 5- and 20fold excess of monomer can be estimated to be between 10 and $25 \mathrm{wt} .-\%$ of monomer. At higher amounts of initiator, the resulting particle sizes decrease (Figure 5.1.20a) as a result of an insufficient degree of polymerization that leads to oligomers formed that remain in the water phase as seen by the films covering the particles in the SEM images. As shown in the previous experiments, the most suitable values for the initiator concentration are not solely determined by the amount of monomer added but also by the concentration of radicals in the water phase. Hence, it can be expected that for higher monomer excesses, smaller values for the initiator to monomer ratio will be sufficient to induce polymerization. 
a)

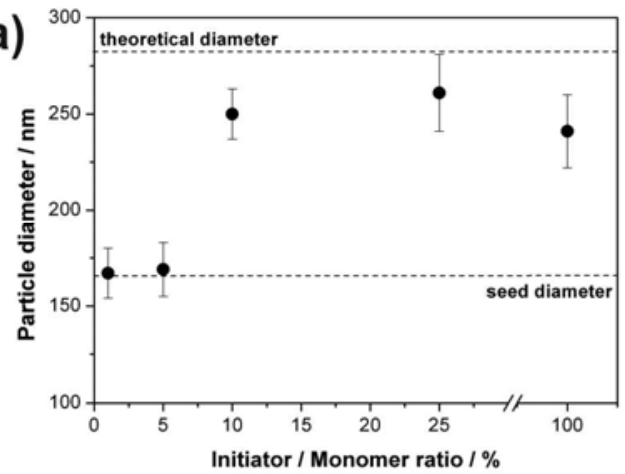

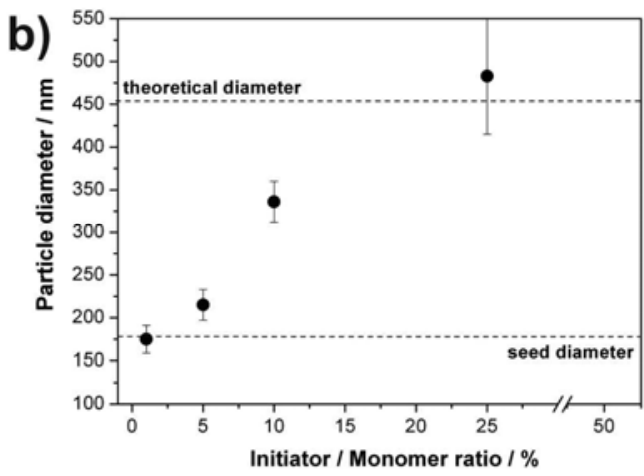

Figure 5.1.20. Variation of the amount of initiator relative to the amount of monomer added. The dotted lines are guides for the eyes and represent the diameter of the seed particles used in the reaction as well as the theoretical diameter reached for $100 \%$ conversion of the additional monomer added. (a) Reactions with 5 fold excess of styrene with respect to the mass of seed particles. (b) Reactions with 20 fold excess of styrene with respect to the mass of seed particles.

Table 5.1.8. Reaction details for the set of experiments shown in Figure 5.1.20. All reactions were carried out with $0.1 \mathrm{wt.}$ - $\%$ dispersions of seed particles.

\begin{tabular}{|c|c|c|c|c|c|c|c|}
\hline \multicolumn{2}{|c|}{ Styrene added } & \multirow{2}{*}{$\begin{array}{r}\mathrm{H}_{2} \mathrm{O} \\
\\
\\
/ \mathrm{g}\end{array}$} & \multirow{2}{*}{$\begin{array}{c}\mathrm{N}_{\text {seed }} \\
\text { (wt.-\%) } \\
/^{-1}\end{array}$} & \multirow{2}{*}{$\begin{array}{r}{[\mathrm{I}]} \\
/ \mathrm{mg}\end{array}$} & \multirow{2}{*}{$\begin{array}{c}{[\mathrm{I}] /[\mathrm{M}]} \\
\text { ratio } \\
\\
/ \%\end{array}$} & \multirow{2}{*}{$\begin{array}{c}{[\mathrm{I}] /\left[\mathrm{H}_{2} \mathrm{O}\right]} \\
\text { ratio } \\
\\
/ \%\end{array}$} & \multirow{2}{*}{$\begin{array}{c}\text { Diameter } \\
\text { SEM } \\
\text { (calculated*) } \\
\text { / nm } \\
\end{array}$} \\
\hline $\mathbf{m}_{\text {styrene }} / \mathbf{m}_{\text {seed }}$ & $/ \mathbf{m g}$ & & & & & & \\
\hline 5 & 250 & 50 & $\begin{array}{c}2.65 \cdot 10^{14} \\
(0.1)\end{array}$ & 2.5 & 1 & 0.005 & $\begin{array}{c}167 \pm 13 \\
(282)\end{array}$ \\
\hline 5 & 250 & 50 & $\begin{array}{c}2.65 \cdot 10^{14} \\
(0.1)\end{array}$ & 12.5 & 5 & 0.025 & $\begin{array}{c}169 \pm 14 \\
(282)\end{array}$ \\
\hline 5 & 250 & 50 & $\begin{array}{c}2.65 \cdot 10^{14} \\
(0.1)\end{array}$ & 25 & 10 & 0.05 & $\begin{array}{c}250 \pm 14 \\
(282)\end{array}$ \\
\hline 5 & 250 & 50 & $\begin{array}{c}2.65 \cdot 10^{14} \\
(0.1)\end{array}$ & 62.5 & 25 & 0.125 & $\begin{array}{c}261 \pm 20 \\
(282)\end{array}$ \\
\hline 5 & 250 & 50 & $\begin{array}{c}2.65 \cdot 10^{14} \\
(0.1)\end{array}$ & 250 & 100 & 0.5 & $\begin{array}{c}241 \pm 19 \\
(282)\end{array}$ \\
\hline 20 & 1000 & 50 & $\begin{array}{c}2.65 \cdot 10^{14} \\
(0.1)\end{array}$ & 10 & $\overline{1}$ & 0.02 & $\begin{array}{c}175 \pm 16 \\
(448)\end{array}$ \\
\hline 20 & 1000 & 50 & $\begin{array}{c}2.65 \cdot 10^{14} \\
(0.1)\end{array}$ & 50 & 5 & 0.1 & $\begin{array}{c}215 \pm 18 \\
(448)\end{array}$ \\
\hline 20 & 1000 & 50 & $\begin{array}{c}2.65 \cdot 10^{14} \\
(0.1)\end{array}$ & 100 & 10 & 0.2 & $\begin{array}{c}336 \pm 24 \\
(448)\end{array}$ \\
\hline 20 & 1000 & 50 & $\begin{array}{c}2.65 \cdot 10^{14} \\
(0.1)\end{array}$ & 250 & 25 & 0.5 & $\begin{array}{c}483 \pm 68 \\
(448)\end{array}$ \\
\hline
\end{tabular}

*calculated assuming $100 \%$ conversion of monomer to size-increased particles 


\subsection{Variation of type and concentration of surfactant}

Obviously, the type and amount of surfactant added to stabilize the seeded particles has a dramatic influence on the reaction pathway. ${ }^{[201]}$ This becomes clear when considering that the surfactant not only stabilizes the seed particles growing in size but can also adsorb to the formed particle nuclei. Hence, the surfactant adds to the stabilization of the nuclei, leading to a decrease in the number of nuclei that have to coagulate to form a stable secondary particle. It is expected that the amount of surfactant has to be limited to the least amount necessary to yield a stable emulsion. Figure 5.1.21 and Table 5.1.9 present the experimental data for reactions performed with varying surfactant types and amounts. As a test system, the reaction with a styrene excess of 100 was chosen as this led to multimodal size distributions in previous experiments (Figure 5.1.21 shows the result of the standard reaction presented in chapter 5.1.2.5.2). Obviously, the amount of SDS added in the standard recipe is insufficient to lead to stable reaction conditions. Hence, the SDS concentration in the continuous phase was increased from $0.01 \mathrm{wt} .-\%$ up to $0.1 \mathrm{wt} . \mathrm{\%}$. All SDS concentrations were below the $\mathrm{cmc}$ in order to avoid micelle formation that is used in conventional emulsion polymerizations. ${ }^{[28]}$ Figure $5.1 .21 \mathrm{~b}$ and $\mathrm{c}$ show representative SEM images of the resulting dispersions. For both cases, massive secondary nucleation took place, leading to bimodal size distributions. In the first case $(0.05 \mathrm{wt} .-\%$ SDS), the size increased seed particles feature an excellent monodispersity and have a diameter of approximately $600 \mathrm{~nm}$, indicating a more stable course of reaction. However, the presence of higher amounts of SDS stabilizes particle nuclei more efficiently and induces secondary nucleation. With a SDS concentration of $0.1 \mathrm{wt} .-\%$, almost all monomer added is converted into secondary particles, thus resembling a conventional emulsion polymerization. Only a minor fraction of size increased seed particles is found (Figure 5.1.21c). Changing the type of surfactant from the anionic SDS to the nonionic Lutensol AT50 compromised the reaction stability. As depicted in Figure 5.1.21d, the reaction did not lead to a successful conversion of monomer to size increased particles. Instead, the seed particles only marginally grew in size and most of the monomer added was found as a polymeric film covering the complete surface. This is not unexpected as nonionic surfactants are known to stabilize colloids less efficiently than charged surfactants. Thus, usually significantly larger amounts of nonionic surfactants are needed to stabilize droplets or particles.

Concluding these experiments it can be stated that the amount and type of surfactant crucially influences the reaction pathway. Surfactant has to be added in order to stabilize the growing particles; however, the amount has to be as small as possible. Otherwise, secondary nucleation becomes the primary reaction pathway. Therefore, the direct conversion of small seed particles to very big sizes above approximately $600 \mathrm{~nm}$ was impeded. From a technological point of 
view, seeded particles with bigger sizes have to be synthesized in a step-wise way using consecutive seeding reactions. As an alternative, the secondary particles appearing from the reaction with 0.05 wt.-\% SDS were also successfully removed by centrifugation.
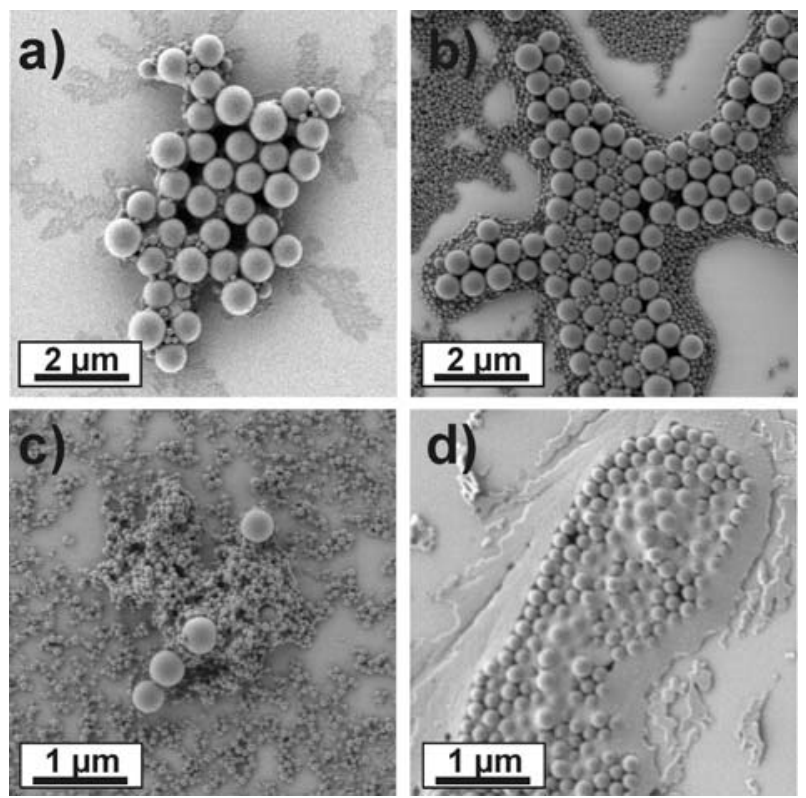

Figure 5.1.21. Effect of different concentrations and types of surfactants applied to stabilize the particles in the process. All concentrations given are relative to the weight of the water phase. (a) $0.01 \mathrm{wt} .-\%$ SDS.

(b) 0.05 wt.- $\%$ SDS. (c) 0.1 wt.-\% SDS. (d) 0.1 wt.- $\%$ Lutensol AT50.

Table 5.1.9. Reaction details for the set of experiments shown in Figure 5.1.21. All reactions were carried out with $0.1 \mathrm{wt} .-\%$ dispersions of seed particles.

\begin{tabular}{|c|c|c|c|c|c|c|}
\hline $\begin{array}{c}\text { Sample } \\
\text { in }\end{array}$ & Styrene added & $\mathrm{H}_{2} \mathrm{O}$ & $N_{\text {seed }}$ & [I] & surfactant & $\begin{array}{c}\text { surfactant } \\
\text { conc. }\end{array}$ \\
\hline
\end{tabular}

Figure

5.1.21

$\left[m_{\text {styrene }} / m_{\text {seed }}\right] \quad[\mathrm{mg}] \quad[\mathrm{g}] \quad\left[\mathrm{l}^{-1}\right] \quad[\mathrm{mg}] \quad[\mathrm{wt}$. \% of

\begin{tabular}{cccccccc}
\hline a) & 100 & 250 & 50 & $\begin{array}{c}2.65 \cdot 10^{14} \\
(0.1)\end{array}$ & 250 & SDS & 0.01 \\
& & & & & & $\left.\mathbf{H}_{2} \mathbf{O}\right]$ \\
b) & 100 & 250 & 50 & $2.65 \cdot 10^{14}$ & 250 & SDS & 0.05 \\
& & & & $(0.1)$ & & & $(25 \mathrm{mg})$ \\
c) & 100 & 250 & 50 & $2.65 \cdot 10^{14}$ & 250 & SDS & 0.1 \\
& & & & $(0.1)$ & & & $(50 \mathrm{mg})$ \\
d) & 100 & 250 & 50 & $2.65 \cdot 10^{14}$ & 250 & Lutensol & 0.1 \\
& & & & $(0.1)$ & & AT50 & $(50 \mathrm{mg})$ \\
\hline
\end{tabular}




\subsection{Variation of continuous phase composition}

The continuous phase has an important influence on the reaction pathway as well. The monomer added has to diffuse through the continuous phase in order to reach the seed particles. Initiation takes place in the continuous phase. Additionally, the continuous phase also influences both stabilization of seed particles and particle nuclei forming secondary particles in the course of the reaction. Furthermore, diffusion and solubility of the monomer is affected drastically by changes in the continuous phase. In order to investigate these effects, systematic variations of the continuous phase were performed. Figure 5.1.22 and Table 5.1.10 show the properties of the resulting dispersions and the reaction details respectively.

First, sodium chloride was added to the continuous phase. The presence of salt affects the stability of electrostatically stabilized colloidal particles as the electrical double layer at the colloid interface is screened by the counter ions. Therefore, the presence of salt might induce changes to the reaction pathway. Once particle nuclei are formed by coil-to-globule transitions of oligoradicals, these nuclei will tend to assemble with other nuclei to form a stable secondary particle. As the electrostatic repulsion is screened, more particle nuclei are needed to induce stability of the secondary particle. Hence, the agglomeration of the particle nuclei to the seed particles should be favored. The experimental data (Figure 5.1.22a) seems to support this model. While very small amounts of sodium chloride ( 0.05 wt.-\% with respect to the water phase) do not affect the polymerization and the final particle diameter essentially resembles the reaction without salt, the addition of 0.1 wt.- $\%$ of $\mathrm{NaCl}$ induces an increase in final particle size to very close to the theoretical value. Larger amounts of $\mathrm{NaCl}$ interfere with electrostatic stabilization and lead to irregularly shaped particles and partial aggregation as the stability of the seed particles starts to be compromised by screening effects.

Next, ethanol was added to the continuous phase. In that case, the stability of the latex particles is not crucially affected as only a maximum of $20 \mathrm{wt} .-\%$ of ethanol was added. It was expected that ethanol addition will increase the solubility of styrene in the continuous phase. The oligoradical formed will have a different solubility in ethanol-containing water as well. Thus, the balance between the two reaction pathways (oligoradical entering a seed particle or collapsing) will be affected by a change of the composition of the continuous phase.

The experimental results, shown in Figure 5.1.22b show the following characteristics. First, small amounts of ethanol (10 wt.- \%) induce well-growing seed particles that feature a final size close to the theoretical expected value. A minor amount of secondary particles is visible as observed by electron microscopy. Increasing the ethanol content induces a drop in final diameter close to the value measured for particles synthesized in pure water. Additionally, the 
amount of formed secondary particles drastically increases. It is worth mentioning that the particles from reactions with ethanol generally feature a higher monodispersity.

Finally, methanol was added to the continuous phase as well (Figure 5.1.22c). Surprisingly, no influence on the reaction was detected, even for a methanol content of $20 \mathrm{wt} . \mathrm{\%}$ and all dispersions produced closely resemble each other in terms of final size and homogeneity.

Table 5.1.10. Reaction details for the set of experiments shown in Figure 5.1.22. All reactions were carried out with 0.1 wt.- $\%$ dispersions of seed particles.

\begin{tabular}{|c|c|c|c|c|c|c|c|}
\hline \multirow[b]{2}{*}[\mathbf{m}_{\text{styrene}}/\mathbf{m}_{\text{seed}}]{} & \multirow[b]{2}{*}{ [mg] } & \multirow{2}{*}{$\begin{array}{r}\mathrm{H}_{2} \mathrm{O} \\
\\
{[\mathrm{g}]}\end{array}$} & \multirow{2}{*}{$\begin{array}{c}\mathbf{N}_{\text {seed }} \\
\text { (wt.-\%) } \\
{\left[\mathrm{l}^{-1}\right]}\end{array}$} & \multirow{2}{*}{$\begin{array}{c}\text { [I] } \\
{[\mathrm{mg}]}\end{array}$} & \multirow{2}{*}{$\begin{array}{c}\text { SDS } \\
\text { conc. } \\
\text { [wt.-\% } \\
\text { of } \\
\left.\mathrm{H}_{2} \mathrm{O}\right] \\
\end{array}$} & \multirow{2}{*}{$\begin{array}{c}\text { Addition } \\
\text { Cont. Phase }\end{array}$} & \multirow{2}{*}{$\begin{array}{c}\text { Diameter } \\
\text { SEM } \\
\text { (calculated }^{*} \text { ) } \\
\text { [nm] }\end{array}$} \\
\hline & & & & & & & \\
\hline 20 & 1000 & 50 & $\begin{array}{c}2.65 \cdot 10^{14} \\
(0.1)\end{array}$ & 100 & $\begin{array}{c}0.01 \\
(5 \mathrm{mg})\end{array}$ & $\overline{---}$ & $\begin{array}{c}336 \pm 24 \\
(448)\end{array}$ \\
\hline 20 & 1000 & 50 & $\begin{array}{c}2.65 \cdot 10^{14} \\
(0.1)\end{array}$ & 100 & $\begin{array}{c}0.01 \\
(5 \mathrm{mg})\end{array}$ & $\begin{array}{c}60 \mathrm{mg} \mathrm{NaCl} \\
\left(0.05 \mathrm{~mol} \cdot \mathrm{l}^{-1}\right)\end{array}$ & $\begin{array}{c}323 \pm 35 \\
(448)\end{array}$ \\
\hline 20 & 1000 & 50 & $\begin{array}{c}2.65 \cdot 10^{14} \\
(0.1)\end{array}$ & 100 & $\begin{array}{c}0.01 \\
(5 \mathrm{mg})\end{array}$ & $\begin{array}{c}120 \mathrm{mg} \mathrm{NaCl} \\
\left(0.1 \mathrm{~mol} \cdot \mathrm{l}^{-1}\right)\end{array}$ & $\begin{array}{c}427 \pm 26 \\
(448)\end{array}$ \\
\hline 20 & 1000 & 50 & $\begin{array}{c}2.65 \cdot 10^{14} \\
(0.1)\end{array}$ & 100 & $\begin{array}{c}0.01 \\
(5 \mathrm{mg})\end{array}$ & $\begin{array}{c}300 \mathrm{mg} \mathrm{NaCl} \\
\left(0.5 \mathrm{~mol} \cdot \mathrm{l}^{-1}\right)\end{array}$ & $\begin{array}{c}330 \pm 28 \\
(448)\end{array}$ \\
\hline 20 & 1000 & 50 & $\begin{array}{c}2.65 \cdot 10^{14} \\
(0.1)\end{array}$ & 100 & $\begin{array}{c}0.01 \\
(5 \mathrm{mg})\end{array}$ & $\begin{array}{l}600 \mathrm{mg} \mathrm{NaCl} \\
\left(0.5 \mathrm{~mol} \cdot \mathrm{l}^{-1}\right)\end{array}$ & $\begin{array}{l}\text { irreg. shapes } \\
\text { (448) }\end{array}$ \\
\hline 20 & 1000 & 45 & $\begin{array}{c}2.65 \cdot 10^{14} \\
(0.1)\end{array}$ & 100 & $\begin{array}{c}0.01 \\
(5 \mathrm{mg})\end{array}$ & $\begin{array}{l}5 \mathrm{mg} \mathrm{EtOH} \\
(10 \text { wt.-\%) }\end{array}$ & $\begin{array}{c}463 \pm 25^{* *} \\
(448)\end{array}$ \\
\hline 20 & 1000 & 40 & $\begin{array}{c}2.65 \cdot 10^{14} \\
(0.1)\end{array}$ & 100 & $\begin{array}{c}0.01 \\
(5 \mathrm{mg})\end{array}$ & $\begin{array}{c}10 \mathrm{mg}(\mathrm{EtOH}) \\
(20 \%)\end{array}$ & $\begin{array}{c}377 \pm 26^{* *} \\
(448)\end{array}$ \\
\hline 20 & 1000 & 45 & $\begin{array}{c}2.65 \cdot 10^{14} \\
(0.1)\end{array}$ & 100 & $\begin{array}{c}0.01 \\
(5 \mathrm{mg})\end{array}$ & $\begin{array}{c}5 \mathrm{mg} \mathrm{MeOH} \\
(10 \%)\end{array}$ & $\begin{array}{c}330 \pm 27 \\
(448)\end{array}$ \\
\hline 20 & 1000 & 40 & $\begin{array}{c}2.65 \cdot 10^{14} \\
(0.1)\end{array}$ & 100 & $\begin{array}{c}0.01 \\
(5 \mathrm{mg})\end{array}$ & $\begin{array}{c}10 \mathrm{mg} \mathrm{MeOH} \\
(20 \%)\end{array}$ & $\begin{array}{c}355 \pm 27 \\
(448)\end{array}$ \\
\hline
\end{tabular}

*calculated assuming $100 \%$ conversion of monomer to size-increased particles *secondary particle formation observed 

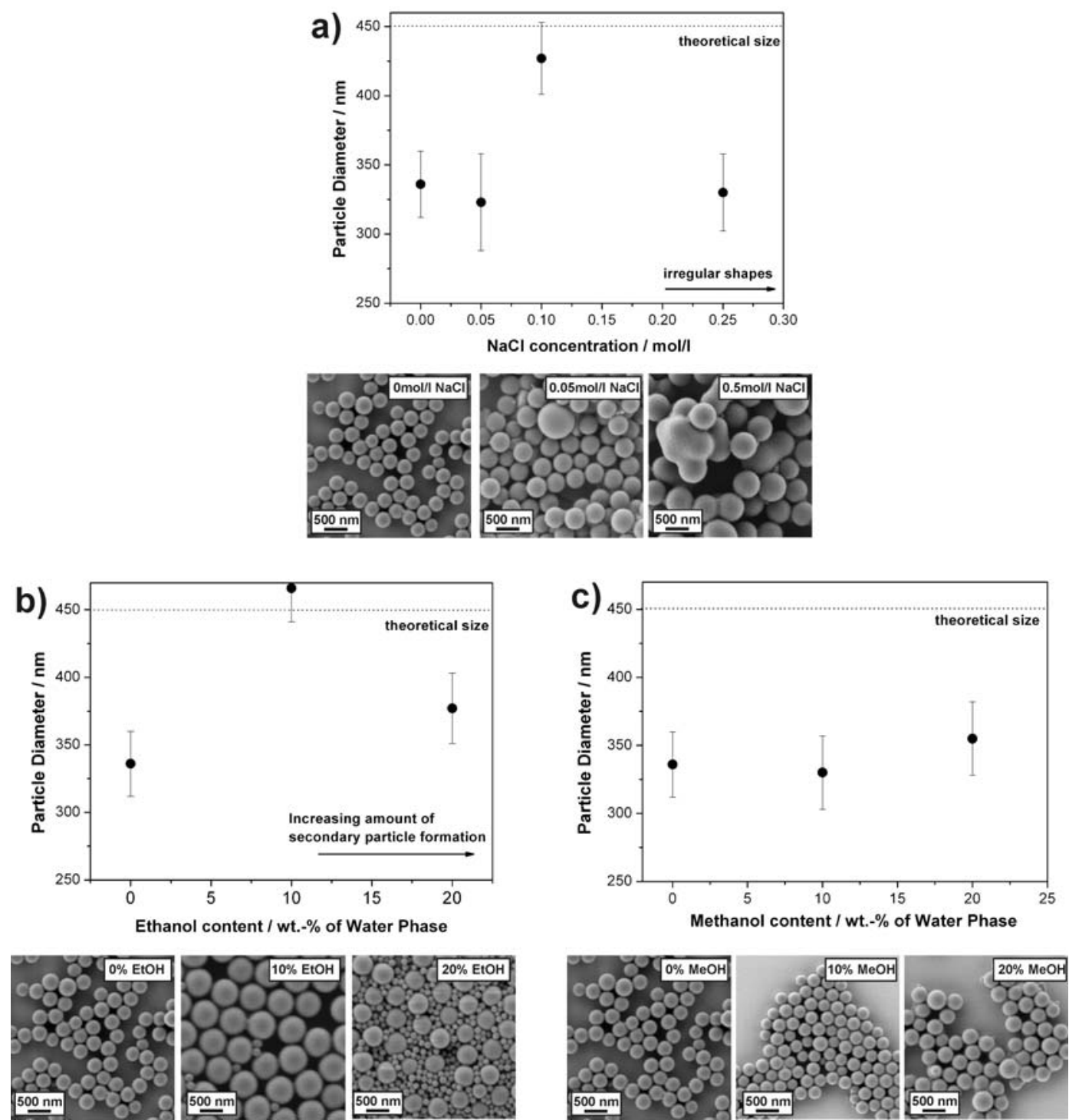

Figure 5.1.22. Effects of the composition of the continuous phase on the seeded polymerization reactions. The dotted lines in the spectra represent the theoretical size of the colloids assuming $100 \%$ conversion of the monomer added. Representative scanning electron microscopy images are shown below the different sprectra. (a) Addition of sodium chloride to the continuous water phase. (b) Addition of ethanol to the water phase. For reasons of clarity, only seeded particles were analyzed. Secondary particles are not shown in the diagram. c) Addition of methanol to the continuous water phase.

\subsection{Conclusions}

The following conclusions regarding experimental conditions of the seeded emulsion polymerization reactions can be drawn from the experiments performed. i) It is possible to synthesize functional colloidal particles with a diameter up to $600 \mathrm{~nm}$ in one step. Larger diameters have to be synthesized in a step-by-step fashion. ii) The initiator concentration has to be high in order to induce polymerization and not only depends on the amount of monomer 
added, but also on the amount of the continuous phase. Best results were achieved using 1025 wt.-\% APS with respect to the amount of monomer added. iii) Choice and concentration of surfactant is very critical for a successful reaction. SDS as an anionic surfactant proved to be superior to Lutensol AT50 as nonionic surfactant. High surfactant concentrations lead to more homogeneous particles but eventually favor secondary nucleation as the primary reaction pathway. Therefore, the surfactant concentration ought to be as small as possible in order to stabilize the dispersion without inducing secondary nucleation. iv) The composition of the continuous phase influences the reaction as well. Small amounts of salt lead to final particle sizes reaching the theoretical diameter while higher salt amounts compromise the stability of the dispersion. Ethanol might be added to obtain very monodisperse samples; however, care has to be taken as secondary nucleation seems to be favored as well.

\subsection{Precise adjustment of the colloid size for Pt(acac) $)_{2}$ containing particles to be used in the non-conventional lithography process}

For experiments on the non-conventional lithography approach that will be presented in chapter 5.3, a systematic increase in particle size with small steps of only several nanometers was desired to investigate the precision in interparticle distance of the final platinum nanoparticle arrays. Platinum(II)acetylacetonate containing seed particles were prepared by a surfactant-free emulsion polymerization approach as described in chapter 5.1.2.2.1 in order to obtain a reasonably narrow size distribution. These particles were consequently subjected to a seeded emulsion polymerization with little amounts of styrene added to systematically increase the diameter. As seed particles, polystyrene colloids with a mean diameter of $132 \mathrm{~nm}$, containing 1.25 wt.- $\%$ platinum, were prepared (Table 5.1.11). The encapsulation efficiency was $66.1 \%$, in agreement with values found for similar incorporation reactions with $\mathrm{Pt}(\mathrm{acac})_{2}$ (chapter 5.1.2.2.1). The colloids were used as seed particles in order to adjust their sizes without changing the platinum content. Table 5.1.11 summarizes the experimental details and the characteristics of the resulting particles. Figure 5.1.23 presents the dependence of the particle diameter on the amount of styrene added. The relative quantity styrene excess $\left([M] /\left[M_{0}\right]\right)$ was used to characterize the mass of styrene added in relation to the total mass of the seed particles. The absolute numbers of the mass of styrene and initiator added are given in Table 5.1.11. 

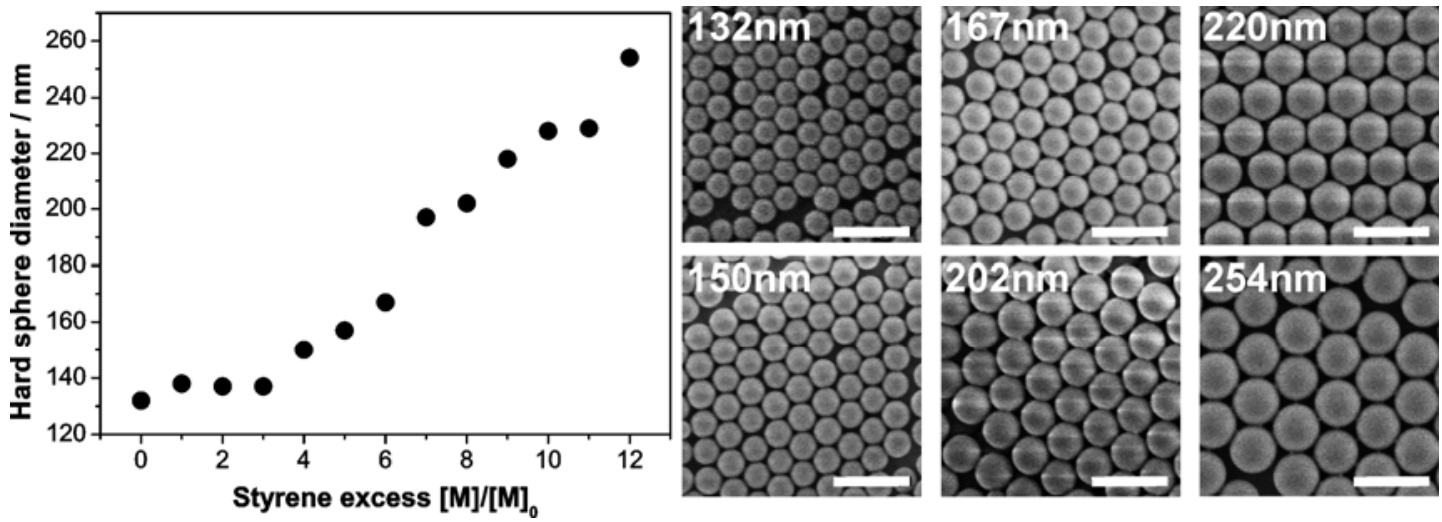

Figure 5.1.23. Seeded emulsion polymerization of $132 \mathrm{~nm}$ platinum containing seed particles. Left side: diagram showing the diameter of the seed particles in dependence on the amount of styrene added. The right side shows a selection of SEM images of the final, size increased particles. The scale bar is $400 \mathrm{~nm}$.

Table 5.1.11. Summary of the seeded polymerization of $132 \mathrm{~nm} \mathrm{Pt}(\mathrm{acac})_{2}$-containing particles and characterization of size and platinum content of the resulting colloids.

\begin{tabular}{|c|c|c|c|c|c|c|c|c|}
\hline \multirow{2}{*}{\multicolumn{2}{|c|}{$\begin{array}{l}\text { Monomer added } \\
\text { Monomer } \\
\text { excess }\end{array}$}} & \multirow{2}{*}{$\begin{array}{c}\text { Initiator } \\
\text { [mg] } \\
\end{array}$} & \multicolumn{3}{|c|}{ Diameter } & \multicolumn{3}{|c|}{ Platinum content } \\
\hline & & & $\begin{array}{l}\text { Theory } \\
\text { [nm] }\end{array}$ & $\begin{array}{l}\text { SEM } \\
\text { [nm] }\end{array}$ & $\begin{array}{l}\text { PDI* } \\
\text { [\%] }\end{array}$ & $\begin{array}{l}\% \text { of } \\
\text { seed } \\
{[\%]}\end{array}$ & $\begin{array}{c}\text { \%of } \\
\text { theory** } \\
{[\%]}\end{array}$ & [wt.-\%] \\
\hline 0 (seed) & --- & --- & 132 & $132 \pm 6$ & 4.5 & 100.00 & 100.0 & 1.26 \\
\hline 1 & 125 & 12.5 & 149 & $138 \pm 6$ & 4.3 & 94.21 & 107.2 & 1.18 \\
\hline 2 & 250 & 25 & 178 & $137 \pm 5$ & 3.6 & 92.43 & 102.9 & 1.16 \\
\hline 3 & 375 & 37.5 & 199 & $137 \pm 5$ & 3.6 & 94.36 & 105.1 & 1.18 \\
\hline 4 & 500 & 50.0 & 217 & $150 \pm 5$ & 3.3 & 64.94 & 94.9 & 0.81 \\
\hline 5 & 625 & 62.5 & 232 & $157 \pm 7$ & 4.5 & 60.43 & 101.3 & 0.76 \\
\hline 6 & 750 & 75.5 & 246 & $193 \pm 6$ & 3.1 & 41.68 & 103.5 & 0.42 \\
\hline 7 & 875 & 87.5 & 256 & $197 \pm 11$ & 5.6 & 26.38 & 87.3 & 0.33 \\
\hline 8 & 1000 & 100.0 & 269 & $202 \pm 8$ & 4.0 & 31.81 & 113.6 & 0.38 \\
\hline 9 & 1125 & 112.5 & 279 & $218 \pm 6$ & 2.8 & 20.51 & 92.0 & 0.32 \\
\hline 10 & 1250 & 125.0 & 288 & $228 \pm 7$ & 3.1 & 19.31 & 99.1 & 0.24 \\
\hline 11 & 1375 & 137.5 & 297 & $229 \pm 9$ & 3.9 & 20.84 & 108.4 & 0.27 \\
\hline 12 & 1500 & 150.0 & 306 & $254 \pm 10$ & 3.9 & 15.76 & 111.9 & 0.16 \\
\hline
\end{tabular}

*calculated from statistical analysis of SEM images.

**to calculate the theoretical amount of platinum for the seeded particles, the Pt content of the seed particles determined by ICP was set to $100 \%$; the actual encapsulation efficiency was $66.1 \%$.

A clear increase in particle diameter with increasing amount of styrene added was found. However, the diameter of the resulting seed particles was significantly lower than the theoretically predicted diameter (Table 5.1.11). Additionally, an increase in diameter is detected only for a styrene excess of 4 or higher. It is speculated that the discrepancy between the theoretically expected, calculated diameter and resulting particle size is based on the small reaction volumes used for the seeding experiments. More precisely, the loss of monomer is 
attributed to the following points. i) Both water and air phase of the sealed reaction vessel are saturated with monomer that is thus not available for the reaction. ii) Formation of water-soluble oligomers can be expected by termination reactions. However, as can be seen in Figure 5.1.23 the diameter of the resulting seeded colloids can be adjusted within several nanometers, thus giving access to a precise investigation of the resulting interparticle distances in the nonconventional lithography process that will be presented in chapter 0 .

No secondary nucleation occurred as was detected by electron microscopy investigations (Figure 5.1.23). The results of the metal content analysis are presented in Figure 5.1.24. The left diagram presented the measured metal contents in percent of the theoretical amount calculated for the diameter of the seeded particles. No loss of platinum was detected throughout the set of prepared particles and the measured values are close to the values predicted theoretically. This indicates that the platinum complex molecules remain entrapped in the seed particle during the reaction. Additionally, the results suggest that no secondary particles were formed. If such particles were present in the dispersion, they would contribute to the solid content used for the calculation of the theoretical amount of platinum. Because secondary particles do not contain any platinum, the metal content determined experimentally would be lowered with respect to the theoretical content. The right diagram shows the same experimental data presented in percent of the platinum content of the seed particle. The relative amount of platinum decreases with increasing mass of the seed particle $m_{\text {seed }}$ relative to the mass of the initial particle $m_{\text {ini }}$; or with a $\mathrm{d}^{-3}$-dependency with respect to the diameter $d_{\text {seed }}$ of the size-increased particle relative to the diameter $d_{i n i}$ of the initial particle:

$$
P t[\%]=100 \% \cdot \frac{m_{\text {ini }}}{m_{\text {seed }}}=100 \% \cdot \frac{d_{\text {ini }}^{3}}{d_{\text {seed }}^{3}}
$$

The theoretical platinum content is shown as a broken line in Figure 5.1.24b. The data points closely follow this line, indicating that the platinum content decreases precisely with increasing mass of the particles. This demonstrates that the platinum complexes are indeed entrapped in the seed particles and do not diffuse out of the colloid during the reaction. 

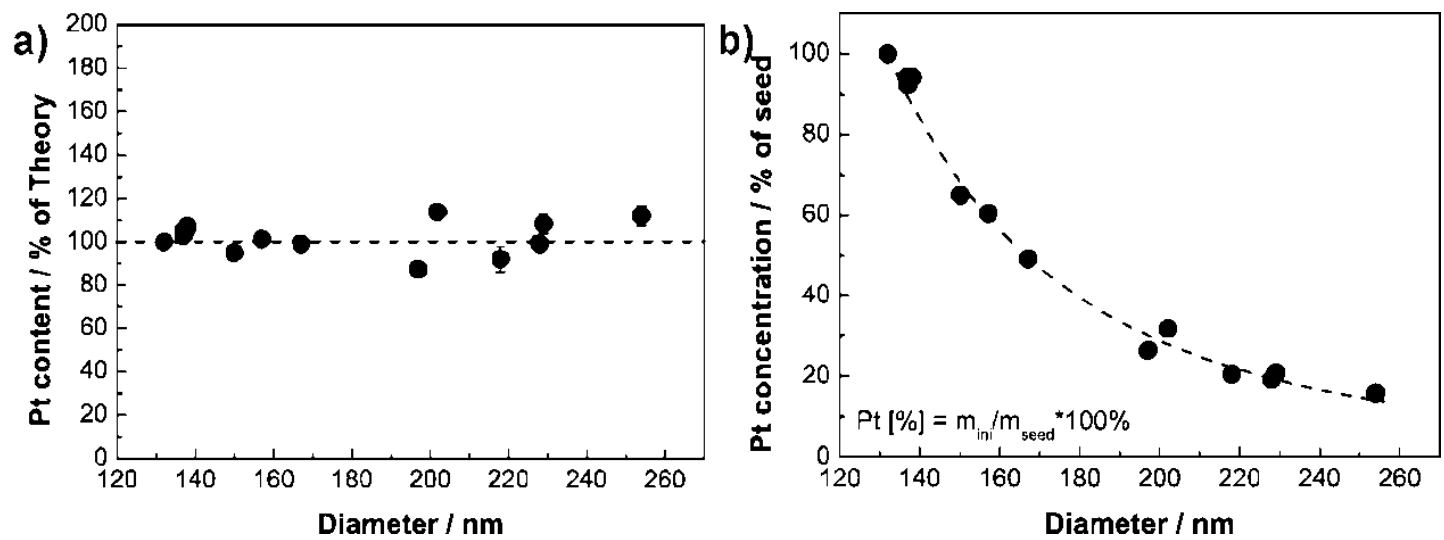

Figure 5.1.24. ICP-OES analysis of the prepared seeded particles. a) Platinum content in percent of the theoretical value plotted against the diameter of the particles The line represents the theoretical value. $b$ ) Platinum content in \% of the seed particle plotted against the diameter of the colloids. The dotted line represents the theoretical value calculated by the increase in mass of the seeded particles.

Summarizing, it can be stated that the diameter of platinum-acetylacetonate containing particles can be adjusted by a seeded emulsion polymerization and that no loss of the platinum complex occurred during the reaction. Additionally, no secondary nucleation was observed, thus guaranteeing a homogenous distribution of platinum among the latex particles 


\subsection{Assembly of colloidal monolayer architectures}

In this chapter, approaches for the two dimensional crystallization of colloidal latex particles are presented.

First, a method is described for the convenient assembly of single-sized particles into closepacked monolayers. Although a variety of crystallization processes are known in literature (chapter 3.3.1), the method introduced here substantially reduces time and experimental efforts while still yielding highly ordered two dimensional crystals over large areas and on a variety of substrates with arbitrary topographies.

Close-packed monolayers were subjected to a plasma treatment process to reduce the size of individual particles while keeping the lattice spacing constant. In this way, arrays of non-closepacked monolayers are created that reflect the high order and symmetry of the parental, closepacked monolayer. A detailed examination on the effects of etching conditions as well as material aspects of the substrate and colloids on the etching rates and the morphology of the monolayer is presented. UV/Vis spectroscopy is used to investigate the optical features of the monolayer that are highly sensitive to colloid size and packing density. A simple model based on the geometric arrangement of the monolayer is derived to explain the coloration.

Finally, more complex monolayer architectures are presented. Binary monolayer architectures are produced using a co-crystallization process at the air water interface on a Langmuir trough. Adjustment of the stoichiometry of the two particle types at the air/water interface leads to a high degree of control over the occupation of the interstitial sites in the close-packed layer of large spheres by the small colloids. Thus, large areas of binary $2 \mathrm{D}$ crystals with $\mathrm{LS}_{2}, \mathrm{LS}_{6}$ and $\mathrm{LS}_{9}$ structures are fabricated in a controlled way. The process allows the formation of binary crystals over a wide range of particle size ratios from 0.19 to 0.40 . The results are published in two articles entitled "A Convenient Method to Produce Close-packed and Non-close-packed Colloidal Monolayers Using Direct Assembly at the Air/Water Interface Followed by Plasma Induced Size Reduction",[94] and "Wafer-Scale Fabrication of Ordered Binary Colloidal Monolayers with Adjustable Stoichiometries". ${ }^{73]}$ 


\subsubsection{Convenient production of close-packed monolayers using direct assembly at the air/water interface}

\subsubsection{Introduction}

A number of different crystallization processes for the construction of colloidal monolayers have been described in literature and are critically discussed in chapter 3.3. From a conceptual point of view, the ideal preparation process reliably yields highly symmetric assemblies and prevents multi-layer structures or incomplete arrangements. Additionally, it is fast, experimentally simple and able to produce large area crystals. As already explained in chapter 3.3.1, the crystallization methods can be divided into two subcategories: direct assembly processes and interface-mediated methods. The latter features several inherent advantages. Most importantly, the interface guarantees a two-dimensional assembly and avoids multilayer formation. Additionally, at a liquid interface, the particles retain a high mobility that allows them to assemble into the minimum free energy position in a hexagonal lattice. The predominant assembly techniques that use a liquid interface are conducted on Langmuir troughs. ${ }^{[72,80,88]}$ Though being able to produce monolayers of large areas and exceptional high quality, the method's drawbacks are rather long preparation times and the requirement of special instruments (i.e. the Langmuir trough).

In this chapter, the existing Langmuir trough-based technique is substantially simplified to produce high quality monolayers within minutes and without the use of professional equipment. The process presented here uses a direct assembly of colloids at the air/water interface in a way that no additional compression of the monolayer is necessary prior transfer. The close-packed monolayer directly forms at the air/water interface and can be transferred to arbitrary substrates simply by immersion of the substrate into the water phase and picking up the monolayer with a transfer substrate of arbitrary material or topography.

\subsubsection{Results and Discussion}

Figure 5.2.1a to e shows the process flow for the monolayer crystallization at the air/water interface and the subsequent transfer to a solid substrate. The colloidal dispersion was diluted with $50 \mathrm{wt.}$ - $\%$ ethanol to facilitate spreading. Addition to the air/water interface was performed 
via a glass slide tilted with an angle of approximately $45^{\circ}$ (a). Upon spreading, patches of colloidal monolayers formed and remained afloat at the interface $(b, c)$. The monolayer patches continued to grow in size upon further addition of colloids until the complete interface was covered with a monolayer. Further addition of colloids led to monolayer buckling (i.e. the formation of multilayer structures at the interface) and increasing submersion of particles into the subphase thus compromising the quality of the monolayer. Therefore, care was taken to stop the colloid addition before the complete interface was covered. A solid substrate was immersed into the water phase (d) and elevated at a shallow angle through the interface in order to collect the monolayer (e). Drying was performed at a tilted angle of approximately $45^{\circ}$. Photographs of the process using $1 \mu \mathrm{m}$ colloids are shown in Figure 5.2.1f to i. Figure 5.2.1f shows the addition of the colloidal dispersion to the air/water interface via a tilted glass slide. Patches of ordered monolayers are already afloat on the interface as can be seen by the vivid colors that arises due to Bragg reflections of the different crystal domains. In Figure 1g, the transfer to a 2"-wafer is shown. The wafer is immersed into the subphase and lifted at a shallow angle to transfer the monolayer. The same wafer after drying is shown in i). A uniform coverage with colloids is visible. Individual crystal domains can be distinguished by their distinct coloration. The effect of adding too much colloid dispersion is shown in Figure 5.2.1h. The formation of multilayered, disordered structures is seen by the white color of the interface around the glass slide.
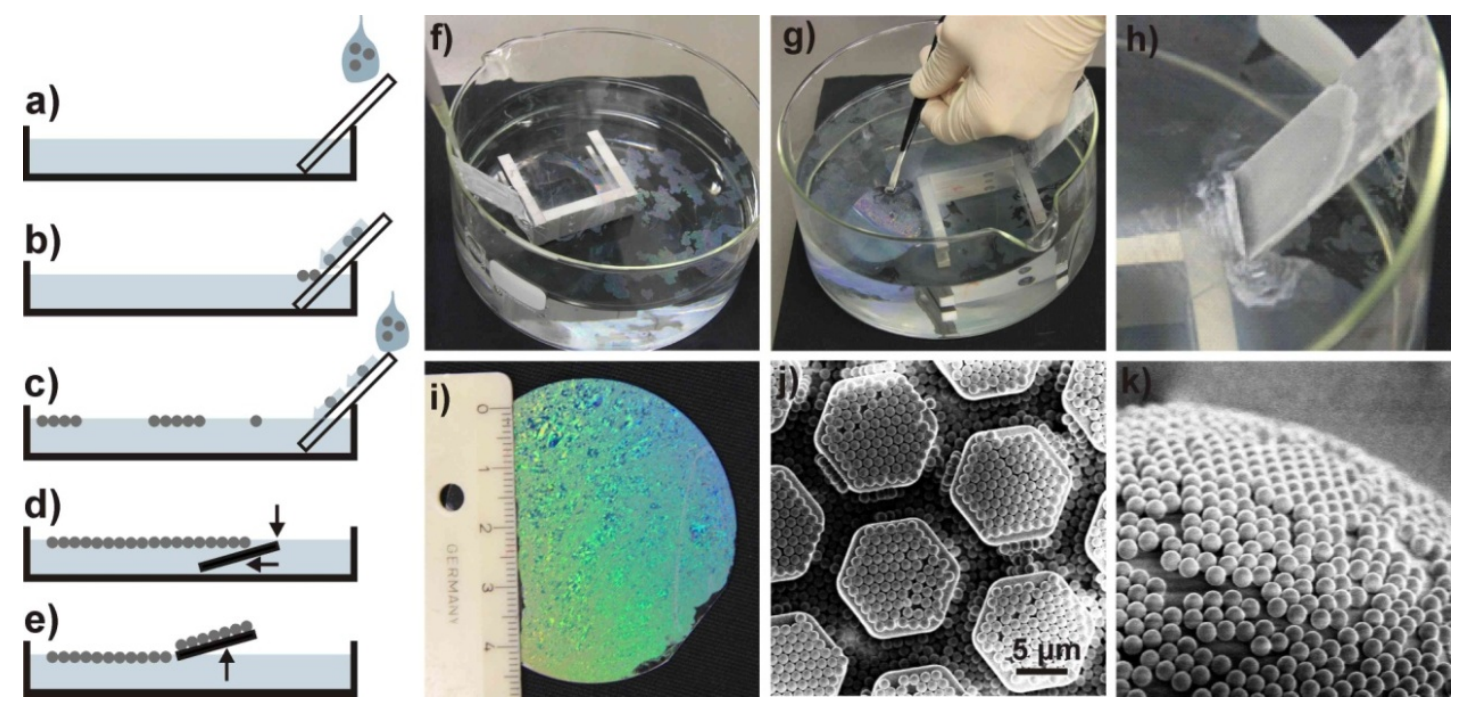

Figure 5.2.1. Schematic illustration and photographs of the monolayer fabrication process: a,b) Addition of colloids to the interface via a tilted glass slide; c) formation of a close-packed monolayer; d,e) monolayer transfer by immersion of the transfer substrate and subsequent elevation under a shallow angle; f) photograph of the deposition of $1 \mu \mathrm{m}$ colloids onto the air/water interface; individual, crystalline monolayer patches floating at the interface can be seen; g) photograph of the monolayer transfer onto a 2" wafer; h) the same wafer after drying; i) electron micrograph of a colloidal monolayer deposited onto arrays of micropillars produced by photolithography. 
The process is versatile with respect to colloidal size and substrate material. Polystyrene particles with diameters between 130 and $1000 \mathrm{~nm}$ were successfully applied in the process. Transfer to solid substrates was straightforward and conveniently done by hand. There are no special requirements for the substrate except for that it must be sufficiently hydrophilic in order not to completely dewet during the deposition process. However, even hydrophobic substrates can be used if they are treated with oxygen plasma for a short time prior to transfer. Planarity of the surface is generally not a requirement for the deposition process and substrates with arbitrary topographies have been covered by a similar transfer process. This is demonstrated in Figure 5.2.1j and $\mathrm{k}$ that present electron micrographs of a monolayer of $1 \mu \mathrm{m}$ colloids deposited onto an array of photoresist micropillars produced by conventional photolithography (j) and on top of $50 \mu \mathrm{m}$ polystyrene latex particles $(\mathrm{k})$.

The quality of the resulting monolayer is strongly affected by the composition of the continuous phase. Using pure water (milliQ grade) for the assembly led to the formation of a monolayer of colloids at the interface that did not possess a high degree of short- or long range order. Figure 5.2.2a presents a monolayer of $408 \mathrm{~nm}$ polystyrene colloids crystallized at a pure water interface. The low degree of order in the monolayer is visible. Two ways of increasing the order in the resulting monolayers were investigated. First, small amounts of sodium dodecyl sulfate (SDS) were added to the water subphase. As these molecules are amphiphilic, they will accumulate at the air/water interface and constitute a soft barrier for the colloids added to the interface. This barrier facilitates the packing process of the individual colloidal particles into a hexagonal lattice. On a molecular scale, the particles detaching from the glass slide at the interface are pushing against the floating surfactant layer which in turn pushes the particles closer together and thus facilitates the nucleation of two-dimensional crystals. This feature has been coined "piston oil effect" ${ }^{[55]}$ It has been shown that monolayers, which were floated in the presence of surfactants, exhibited a higher mechanical stability and fractured less easily at the edges. ${ }^{[5]}$ This higher stability facilitates the transfer to solid substrates as well. Additionally, free surfactant molecules can adsorb to the particle surfaces and introduce further negative charges. As will be shown below, this leads to an increase in electrostatic repulsion that counteracts van der Waals and capillary attractions and induces a higher degree of order in the monolayer. Finally, the presence of a surfactant lowers the surface tension of the subphase. As the capillary forces acting on the particles is proportional to the surface- or interface tension, the addition of surfactants significantly lowers the magnitude of capillary attraction. ${ }^{[202]}$ The reduced attraction increases the time that is available for the colloids to find their minimum free energy position and thus, further increases the order of the monolayer. 
The SDS concentration was varied between 0.1 - and $1 \mathrm{mmol} \cdot \mathrm{l}^{-1}$. Characteristic electron micrographs of monolayers crystallized with these concentrations are shown in Figure 5.2.2. Highest order was achieved using the lowest SDS concentration $\left(0.1 \mathrm{mmol} \cdot \mathrm{l}^{-1}\right)$ as can be seen in b). An excellent short range order is visible. The long range order is further highlighted in a lower magnification image (e) that shows single crystalline domains with dimensions of several hundreds of square micrometers. An increase in SDS concentration up to $0.5 \mathrm{mmol} \cdot \mathrm{l}^{-1} \mathrm{still}$ produced monolayers with a high degree of order. Nevertheless, individual particles were found on top of the monolayer, thus indicating an increasing stiffness of the surfactant layer that started to interfere with the crystallization process (c). At a SDS concentration of $1 \mathrm{mmol}^{-1} \mathrm{l}^{-1}$, no complete colloidal monolayer is formed. Instead, small patches of colloidal monolayer and free surfactant were present at the surface (d). The amount of SDS is high enough to cover extensive parts of the surface and impedes the crystallization process.
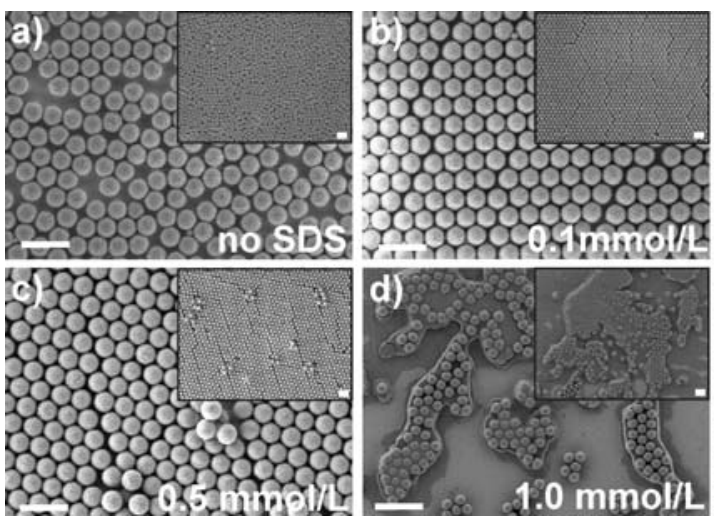

d)

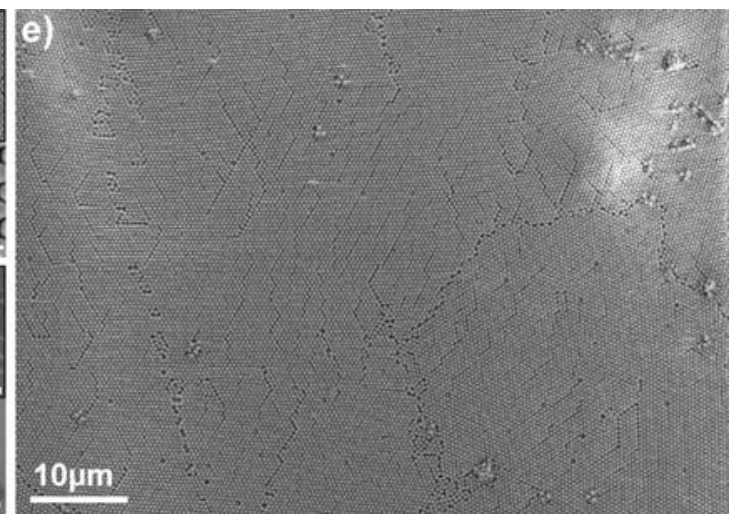

Figure 5.2.2. Effect of SDS addition to the water subphase on the quality of the resulting monolayers: a-d) Systematic variation of the SDS concentration and resulting monolayers; e) low-magnification image highlighting the high degree of order of a monolayer produced with $0.1 \mathrm{mmol} \cdot \mathrm{l}^{-1} \mathrm{SDS}$ in the water subphase.

Furthermore, the $\mathrm{pH}$ value of the water subphase was used to tune the assembly process. The particles featured carboxylic acid groups at their surface. Increasing the $\mathrm{pH}$ value induces a higher charge density at the particle surfaces as the acidic groups are more and more deprotonated (see chapter 5.2.3.2.1. for experimental verification ${ }^{[73]}$ ). This induces an increase in electrostatic repulsion that counteracts attractive van der Waals and capillary forces and allows a higher ordering of the monolayer. ${ }^{[38,88]}$ A higher crystalline order in the monolayer results as the particles remain mobile for a longer time which is a requirement for the ordering process. At lower $\mathrm{pH}$ values (i.e. screened electrostatic repulsion), colloids brought into close vicinity by capillary forces immediately feel the attractive van der Waals forces that drives them into close contact. By increasing the electrostatic repulsion, the energy barrier for a close contact is increased and the individual particles have sufficient time and mobility to find the 
energetic minimum and crystallize in their minimum free energy position as a hexagonally ordered lattice. Figure 5.2.3 shows examples of a monolayer of polystyrene particles with a diameter of $422 \mathrm{~nm}$ at $\mathrm{pH}$ values of 6 (a) and 11 (b). The higher order of the latter is clearly observable.
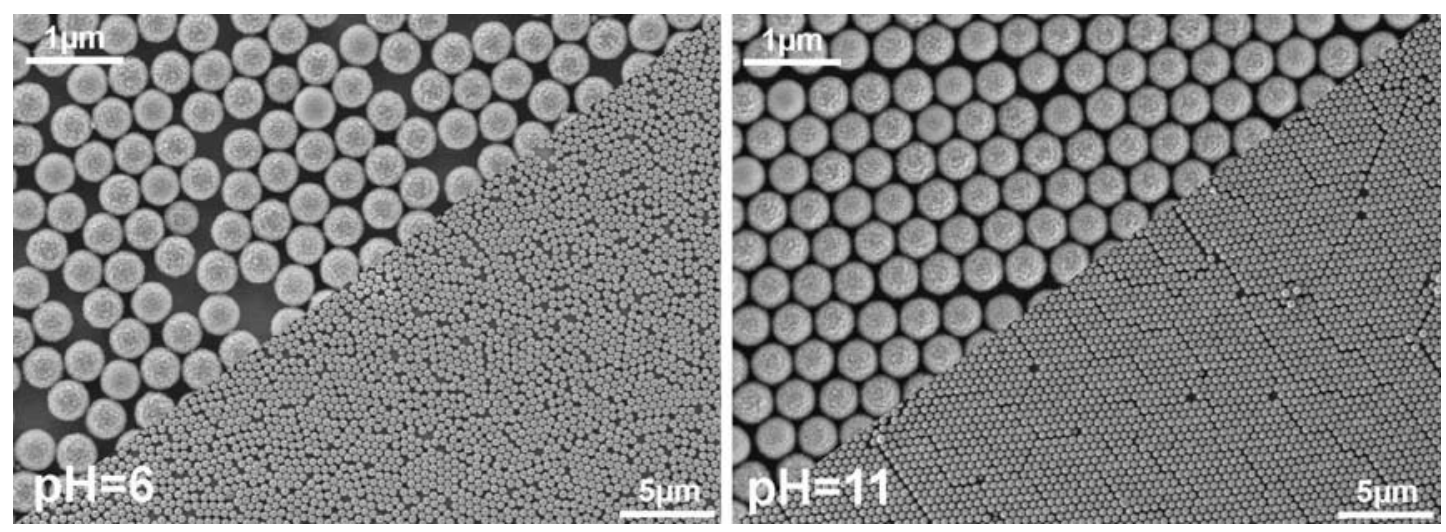

Figure 5.2.3. Effect of the $\mathrm{pH}$ value of the subphase on the quality of the resulting monolayer for carboxylic acid functionalized particles.

\subsubsection{Conclusion}

In brief, an extremely simple and reliable technique for the preparation of close-packed monolayers was developed. Dispersions of colloids in ethanol water mixtures were added to the air/water interface using a tilted glass slide. Close-packed monolayer patches formed immediately and grew in size upon further addition. The quality of the resulting twodimensional crystal could be significantly improved by addition of small amounts of SDS or adjusting the $\mathrm{pH}$ (for carboxylic acid functionalized particles). Compared to the conventional assembly technique on a Langmuir trough, the method presented provides a substantial simplification as no special apparatus is needed and time consuming compression and cleaning steps can be circumvented. 


\subsubsection{Non-close-packed monolayers by plasma induced size reduction}

\subsubsection{Introduction}

The preparation of close-packed monolayers has matured into a widely used technique and finds applications for the fabrication of nano-structures in colloidal lithography. However, the classical method is only able to create triangular-shaped structures that reflect the shape of the interstitial sites of the monolayer. ${ }^{[4-5]}$ Using individual colloids, the construction of more complex structures (e.g. rings, ${ }^{[128]}$ discs, ${ }^{[127]}$ crescents $^{[129]}$ ) has been realized by using more sophisticated evaporation and etching protocols.

In order to create these kinds of structures in symmetric arrays, the controlled preparation of non-close-packed monolayers is a key step. As discussed in chapter 3.3.2, several approaches have recently been demonstrated. Isa et al. prepared non-close-packed arrays of colloidal particles by assembly at the oil-water interface and demonstrated high control over interparticle spacing. ${ }^{[74]}$ Similarly, assembly at the air/water interface has been exploited for the preparation of non-close-packed monolayers. ${ }^{[203-204]}$ An alternative approach consists of the preparation of close-packed monolayers that are exposed to plasma in order to yield non-close-packed arrangements. ${ }^{[95,136,205]}$ Several researchers have applied this process. However, most did not focus on the etching process but rather on the construction of nanostructures themselves. ${ }^{[10,136]}$ Recently, the process has been carefully examined by Plettl and co-workers. ${ }^{[95]}$ Using sophisticated equipment and temperatures of $-150{ }^{\circ} \mathrm{C}$, they demonstrated an isotropic etching process that led to ordered non-close-packed architectures with particles that retained their spherical shape and lattice position.

In this study, a commercially available, standard plasma cleaning apparatus (model femto, diener electronics, Nagold, Germany) as being widely available in (surface-)chemistry laboratories was used to investigate the etching process and to determine etching rates and the limitations of the process. Beside an accurate investigation on the etching conditions, the shape and lattice positions of the particles at different stages of the etching process were investigated by electron microscopy. This is of special interest for colloidal lithographic applications that require round shapes and space available underneath the colloids for the creation of complex nanostructures. 


\subsubsection{Results and Discussion}

In general, the diameter of the particles decreased linearly with increasing etching time until reaching a saturation regime upon which no further decrease in size was detected. As will be examined in detail below, the saturation regime represents the threshold value for a controlled size reduction process: in the linear regime, the particles retain a roughly spherical shape that is increasingly transformed into a lentil-like shape for longer etching times (a detailed electron microscopy study is presented below). At the saturation diameter, the particles lose their spherical character and collapse to the surface forming an ill-defined residue. A characteristic image of a monolayer in the collapsed, destroyed state is shown in Figure 5.2.5. The linear dependency of the decrease in particle diameter with etching time has also been found by Plettl ${ }^{[95]}$ and Blättler. ${ }^{[206]}$ With an etching rate that is assumed to be constant in time, a shell of the thickness $\mathrm{d} r$ is etched off the particle by the plasma in the time $\mathrm{d} t$. Thus, the radius of the particle will decrease linearly. The model is valid as long as the shape of the colloid is still reasonable spherical and the surface remains homogeneous. In the course of the etching process, deviations from the linear relationship are expected due to the deformation of the colloids into a more lentil like shape and the increase in roughness of the particle surface. The rates determined for all different conditions are summarized in Table 5.2.1. The etching experiments were partially performed by Sebastian Goerres you worked as a summer student under my supervision.

\section{Effect of Plasma Power}

In a first set of experiments, the effect of plasma power on the colloidal particles was investigated. As plasma gas, oxygen was used with a flow rate of $3 \mathrm{sccm}$ (standard cubic centimeters per minute). The substrate temperature was fixed to $20^{\circ} \mathrm{C}$ using a water/ethylene glycol unit to cool the sample tray.

Figure 5.2.4 shows the resulting diameter vs. etching time diagrams for polystyrene colloids with a diameter of $408 \mathrm{~nm}$ (a) and $246 \mathrm{~nm}$ (b). As expected, a higher plasma power induces a faster shrinkage of the colloids. The etching rates for both different colloid sizes close resemble each other $\left(44 \mathrm{~nm} \cdot \mathrm{min}^{-1}\right.$ and $23 \mathrm{~nm} \cdot \mathrm{min}^{-1}$ for the $408 \mathrm{~nm}$ colloids at high/low power; $37 \mathrm{~nm} \cdot \mathrm{min}^{-1}$ and $22 \mathrm{~nm} \cdot \mathrm{min}^{-1}$ for the $246 \mathrm{~nm}$ colloids at high/low power, respectively), indicating that the size reduction is not dependent on the size of the colloids as long as both are composed of identical polymers. Using high plasma power, the saturation regime that indicates the break-down of the controlled size reduction is reached within minutes. A typical example of a monolayer of $408 \mathrm{~nm}$ colloids in the collapsed state is shown in Figure 5.2.5. Hence, in order 
to gain a better control on the etching process, $15 \mathrm{~W}$ plasma power was applied for all following experiments. The value represents the threshold energy that needs to be applied to reach stable plasma conditions in the set-up used, being necessary for a controlled size reduction.

Next, the effect of different oxygen flow rates on the etching process was investigated. Figure 5.2.4c and $\mathrm{d}$ shows the diameter vs. etching time diagrams for the same colloids subjected to oxygen plasma with a power setting of $15 \mathrm{~W}$ and flow rates of $3 \mathrm{sccm}$ and $10 \mathrm{sccm}$, respectively. The lower flow rates induce a faster etching process with etching rates of $23 \mathrm{~nm} \cdot \mathrm{min}^{-1}$ (408 nm colloids) and $22 \mathrm{~nm} \cdot \mathrm{min}^{-1}$ (246 nm colloids) in agreement with investigations on plasma processes from literature. ${ }^{[207-208]} 10 \mathrm{sccm}$ oxygen flow significantly reduced the etching rates to $16 \mathrm{~nm} \cdot \mathrm{min}^{-1}$ ( $408 \mathrm{~nm}$ colloids) and $18 \mathrm{~nm} \cdot \mathrm{min}^{-1}$ ( $246 \mathrm{~nm}$ colloids). The diagrams also show the linear dependency of the colloidal diameter with etching time. Except for small particles with low flow rates (Figure 5.2.4b), the saturation regime is not reached after the maximum etching time of $10 \mathrm{~min}$. This is highly desired as it allows very precise control the etching process in order to adjust both colloidal diameters and interparticle distances in the monolayer.
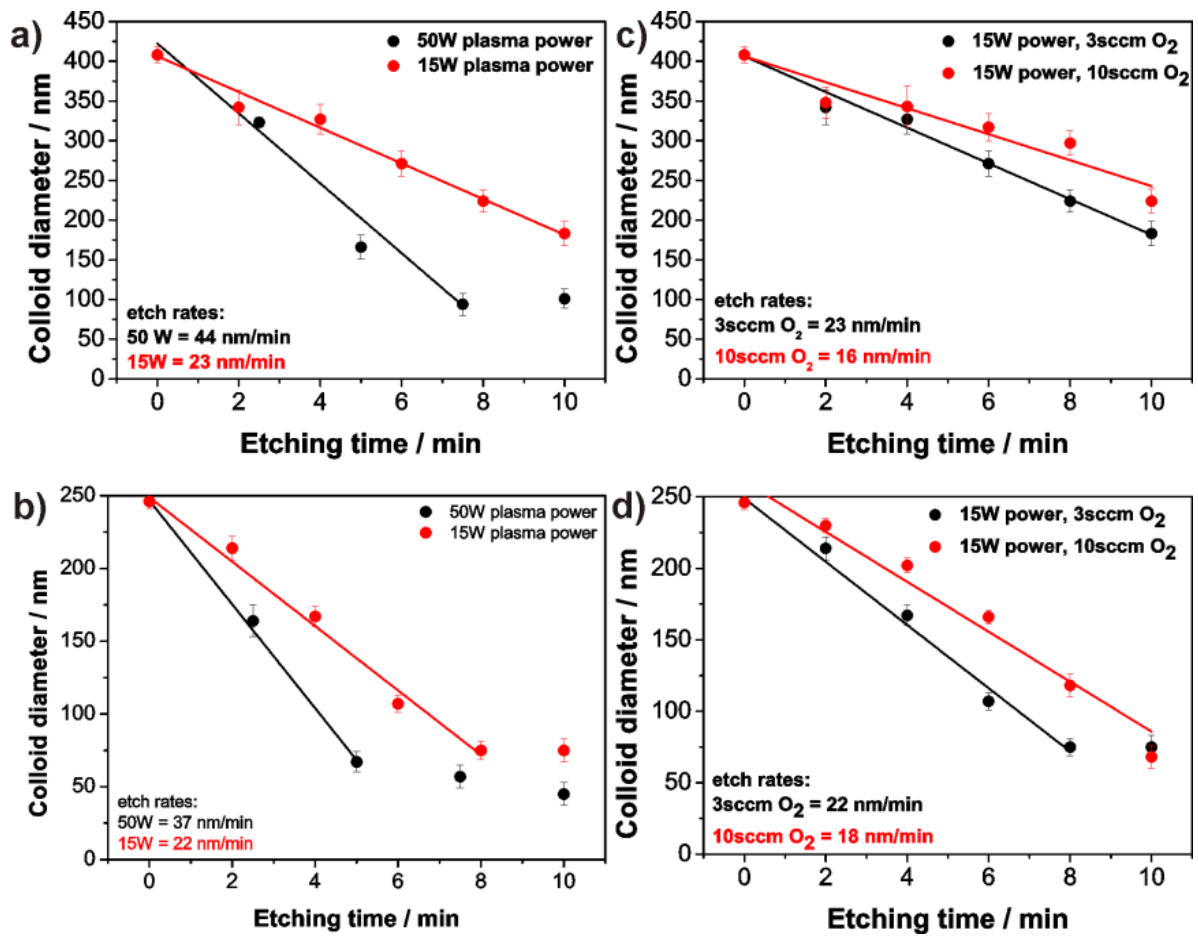

Figure 5.2.4. Effect of plasma power and gas flow rates on the size reduction of polystyrene monolayers. a,b) Effect of plasma power $(50 \mathrm{~W}$ vs. $15 \mathrm{~W})$ on the size reduction of monolayers with an initial size of (a) $408 \mathrm{~nm}$ and (b) $246 \mathrm{~nm}$. c,d) Effect of different oxygen gas flow rates ( $3 \mathrm{sccm}$ and $10 \mathrm{sccm}$ ) on the etching behavior; c) initial size $408 \mathrm{~nm}$; d) initial size $246 \mathrm{~nm}$. The etching rates are given as insets in the diagrams. 

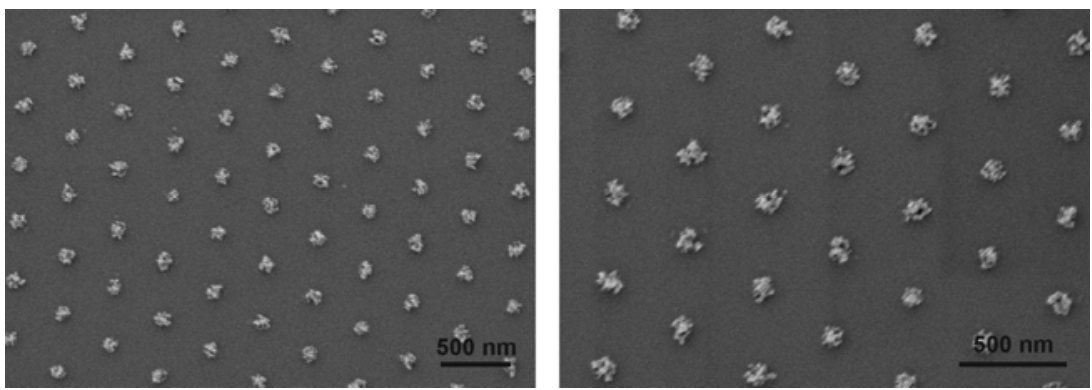

Figure 5.2.5. Monolayer of $408 \mathrm{~nm}$ colloids in the collapsed state. The individual colloids lost their spherical shape and collapsed onto the surface. The monolayer was produced by applying oxygen plasma with $3 \mathrm{sccm}$ gas flow and $50 \mathrm{~W}$ plasma power for $10 \mathrm{~min}$.

\section{Effect of polymer material of the colloids}

To test the behavior of different polymeric materials during the etching process, poly(methyl methacrylate) colloids with a diameter of $239 \mathrm{~nm}$ and polystyrene particles with a similar diameter $(246 \mathrm{~nm})$ were subjected to identical plasma conditions. Figure 5.2.6 compares the etching rates of the two types of particles for $15 \mathrm{~W}$ plasma power and oxygen plasma flow rates of 3- and $10 \mathrm{sccm}$. The etching is much faster with the PMMA particles and rates of $71 \mathrm{~nm} \cdot \mathrm{min}^{-1}\left(3 \mathrm{sccm} \mathrm{O} \mathrm{O}_{2}\right)$ and $50 \mathrm{~nm} \cdot \mathrm{min}^{-1}\left(10 \mathrm{sccm} \mathrm{O}_{2}\right)$ were determined. The finding is in good agreement with reports on polymer- and block copolymer thin film etching processes reported in the literature. ${ }^{[209-211]}$ The slower etching rates of polystyrene are attributed to the higher chemical stability of the aromatic ring. It is concluded that PMMA is not the material of choice for a controlled fabrication of non-close-packed monolayers as the rates are too high to allow for a precise adjustment of the colloidal diameter. It should be noted that an increase in etching rates can be readily achieved by increasing the plasma power. Hence, if short etching times were required, e.g. for industrial applications, the combination of polystyrene particles with $50 \mathrm{~W}$ plasma power allows a more precise adjustment of diameters and distances in the monolayer as compared to PMMA.
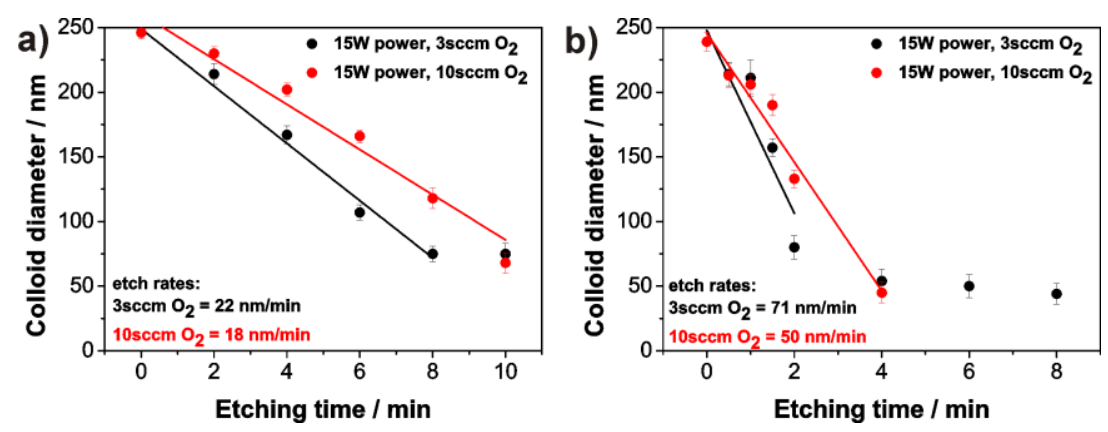

Figure 5.2.6. Effect of polymer composition for two different oxygen flow rates for $246 \mathrm{~nm}$ polystyrene (a) and $239 \mathrm{~nm}$ poly(methyl methacrylate) colloidal monolayers (b). 


\section{Electron microscopy investigations on the shape of the}

\section{colloids during the etching process}

Three characteristics of the colloids subjected to the etching process are of key importance for any lithographic application. First, the diameters need to be precisely adjusted in order to tailor nanostructure arrays with defined sizes. Second, the symmetry of monolayer should not be lost during the etching process in order to produce metal nanostructure arrays with a high ordering. Therefore, during the etching process, the colloidal particles need to retain their individual positions and shrink without lateral movement. Third, the particles need to keep their spherical shape in order to produce architectures with the maximum flexibility with regards to colloidal lithography. This becomes obvious when realizing that many processes for complex structure construction take advantage of the substrate regions shaded by the colloids. ${ }^{[10,129,212]}$ Once the particles completely lose their shapes, they can still be used for the construction of e.g. disc-like structures ${ }^{[213]}$ or as etching masks for substrate patterning, ${ }^{[121,214]}$ but any more complicated geometries (i.e. evaporation and etching under varying angles to exploit the area shaded by the colloid) cannot be produced by a lithographical process anymore. In this chapter, scanning electron microscopy is used to image the monolayers at different stages of the etching process in order to access possibilities and limitations of the etching process.
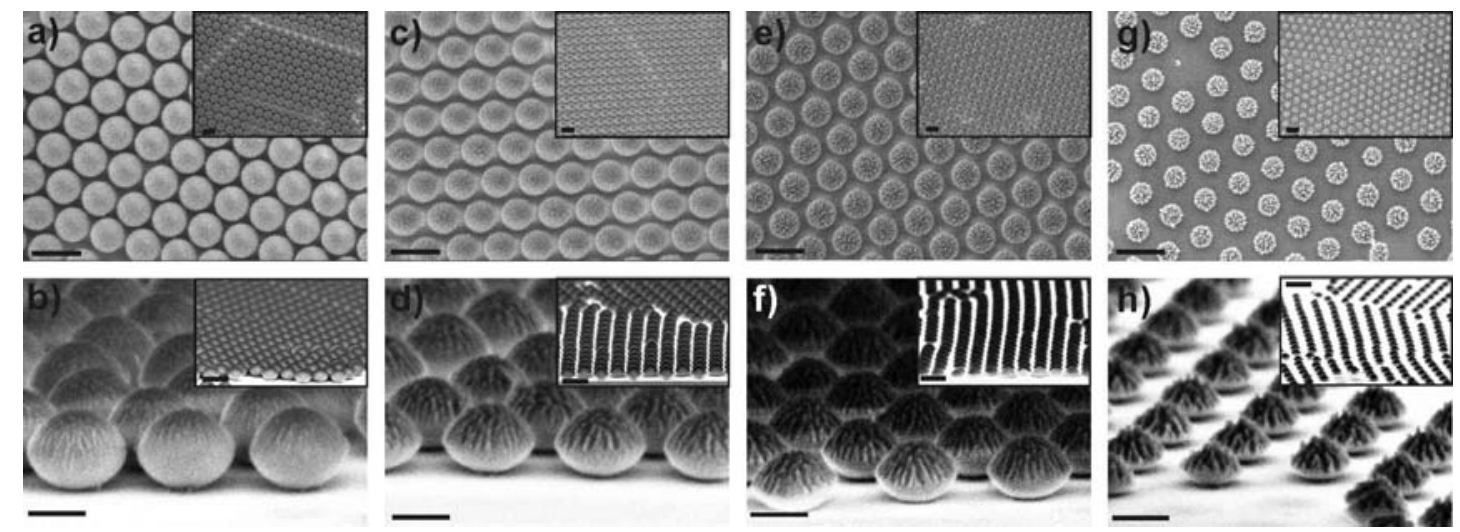

Figure 5.2.7 Examination of shape and position of the individual colloids of a poly-styrene monolayer subjected to oxygen plasma $(15 \mathrm{~W}, 10 \mathrm{sccm})$. The size of the original colloids was $408 \mathrm{~nm}$. The upper row shows top view images, the lower row side view images. The insets show lower magnifications of the same sample. The scale bars are: upper row: $500 \mathrm{~nm}$; insets: $1 \mu \mathrm{m}$; lower row: $200 \mathrm{~nm}$; insets: $500 \mathrm{~nm}$.

Plasma was applied for the following times: a, b) 4 min; c,d) 6 min; e,f) 8 min; g,h) $10 \mathrm{~min}$.

Figure 5.2.7 presents electron microscopy images of a colloidal monolayer comprised of $408 \mathrm{~nm}$ polystyrene latex particles in the course of the plasma etching process. In the top-view images (Figure 5.2.7, upper row), the high symmetry of the monolayer at all etching stages is well visible. Hence, it can be concluded that plasma etching does not induce any lateral disorder to the non-close-packed monolayer. The side-view images (Figure 5.2.7, lower row) reveal the 
shape of the individual particles. It can be seen that the particles do not shrink isotropically. Instead, the etching is more efficient on the top of the particles, resulting in lentil-shaped colloids after several minutes of etching. Increasing etching times roughens the particle surfaces of the top parts. After long etching times $(10 \mathrm{~min})$, the deviation from a spherical shape is pronounced. However, keeping in mind applications in colloidal lithography it should be noted that even for the longest etching time investigated $(10 \mathrm{~min})$, the particles still featured a round shape at their lower hemisphere and were not completely collapsed. Thus, it is still possible to use them as evaporation masks because they continue to shade a certain amount of substrate space (h). At that stage, the particle diameter is reduced to approximately half its initial value. Further etching induced a collapse of the already strongly deformed particles into irregular shaped structures as previously shown (Figure 5.2.5).
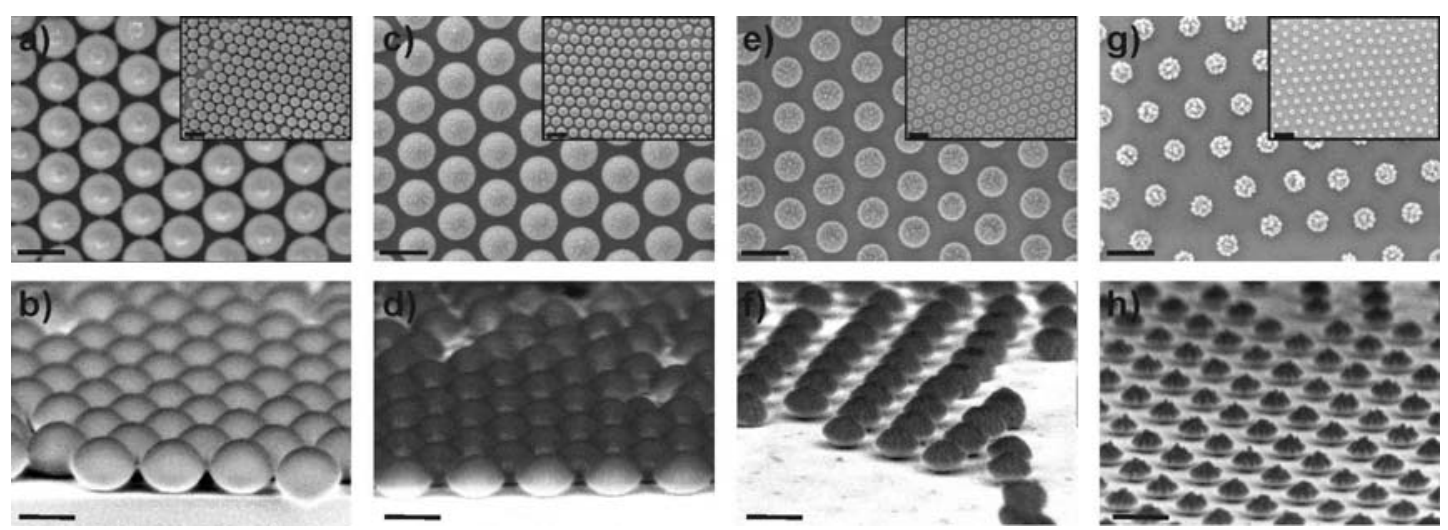

Figure 5.2.8. Examination of shape and position of the individual colloids of a polystyrene monolayer subjected to oxygen plasma ( $15 \mathrm{~W}, 10 \mathrm{sccm})$ with an initial size of $246 \mathrm{~nm}$. The upper row shows top view images, the lower row side view images. The insets show lower magnifications of the same sample. The scale bars are: upper row: $200 \mathrm{~nm}$; insets: $500 \mathrm{~nm}$; lower row: $200 \mathrm{~nm}$. Plasma was applied for the following times: a, b) $2 \mathrm{~min}$; c,d) 4 min; e,f) 6 min; g,h) 8 min.

Figure 5.2.8 shows the electron microscopy images of monolayers made of polystyrene particles with a diameter of $246 \mathrm{~nm}$ in the course of the etching process. In agreement with all observations on the larger colloids, it can again be stated that the lateral symmetry is retained during all stages of the etching process. The shape of the individual particles is gradually transformed into a more flattened, ellipsoidal structure; accompanied by an increase in roughness for longer etching times. However, until the particle diameter is reduced to less than half its initial value $(8 \mathrm{~min})$, the shape of the particles is still suitable for any lithographical applications.

It can thus be estimated that the etching procedure described here produces non-close-packed monolayer with the same, high symmetry as the parental, close-packed monolayers. For polystyrene as colloid material, the size can be reduced to roughly half the initial diameter 
before deviations from spherical shape completely impede the use in sophisticated lithographic applications. The relation seems to be independent of the initial colloid diameter.

Finally, the PMMA colloids used in Figure 5.2.6b were investigated by electron microscopy as well. Melting of the colloids in the electron beam inhibited the recording of side-view images. Please note that the etching times applied for the individual stages of the process are substantially shorter compared to the ones for the polystyrene monolayers shown above. The top view images shown in Figure 5.2.9 highlight the symmetry of the monolayers that is retained in a way similar to polystyrene colloids. The individual spheres, however, seem to be more roughened compared to the polystyrene particles shown above. We attribute this behavior to the decreased resistance of PMMA towards the plasma that leads to particles being more severely attacked by the oxygen species generated in the plasma. The last picture in the row shows a particle array in the saturation regime. The spherical shape is lost and the particles are flattened and irregularly shaped. Once this stage is reached, further plasma treatment does not induce any changes in shape or diameter of the colloids.
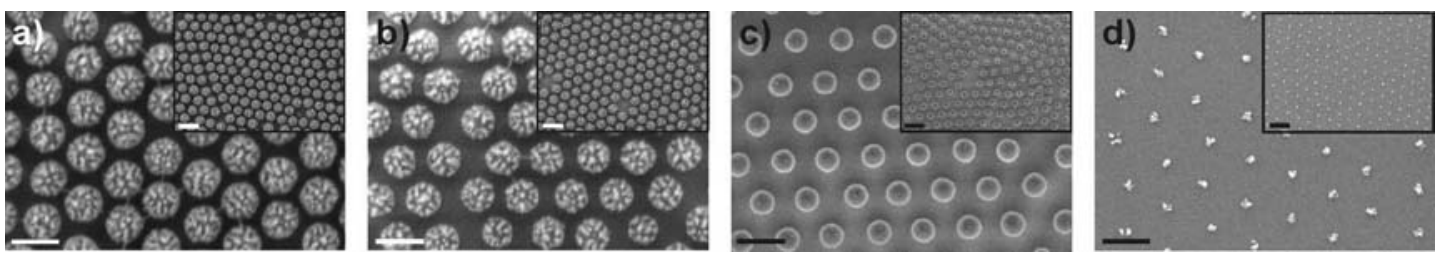

Figure 5.2.9. Examination of shape and position of the individual colloids of a poly(methyl methacrylate) monolayer with initial diameter of $239 \mathrm{~nm}$ subjected to oxygen plasma $(15 \mathrm{~W}, 10 \mathrm{sccm})$. The insets show lower magnifications of the same sample. The scale bars are: $200 \mathrm{~nm}$; insets: $500 \mathrm{~nm}$. Plasma was applied for the following times: a) $60 \mathrm{~s}$; b) $90 \mathrm{~s}$; c) $120 \mathrm{~s}$; d) $240 \mathrm{~s}$.

\section{Effect of plasma composition}

The effect of the plasma gas was investigated as well. Figure 5.2.10 summarizes all etching experiments performed with different gas compositions. All colloids in the study were subjected to oxygen and argon plasma with similar flow rates $(3 \mathrm{sccm})$. As can be seen from Figure 5.2.10a to c, oxygen plasma leads to a more rapid shrinking of the particles compared to argon. For polystyrene particles, the etching rates were $22 \mathrm{~nm} \cdot \mathrm{min}^{-1}$ (246 nm colloids) and $23 \mathrm{~nm} \cdot \min ^{-1}$ (408 $\mathrm{nm}$ colloids) for oxygen gas and $10 \mathrm{~nm} \cdot \mathrm{min}^{-1}(246 \mathrm{~nm})$ and $11 \mathrm{~nm} \cdot \mathrm{min}^{-1}$ $(408 \mathrm{~nm})$ for argon respectively. For PMMA as colloid material, oxygen plasma induced a decrease in diameter of $71 \mathrm{~nm} \cdot \mathrm{min}^{-1}$; application of argon lead to rate of $34 \mathrm{~nm} \cdot \mathrm{min}^{-1}$. For all samples investigated, the etching rate of oxygen is approximately two times higher than the one of Argon. Hence, another means of controlling the etching rate and by this, the final colloidal diameter is at hand. It should be mentioned that argon plasma produced particles with a rougher colloid surface compared to oxygen plasma treated particles with a similar diameter. 
Figure 5.2.10d shows a more detailed examination of plasma compositions with $408 \mathrm{~nm}$ colloids. The composition was systematically varied from pure oxygen $(3 \mathrm{sccm}$; same data as Figure 5.2.10a) via ratios of 2:1 oxygen:argon, 1:1, 1:2 to pure argon (equaling the second graph of Figure 5.2.10a). The etching rates of all oxygen containing plasmata did not differ significantly and the non-close-packed monolayers produced by the different mixtures resembled each other. Only pure argon led to a drastic reduction of the shrinking process. It can be concluded that the presence even of small amounts of oxygen molecules in the plasma strongly accelerates the etching process. Oxygen molecules are the more efficient species in the decomposition process of the polymeric material as chemical decomposition by insertion of peroxo-radicals into the backbone followed by chain scission is the dominating process of the plasma etching. ${ }^{[210]}$ In contrast, argon species generated in the plasma are chemically inert and physical decomposition by surface bombardment of ions is the dominating mechanism. This also explains the higher roughness of colloids etched with argon as they are subjected to higher impact energies by the heavier argon ions. Surface functionalization, related to chemical etching, has been reported to only occur due to water impurities in the argon plasma or by environmental oxygen after the plasma treatment. ${ }^{[11,215]}$
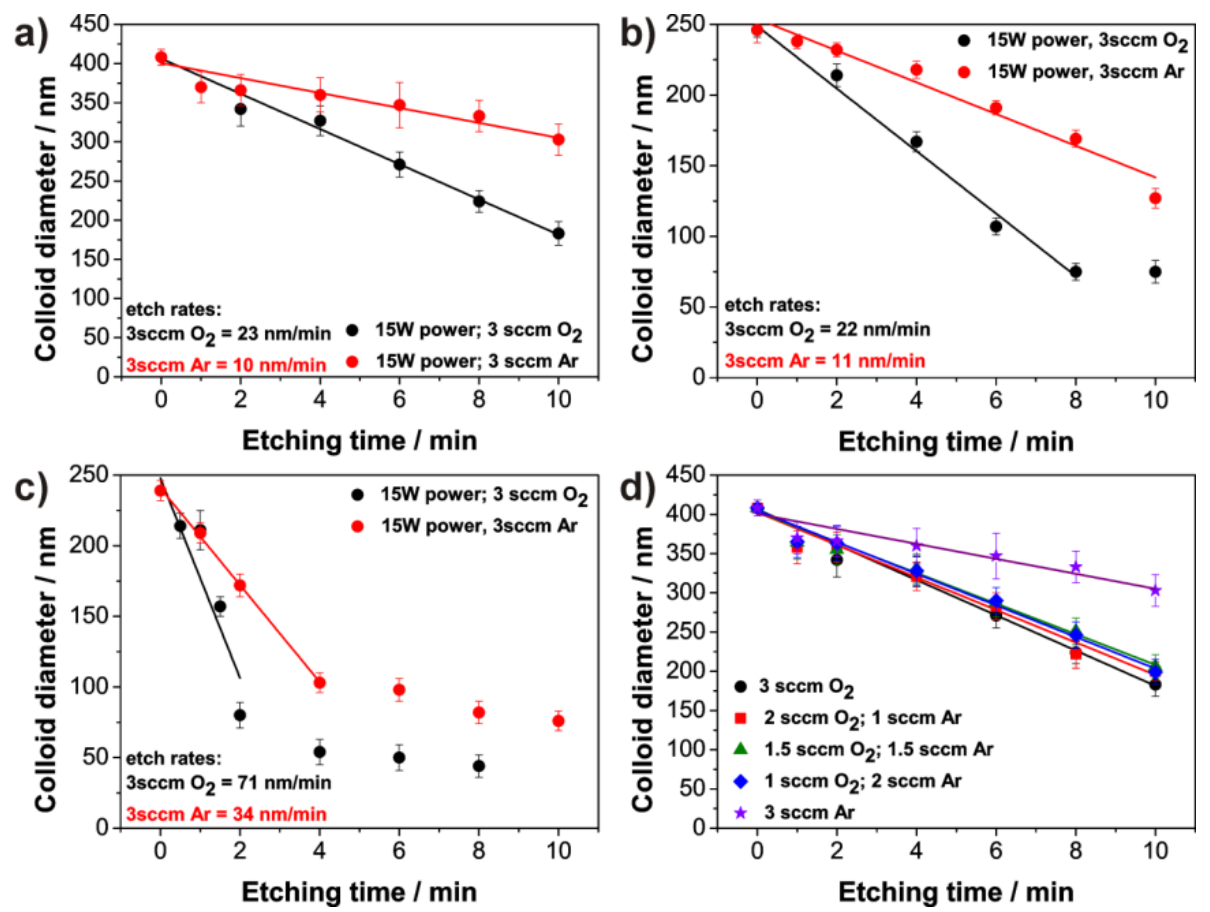

Figure 5.2.10. Effect of plasma composition on the etching behavior: a-c) Comparison of pure oxygen to pure argon plasma for $408 \mathrm{~nm}$ PS colloids (a); $246 \mathrm{~nm}$ PS colloids (b) and $239 \mathrm{~nm}$ PMMA colloids; (c),

(d) more detailed investigation on different oxygen:argon ratios for $408 \mathrm{~nm}$ PS colloids. 


\section{Effect of substrate temperature on the etching process}

The substrate temperature was varied in order to examine possible changes in the etching process. Using a water/ethylene glycole mixture in a cryostat connected to the bottom of the plasma chamber, the substrate temperature was controlled between $20^{\circ} \mathrm{C}$ and $0{ }^{\circ} \mathrm{C}$. Figure 5.2.11 shows the resulting diameter vs. etching time diagrams. Decreasing the substrate temperature led to a slight decrease in etching rates, both for oxygen and argon plasma applied. The effect was not very pronounced and limited to few nanometers per minute. Concluding this set of experiments it should be stated that the substrate temperature does not have a significant effect on the etching rates and particle morphologies for the temperature range investigated. However, controlling the temperature of the plasma chamber is important as uncontrolled temperature led to strong increases of the temperature ${ }^{[210]}$ of the substrate in the course of the etching process with the drastic consequences on the resulting particle arrays. First, the etching rates were not reproducible and featured strong deviations from the typical linear dependencies of the controlled process. Furthermore, etching times of $5 \mathrm{~min}$ and longer resulted in particles with a completely destroyed shape; somewhat resembling popcorn. Hence, even though the precise adjustment of temperature does not seem to be very significant for the process, cooling of the plasma chamber is an important requisite for successful size reduction processes.
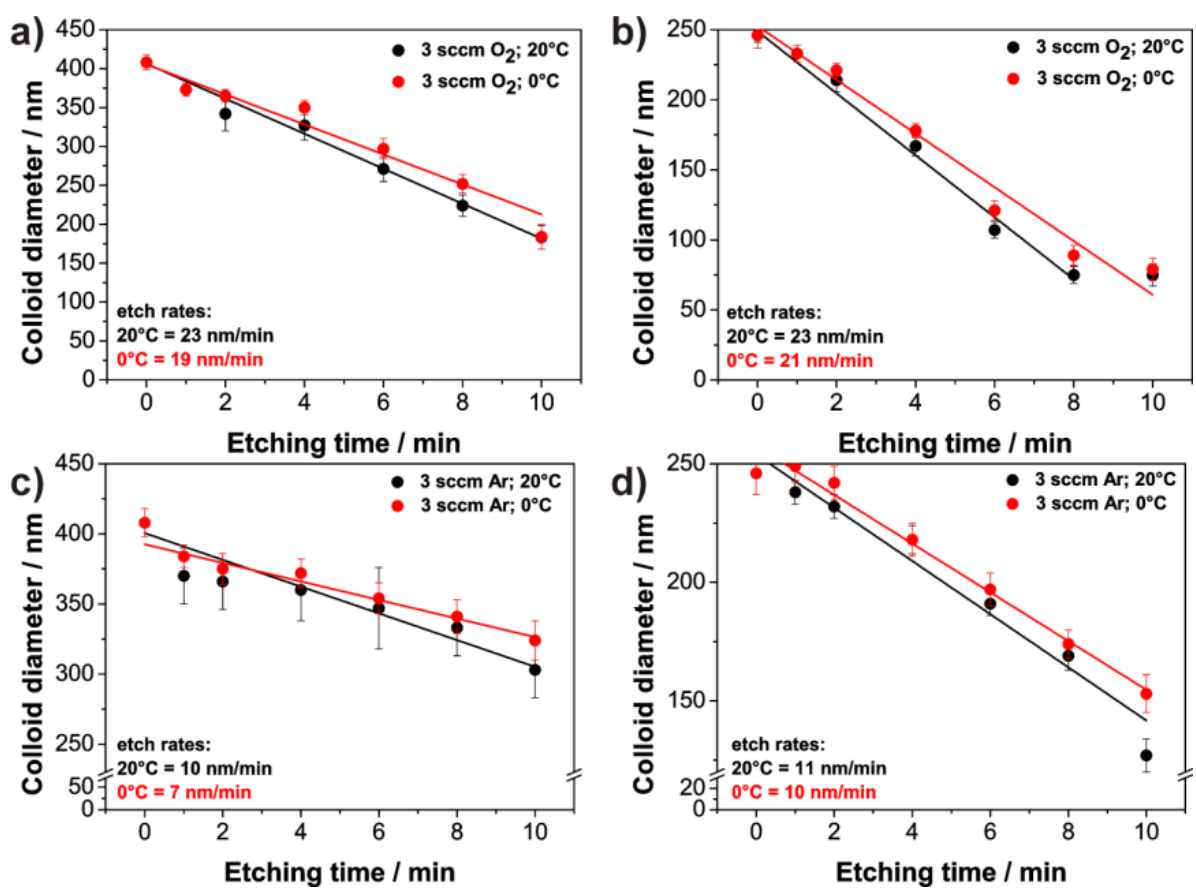

Figure 5.2.11. Effect of substrate temperatures on etching rates for $408 \mathrm{~nm}$ PS colloids (left row) and $246 \mathrm{~nm}$ colloids (right row) using oxygen plasma (upper row) and argon plasma (lower row), respectively. 


\section{Effect of the substrate}

Finally, the effect of the substrate on the plasma induced size reduction process was accessed experimentally. Colloidal monolayers were deposited on substrates typically applied in colloidal lithography (Si-wafer and glass) and treated with oxygen plasma with a flow rate of $10 \mathrm{sccm}$. As glass substrate, conventional microscopy slides were chosen. Both the microscopy slide as well as the silicon wafer were also covered with a thin gold film $(50 \mathrm{~nm})$, as typically applied in lithographic processes. Furthermore, the silicon wafer was covered with a $\sim 10 \mu \mathrm{m}$ thick film of photoresist (SU-8) to investigate effects of a thicker, insulating layer on the silicon wafer. Figure 5.2.12 shows the resulting diameter-vs.-etching time diagrams. The etching rates on silicon was twice as high as compared to glass as substrate material $\left(16 \mathrm{~nm} \cdot \mathrm{min}^{-1}\right.$ for $\mathrm{Si}$ and $7 \mathrm{~nm} \cdot \mathrm{min}^{-1}$ for $\mathrm{SiO}_{2}$ ). The effect of thin film of gold is not pronounced and the etching rates resemble the native substrates. The thicker film of insulating photoresist significantly reduces the etching rate by approximately $30 \%$. We attribute this phenomenon to a higher density of charges on insulating surfaces. In contrast to a (semi)conductor that can remove the charges introduced by ions and electrons in the plasma, insulators accumulate charges on their surfaces. In the course of the etching process, the substrate is increasingly charged and thus shields likecharged species in the plasma that cannot contribute to the etching process, resulting in smaller etching rates. The phenomenon is related to smaller etching rates of silicon dioxide compared to silicon that are reported for reactive ion beam etching experiments. ${ }^{[207]}$
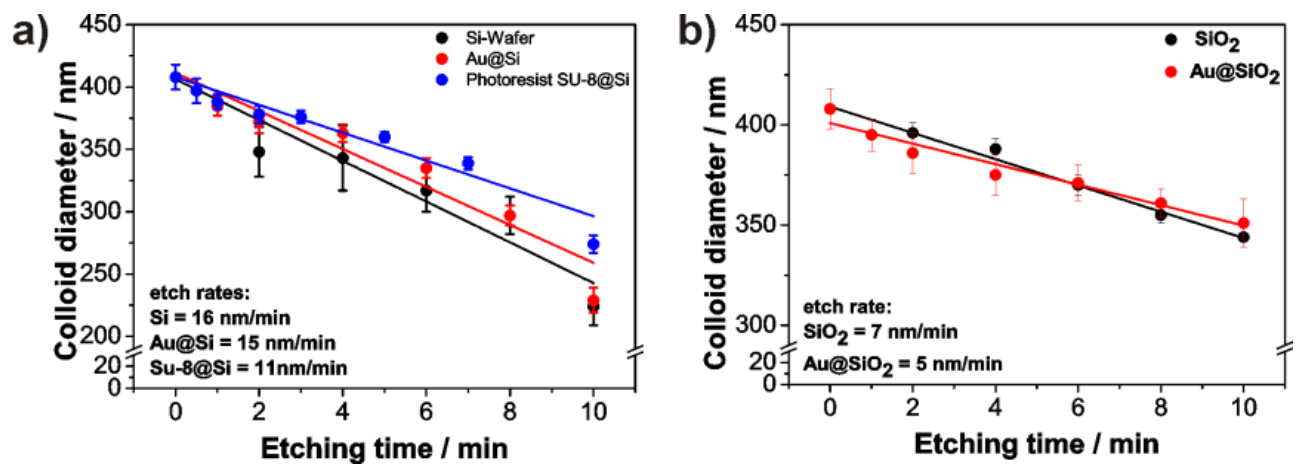

Figure 5.2.12. Effect of substrate material on the size-reduction process. a) Silicon wafer and wafer with thin films of gold and photoresist; b) microscope slide and slide with a thin gold film.

\subsubsection{Conclusions of the etching experiments}

A detailed investigation of the etching process was performed and the following conclusions can be drawn. During the etching process, no lateral movement of the colloids was observed and the non-close packed architectures exhibited the same, high lateral symmetry and order as the 
initial close-packed monolayers. In the course of the etching process, the particles' initial spherical shape deforms into lentil-like structures. The particles can be decreased up to approximately $50 \%$ of their initial diameter before completely collapsing and losing their structural integrity. Polystyrene proved to be the more suitable colloidal material compared to poly(methyl methacrylate) as the latter exhibited high etching rates and thus little control over resulting particle diameters. Oxygen plasma showed significantly higher etching rates compared to argon and the latter induced higher surface corrugation on the colloids. The substrate material drastically affected the etching rates while temperature of the substrates only marginally influenced the process. However, a control of the temperature was necessary for a successful preparation of non-close-packed monolayers to prevent high temperatures and "explosion" oingf the individual colloids. Table 5.2.1 summarizes all parameters of the etching experiments and provides the experimentally determined etching rates.

Table 5.2.1. Summary of the etching experiments.

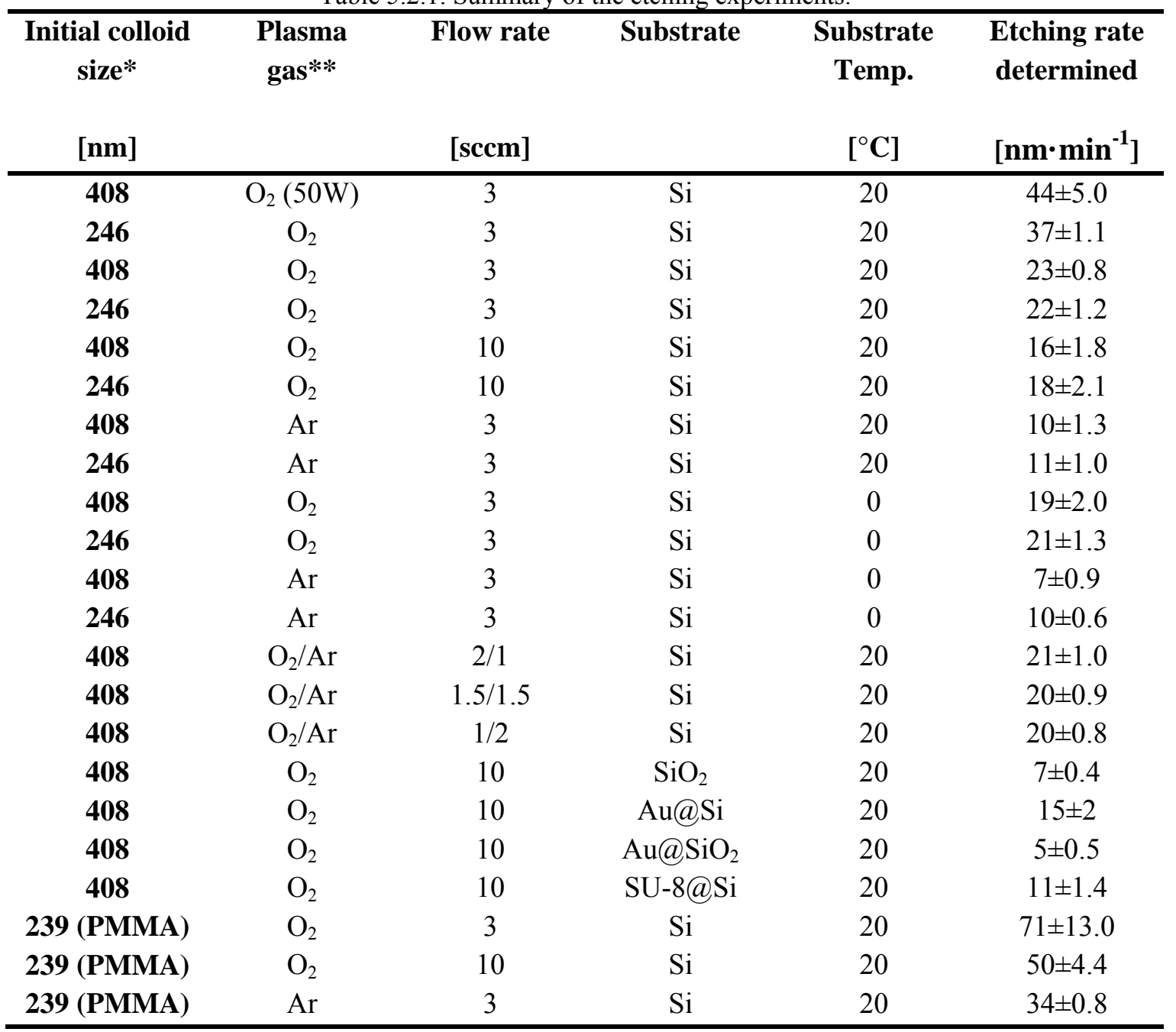

*unless otherwise stated, the polymer material was polystyrene.

**the plasma power was $15 \mathrm{~W}$ unless specified. 


\subsubsection{Color of the monolayers as a means to control the etching process}

Colloidal monolayers on solid substrates in general show strong coloration when illuminated with white light. ${ }^{[82,216-217]}$ The color strongly depends on colloidal size, lattice orientation, and interparticle spacing. One has to distinguish two different phenomena that lead to the color. First, highly crystalline monolayers with a size above approximately $500 \mathrm{~nm}$ exhibit strong iridescence in dependence of illumination and observation angles. This phenomenon, known e.g. from butterfly wings in nature, ${ }^{[13]}$ results from diffraction of light in the layered arrangement of particles in a colloidal monolayer. Light waves scattered by individual particles interfere with beams scattered from neighboring particles and give rise to amplification (constructive interference) or annihilation (destructive interference) of light with certain wavelengths. ${ }^{[82]}$ In total, brilliant intermixing of colors with strong angular dependence and a clear distinction between individual crystal domains results. Second, monolayers of sizes below $\sim 500 \mathrm{~nm}$ show continuous, uniform coloring. This color is a result of interference of light at parallel planes of the monolayer surface and the substrate. ${ }^{[216-217]}$ Crystallinity is not a requirement for this type of color as the monolayer merely acts as a thin, continuous film. The uniform coloration strongly depends on the size of the particles and can thus be used as a simple means to control the etching process. This might be of interest for example in quality control in industrial applications where electron microscopy control is time consuming and cumbersome.

Figure 5.2.13a presents UV/Vis-NIR transmission spectra of monolayers consisting of polystyrene spheres with an initial diameter of $408 \mathrm{~nm}$ at plasma etching times between 0 and $10 \mathrm{~min}$. To illustrate the strong differences in coloration of the two dimensional colloidal crystals at different etching times, photographs of 2 inch wafers covered with monolayers where taken and are presented in Figure 5.2.13b to e. The wafers were illuminated normal to the wafer surface. In the UV/Vis-NIR spectra, two clear extinction features are visible. A first, broad peak appears in the infrared regime and shifts towards smaller wavelength for longer etching times (i.e. smaller particle diameters and larger interparticle spacing). A second, more pronounced peak appears in the visible range at around $500 \mathrm{~nm}$ for the initial monolayer. A blue-shift in the course of the etching process is detected for the second maxima as well (see inset for a more detailed picture). Both shifts are indicated by arrows in the Figure. In order to explain the spectral features, a theoretical model was adopted from literature and extended to non-closepacked architectures. ${ }^{[216]}$ 

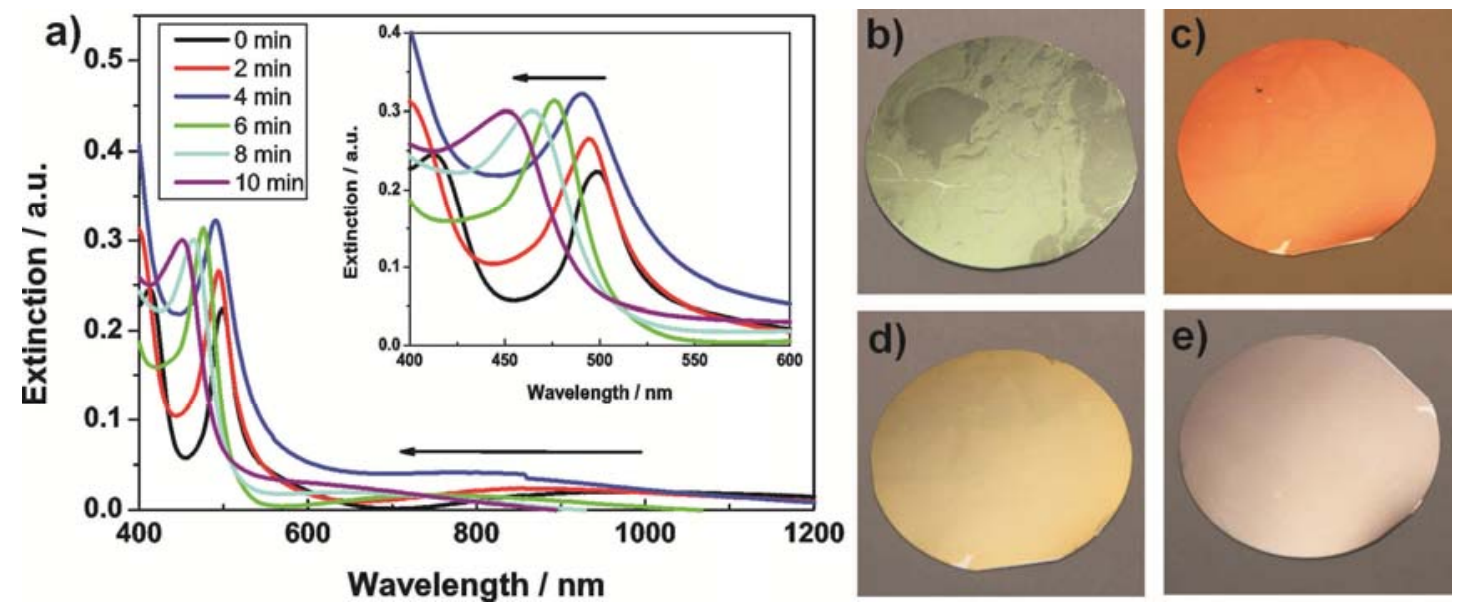

Figure 5.2.13. Optical investigation of the monolayers produced by plasma induced size reduction. a) UV/Vis-NIR spectra of colloidal monolayers reduced in size by oxygen plasma treatment for 0-10 min. The extinction maxima shift towards shorter wavelength for longer etching times (indicated by arrows); b-e) photographs of monolayers deposited on a 2 inch silicon wafer and illuminated normal to the wafer surface; b) initial close-packed monolayer; c-e) monolayer after oxygen plasma treatment for 4 min (c), $6 \mathrm{~min}(\mathrm{~d})$, and $8 \mathrm{~min}(\mathrm{e})$.

Treating the monolayer that consists of individual particles as a continuous, plane parallel film, one can apply classical optics to describe the interference between waves reflected at the surface of the thin film and substrate surface. ${ }^{[216,218]}$ The intensity of the reflected light depends on the path difference between the waves reflected at the air/colloid and the colloid/substrate interface. When illumination occurs normal to the surface, angular dependencies of the phase shift cancel out ${ }^{[216]}$ and constructive interference is obtained for a path difference of integers $(m=1,2, \ldots)$ of $\lambda$. Figure 5.2.14 shows a sketch of the geometrical situation. The monolayer is treated as a continuous thin film (light blue). Light beam 1 is reflected at the air/monolayer interface while the beam while beam 2 is reflected at the monolayer/substrate interface.

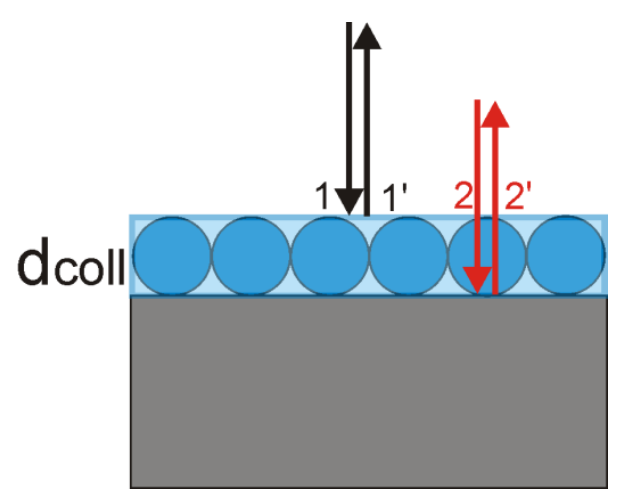

Figure 5.2.14. Interference between light beams reflected at the air/monolayer and monolayer/substrate plane. 
The additional optical path $\Delta$ that the light of beam 2 travels compared to beam 1 equals:

$$
\Delta=2 d_{\text {coll }} \cdot n_{\text {mono }}
$$

In the equation, $d_{\text {coll }}$ represents the diameter (or, more precisely, the height) of the colloids and $n_{\text {mono }}$ the effective refractive index of the monolayer. Constructive interference occurs for multiples of the wavelength $(m \lambda)$ of the incident beam. Hence, it follows that the wavelengths that are constructively reflected can be determined as:

$$
\lambda=\frac{2 d_{\text {coll }}}{m} n_{\text {mono }}
$$

Hence, reflective maxima can be calculated as:

$$
m \lambda=2 d_{\text {coll }} n_{\text {mono }}(m=1,2, \ldots)
$$

The refractive index of the monolayer $n_{\text {mono }}$ depends on the refractive index of the polymer material $n_{P S}$ (here: polystyrene), the volume fraction of the colloids $\varphi_{c o l l}$ and the refractive index of the surrounding medium, $n_{m e d}$ (here: air), and can be expressed as: ${ }^{[219]}$

$$
n_{\text {mono }}=n_{P S} \varphi_{\text {coll }}+n_{\text {med }}\left(1-\varphi_{\text {coll }}\right)
$$

Using the expression for $n_{\text {mono }}$ and taking $n_{\text {med }}=1$ for air as surrounding medium, an expression for the reflection maxima is obtained:

$$
\lambda=\frac{2}{m} d_{\text {coll }}\left[n_{P S} \varphi_{\text {coll }}+\left(1-\varphi_{\text {coll }}\right)\right]
$$

The last task for the calculation of the constructive interference peaks is the determination of the volume fraction of the colloids in the non-close-packed monolayer. Using geometric arguments, the volume fraction can be approximated as the volume fraction of a sphere with the diameter $d_{n c p}$ (being the diameter of the colloid in the non-close-packed monolayer) in a hexagonal prism with the base determined by the initial diameter of the spheres in the close-packed arrangement, $d_{0}$, and the height $d_{n c p}$ as schematically shown in Figure 5.2.15. 
a)

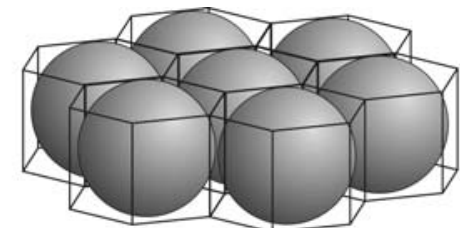

b)

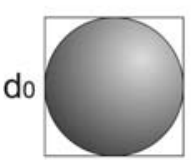

c)

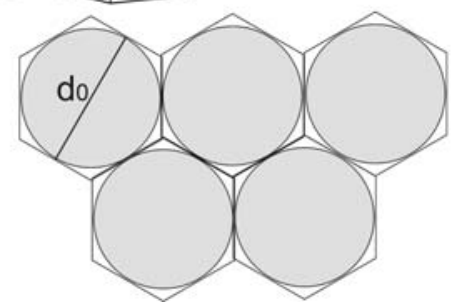

d)

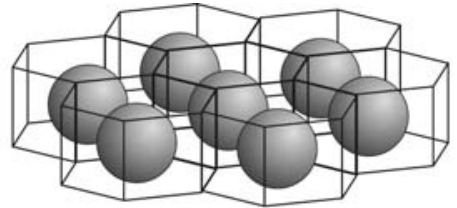

e)

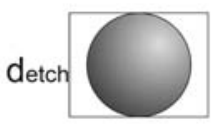

f)

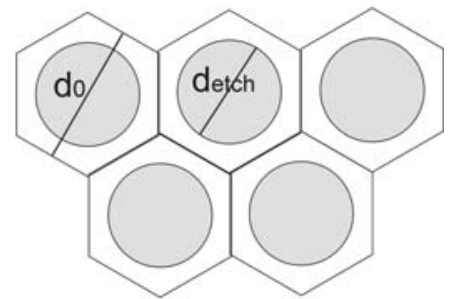

Figure 5.2.15. Model for the calculation of the volume fraction of colloids in a monolayer treated as a continuous thin film. The complete film is modeled as an array of interconnected hexagonal prisms with the base $d_{0}$. a-c) close-packed monolayer; a) 3D model of the monolayer; b) side view image: the height of the prisms equals the colloid diameter $d_{0}$; c) top view image: the base of the prism is defined by the diameter of the colloids; d-f) non-close-packed monolayer; d) 3D model of the monolayer film; e) side view: the height of the prisms equals the diameter of the etched colloids $d_{\text {etch }} ;$ f) top view image. The base of the prisms still equals the initial diameter of the colloids $d_{0}$.

The area of the prism base $A_{\text {base }}$ can be described as 6 interconnected equilateral triangles with a height of $d_{0} / 2$; the height of the prism is given by the colloid diameter $d_{\text {ncp: }}$ :

$$
V_{\text {prism }}=A_{\text {base }} \cdot d_{n c p}=\frac{3}{2 \sqrt{3}} \cdot d_{0}^{2} \cdot d_{n c p}
$$

The volume fraction $\varphi_{\text {coll }}$ can now be expressed as follows:

$$
\varphi_{\text {coll }}=\frac{V_{\text {coll }}}{V_{\text {prism }}}=\frac{\pi \sqrt{3}}{9} \cdot \frac{d_{n c p}^{2}}{d_{0}^{2}}
$$

Table 5.2.2 compares the theoretical values for the constructive interference with the experimental data extracted from Figure 5.2.13a. A refractive index for polystyrene of $n_{P S}=1.57$ was used and the wavelength dependence was neglected. ${ }^{[219]}$

A qualitative agreement between theory and experimental data is obvious. Both first $(m=1)$ and second $(m=2)$ interference maxima are reproduced and the shift towards shorter wavelengths for increasingly less densely packed architectures as found experimentally is confirmed theoretically. Quantitatively, a discrepancy between experimental data and theory is found. This can readily be explained by oversimplification of the model. The ordering of the monolayer is assumed to be perfect and defects are neglected. Thus, the volume fraction of the colloids is overestimated in the model. Additionally - and more severely the colloids are assumed to fully retain their spherical shape while in reality, their shape deforms into a lentil- 
like form. As the model uses the experimentally determined top view diameter of the colloids as the height of the thin film, the volume fraction is further overestimated as the real height is reduced due to the shape deformation. Further refinements of the model, e.g. by taking into account the deviations from spherical shape are expected to increase the quantitative agreement between model and experimental data. Considering the simplicity of the theoretical model, the qualitative agreement with experimental data was surprisingly good and all characteristic features were reproduced.

Table 5.2.2. Constructive interference maxima of a polystyrene monolayer subjected to plasma treatment.

\begin{tabular}{|c|c|c|c|c|c|}
\hline \multirow{2}{*}{$\begin{array}{c}\text { Etching time } \\
\text { [min] }\end{array}$} & \multirow{2}{*}{$\begin{array}{c}\text { Colloid } \\
\text { Diameter } \\
\text { [nm] } \\
\end{array}$} & \multicolumn{2}{|c|}{ Theoretical value } & \multicolumn{2}{|c|}{ Experiment } \\
\hline & & $\begin{array}{l}1^{\text {st }} \text { order } \\
\text { maximum } \\
\text { [nm] }\end{array}$ & $\begin{array}{c}2^{\text {nd }} \text { order } \\
\text { maximum } \\
\text { [nm] }\end{array}$ & $\begin{array}{l}1^{\text {st }} \text { order } \\
\text { maximum } \\
\text { [nm] }\end{array}$ & $\begin{array}{c}2^{\text {nd }} \text { order } \\
\text { maximum } \\
\text { [nm] }\end{array}$ \\
\hline $\mathbf{0}$ & 408 & 1097 & 549 & 1010 & 500 \\
\hline 2 & 393 & 1049 & 524 & 906 & 494 \\
\hline 4 & 388 & 1018 & 509 & 846 & 490 \\
\hline 6 & 370 & 950 & 475 & 758 & 476 \\
\hline 8 & 355 & 895 & 448 & 668 & 464 \\
\hline 10 & 344 & 834 & 428 & 650 & 450 \\
\hline
\end{tabular}

\subsubsection{Applications in colloidal lithography}

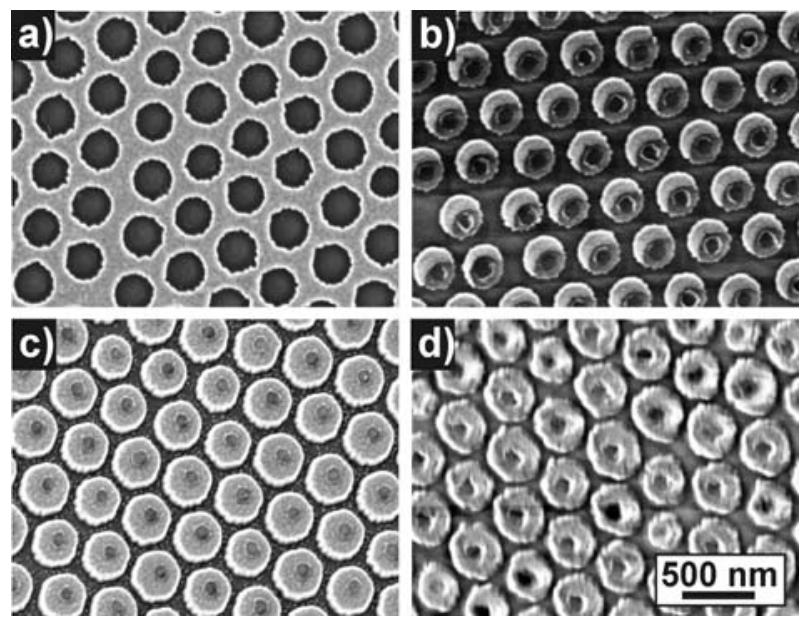

Figure 5.2.16. Application of non-close-packed monolayers in colloidal lithography: a variety of gold nanostructures can be readily created in highly ordered arrays. (a) Holes in a continuous gold film; (b) gold nanocrescents; (c) gold discs; (d) gold rings. 
In order to briefly demonstrate the versatility of the non-close-packed monolayer in colloidal lithography applications, arrays of differently shaped nanoparticles were produced. Figure 5.2.16 shows different structures that are produced with ease using non-close-packed monolayers as masks. Figure 5.2.16a shows nanoscopic holes in a continuous gold film produced simply by evaporation of gold onto the non-close-packed monolayer and subsequent removal of the colloids. Figure 5.2.16b shows arrays of gold nanocrescents that are produced by evaporation of gold under an angle of $30^{\circ}$ with respect to the surface normal followed by reactive ion beam etching normal to the surface. ${ }^{[136]}$ A more detailed description of the fabrication process and the optical properties of these structures will be presented in chapter 5.3.2.1. Figure 5.2.16 presents arrays of gold nanodiscs that are produced by assembly of a monolayer onto a continuous gold film. After plasma induced size reduction, reactive ion beam etching is used to remove the gold not shaded by the colloids. Finally, gold nanorings can be produced as well (Figure 5.2.16d). Ring formation is achieved by multiple evaporation of gold with an angle of $30^{\circ}$ with respect to the surface normal, followed by reactive ion beam etching similar to the process used for the nanocrescent fabrication. ${ }^{[130]}$

\subsubsection{Ordered binary monolayers with adjustable stoichiometries}

\subsubsection{Introduction}

Beside the non-close-packed architectures described in the previous chapter, a focused research effort has been initiated on binary colloidal monolayers, comprised of a densely packed monolayer of large particles with smaller colloids located in the interstitial sites. In contrast to crystals of uniformly sized particles, such binary architectures feature a rich variety of structures and symmetries depending on the size and number ratios of the large and small colloids applied. They hold promise to be used not only in lithography - with the possibility to create nanostructures not readily available from single-sized particle monolayers - but in several distinct research areas such as crystal surface mimics ${ }^{[104,220]}$ and quasi-crystallinity ${ }^{[221]}$ or, when stacked into layered superstructures, photonic applications aiming at the generation of full photonic bandgap materials. ${ }^{[54,222]}$ One even might envision more complex applications, e.g. for the creation of colloidal molecules by fusing the smaller spheres located in the interstitial sizes. ${ }^{[223]}$ 
For a successful implementation of such binary systems comparable to the wide-spread use of classical, single-sphere type colloidal monolayers, it is of crucial importance to develop reliable and fast crystallization methods that give access to high quality, large area arrays of complex monolayer architectures. However, the growth of binary crystals is substantially more difficult compared to homogeneous colloidal crystals as the presence of small particles requires a tight control over several additional parameters.

Pioneered by $\mathrm{Ozin}^{[110]}$ and van Blaaderen ${ }^{[104]}$ in the last decade, a few methods have been explored for the creation of binary colloidal monolayers, including controlled drying, ${ }^{[104]}$ accelerated evaporation, ${ }^{[110]}$ vertical deposition, ${ }^{[105]}$ spin coating, ${ }^{[109]}$ and electric field induced assembly. ${ }^{[107]}$ A detailed survey of possibilities and limitations is given in chapter 3.3.3.

From a conceptual point of view, gaining precise control over the composition is highly desired to fully exploit the variety of surface structures of two-dimensional binary colloidal crystals. Furthermore, uniform patterning over large areas is very challenging using non-equilibrium processes, as e.g. meniscus pinning and changes in local concentration during the process lead to irregularities during the crystallization. ${ }^{[48,55]} \mathrm{Up}$ to now, precise control over both mentioned features is challenging.

In this chapter, the formation of binary colloidal monolayers at the air/water interface with precisely adjustable compositions over a wide range of size and number ratios using a Langmuir trough is demonstrated. The fraction of the individual spheres at the interface can be determined from the Langmuir isotherms in order to reliably adjust the desired stoichiometry at the interface. Thus, the crystal structure of the binary monolayer can be precisely tailored. After floating and compression, the crystals can be transferred to arbitrary substrates over extended areas only limited by the dimensions of the trough.

\subsubsection{Results and Discussion}

\subsection{Monolayer preparation by Langmuir layer compression and surface lowering transfer}

The process flow for the fabrication of the binary colloidal monolayers is presented in Figure 5.2.17. Before adding the colloidal particles, a transfer substrate was submersed in the trough just below the air/water interface with a slight angle. For the spreading onto the water surface, the colloidal dispersion was diluted in order to obtain a 50 vol.- $\%$ ethanol solution and applied to the air/water interface via a partially immersed hydrophilic glass slide with at a tilt angle of 
approx. $45^{\circ}$ with respect to the water surface following a procedure adapted from Weekes et al. ${ }^{[72]}$ After the colloid deposition was completed, the interface was left to equilibrate for several minutes until no visible movements on the interface were observed before the floating particle layer was compressed. The transfer was typically performed at a surface pressure of $7 \mathrm{mN} \cdot \mathrm{m}^{-1}$ by pumping out the water subphase. In this procedure, the water surface was slowly lowered until the monolayer covered the transfer substrate that was subsequently removed from the trough and dried under an angle of roughly $45^{\circ}$ to facilitate water evaporation. Figure 5.2.17d and e highlight the large area that can be covered with ease. A 6-inch wafer is shown (d) immersed in the water subphase of the trough with an already densely packed monolayer at the interface and (e) after deposition and drying of the monolayer. The monolayer consisted of large particles with a diameter of $1063 \mathrm{~nm}$ and $225 \mathrm{~nm}$ small particles with a number ratio $R_{\mathrm{N}}=$ $N_{\text {small }} / N_{\text {large }}$ of 6 (the corresponding electron microscopy image is shown in Figure 5.2.21e). Individual single domains with square centimeters dimensions are well visible by their distinct colors.
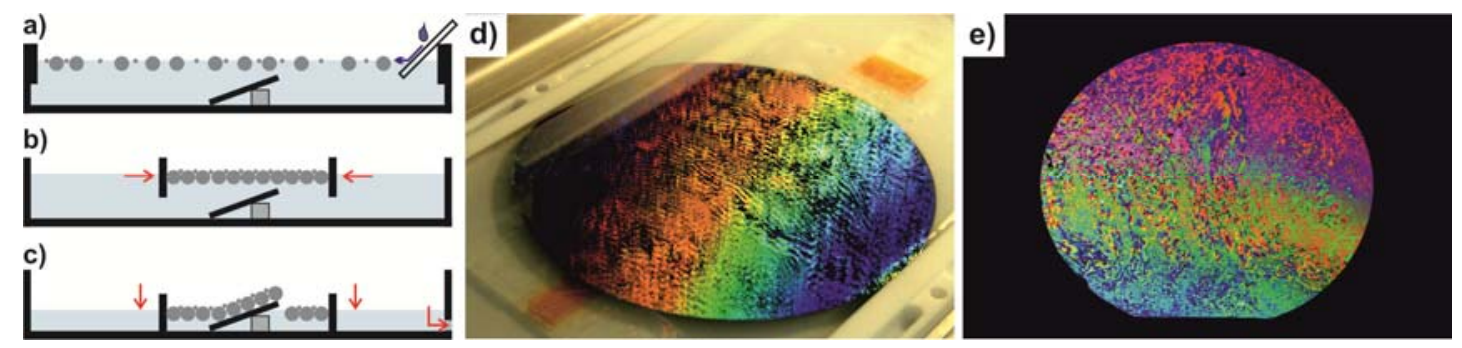

Figure 5.2.17 Illustration of the process flow for the fabrication of large area binary colloidal monolayers and their transfer to a 6-inch silicon wafer: a) Spreading of the colloidal particles at the air/water interface of a Langmuir trough using a glass slide; b) compression of the colloids until a close-packed monolayer is formed; c) lowering of the liquid interface to deposit the monolayer onto a substrate immersed in the subphase; d) wafer substrate immersed in the subphase with a compressed monolayer floating at the

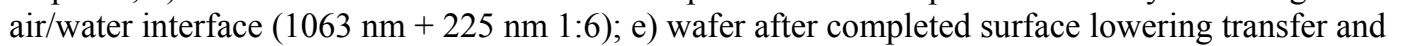
drying. The individual single crystal domains can be distinguished by their coloration.

In order to gain full control over the composition of the binary monolayer, the number ratio of small (S) and large particles (L) has to be properly adjusted. A simple mixing of the two components with the desired ratio is not possible as the individual particles have different tendencies to attach to the interface upon spreading. In other words, only a specific fraction of the spread particles will remain at the air/water interface while the rest will submerge into the water phase. Therefore, the fraction of particles at the interface $(\Phi)$ was individually determined for all colloid types used in this study. To do that, a known concentration of particles (with the amount $\left.N_{\text {total }}\right)$ was spread onto the trough and compressed. The trough area directly before the 
collapse $\left(A_{\text {mono }}\right)$ was determined from the isotherm. This surface area was defined to be the area of the complete monolayer comprising all particles present at the air/water interface. Thus, the number of particles at the interface is slightly overestimated due to defects present in a real monolayer (line defects, unoccupied individual sites). The fraction of particles at the interface is thus calculated as:

$$
\Phi=\frac{N_{\text {interface }}}{N_{\text {total }}}=\frac{0.903 \cdot A_{\text {mono }}}{\pi / 4 \cdot d_{\text {coll }}^{2} \cdot N_{\text {total }}}
$$

with $d_{\text {coll }}$ as the particle diameter and $N_{\text {interface }}$ and $N_{\text {total }}$ being the number of particles at the interface and the total number, respectively. The factor 0.903 represents the fraction of total surface area covered by colloids in perfectly ordered, close-packed monolayer. The total number of colloids added is readily available from the particle concentration and the volume of the dispersion added to the trough. With knowledge of the interface fraction $\Phi$, the desired stoichiometry at the interface can be precisely adjusted. Table 5.2.3 presents the interfacial fractions calculated for all colloids applied in this study for $\mathrm{pH}$ values of the water subphase of 6 and 9 as well as the number of charges per $\mathrm{nm}^{2}$ of the individual colloids at the two $\mathrm{pH}$ values. As will be discussed in detail below, the $\mathrm{pH}$ of the subphase influences the behavior of the colloids at the interface. Carboxylic acid groups, present at the each particle's surface, are increasingly deprotonated at higher $\mathrm{pH}$, thus increasing the hydrophilicity of the particles, leading to a decrease of the interfacial fraction of the colloids. Consequently, the number of charges per $\mathrm{nm}^{2}$, as determined from titration against an oppositely charged polyelectrolyte, ${ }^{[224]}$ increases with increasing $\mathrm{pH}$ for all different colloidal particles used in this study (Table 5.2.3). The charge density is in good agreement with values reported in literature. ${ }^{[225-227]}$

Table 5.2.3. Interfacial fraction and number of charges at a water subphase $\mathrm{pH}$ of 6 and 9 for all colloids used in this study.

\begin{tabular}{ccccc}
\hline Diameter & \multicolumn{2}{c}{ Interface fraction } & Number of charges $/ \mathbf{n m}^{2}$ of particle \\
& & & \multicolumn{2}{c}{ surface } \\
[nm] & $\mathbf{p H 6}$ & $\mathbf{p H 9}$ & $\mathbf{p H 6}$ & $\mathbf{p H 9}$ \\
\hline $202 \pm 8$ & $57.2 \%$ & $27.2 \%$ & 0.8 & 1.1 \\
$225 \pm 7$ & $77.0 \%$ & $36.1 \%$ & 0.8 & 1.3 \\
$303 \pm 8$ & $39.0 \%$ & $20.1 \%$ & 0.8 & 0.9 \\
$336 \pm 11$ & $71.1 \%$ & $24.9 \%$ & 1.2 & 1.6 \\
$422 \pm 10$ & $92.1 \%$ & $42.7 \%$ & 1.1 & 1.3 \\
$1063 \pm 20$ & $98.6 \%$ & $92.2 \%$ & 3.1 & 5.0 \\
\hline
\end{tabular}


In agreement with the higher hydrophilicity of the colloids induced by the higher charge density, the interfacial fraction is decreased by roughly a factor of two when changing the $\mathrm{pH}$ from 6 to 9; the only exception are the large $1063 \mathrm{~nm}$ particles, which showed a very high fraction at the interface for both $\mathrm{pH}$ values. No particular trend is observed concerning the values of the interfacial fractions for the differently sized particles, thus underlining the importance of the accurate determination of the interface fraction for all particles at all different experimental conditions.

\subsection{Surface pressure-area isotherms}
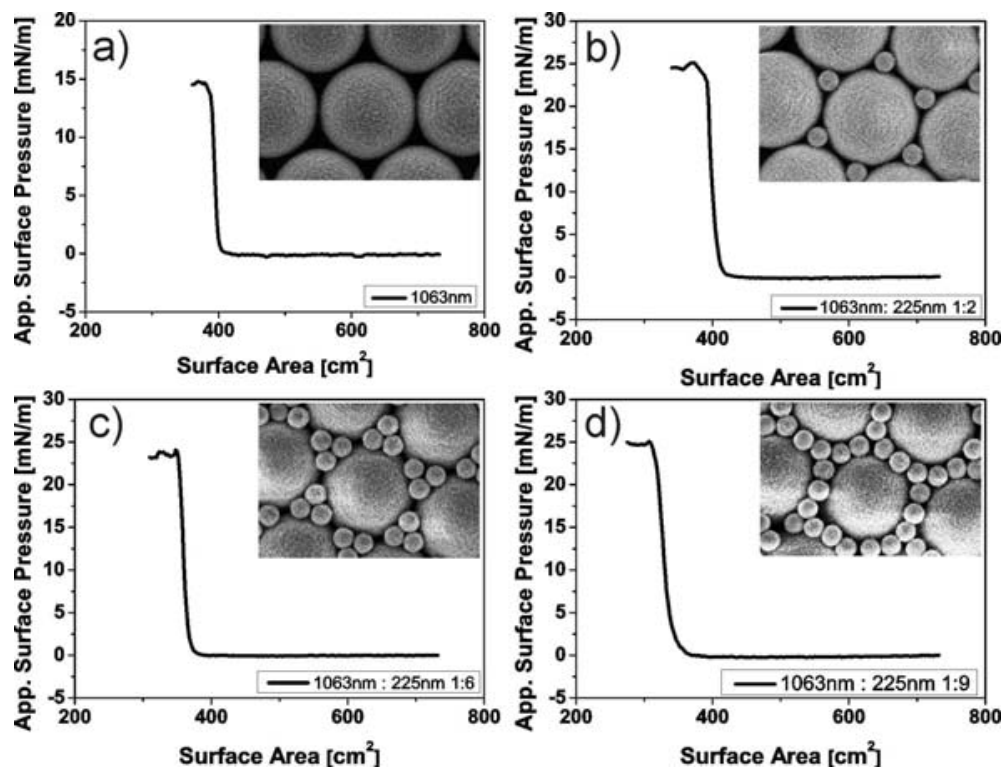

Figure 5.2.18. Surface pressure - area isotherms of single-sized particle- and binary monolayers consisting of $1063 \mathrm{~nm}$ and $225 \mathrm{~nm}$ colloids with different number ratios. a) pure large particles; b) ratio $N_{\text {small }} / N_{\text {large }}=2 ;$ c) ratio $N_{\text {small }} / N_{\text {large }}=6 ;$ d) ratio $N_{\text {small }} / N_{\text {large }}=9$. The insets show electron microscopy images with the respective packing structures.

Surface pressure-area isotherms were recorded for the binary colloidal monolayers. Figure 5.2.18 depicts typical examples, where the isotherms were recorded for the compression of a monolayer of pure $1063 \mathrm{~nm}$ colloids and mixtures of the former with $225 \mathrm{~nm}$ particles in number ratios of 2, 6 and 9. Representative electron micrographs of the monolayer configuration are shown as insets. In general, the shape of the isotherms exhibits a very steep, almost vertical surface pressure increase before the collapse. This behavior is typical for a very stiff film at the interface and is in good agreement with a well-ordered particle monolayer of low compressibility. ${ }^{[77-78]}$ The isotherms of the different monolayers resemble each other very closely, indicating that the interfacial properties are dictated by the large particles. Apparently, a 
dense packing of the large particles is retained for all applied stoichiometries and the small particles are indeed located in the interstitial sites of the large particles. A minor change of the slope is found for the highest number ratio (Figure 5.2.18), reflecting a slightly increased compressibility; even though the slope is still very steep. This behavior hints at a somewhat lower order and is in agreement with a higher amount of defects present in this particular monolayer. ${ }^{[78]}$ Such defects can be compressed to some extend before buckling occurs at the collapse. Hence, the recording of the isotherm provides a convenient way of quality control for the monolayer formation.

\subsection{Theoretical considerations}

First, simple geometric models were applied in order to determine the range of size ratios $R_{\mathrm{S}}=d_{\text {small }} / d_{\text {large }}$ of small particles that can be co-crystallized with the method described. In this respect, two points should be noted. First, it is assumed that highly symmetric, crystalline binary monolayers can only be obtained as long as the large particles form a densely packed monolayer that provides the interstitial sites to be occupied by the small spheres. Second, as will be shown further below, it is possible to control the contact angle of the particles at the air/water interface by adjusting the $\mathrm{pH}$ value. The lowering of the contact angle (and by this, maximizing the degree of immersion into the water subphase) can be exploited to increase the volume of the interstitial sites available at the air water interface (see Figure 5.2.23).

\section{Geometrical calculation of the minimum size of the small particles}

The minimum size of the small particles can easily be determined by considering that for a successful transfer of the binary monolayer, it is necessary that the small colloids are sufficiently large as not to fall through the interstitial sites of the monolayer formed by the large colloids. Hence, they need to be larger than the size of a colloid that fits ideally into the interstices. The size of the colloids that fit ideally into the holes is calculated based on simple geometrical considerations shown in Figure 5.2.19. 


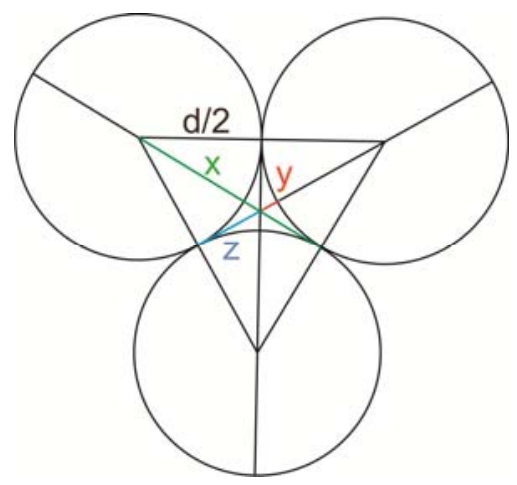

Figure 5.2.19. Sketch to describe the geometrical situation in a close packed monolayer to calculate the minimum size of the small colloids. All variables used for the calculation are defined in the picture. The calculated diameter provides the lower limit for the size of the small colloids. Any colloids with a size bigger than the ideal fitting size will reside on the monolayer of large spheres as they are too big to fall into the interstitial sites. The contact angle of the large colloids is taken as $90^{\circ}$.

Using Pythagoras, $x$ can be calculated from the diameter of the large spheres as:

$$
\begin{gathered}
x^{2}+\left(\frac{d}{2}\right)^{2}=d^{2} \\
x=\left(d^{2}-\frac{d^{2}}{4}\right)^{1 / 2}=\left(\frac{3}{4} d^{2}\right)^{1 / 2}=\frac{\sqrt{3}}{2} d
\end{gathered}
$$

The radius $y$ of the smaller spheres that fit into the site, contributes to $x$ in the following way:

$$
\begin{aligned}
& x=\frac{d}{2}+y+z \\
& z=x-y-\frac{d}{2}
\end{aligned}
$$

Using Pythagoras again, a relation for $z$ and $y$ is given by:

$$
\begin{gathered}
\left(\frac{d}{2}\right)^{2}+z^{2}=\left(\frac{d}{2}+y\right)^{2} \\
\left(\frac{d}{2}\right)^{2}+\left(x-y-\frac{d}{2}\right)^{2}=\left(\frac{d}{2}+y\right)^{2} \\
x^{2}-x d-2 x y+\frac{d^{2}}{4}=0 \\
y=\frac{1}{2 x}\left(x^{2}-x d+\frac{d^{2}}{4}\right)
\end{gathered}
$$


Finally, the minimal size ratio $R_{\mathrm{S}, \min }$ can be determined:

$$
R_{S, \min }=\frac{d_{\text {small }}}{d_{\text {large }}}=\frac{2 y}{d}=\frac{2}{\sqrt{3}}-1 \approx 0.16
$$

For the large colloids $(d=1063 \mathrm{~nm})$ applied for this study, the ideal diameter for the small spheres to match the interstitial site is calculated to be $165 \mathrm{~nm}$.

\section{Geometrical calculation of the maximum size of the small particles}

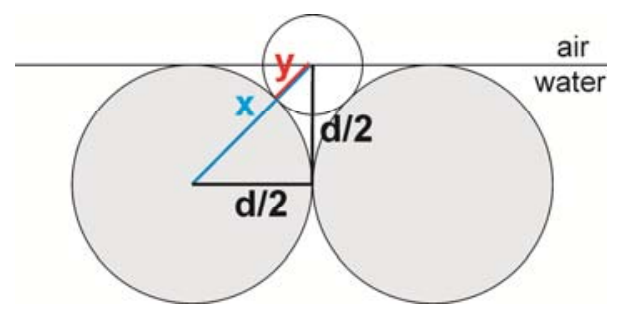

Figure 5.2.20.Sketch of the large particles at the air water interface for the determination of the maximum size of the small particles.

For the determination of the maximum size of the colloids, several assumptions have to be made. As stated above, it is assumed that ordered, crystalline binary monolayers can only be produced as long as the close packed order of the large colloids remains intact during the assembly process. If the small particles forming the superlattice are too large, they will not fit into the sites provided by the underlying large-sphere monolayer and thus, disturb the crystallization process. A disordered, amorphous arrangement will be formed. For the estimation of the theoretical maximum possible size for the small colloids, a contact angle of zero degrees $\left(\theta=0^{\circ}\right)$ for the large particles is assumed; providing the maximal space in the interstitial sizes at the interface while the contact angle of the small particles is fixed to $90^{\circ}$ as shown in the sketch of Figure 5.2.20. With simple geometrical considerations, the maximum size Ratio, $R_{s, m a x}$ is calculated as follows.

Using Pythagoras' theorem, $x$ can be calculated as:

$$
x=\left(\frac{d^{2}}{4}+\frac{d^{2}}{4}\right)^{1 / 2}=\frac{d}{\sqrt{2}}
$$


The radius $y$ of the small particles, can thus be expressed as:

$$
y=\frac{d}{\sqrt{2}}-\frac{d}{2}=d\left(\frac{2-\sqrt{2}}{2 \cdot \sqrt{2}}\right)=0.207 \cdot d
$$

The maximum size ratio can then be determined as:

$$
R_{S, \max }=\frac{2 y}{d_{\text {large }}}=0.41
$$

For the large colloids $(d=1063 \mathrm{~nm})$ applied for this study, the maximum size for the small spheres is calculated to be $440 \mathrm{~nm}$. Note that the calculated value overestimates the maximum diameter for the following reasons. i) The contact angle of the large particles will by slightly higher than 0 degrees, therefore reducing the size of the interstitial site at the interface available for the small colloids. ii) The change in $\mathrm{pH}$ affects the small particles as well. Tuning the $\mathrm{pH}$ value in a way that the large particles are immersed to a high extent (i.e. having low contact angles) in order to provide a maximum space for the small particles will lower the contact angle of the smaller particles as well (as they feature similar surface chemistry). As the small particles lower their contact angle, they will occupy more space in the interstitial site thus leading to a slightly reduced maximum size.

\subsection{Structural diversity}

To assess the possibilities and limitations of the method experimentally, a survey of possible structures was obtained by systematic variation of the size ratio $R_{\mathrm{S}}$ between small (S) and large particles (L) as well as the number ratio $R_{\mathrm{N}}$ of small particles relative to large particles at the air/water interface. Figure 5.2.21 presents electron micrographs of the different binary crystals that can be produced by our approach. Three distinct configurations of the small spheres were formed by changing the number ratio between 2 and 9 (Figure 5.2.21 a to f, from left to right). An interfacial ratio of 2 leads to crystals with one single small particle present in the interstitial site (most conveniently denoted as $\mathrm{LS}_{2}$ ) as this space is energetically most favorable for the small sphere to reside. Increasing the number ratio at the interface to 6 gives rise to a triplet filling of the site upon formation of a $\mathrm{LS}_{6}$ configuration. The side-view image in Figure 5.2.21(1) proves the presence of three particles so that a tetrahedron-like filling with 4 small colloids can be excluded. Finally, a further increase of the number of small particles generates a third distinct phase, the $\mathrm{LS}_{9}$ configuration. In this crystal structure, the interstitial sites are still 
filled with triplets and additional small spheres are located at the bridges between the large particles, as these are energetically the second most favorable region. The monolayers generally featured a high degree of crystallinity as can be observed in the SEM images of Figure 5.2.21. A characteristic lower magnification image, selected for the $\mathrm{LS}_{6}$ configuration of $1063 \mathrm{~nm}+$ $202 \mathrm{~nm}$ is presented in (i) to further demonstrate the high degree of long range order of the monolayers produced.

The particle size ratio was varied between 0.19 and 0.40 (Figure 5.2.21, from top to bottom). Three major differences were observed. For small size ratios $\left(R_{\mathrm{S}}=0.19\right.$ and 0.21$)$, all three configurations $\left(\mathrm{LS}_{2}, \mathrm{LS}_{6}, \mathrm{LS}_{9}\right)$ can be produced in a controlled way. Starting from a size ratio of 0.29 , the small particles are too large to form the $\mathrm{LS}_{9}$ structure. Hence, a number ratio higher than 6 induces a loss of order in the monolayer. The $\mathrm{LS}_{6}$ structure can still be produced and consist of triplets that are already touching each other at the bridges (Figure 5.2.21h). A further increase of the size ratio to 0.32 renders the small particles too large to crystallize in the $\mathrm{LS}_{6}$ structure; only single particles can be placed in the interstitial sites $\left(\mathrm{LS}_{2}\right.$, see Figure 5.2.21i). Any additional small particles compromise the order and induce amorphous structures. Finally, with a size ratio of 0.40 , a surprising arrangement is detected. The monolayer already exhibits a high degree of disorder as the small particles start to prevent the crystallization of the large spheres. Therefore, this size ratio can be considered to be the upper limit for the method presented. However, some small crystalline domains still exist. Instead of forming the expected symmetric $\mathrm{LS}_{2}$ configuration; these domains exhibit a lower symmetry as the small particles at the interstitial sites mutually touch each other and form dimeric structures (Figure 5.2.21k). We attribute this behavior to the small colloids, which because of their size do not sit as firmly in the interstices compared to smaller particles.

The above survey of possible structures demonstrates that the theoretically predicted range of size ratios can be experimentally realized almost completely. 

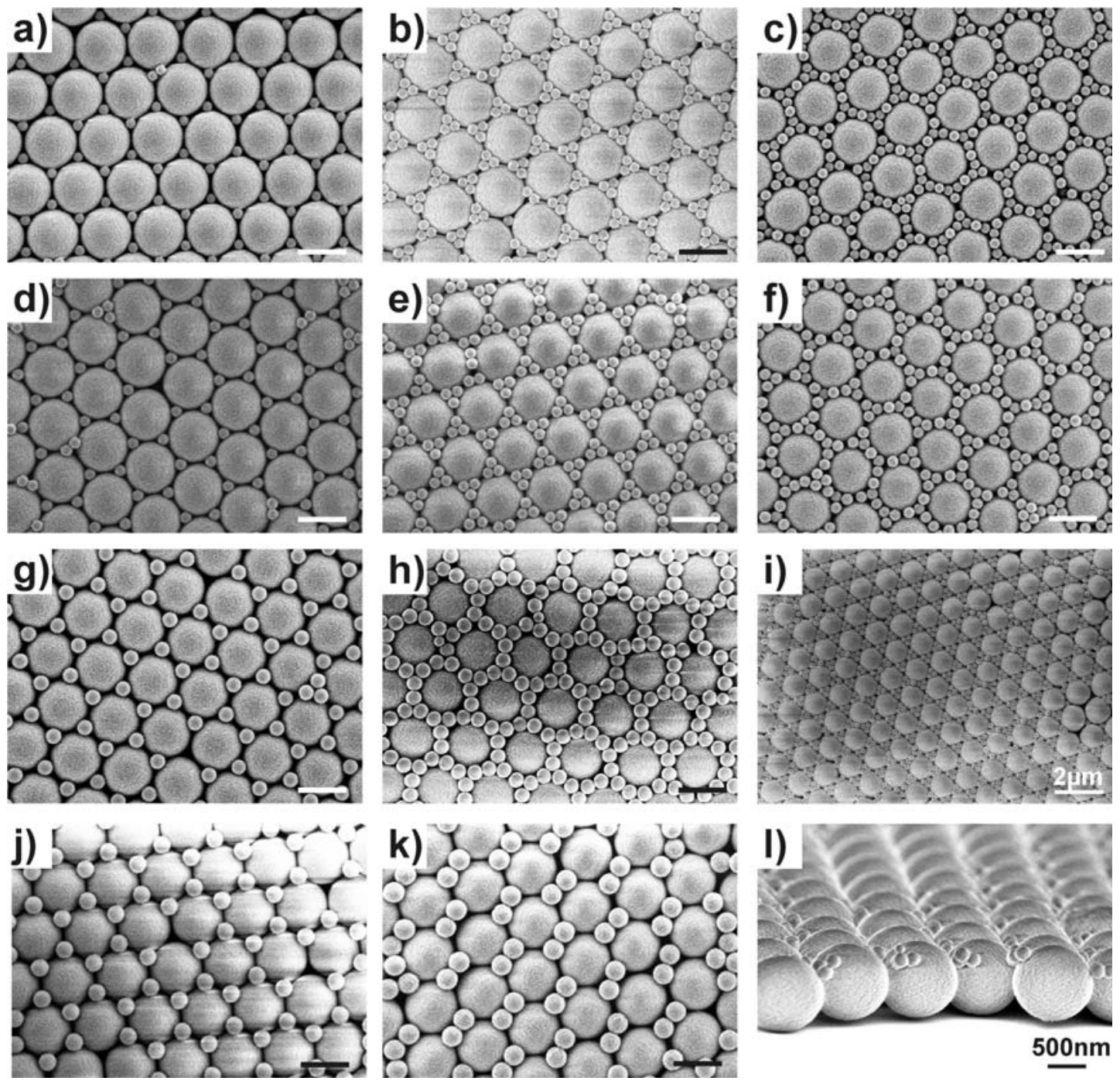

Figure 5.2.21. Structural variety obtained by adjusting the size ratio $R_{S}=d_{\text {small }} / d_{\text {large }}$ and number ratio $R_{N}=N_{\text {small }} / N_{\text {large }}$. The large particles always had a diameter of $1063 \mathrm{~nm}$. The scale bar is $1 \mu \mathrm{m}$ unless otherwise specified. a-c) $R_{S}=0.19\left(d_{\text {small }}=202 \mathrm{~nm}\right)$ and $R_{N}=2(\mathrm{a}) ; 6(\mathrm{~b})$ and $\left.9(\mathrm{c}) ; \mathrm{d}-\mathrm{f}\right) R_{S}=0.21$ $\left(d_{\text {small }}=225 \mathrm{~nm}\right)$ and $R_{N}=2(\mathrm{~d}) ; 6(\mathrm{e})$ and $\left.9(\mathrm{f}) ; \mathrm{g}-\mathrm{h}\right) R_{S}=0.29\left(d_{\text {small }}=303 \mathrm{~nm}\right)$ and $R_{N}=2(\mathrm{~g})$ and $6(\mathrm{~h})$; j) $R_{S}=0.32\left(d_{\text {small }}=336 \mathrm{~nm}\right)$ and $\left.R_{N}=2 ; \mathrm{k}\right) R_{S}=0.40\left(d_{\text {small }}=422 \mathrm{~nm}\right)$ and $\left.R_{N}=2 ; \mathrm{i}-1\right)$ sample shown in b) in lower magnification (i) and in a side-view image (1) to demonstrate that the interstitial sites are indeed covered by triplet structures.

The homogeneity of the interstitial site occupation of the binary monolayers was further quantified statistically and is presented in Table 5.2.4. To do so, several electron micrographs taken from different parts of the surface where examined in detail by systematically analyzing the occupation of the interstitial sites.

Table 5.2.4 shows the statistical analysis of the prepared monolayers. Four distinct occupation scenarios are listed separately: empty interstices, single occupation, triplets, and bridged triplets, corresponding to the crystal structures of L, LS2, LS6 and LS9 respectively. In addition, interstitial fillings not belonging to one of the classes are listed as "others". Typically, these can 
be doublets, tetrahedrons or multiple fillings with additional particles being present on top of a triplet as illustrated in Figure 5.2.22. The letter given behind the value for the percentage of other compositions indicates the predominant type of defect. Care was taken to exclude interstitial sites belonging to defects of the close-packed monolayer of large spheres (e.g. line defects between neighboring domains) to get an accurate picture of the crystalline structure.
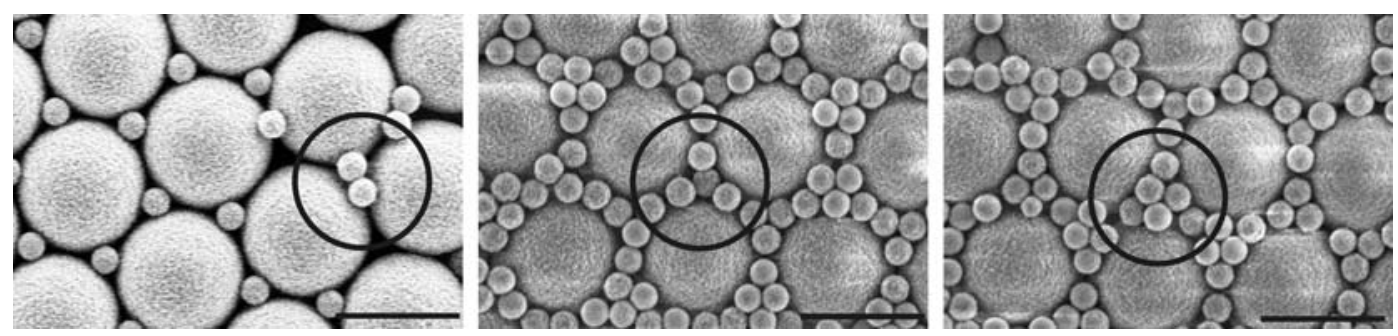

Figure 5.2.22. Additional configurations observed in the binary monolayers. From left to right: double occupation, tetrahedrons and multiple fillings.

Table 5.2.4. Statistical evaluation of the stoichiometries found in the individual binary monolayers with large particles of $1063 \mathrm{~nm}$ diameter.

\begin{tabular}{|c|c|c|c|c|c|c|c|c|}
\hline \multirow{2}{*}{$\begin{array}{l}D_{\text {small }} \\
{[\mathrm{nm}]}\end{array}$} & \multirow{2}{*}{$\begin{array}{c}\text { Ratio } R_{\mathrm{s}} \\
D_{\text {small }}: D_{\text {large }}\end{array}$} & \multirow{2}{*}{$\begin{array}{c}\text { Ratio } R_{\mathrm{N}} \\
N_{\text {small }}: N_{\text {large }}\end{array}$} & \multirow[t]{2}{*}{ pH } & \multicolumn{5}{|c|}{ Interstitial site occupation [\%] } \\
\hline & & & & Empty & Single & Triple & Bridged & Other* \\
\hline 202 & 0.19 & $2: 1$ & 6 & 5.9 & 81.7 & 4.0 & 0 & $8.5(\mathrm{~A})$ \\
\hline 202 & 0.19 & $6: 1$ & 6 & 4.8 & 7.3 & 70.3 & 6.9 & $10.8(\mathrm{~A})$ \\
\hline 202 & 0.19 & $9: 1$ & 6 & 20.8 & 3.9 & 5.8 & 54.1 & $14.9(\mathrm{C})$ \\
\hline 225 & 0.21 & $2: 1$ & 6 & 18.5 & 70.6 & 1.3 & 0 & $9.5(\mathrm{~A})$ \\
\hline 225 & 0.21 & $6: 1$ & 6 & 1.0 & 7.0 & 81.1 & 1.8 & 8.7 (B) \\
\hline 225 & 0.21 & $9: 1$ & 6 & 3.9 & 3.7 & 9.2 & 76.2 & $6.9(\mathrm{~A}-\mathrm{C})$ \\
\hline 303 & 0.29 & $2: 1$ & 6 & 6.1 & 87.3 & 1.2 & 3.3 & $2.1(\mathrm{~A})$ \\
\hline 303 & 0.29 & $6: 1$ & 6 & 10.1 & 9.5 & 64.6 & $3.5^{* *}$ & $11.4(\mathrm{~A})$ \\
\hline 303 & 0.29 & $6: 1$ & 9 & 0.2 & 2.9 & 80.8 & $0.5^{* *}$ & $15.5(\mathrm{~A})$ \\
\hline 303 & 0.29 & $9: 1$ & 6 & 0 & 0 & 74.7 & $0^{* *}$ & $25.3(\mathrm{D})$ \\
\hline 336 & 0.32 & $2: 1$ & 6 & 10.5 & 80.6 & 3.4 & 0 & $5.6(\mathrm{~A})$ \\
\hline 422 & 0.40 & $2: 1$ & 9 & 2.9 & $\begin{array}{c}95.6 \\
(69.5)^{* * *}\end{array}$ & 0 & 0 & 1.4 (D) \\
\hline
\end{tabular}

*The letter in brackets gives the dominating arrangement of small spheres in the interstitial site: A: double; B: Tetrahedron; C: multiple filling, D: amorphous.

**" bridged" here refers to single particles connected by a single particle; to be distinguished from neighboring triplets that directly touch each other as well.

${ }^{* * *}$ Single small particles tended to form dimeric structures as shown in Figure $5.2 .21 \mathrm{k}$. The numbers should be read as follows: $69.5 \%$ of the single-occupied sites were found to be dimers by touching the next single-occupied site. 
In general, the occupation efficiency with the desired configuration was between 70 and $80 \%$. In all cases, deviations from the desired filling occurred, mostly by switching between the energetically most favorable configurations. Given the large number of possible configurations for the small spheres, the values determined for the desired compositions are rather high. This justifies our claim that the composition is controlled by adjusting the number ratio $\left(R_{N}\right)$ of the colloids at the interface.

\subsection{Variation of the $\mathrm{pH}$ value as a means to influence the crystallization process}

A parameter that can be used to further influence the crystallization process is the $\mathrm{pH}$ value of the subphase. ${ }^{[88]} \mathrm{An}$ increase in $\mathrm{pH}$ can be used to increase the crystallization efficiency into the desired structure. As the particles featured carboxylic acid surface functionalization, an increase in $\mathrm{pH}$ leads to a higher amount of charges at the surface (see Table 5.2.3) as the acid groups are increasingly deprotonated. This has several effects on the monolayer crystallization process. First, the increase in electrostatic repulsion helps to increase the long range order in the monolayer system ${ }^{[88]}$ as attractive van der Waals and capillary forces are counteracted more efficiently and the particles have more time to find the energetic minimum position. ${ }^{[38,55]}$ Moreover, the higher charge density increases the hydrophilicity of the colloids with two consequences. First, the higher particle solubility in the subphase is reflected by a drop in the interfacial fraction for all colloids (Table 5.2.3). Hence, the interfacial fraction has to be determined separately for different $\mathrm{pH}$ values. Second, due to the higher charge density, the particles floating at the interface will tend to be immersed deeper into the subphase (i.e. the contact angle will change to lower values). Figure 5.2.23 illustrates this phenomenon graphically. As the large particles sink deeper into the water subphase, the interstitial volume at the interface becomes larger. Thus, larger particles can fit more easily into the crystal structure of the monolayer. In order to experimentally verify the dependence of the contact angle on the $\mathrm{pH}$ value, $\mathrm{n}$-butyl cyanoacrylate was polymerized at the interface in order to fix the position of the colloids at the air/water interface in a polymeric matrix. The monomer is introduced slowly via the gas phase, to keep the monolayer and the subphase surface as unperturbed as possible. As the cyanoacrylate polymerization is initiated by water, the polymer film selectively grows into the water phase. Thus, the position of the colloids at the liquid interface can be visualized (preparation scheme is shown in the experimental part in chapter 6.5.3.4. Figure 5.2.23c and d shows electron micrographs of a monolayer of large colloids embedded in the poly(n-butyl cyanocrylate) (PBCA) film at a pH of 6 and 9, respectively. A clear change in contact angle can 
be seen with the colloids at $\mathrm{pH}=9$ being almost completely immersed in the water subphase. Hence, the interstitial volume available for the small colloids is drastically increased compared to the situation at $\mathrm{pH}=6$. This was exploited for the crystallization of binary monolayers with large size ratios $\left(R_{\mathrm{S}}=0.32\right.$ and 0.40$)$. The occupation with single particles $\left(R_{\mathrm{S}}=0.32\right)$ was thus increased from 65 to $81 \%$ (Table 5.2.3) and the order in the film was substantially higher. Figure 5.2.23e and $\mathrm{f}$ compares the monolayers formed with a size ratio of 0.40 for a $\mathrm{pH}$ of 6 and 9. Crystalline domains formed exclusively with a $\mathrm{pH}$ of 9 , even though the domains were small and the monolayer featured a higher amount of disorder compared to smaller size ratios. Using pure water ( $\mathrm{pH} 6$ ), only amorphous structures were obtained as the crystallization of the large particles was impeded by the large diameter of the small particles at the interface.

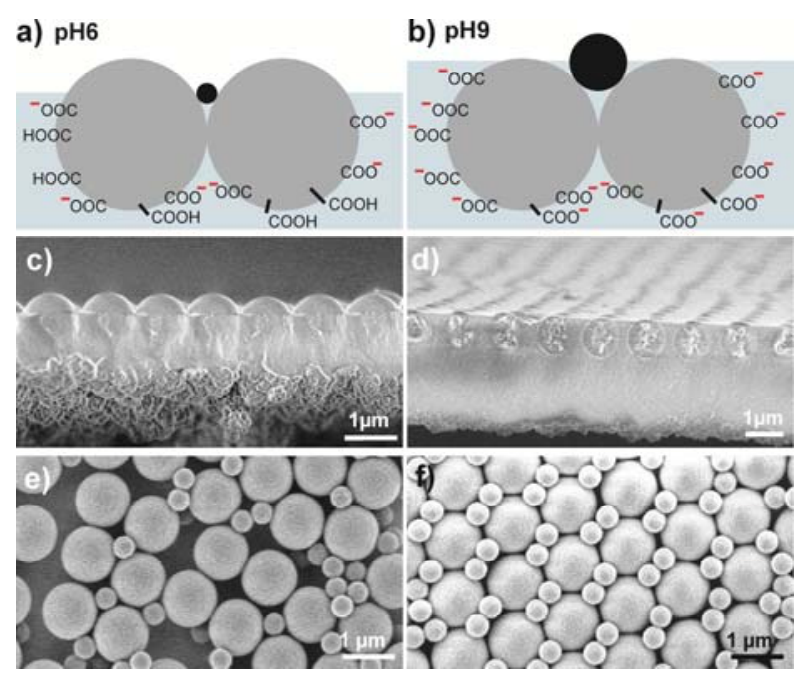

Figure 5.2.23. Effect of the $\mathrm{pH}$ value on the position of the monolayer at the interface and consequences for the crystallization of a binary system composed of $1063 \mathrm{~nm}$ and $422 \mathrm{~nm}$ colloids (size ratio RS $=0.40$ ). a,b) Schematic illustration of one small- between two large colloids at the air/water interface at $\mathrm{pH}=6$ (a) and $\mathrm{pH}=9$ (b). The black sphere illustrates the maximum size of a small colloid that fit into the interstitial site at the interface without disturbing the crystal structure of the large sphere monolayer; c,d) SEM images of a monolayer embedded in a poly-(butyl cyanocrylate) matrix that was polymerized at the interface to visualize the colloid positions at $\mathrm{pH} 6(\mathrm{c})$ and $\mathrm{pH} 9(\mathrm{~d})$; e,f) binary monolayers with a size ratio of 0.40 produced at $\mathrm{pH} 6(\mathrm{e})$ and $\mathrm{pH} 9$ (f). 


\subsection{Transfer to different substrates}

In addition to the control over monolayer composition, the surface lowering transfer technique has another distinct benefit as it can be applied to a large variety of substrate types. The only requirement for a substrate is that is must be sufficiently hydrophilic in order not to completely dewet during the deposition process. However, even hydrophobic substrates can be used if they are treated with oxygen plasma for a short time prior to transfer. Planarity of the surface is generally not a requirement for the deposition process, as documented in Figure 5.2.24 $\mathrm{c}$ to $\mathrm{g}$.

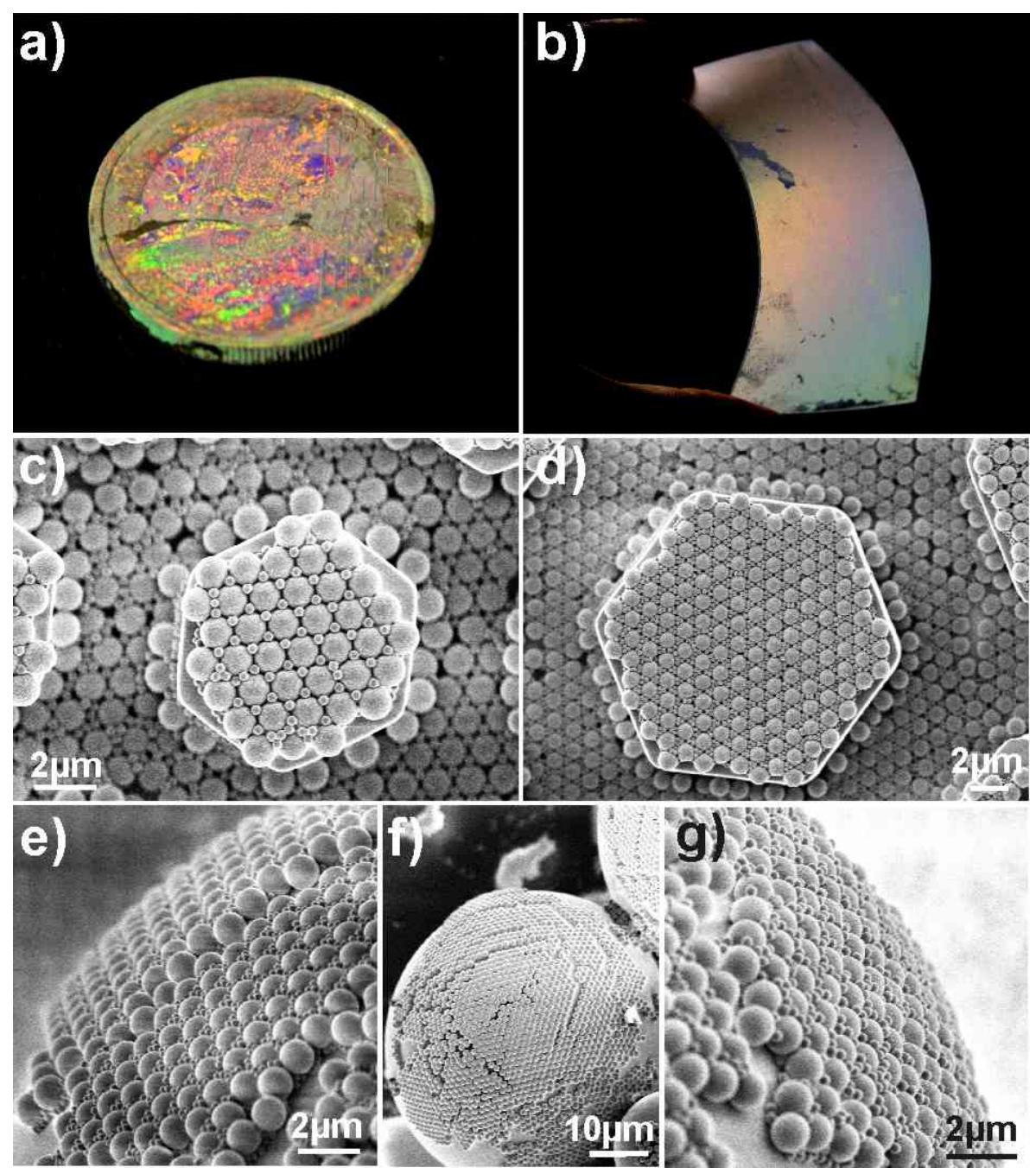

Figure 5.2.24. Examples of substrates that can be conveniently decorated with binary colloidal monolayers by the method presented. a) One-euro coin; b) Plastic substrate; c,d) micropillars of photoresist created by conventional photo-lithography covered with $1063 \mathrm{~nm}+303 \mathrm{~nm}$ single particles (c) and $1063 \mathrm{~nm}+225 \mathrm{~nm}$ triplets (d); e-g) Binary monolayers transferred onto $50 \mu \mathrm{m}$ polystyrene particles; e-f) $1063 \mathrm{~nm}+225 \mathrm{~nm}$ triplets; g) $1063 \mathrm{~nm}+303 \mathrm{~nm}$ triplets. 
Figure 5.2.24 provides examples of substrates patterned with binary monolayers. Macroscopically, different objects of (almost) any material and shape can be decorated with the binary colloid structures as demonstrated by a one-euro coin (a) and a bendable poly ethylene substrate (b). In the latter case, the plastic substrate was hydrophobic, but a short oxygen plasma treatment induced enough hydrophilicity to prevent dewetting of the substrate during transfer. Substrates featuring micropillars of photoresist prepared by conventional photo-lithography can be precisely covered with binary monolayers as well (c,d). Finally, $50 \mu \mathrm{m}$ polystyrene particles were deposited onto a silicon wafer and subsequently decorated with binary monolayers of $1063 \mathrm{~nm}+225 \mathrm{~nm}$ or $303 \mathrm{~nm}$ (Figure 5.2.24e and f). As can be seen in the lower magnification image of Figure 5f, the upper half of the colloid surface, which is accessible during the surface lowering transfer, is completely covered with the monolayer. The monolayer adheres even in the very steep regions around the large particle's equator. The high quality of the monolayer is highlighted in the higher-magnification images of Figure 5.2.24e and g.

\subsection{Applications in Nanosphere Lithography}

Using the binary monolayers as evaporation masks, a possible application of the binary surface structures can be demonstrated. In conventional colloidal lithography, ${ }^{[5]}$ using single-size sphere monolayers as evaporation masks, arrays of gold nanotriangles can be prepared by gold evaporation. They possess well defined plasmon resonances ${ }^{[125]}$ and have thus been used extensively e.g. in sensing applications. ${ }^{[124,150]}$ However, the architecture of the monolayer limits this process to triangular structures that reflect the projection shape of the interstitial sites and their hexagonal arrangement. The interparticle distance of the evaporated nanostructures as well as their dimensions are simultaneously determined by the size of the colloids used as the monolayer mask. By controlled filling of the interstitial locations with smaller particles a superstructure is imposed with an increase in the area shaded by the colloids during the gold evaporation process. Hence, the dimension and the separation of the evaporated nanostructures are decoupled and more complex surface patterns can be generated.

Figure 5.2.25 presents examples of such patterns produced. In general, two types of available patterns arising from $\mathrm{LS}_{2}$ and $\mathrm{LS}_{6}$ binary structures may be of particular relevance. Using monolayers with a $\mathrm{LS}_{6}$ configuration yields relatively densely packed monolayer architectures as the triangles of small spheres in the interstitial sites occupy most of the free space available. However, the small spheres feature one unoccupied free site in the center of the triangle, which itself is centered in the interstitial site between the large spheres. After evaporation and colloid removal, this architecture produces arrays of very small gold nanotriangles with a large spacing 
(independent control over size and separation). Figure 5.2.25a and b show examples of such surface patterns.
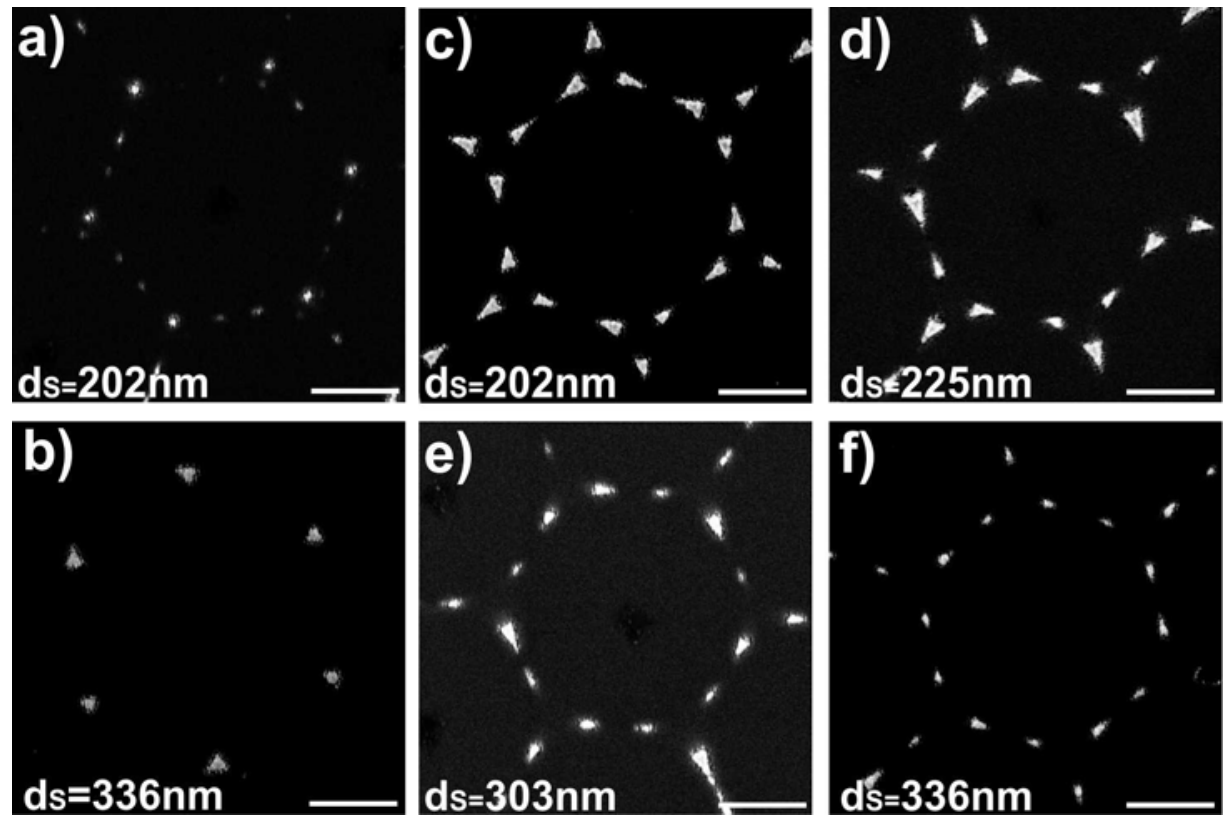

Figure 5.2.25. SEM images of gold nanostructures produced by different binary monolayer architectures as colloidal lithography masks. The size of the large colloids was $1063 \mathrm{~nm}$ in all cases. The size of the small colloids $d_{S}$ is denoted in each image. The scale bars are $400 \mathrm{~nm}$; a,b) LS $_{6}$ structures used as mask; c-f) $\mathrm{LS}_{2}$ structures used as mask.

Figure 5.2.25a shows gold structures produced with a $\mathrm{LS}_{6}$ structure of $1063 \mathrm{~nm}$ and $202 \mathrm{~nm}$ colloids. A hexagon of gold dots reflecting the free interstices of the small particles is clearly visible. The size of the dots is approximately $35 \mathrm{~nm}$. The triangular structure is almost completely lost, most likely due to the small size and the rather large distance between mask and substrate. Additional sets of smaller dots appear between the hexagonal dots that stem from empty space between the edges of the triangles and the area where the large colloids are touching. In Figure 5.2.25b, a LS $_{6}$ structure of $1063 \mathrm{~nm}$ and $336 \mathrm{~nm}$ colloids was used as mask. The triangular shape of the gold particles is now clearly visible. No further gold dots appear as the triangles of the small particles cover all empty space of the large colloid monolayer except for their own interstitial site. The size of the triangles (edge to edge distance) is approximately $50 \mathrm{~nm}$. Their mutual spacing is roughly $650 \mathrm{~nm}$. The small size of the nanostructures paired with a long separation distance meets the key requirement for single particle imaging and spectroscopy, where a minimum separation distance close to the wavelength of the applied light is required. Thus, these surface structures may be valuable substrates for the study of binding events of individual molecules onto single nanostructures. 
Another interesting type of surface patterns is created with monolayers of a $\mathrm{LS}_{2}$ structure. As these crystals feature single occupied interstices, more free space exists in the $2 \mathrm{D}$ crystal as compared to the $\mathrm{LS}_{6}$ structure described above. Figure 6c to $\mathrm{f}$ presents gold nano-structure arrays produced with $\mathrm{LS}_{2}$ monolayers of various size ratios between small and large particles. In all cases, highly complex structures are visible. Generally, they can be considered as variations of the classical triangular structures produced from single particle monolayers. However, as the small particle shades a central part of the triangular interstitial site, triplets of smaller triangles with a local $D_{3 h}$ symmetry are produced. These are arrayed in a $D_{6 h}$ symmetry reflecting the symmetry of the monolayer. Depending on the size of the colloids in the interstices, the size and mutual distance of the individual triangles can be varied drastically. In the structures produced, they varied from $100 \mathrm{~nm}$ sized triangles with $150 \mathrm{~nm}$ spacing produced from $202 \mathrm{~nm}$ colloids in the interstices to $70 \mathrm{~nm}$ triangles with $250 \mathrm{~nm}$ spacing. Recently, arrays of nanoparticle clusters - resembling such arrays of triplet nanotriangles - have attracted significant attention as they are associated with a variety of optical phenomena not occurring in spatially separated objects, most prominently hybridization of the individual plasmon resonances ${ }^{[151-152]}$ and Fano-type resonances. $^{[228-229]}$

\subsubsection{Conclusion}

In summary, a methodology for the fabrication of large areas of binary colloidal monolayers consisting of a densely packed monolayer of large spheres (L) with small spheres (S) located in the interstitial sites is proposed. The occupation of these sites can be tailored and precisely controlled over a wide range of size ratios on a Langmuir trough. A simple theoretical model based on geometrical considerations and taking into account the particle immersion depth (depending on the contact angle) was applied to estimate the range of size ratios that can be applied and yielded possible size ratios of $0.16<R_{\mathrm{S}}<0.41$. Using surface pressure - area isotherms, the fraction of the different colloids at the interface was determined for each type of particle for every experimental condition individually. These data can be used to precisely adjust the exact stoichiometric compositions of the large and small spheres at the interface. Crucial to yield highly crystalline monolayers is the spreading technique via a tilted glass slide with ethanol/water mixtures. Apparently, the particles pre-organize at the interface and form monolayers with large domain sizes upon complete compression. By variation of the interface stoichiometry, highly ordered binary monolayers with crystal structures of $\mathrm{LS}_{2}, \mathrm{LS}_{6}$ and $\mathrm{LS}_{9}$ were successfully prepared. Depending on the size ratio, either all three configurations $\left(R_{\mathrm{S}}<0.22\right)$, only $\mathrm{LS}_{2}$ and $\mathrm{LS}_{6}$ structures $\left(R_{\mathrm{S}}=0.29\right)$ or only $\mathrm{LS}_{2}$ crystals $\left(0.30<R_{\mathrm{S}}<0.40\right)$ were 
formed. A statistical analysis of the occupation of the interstitial sites of the monolayer of large spheres was performed and revealed a high degree of order with usually $70-80 \%$ prevalence of the target configuration. An optimization of the preparation conditions, e.g. automatic spreading, vibration damping of the trough or optimized protocols for preassembly time and compression speed is expected to further increase the yield of the desired structural types. The $\mathrm{pH}$ value of the water subphase can be used to tune the crystallization process as the contact angle of the large spheres at the interface strongly depends on the $\mathrm{pH}$ value, thus changing the interstitial volume available for the smaller colloids.

An interfacial polymerization of n-butyl cyanoacrylate was used to directly visualize the position of the colloids at the interface. The compressed binary monolayers were sufficiently robust to allow transfer to a variety of substrates of various materials even with non-planar surfaces and arbitrary topographic features. The process is fast, easy to implement, does not require expensive equipment and is able to pattern large areas only limited by the troughs dimensions. Thus, we expect the method to be well amenable for industrial applications.

The prepared binary monolayers were used as evaporation masks to generate gold nanostructure arrays by colloidal lithography. Given the complex geometries of the interstitial sites in the binary 2D crystals, more complex arrangements of the nanostructures were prepared. This demonstrates an increase in flexibility for colloidal lithography compared to conventional single-particle monolayers. 


\subsection{Lithographic processes using colloidal monolayers}

\subsubsection{Arrays of metal nanoparticles by a non-conventional lithographic process}

This chapter describes a non-conventional lithographic process to produce arrays of metal nanoparticles from metal-complex containing latex precursors. The process was developed at the University of Ulm and first published by Manzke et al. in 2007. ${ }^{[121]}$

$\mathrm{Pt}(\mathrm{acac})_{2}$ and $\mathrm{Fe}(\mathrm{acac})_{3} / \mathrm{Pt}(\mathrm{acac})_{2}$ containing latex particles, the synthesis of which is described in chapter 5.1.2, are assembled into a close-packed monolayer and subjected to oxygen plasma. The treatment leads to a decrease in size of the particles while their lattice positions remain unaffected. A thermal annealing step finally converts the crude metal containing nanodots into crystalline nanoparticles. A precise control of size and interparticle spacing is demonstrated for arrays of platinum nanoparticles. Using latex particles containing $\mathrm{Fe}(\mathrm{acac}) 3$ and $\operatorname{Pt}(\mathrm{acac}) 2$ in stochiometric ratios gives rise to arrays of magnetic FePt alloy particles. All etching experiments presented here were performed by Achim Manzke at the University of Ulm but are presented as the major application of the metal-containing colloids synthesized as part of this thesis. The results presented in this chapter are published as "Arrays of Size and Distance Controlled Platinum Nanoparticles Fabricated by a Colloidal Method",[230] and "Bimetallic Alloy Nanoparticles Prepared by Miniemulsions". [231] 


\subsubsection{Introduction}

The controlled generation of arrays of inorganic, metallic nanoparticles on solid substrates may be identified as one of the key processes to exploit the benefits of nanomaterials in materials science and technology. In contrast to polymeric latex particles that mostly serve as sacrificial layers in patterning processes, metallic nanoparticles inherently possess a rich variety of physical properties that are either related to the bulk properties of the respective metals or a size-dependent consequence of their small volume and large surface. The former include materials properties such as magnetism or resistance towards reactive ion etching processes while one of the most prominent examples of the latter are high catalytic activities. ${ }^{[232]}$

For several applications, the placement of such nanoparticles in a symmetric lattice is of crucial importance. As examples, platinum nanoparticles can then serve as etching masks to create highly ordered arrays of silicon nanopillars or nanoholes ${ }^{[121]}$ while gold nanoparticle arrays have been used in plasmonics, ${ }^{[233]}$ catalysis, ${ }^{[232]}$ the generation of protein arrays ${ }^{[234]}$ and to study cellsurface interactions. ${ }^{[16,235]}$ Novel data storage devices might arise from the controlled placement of magnetic nanoparticles in symmetric arrays. As such, the $\mathrm{L} 1_{0}$ phase of iron-platinum alloys has been identified as a promising candidate as it exhibits ferromagnetism down to very small particle sizes. ${ }^{[236]}$

Several techniques to prepare such arrays have been demonstrated and were introduced in chapter 3.4. They can roughly be divided into processes that use nanoscale patterning methods to create masks that are subsequently used to pattern the desired material and processes that take advantage of precursor materials that are transformed into the respective metal or metal alloy. Colloidal- and block copolymer lithography are archetypal examples of the first group. The second approach was introduced by Spatz et al. ${ }^{[120]}$ who assembled metal-salt containing blockcopolymer micelles on substrates. After applying a plasma etching process, the organic material of the micelle was combusted and arrays of metal nanoparticles formed on the substrate. The technique enabled the formation of nanoparticle arrays with sizes between 1 and $9 \mathrm{~nm}$ and interparticle spacings between $25 \mathrm{~nm}$ and $140 \mathrm{~nm}$.

To increase the order of the system, the micelles were subsequently exchanged for colloidal particles that are able to form monolayers with a higher symmetry byManzke et al. ${ }^{[121]}$ Using miniemulsion polymerization, platinum-acetylacetonate was incorporated into polystyrene particles. To yield metallic nanoparticles, the complex containing latex particles were assembled into monolayers and subjected to a plasma treatment and thermal annealing step as schematically illustrated in Figure 5.3.1. In contrast to classical nanosphere lithography, the colloids in the presented process are not used as masks for the patterning but act as a sacrificial material that is combusted to give rise to the arrays of metal nanoparticles. 


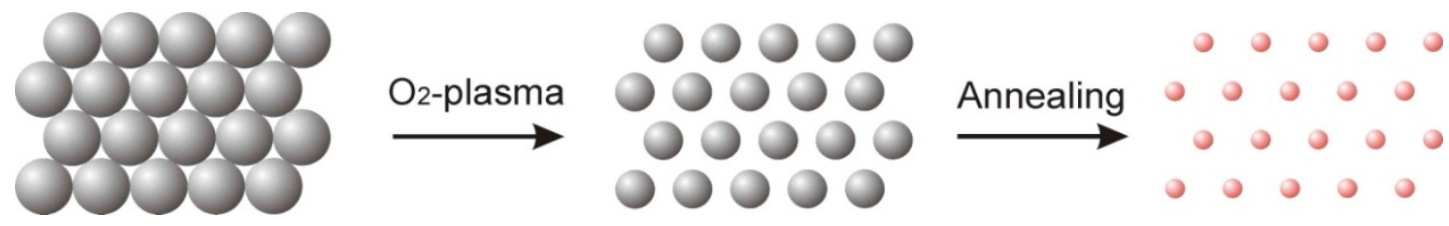

Figure 5.3.1. Schematic illustration of the non-conventional lithography process

As a part of this thesis, the non-conventional lithography process was further refined in cooperation with the solid state physics department of the University of Ulm. They used the metal complex containing nanoparticles, the synthesis of which was presented in chapter 5.1.2 to create metal nanoparticle arrays using the same process as presented in Figure 5.3.1. Two main aspects were in the focus of the project. First, a precise control of the nanoparticle size and interparticle distance was demonstrated for platinum nanoparticle arrays in order to assess sizes and distances accessible by the process. Second, the versatility of the process was investigated by creating nanoparticle arrays of more complex, functional materials. For that, latex particles containing iron(III)acetylacetonate and platinum(II)acetylacetonate simultaneously were successfully transferred into ferromagnetic FePt alloy nanoparticles. Attempts to create gold nanoparticle arrays are under current investigation have not yet been successful, mainly due to the difficulties in finding suitable gold complexes.

\subsubsection{Platinum nanoparticle arrays}

\section{Control over nanoparticle size}

To control the size of the final platinum nanoparticles, emulsion polymerization of styrene was performed with varying amounts of platinum(II)acetylacetonate. The synthesis and characterization of the particles was presented in chapter 5.1.2.2.1 and the most important features of the colloids are summarized in Table 5.3.1. 

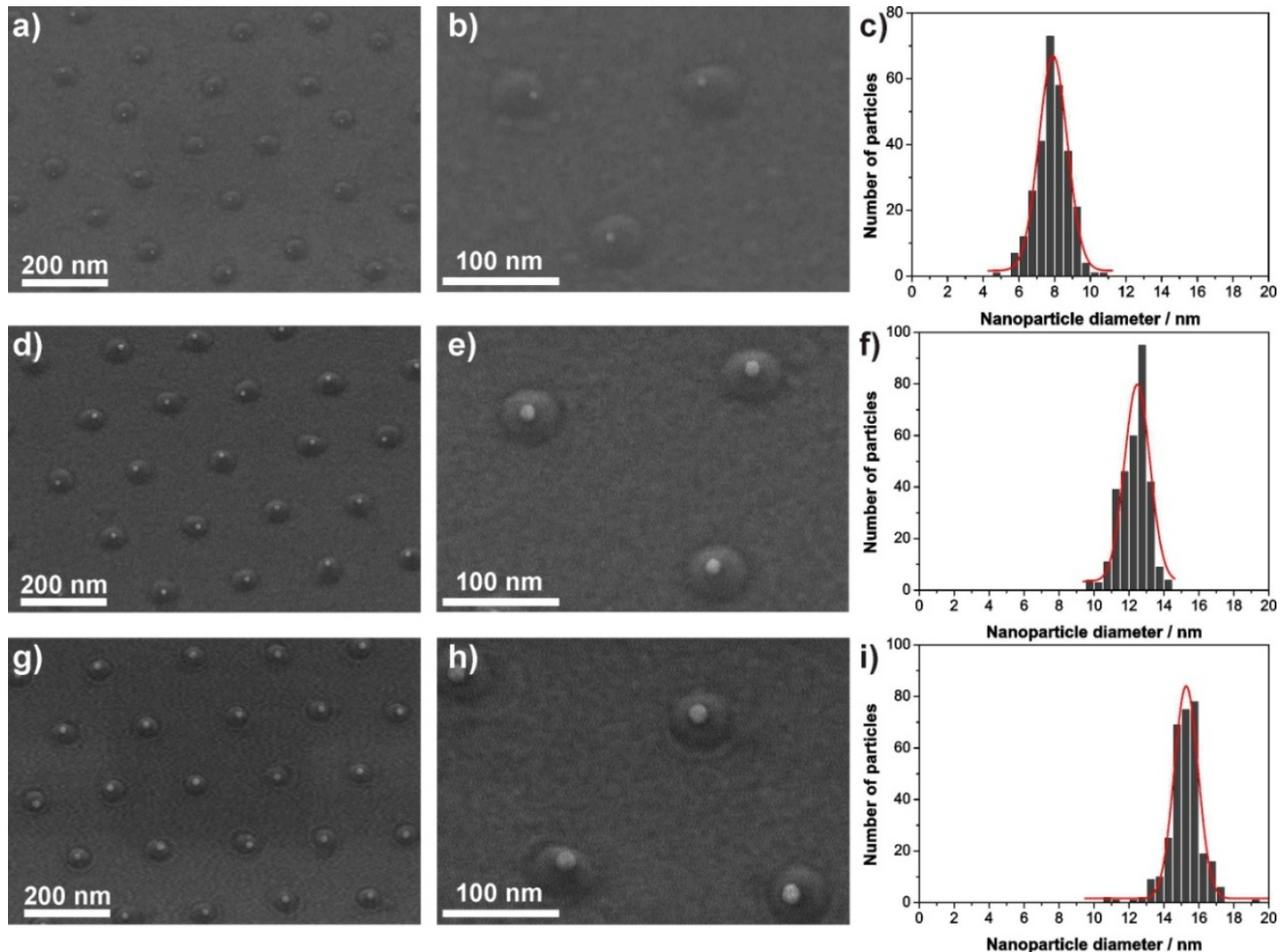

Figure 5.3.2. Control of nanoparticle size in the non-conventional lithography process with platinum(II)acetylacetonate containing latex particles. From top to bottom: colloids containing $0.39 ; 0.75$ and $1.37 \mathrm{wt} .-\%$ of platinum. a,d,g) Low magnification electron microscopy images demonstrating the lateral order in the nanoparticle arrays. b,e,h) High magnification SEM images. c,f,i) Nanoparticle size histograms. Experiments performed and images provided by Achim Manzke; University of Ulm.

Figure 5.3.2 presents the results of the non-conventional lithography process performed by Achim Manzke at the University of Ulm. Three colloid samples with nearly identical diameters but varying metal contents were subjected to the lithography process. First, oxygen plasma was applied in order to combust the organic material. After 60 min of etching time, crude nanodots were formed on the substrate that could not be further reduced in size by plasma treatment. A thermal annealing step $\left(10 \mathrm{~min}\right.$ at $\left.1100^{\circ} \mathrm{C}\right)$ was conducted to transfer these crude compounds into crystalline platinum nanoparticles.

From top to bottom, the amount of $\mathrm{Pt}(\mathrm{acac})_{2}$ in the latex particles increased. A clear increase in size of the resulting metal nanoparticles can be seen. A statistical evaluation of the nanoparticle size (Figure 5.3.2c,f,i) quantifies this increase in size. Table 5.3.1 summarizes the properties of the nanoparticle array. The nanoparticle size was varied between $8 \mathrm{~nm}$ and $15 \mathrm{~nm}$ and only minor deviations from the theoretical value (1-2 nm) occurred. The interparticle distance closely reflects the diameter of the initial colloidal particles and did not differ strongly between the individual samples. The high degree of order can be seen in the electron micrographs of Figure 5.3.2a, $\mathrm{d}$ and $\mathrm{g}$ and is further highlighted by a lower magnified image (Figure 5.3.3), exemplarily chosen for the sample with the highest platinum content. The Fourier transformation, shown as 
inset in the figure, underlines the hexagonal symmetry and long-range order of the nanoparticle array.

Table 5.3.1. Characteristics of latex particles containing different amounts of $\mathrm{Pt}(\mathrm{acac})_{2}$ and resulting nanoparticles after the etching process.

\begin{tabular}{ccccccc}
\hline $\begin{array}{c}\text { Pt(acac) } \\
\text { added }\end{array}$ & \multicolumn{2}{c}{$\begin{array}{c}\text { Pt-content } \\
\text { measured }\end{array}$} & \multicolumn{2}{c}{ Final NP size } & $\begin{array}{c}\text { Diameter } \\
\text { Latex } \\
\text { theoretical } \\
\text { value* } \\
\text { [nm] }\end{array}$ & $\begin{array}{c}\text { Interparticle } \\
\text { Spacing }\end{array}$ \\
$\begin{array}{c}\text { [wt.-\% of } \\
\text { monomer] }\end{array}$ & [wt.-\%] & $\begin{array}{c}\text { [nm] } \\
\text { [\%eory] }\end{array}$ & [nm] & [nm] \\
\hline 1.0 & 0.39 & 69.2 & 10 & $8 \pm 1$ & $175 \pm 7$ & $173 \pm 20$ \\
2.0 & 0.75 & 75.5 & 14 & $12 \pm 1$ & $196 \pm 5$ & $198 \pm 21$ \\
4.0 & 1.37 & 78.5 & 16 & $15 \pm 1$ & $185 \pm 5$ & $190 \pm 14$ \\
\hline
\end{tabular}

*calculated from the metal content of one colloid as determined by ICP-OES.

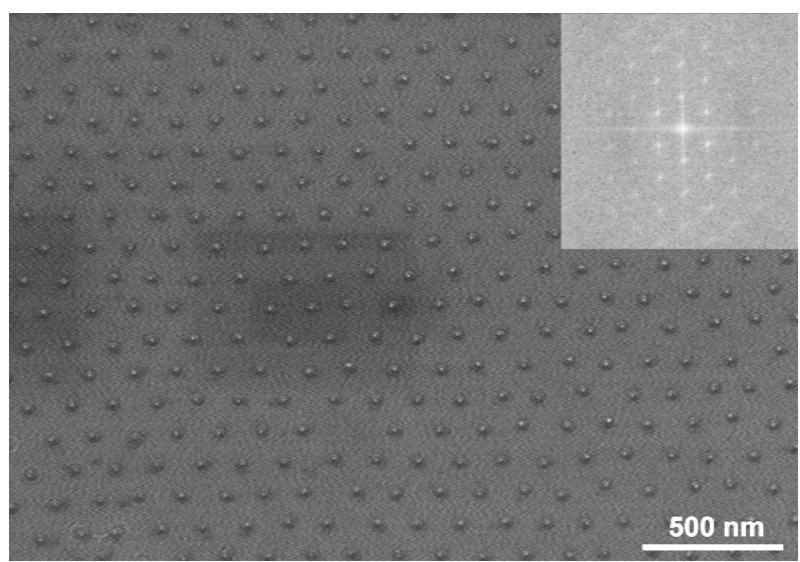

Figure 5.3.3. Lower magnified electron micrograph, exemplarily chosen for biggest platinum nanoparticles demonstrating the high order of the array. The inset shows a Fourier transformation of the image. Image provided by Achim Manzke; University of Ulm.

The metal nanoparticles appear to be sitting in the middle of a circular elevation of the substrate. This elevation is attributed to the increased formation of silicon dioxide in the vicinity of the nanoparticles due to the catalytic activity of platinum in silicon oxidation reactions. ${ }^{[237]}$ Figure 5.3.4 presents an electron microscopy images of a platinum nanoparticle array after treatment with hydrofluoric acid. As HF selectively dissolves silicon dioxide, the elevations vanish after the treatment with minor effects on the order of the platinum nanoparticles. 


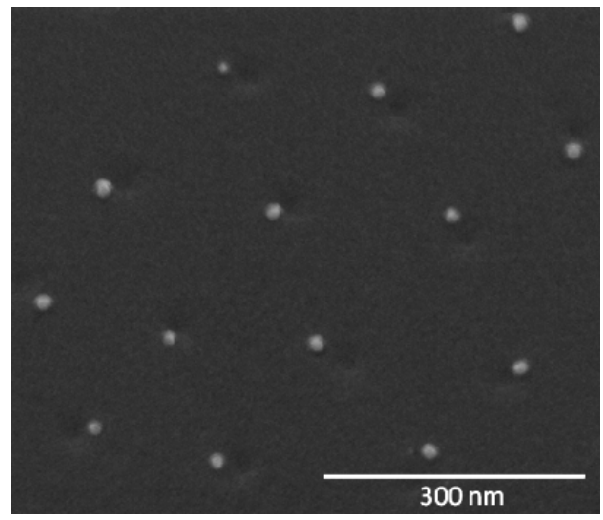

Figure 5.3.4. HF treatment of the platinum nanoparticle arrays. The circular elevations vanish after the treatment. Image provided by Achim Manzke; University of Ulm.

\section{Control over interparticle spacing}

In a second set of experiments, latex nanoparticles containing similar amounts of platinum but different sizes where prepared by a seeded emulsion polymerization process (chapter 5.1.2.5.7) and subjected to the etching process.

Table 5.3.2. Characteristics of latex particles with identical amounts of $\operatorname{Pt}(\mathrm{acac})_{2}$ but different diameters and the resulting nanoparticles arrays.

\begin{tabular}{|c|c|c|c|c|c|c|}
\hline \multirow{2}{*}{$\begin{array}{c}\text { Pt(acac) } \\
\text { added }\end{array}$} & \multicolumn{2}{|c|}{$\begin{array}{l}\text { Pt-content } \\
\text { measured }\end{array}$} & \multicolumn{2}{|c|}{ Final NP size } & \multirow{2}{*}{$\begin{array}{c}\text { Diameter } \\
\text { Latex } \\
\text { [nm] }\end{array}$} & \multirow{2}{*}{$\begin{array}{c}\text { Interparticle } \\
\text { Spacing } \\
\\
{[\mathrm{nm}]}\end{array}$} \\
\hline & [wt.-\%] & $\begin{array}{l}\text { [\% of } \\
\text { theory] }\end{array}$ & $\begin{array}{c}\text { theoretical } \\
\text { value* } \\
\text { [nm] }\end{array}$ & $\begin{array}{c}\text { measured } \\
\text { [nm] }\end{array}$ & & \\
\hline $4.0 * *$ & 1.26 & $75.5 * * *$ & 11 & $9 \pm 1$ & $132 \pm 6$ & $148 \pm 25$ \\
\hline--- & 0.76 & 101.3 & 11 & $9 \pm 1$ & $167 \pm 6$ & $182 \pm 28$ \\
\hline--- & 0.42 & 103.5 & 11 & $9 \pm 1$ & $193 \pm 6$ & $212 \pm 35$ \\
\hline--- & 0.24 & 99.1 & 11 & $9 \pm 1$ & $228 \pm 7$ & $235 \pm 41$ \\
\hline--- & 0.16 & 111.9 & 11 & $9 \pm 1$ & $254 \pm 10$ & $271 \pm 46$ \\
\hline
\end{tabular}

* calculated from the metal content of one colloid as determined by ICP-OES.

**the sample was used as seed for the subsequent increase in size

without adding further complex.

$* * *$ the amount of platinum in the seed particle was set to $100 \%$ for the determination of the platinum content of the seed particles.

Figure 5.3.5 presents the resulting nanoparticle arrays produced from colloids with a diameter between $132 \mathrm{~nm}$ and $254 \mathrm{~nm}$. In the first row, electron microscopy images are shown. A clear increase in interparticle spacing is visible from top (small colloids) to bottom (larger colloids). Histograms produced by a statistical examination of nanoparticle sizes and interparticle distances are shown beside the respective SEM images. Table 5.3.2 summarizes the characteristics of the different nanoparticle arrays. As expected, no change in the size of the 
individual platinum nanoparticles was detected; reflecting the identical amounts of platinum in the latex particles (compare chapter 5.1.2.5.7). The interparticle distance increases with increasing particle diameter. However, both spacing and standard deviation increase for the nanoparticle spacing compared to the particle diameter of the polymer colloids. This is attributed to defects present in the colloidal monolayer that are inherited by the nanoparticles and lead to larger distances between the individual particles when statistically analyzed.
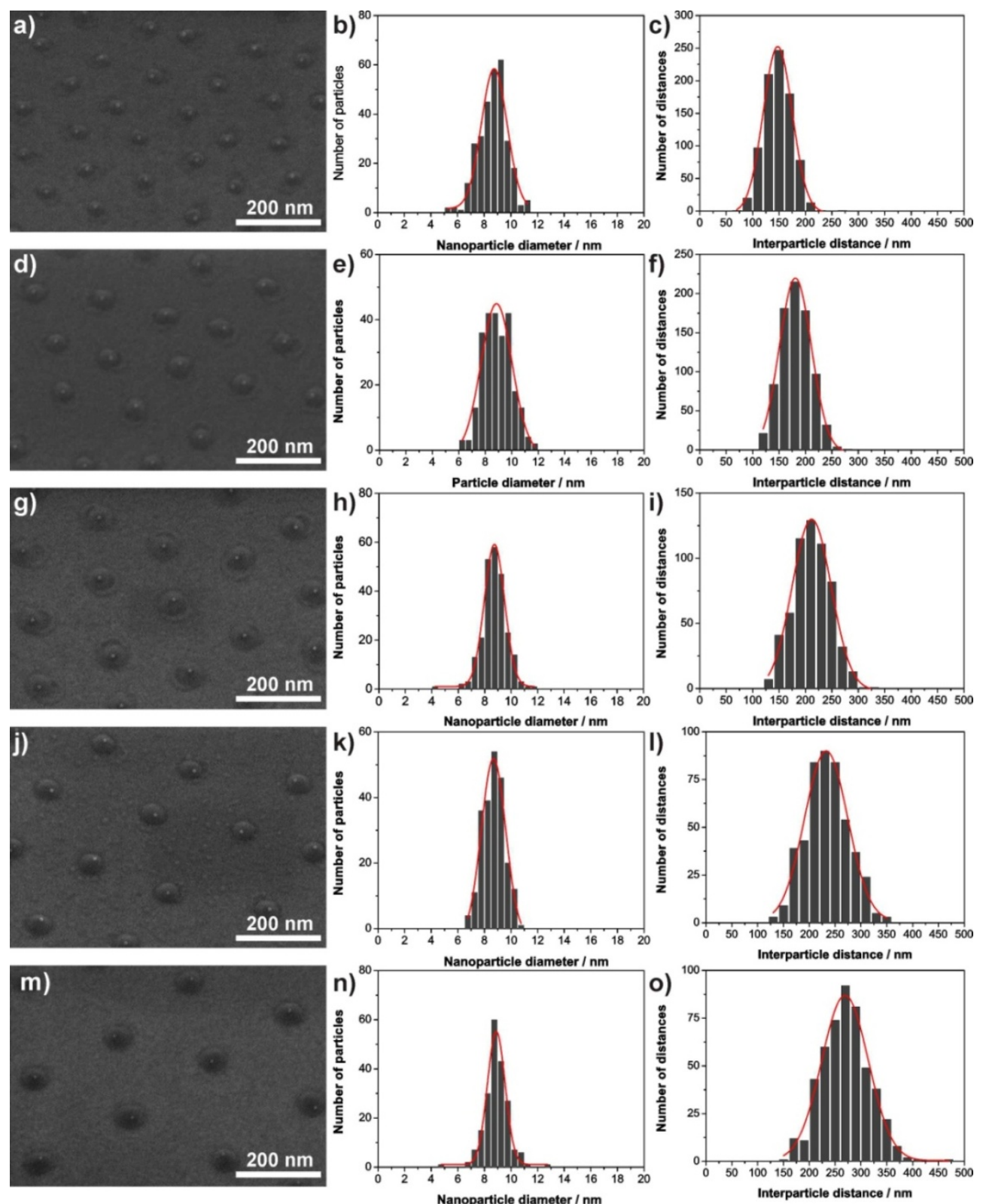

Figure 5.3.5 Control of interparticle distance in the non-conventional lithography process with platinum(II)acetylacetonate containing latex particles. All polymer particles contained a similar amount of $\mathrm{Pt}(\mathrm{acac})_{2}$ but different diameters. The first row shows electron micrographs of the arrays, the second and third row histograms of the particle size and interparticle distance respectively. a-c) $132 \mathrm{~nm}$ colloids; d-f) 167 nm colloids; g-i) 193 nm colloids; j-1) 228 nm colloids; m-o) 254 nm colloids. Experiments performed and images provided by Achim Manzke; University of Ulm. 


\subsubsection{FePt alloy nanoparticle arrays}

After the demonstration of a high degree of control in adjusting both particle size and interparticle distance, the approach was extended to the creation of more complex metallic materials. Iron-platinum alloy nanoparticles were chosen as target structures for two reasons. First, the compound is a complex alloy that only forms if the stoichiometry is precisely controlled. Therefore, it represents a significantly more challenging approach as compared to single compound particles. Second, the magnetic properties of the ferromagnetic $\mathrm{L} 1_{0}$ phase might be of interest for future applications in data storage.

The synthesis of latex particles containing both iron and platinum complexes and their characterization with respect to metal contents and stoichiometry of both elements was described in chapter 5.1.2.4.1.

Subsequently, the prepared latex dispersions were casted into a colloidal monolayer and treated by a similar etching procedure as the platinum containing latexes of the previous chapter by Achim Manzke at the University of Ulm. Oxygen plasma was applied for 25 min followed by thermal annealing at $650^{\circ} \mathrm{C}$ for $120 \mathrm{~min}$ in vacuum and at $1000{ }^{\circ} \mathrm{C}$ in oxygen atmosphere for 10 min. Figure 5.3.6 presents details of the preparation process from the assembly of the colloidal particles to the etched, inorganic nanoparticles.
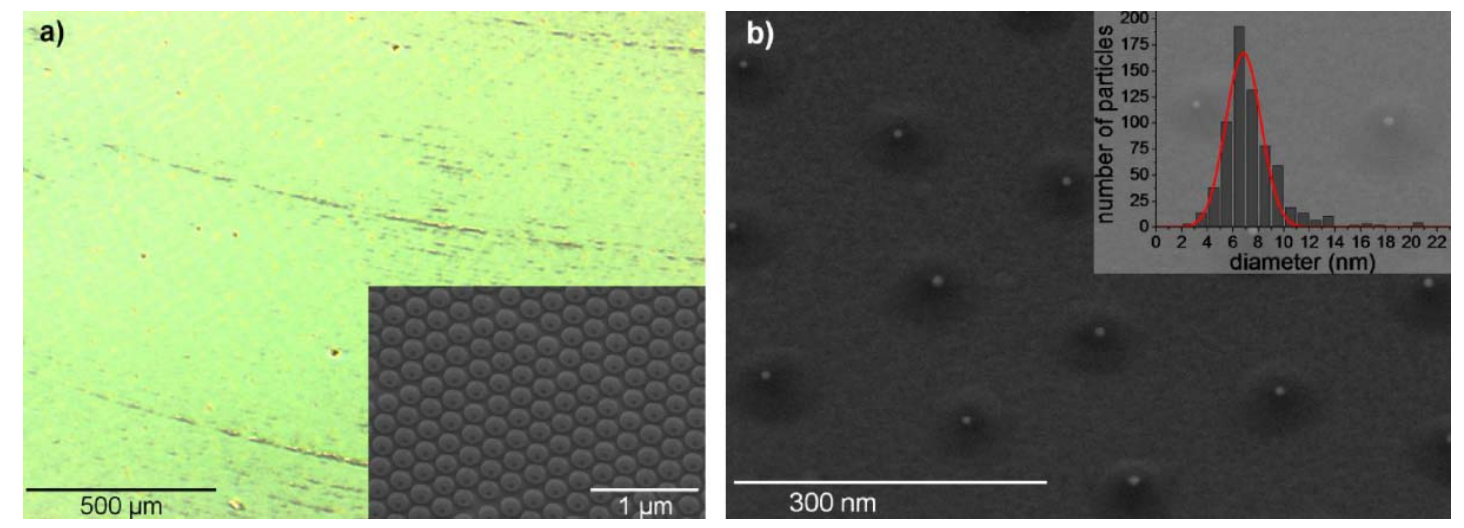

Figure 5.3.6. Monolayer of Fe- and Pt-precursor loaded PS spheres deposited by dip-coating onto hydrophilic $\mathrm{Si} / \mathrm{SiO} 2$ substrate over large scales as demonstrated by optical microscopy (a). The inset of

(a) presents a SEM image of slightly diameter-reduced PS particles. Continuous size reduction of PS colloids is accomplished by oxygen plasma treatment without disruption of their original hexagonal order. Subsequent annealing to $650^{\circ} \mathrm{C}$ in vacuum for $120 \mathrm{~min}$ and $1000^{\circ} \mathrm{C}$ in oxygen atmosphere for $10 \mathrm{~min}$ lead to corresponding hexagonally ordered FePt NP arrays (b). The lens-like shape of the local particle surroundings is discussed below (cf. Fig. 4 and text). The NPs exhibit spherical shape and Gaussian size distribution with average diameter of ca. $6.8 \pm 1.4 \mathrm{~nm}$ (inset of b). Experiments performed and images provided by Achim Manzke; University of Ulm. 
In the left part (a), an optical microscopy image of the monolayer is shown to highlight the uniform coating of the substrate with colloids. The inset shows an electron micrograph of the monolayer after one minute of plasma treatment and underlines the conservation of the colloid positions. Figure 5.3.6b shows the resulting metal nanoparticles after the thermal annealing procedure. The symmetry of the monolayer is retained and the metal nanoparticles sit on elevations attributed to silicon dioxide formed by oxidation of the wafer substrate as described above. The inset shows a particle size histogram and the average size of the particles is determined to be approximately $7 \mathrm{~nm}$.

A detailed characterization of the magnetic nanoparticle arrays was subsequently performed at the university of Ulm. However, the measurements are not subject of the thesis and are discussed in detail in the publication "Bimetallic alloy nanoparticles prepared by miniemulsions". To prove the existence of crystalline FePt alloy particles, a transmission electron microscopy (TEM) image, taken by the group of Prof. Ute Kaiser at the University of Ulm is shown in Figure 5.3.7. The upper part (a) shows a side-view image of the particles. The lentil-like elevation of silicon dioxide is well visible.

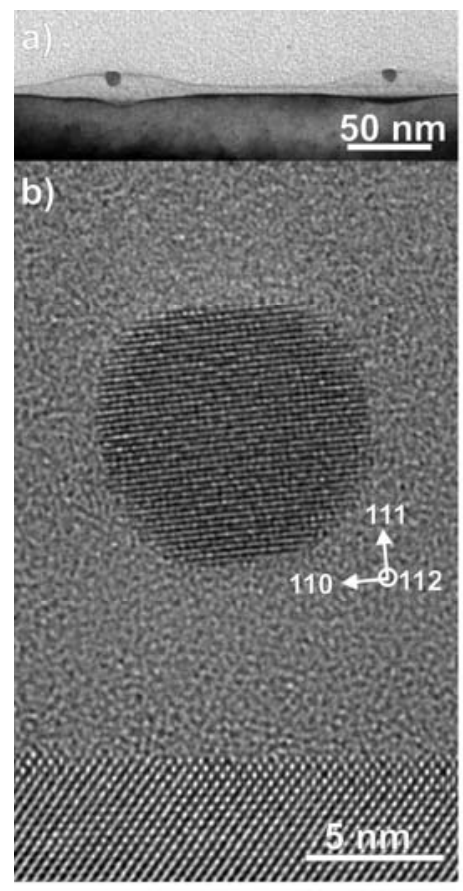

Figure 5.3.7. The top TEM image shows two FePt NPs embedded in a lens-shaped silicon oxide layer on a silicon substrate. Silicon oxide is growing faster under and around the particles compared to naked substrate due to the catalytic effect of Pt. The HRTEM image clearly demonstrates the crystalline structure of the FePt NPs. By analysis of in total 13 NPs an average lattice fringe spacing of $(0.2225 \pm 0.0058) \mathrm{nm}$ is determined as expected for (111) lattice planes of fcc FePt. Experiments performed and images provided by Ute Kaiser; University of Ulm. 
Figure 5.3.7b shows a high resolution TEM image of a single particle. The single-crystalline character of the particle can be seen by the individual lattices fringes. A comparison of the lattice spacing with values reported for the $\mathrm{L} 1_{0}$ phase of $\mathrm{FePt}$ in literature is in good agreement with the experimental results. A detailed discussion of the TEM images and the evaluation of the lattice spacing and crystal structures as well as a detailed characterization of the magnetic properties of the alloy particles can be found in the article mentioned above. 


\subsubsection{Plasmonic structures from non-close packed monolayers}

In this chapter, the non-close-packed monolayers produced as described in chapter 5.2.2 are used in a lithographic process to prepare crescent shaped gold nanostructures. These nanocrescents support multiple polarization dependent plasmon resonances. In close proximity, they undergo coupling processes (chapter 3.5.3) leading to hybrid resonances which are drastically shifted in energy with respect to the parental resonances of single crescents.

First, arrays of single gold nanocrescents are produced and the influences of the crescent dimensions and lattice spacing on the plasmonic properties of the material are discussed. Next, using more sophisticated approaches, two crescents are brought into close vicinity in order to observe plasmon hybridization of the individual crescent resonances. Two approaches are presented, leading to opposing and stacked double crescent architectures, respectively. In all cases, computer simulations were performed by Dr. Andreas Unger and Reza Mohammadi to support the models proposed. The double crescent structures were produced and analyzed in cooperation with Janina Fischer. The different results are published as separate manuscripts. The construction and optical properties of the crescent arrays are covered in "Electromagnetic Coupling in Two Dimensional Nano-Crescent Arrays". ${ }^{[238]}$ The stacked crescent dimers are published as "Plasmon Hybridization in Stacked Double Crescents Arrays Fabricated by Colloidal Lithography,"[212] and the opposing crescent architectures have been submitted as "Plasmon Hybridization and Strong Near-field Enhancements in Opposing Nanocrescent Dimers with Tunable Resonances". ${ }^{239]}$ 


\subsubsection{Arrays of gold nanocrescents}

\subsection{Introduction}

In nanoscale metallic objects, collective oscillations of electrons, known as plasmon resonances are excited upon interaction with electromagnetic irradiation. Their resonance wavelength crucially depends on size and geometry of the metallic structure. ${ }^{[240]}$ This phenomenon has been introduced in chapter 3.5 .

An assembly of such structures, featuring sizes below the wavelength of the incident radiation can be approximately described by an effective homogeneous refractive index, in analogy to the refractive index of ordinary matter which is based on the collective response of many individual molecules. ${ }^{[241]}$ These assemblies have been termed metamaterials as they can be designed to possess unique properties not occurring in nature, most prominently negative refraction. ${ }^{[242-243]}$ Split ring resonators have been found to be valuable building blocks for such materials. The incident light excites circulating currents in these structures, leading to local magnetic dipole moments that can counteract the incident magnetic field and result in negative permeability, a prerequisite for negative refraction. ${ }^{[24-246]}$

Since the size of the objects must be substantially smaller than the wavelength of the electromagnetic field, the fabrication of metamaterials at or close to optical wavelengths remain challenging. Major efforts have been undertaken to design periodic arrays of split ring resonators in 2 and 3 dimensions with high precision. Predominantly, modern lithographic techniques as electron beam lithography or focused ion beam milling are employed. Metamaterials in 2 and 3 dimensions have thus been produced ${ }^{[240,247-248]}$ and negative refraction has been demonstrated at various wavelengths approaching the visible range. ${ }^{[241,249-251]}$

These methods, priced for their resolution and positioning precision, however, intrinsically feature drawbacks that hamper widespread applications, both in larger scale technological applications as well as in academic research. First, expensive equipment is needed not being readily available at every facility. Second, due to their serial character, the production of arrayed structures, consisting of many individual objects is time consuming; large area patterning thus becomes cumbersome - if not impossible. Third, the quality of the noble metals - and with them the optical properties of the structures are in general compromised due to contaminations. These arise from residues of photoresist and amorphous carbon for electron beam processes as well as contamination- and doping with heavy ions employed by focused ion beam milling.

A conceptually simple, yet powerful alternative consists of the use of colloidal particles for the formation of nanostructures (chapter 3.4.1). Sacrificing the possibility to generate any desired 
object shape, it overcomes the major drawbacks of serial processing. Without the use of expensive instruments, large areas can be structured in a highly parallel fashion. Furthermore, as noble metals are deposited by vacuum evaporation, impurities and contaminations can be reduced to a negligible level.

As colloidal monolayers feature a high degree of symmetry and can be fabricated with particles of a broad range of sizes, precise control of size and lateral spacing of structures at the nanoscale is readily achieved. Arrays nanoscale crescents ${ }^{[136,252]}$ can be constructed using nonclose-packed monolayers, the preparation of which has been introduced in chapter 5.2.2. Such objects are of special interest as they support several multipolar resonances ${ }^{[129-130,148]}$ and can be described as nanoscale split ring resonators. Thus, a possibility to design metamaterials in a cost-efficient way is introduced.

This chapter introduces the preparation process for the arrays of split ring resonators. Theoretical calculations on the electromagnetic coupling in such arrays in dependence of the distance of the objects have been performed by Dr. Andreas Unger ${ }^{[238]}$ but are not scope of this thesis.

\subsection{Preparation of samples}

The preparation process for the nanocrescents arrays is schematically shown in Figure 5.3.8. A close-packed monolayer (a) is exposed to oxygen plasma to produce a non-close architecture (b). Gold with a thickness of $50 \mathrm{~nm}$ is evaporated with an angle of $30^{\circ}$ with respect to the surface normal as indicated by the arrow (b). Subsequently, a reactive ion beam etching (RIE) step is performed normal to the surface to remove the gold film at parts of the surface accessible by the beam. The areas shaded by the colloids remain unaffected (d). The colloid monolayer is removed mechanically using adhesive tape while the crescent structures remain on the surface (e). By variation of the size reduction of the polymer colloids, the size of the resulting crescents can be precisely adjusted. The lattice constant of the array is given by the initial diameter of the colloidal particles applied as mask. 


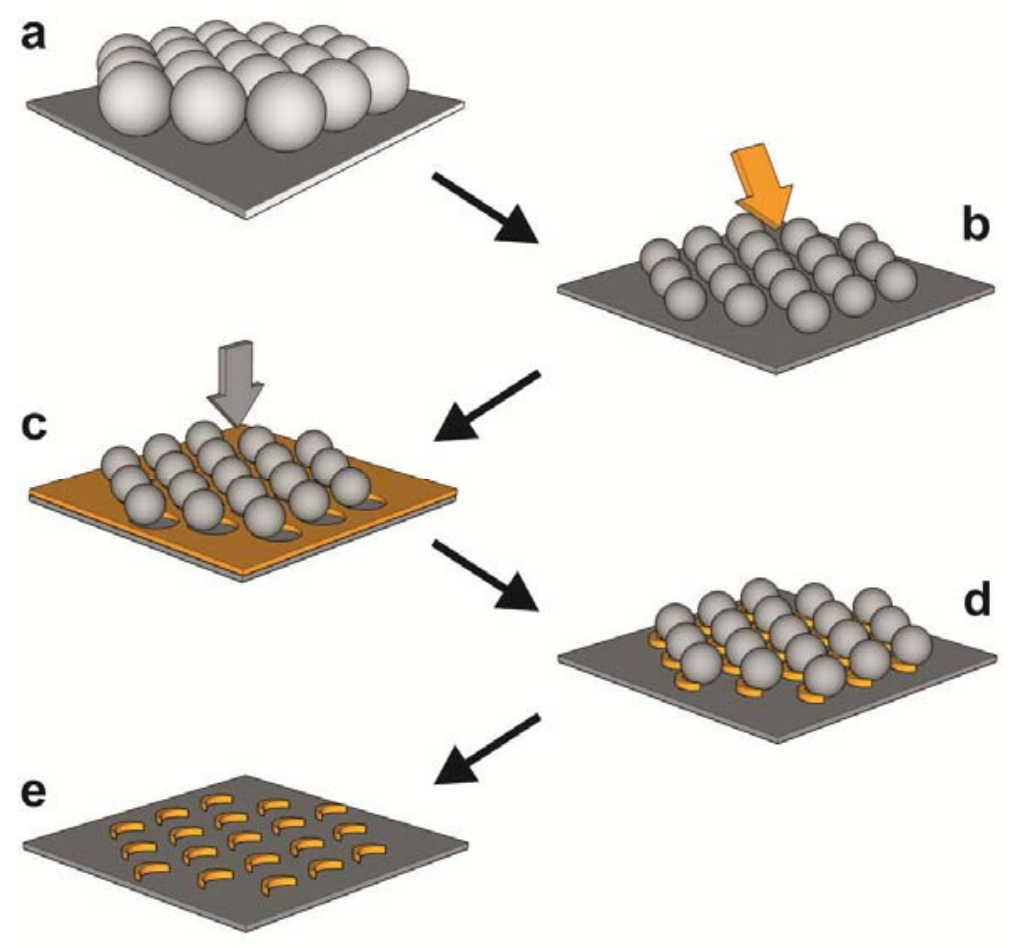

Figure 5.3.8. Fabrication of the nanostructure arrays: (a) monolayer formation, (b) size reduction and metal deposition, (c,d) ion etching, (e) mask removal.

\subsection{Characterization of the crescent arrays}

Arrays of crescents with similar lattice spacing and different sizes were produced from colloidal monolayers with an initial diameter of $408 \mathrm{~nm}$. The plasmonic properties of these crescent arrays were analyzed using polarization dependent UV/Vis-NIR spectroscopy. Figure 5.3.9 presents electron micrographs and optical spectra of the produced samples.

The size of the crescents was varied between $266 \mathrm{~nm}$ and $153 \mathrm{~nm}$ by variation of the plasma treatment time of the colloids. The high degree of order in the arrays that exhibit the hexagonal arrangement of the colloidal monolayer is clearly visible. While the crescents' contour is very regular for the first two samples $(266 \mathrm{~nm}$ and $248 \mathrm{~nm})$, the smaller crescents $(193 \mathrm{~nm}$ and $153 \mathrm{~nm}$ ) are increasingly roughened and strong deviations from the typical crescent shape appear for the smallest crescents. This is a result of the increasing surface roughness of the colloids in the course of the etching procedure. 

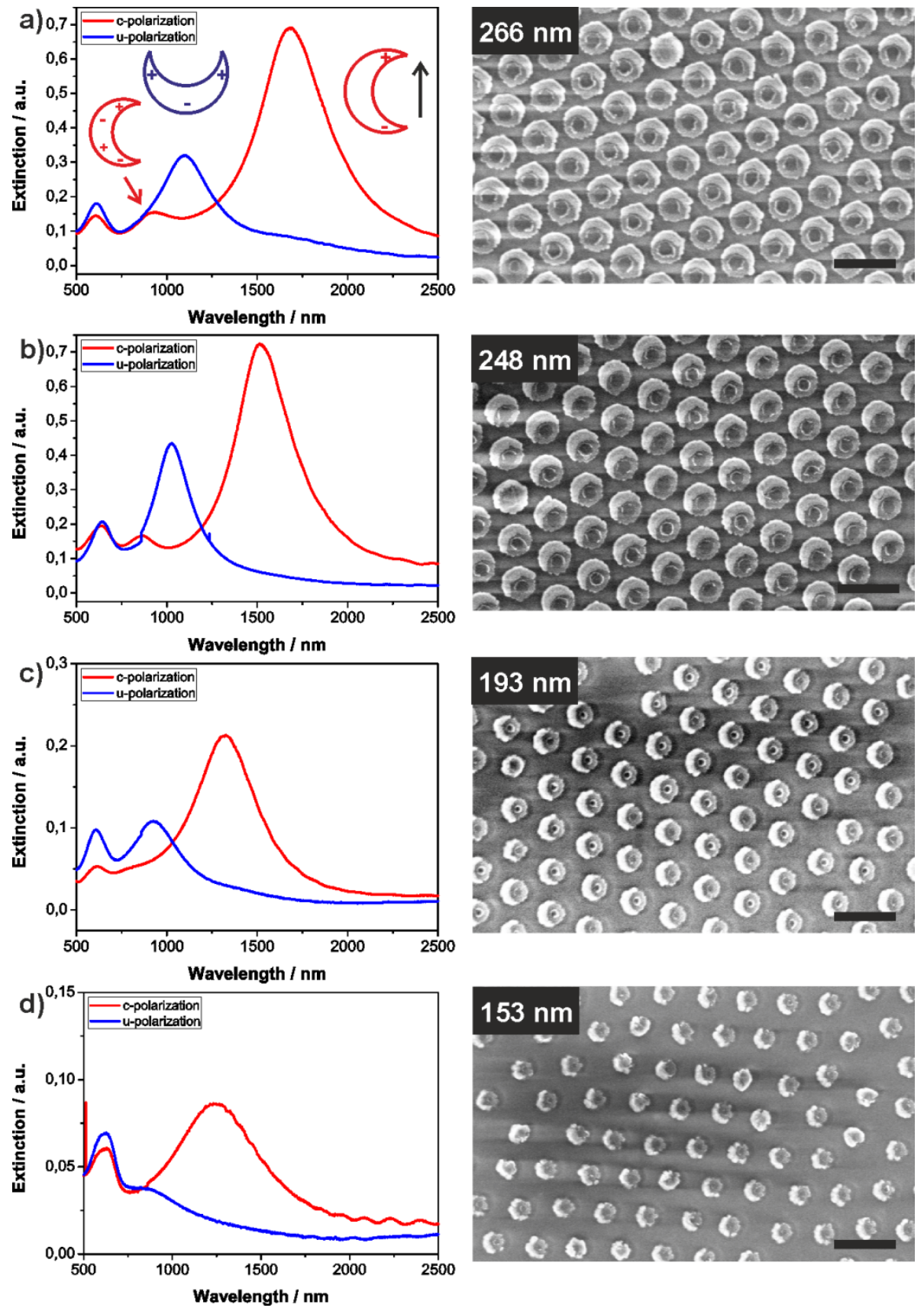

Figure 5.3.9. Optical investigation of crescent arrays with different nanostructure size but similar lattice constant. Left row: UV/Vis-NIR spectra. Schematic representations of the resonances excited in c- and upolarization are shown as inset in the uppermost spectrum. The black arrow indicates the polarization of the electric field with respect to the crescent structure. Right row: SEM micrographs of the corresponding arrays. The mean tip-to-tip distance of the crescents is shown in the images.

The optical properties of the crescent arrays depend on the polarization of the incident light.

In general, two characteristic resonances can be excited. A sketch of the charge distribution of the two basic resonances is shown in Figure 5.3.9a. The fundamental plasmonic mode, termed $\mathrm{c}_{1}{ }^{[130]}$ resonance is excited when the electric field vector is parallel to the long axis of the crescent. The classification as "c"-resonance is given as the crescent appears as the letter $\mathrm{c}$ when 
the electric field vector is assumed to be vertical. It features one node in the middle of the crescent and opposing charges at the crescent tips (Figure 5.3.9a, red crescent). For the sample with $266 \mathrm{~nm}$ crescents, this resonance appears at $1692 \mathrm{~nm}$. The first harmonic, coined $\mathrm{u}_{1}{ }^{[130]}$ (as the crescent appears u-shaped with the electric field vector being vertical) is excited only with a polarization of light rotated $90^{\circ}$ with respect to the $\mathrm{c}$ resonance case. The charge distribution, represented in blue in Figure 5.3.9a, shows similar phases in the tips, an opposing maximum at the center of the crescents and two nodes located in the wings of the crescents. For the first array presented in Figure 5.3.9a, the $\mathrm{u}_{1}$ resonance is excited at $1098 \mathrm{~nm}$. A second resonance, c2, can be excited at higher energies. ${ }^{[130]}$ It can be described as the second harmonic, featuring two nodes and a charge distribution as shown in the inset of Figure 5.3.9a. For the array of $266 \mathrm{~nm}$ crescents, the c2 resonance appears at $925 \mathrm{~nm}$. Finally, a polarization-independent resonance is excited at even smaller wavelengths. This resonance was named particle plasmon (pp) resonance ${ }^{[130]}$ and corresponds to a resonance excited across the short axis of the crescent.

The decrease in size of the crescents is reflected by a blue shift of all polarization dependent resonances. Only the particle plasmon resonance is not affected by the size of the crescent. The resonance positions of the different crescent arrays are summarized in Table 5.3.3.

The increased roughening of the smaller colloids is reflected by a broadening of the plasmon resonances. Another consequence of the roughening is that the second harmonic $\mathrm{c} 2$ resonance is not resolved in the spectra of the smallest particle arrays (Figure 5.3.9c and d).

Table 5.3.3.Mean crescent size and resonance position of the crescent arrays shown in Figure 5.3.9.

\begin{tabular}{ccccc}
\hline $\begin{array}{c}\text { Crescent size } \\
\text { (tip to tip) } \\
\text { [nm] }\end{array}$ & Lattice spacing & \multicolumn{3}{c}{ Resonance position } \\
& [nm] & $\begin{array}{c}\text { c-resonance } \\
\text { [nm] }\end{array}$ & $\begin{array}{c}\text { u-resonance } \\
\text { [nm] }\end{array}$ & $\begin{array}{c}\text { particle resonance } \\
\text { [nm] }\end{array}$ \\
\hline $266 \pm 9$ & 408 & $1682 ; 925$ & 1098 & 610 \\
$248 \pm 9$ & 408 & $1516 ; 863$ & 1028 & 621 \\
$193 \pm 10$ & 408 & 1323 & 927 & 611 \\
$153 \pm 10$ & 408 & 1248 & 858 & 614 \\
\hline
\end{tabular}

In summary, the experiments clearly demonstrate that the size of the individual crescents can be controlled independent of the lattice constant of the array. This can be used to tailor the resonance positions with possible applications for the design of metamaterials. Using computer simulations, the effect of interparticle coupling in such arrays in dependence of the distance of the individual crescents has been investigated and compared to the arrays constructed 
experimentally. ${ }^{[238]}$ It has been found that two distinct coupling regimes (weak and strong coupling) can be realized.

\subsubsection{Stacked double crescent arrays}

\subsection{Introduction}

Recently, 3D arrays of nano crescents have been fabricated by Retsch et al. in a layer-by-layer fashion using colloidal lithography. ${ }^{[136]}$ The approach uses a sol-gel process to cover one layer of nanostructures with insulating material followed by another lithography step. Thus, the mutual orientation of the crescents can be controlled. However, vertical alignment of the crescents with respect to the underlying layer has not yet been achieved using a colloidal approach for structuring.

The vertical alignment, paired with short separation distances introduces intriguing changes in the optical spectra of nanostructures arising from interaction of the plasmonic resonances. A theoretical model, termed plasmon hybridization, has been established to explain such coupling effects for nanoparticle dimers (chapter 3.5.3). ${ }^{[151-152]}$ This hybridization model can be seen as an electromagnetic analogue to molecular orbital theory: the plasmonic resonances of two nanostructures in close proximity to each other undergo a coupling process that gives rise to new resonances as linear superpositions of the original resonances. In this picture, the two particles can be described as a plasmonic molecule, ${ }^{[253-254]}$ similar to two atoms chemically bound to form a molecule. As the wave function of these atoms can be superimposed to give rise to a binding- and an antibinding molecular orbital in the molecule, the plasmonic modes hybridize as well. Recently, the concept has been extended to describe the coupling of the fundamental mode of split ring resonators. ${ }^{[248,254]}$

In this chapter, a quasi 3D fabrication technique leading to stacked nano-crescents arrays with precise vertical alignment using a colloidal lithographic approach is presented. The process leaves ample degrees of freedom to control various aspects of the structures, including size, lateral spacing, vertical spacing as well as mutual orientation, thus allowing for precise adjustment of the plasmonic resonances of the particle array.

Extended 3D structure generation as demonstrated by Retsch et al. ${ }^{[136]}$ is sacrificed in favor of precise control over the vertical alignment of the crescents and, thus, to gain access to the observation of strong coupling effects in the stacked crescents. 


\subsection{Results and discussion}

\section{Sample preparation}

Large areas of highly ordered stacked crescents arrays were produced by a colloidal lithography approach. A non-close-packed monolayer (Figure 5.3.10a) was used as a mask for the patterning process. Chromium (thickness: $1.5 \mathrm{~nm}$ ), used as adhesion promoter, and gold (thickness: $25 \mathrm{~nm}$ ) were evaporated under an angle of $30^{\circ}$ with respect to the surface normal (Figure 5.3.10b). Afterwards, the sample was rotated by $180^{\circ}$ and silicon dioxide (thickness: $20 \mathrm{~nm}$ ), chromium (thickness: $1.5 \mathrm{~nm}$ ) and gold (thickness: $25 \mathrm{~nm}$ ) were evaporated under an angle of $-30^{\circ}$. In this way, material was deposited in the areas under the colloids shaded by the colloidal spheres in the previous evaporation step (Figure 5.3.10c). Reactive ion beam etching (RIE) perpendicular to the surface was used to remove all material not protected by the colloids. Material deposited under the colloids was not affected by the ion beam and therefore, remained on the surface (Figure 5.3.10d). The colloidal monolayer was removed without inducing damage to the nanostructure array (Figure 5.3.10e). The process led to the formation of arrays of stacked double crescents.

The individual double crescents are separated by a thin layer of insulating silicon dioxide and hence, represent two individual plasmonic structures in very close proximity. Due to the $180^{\circ}$ rotation in between the two evaporation processes, the two crescents were symmetric with their tips facing each other and overlapping over a length of approximately $40 \mathrm{~nm}$. The 3D arrangement with one nano-crescent being on top of the other is visualized in Figure 5.3.10f. The diameter of the individual crescents was $450 \mathrm{~nm}$. The lattice spacing in the array of the crescents is determined by the colloidal monolayer as well and reflects its symmetry. For the actual structure array in this study, the interparticle spacing was approximately $100 \mathrm{~nm}$. 


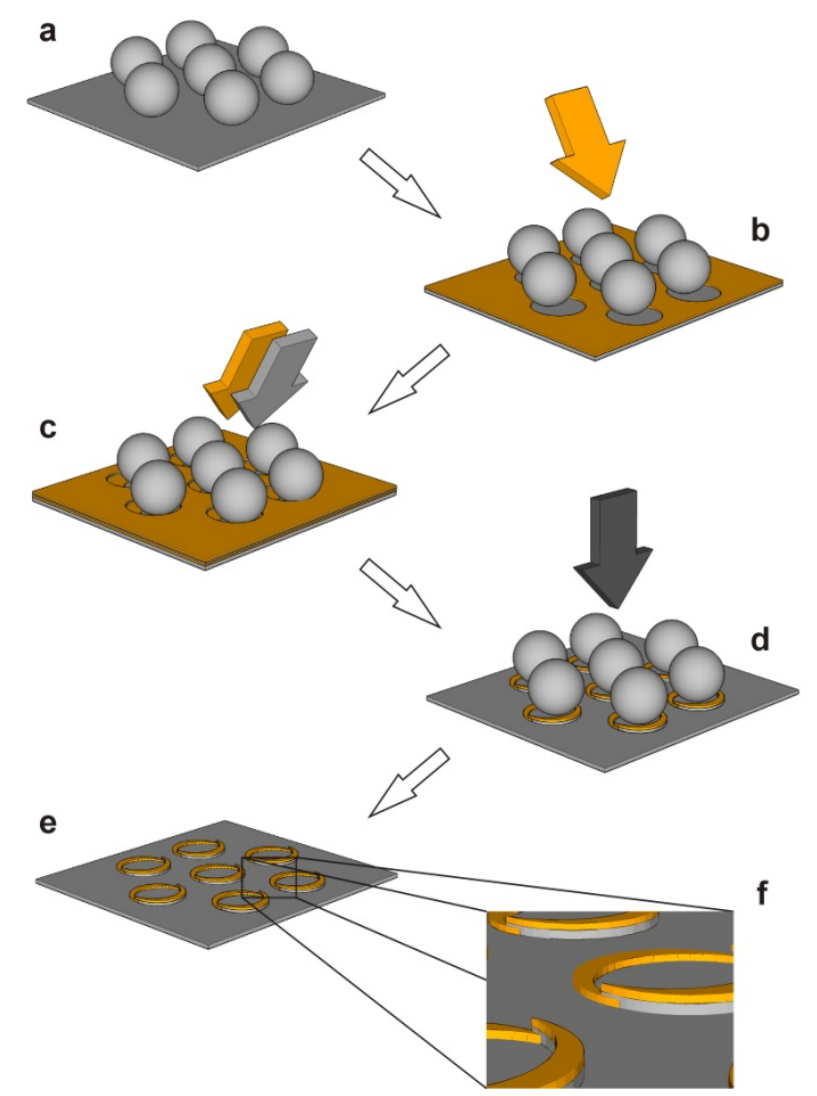

Figure 5.3.10. Process flow for the fabrication of stacked nano-crescent arrays. a) Assembly of the colloidal monolayer and subsequent plasma induced size reduction. b) Evaporation of gold under an angle of $30^{\circ}$ with respect to the surface normal. c) Evaporation of silicon dioxide and gold under an angle of $30^{\circ}$ with respect to the surface normal. d) Reactive Ion Beam Etching (RIE) normal to the surface to remove gold and $\mathrm{SiO}_{2}$ from the areas not protected by the colloids. e) Removal of the colloids to uncover the stacked crescent arrays. f) Close up of the crescent arrays to highlight the separation of the two crescents by the intermediate $\mathrm{SiO}_{2}$ layer.

Figure 5.3.11 shows SEM images of the prepared structures. To investigate the process at different stages and to produce reference samples with single crescents arrays, reactive ion etching was performed after the first evaporation of gold (upper part of Figure 5.3.11). This leads to the formation of single gold crescents in the areas protected by the colloids (Figure 5.3.11b). The complete, stacked double crescents structures are shown in Figure 5.3.11c and d. The left side of the Figure (a and c) presents top view images of the arrays of single (a) and stacked double crescents (c). In the top insets, the high symmetry of the arrays is well visible. The slightly undefined edges of the structures result from the fact that during the plasma etching process, the surface of the colloids roughened. This roughness is transferred to the nanostructures during the evaporation process. Side view images were taken before removal of the colloids to visualize the fabrication process and to investigate the vertical structure of the nanostructures. The emergence of the crescent structures in the regions shaded by the colloids is 
visible in Figure 5.3.11b. The side view image of the stacked double crescents (Figure 5.3.11d) clearly shows the separation of the individual single crescents by the silicon dioxide layer (indicated by arrows).

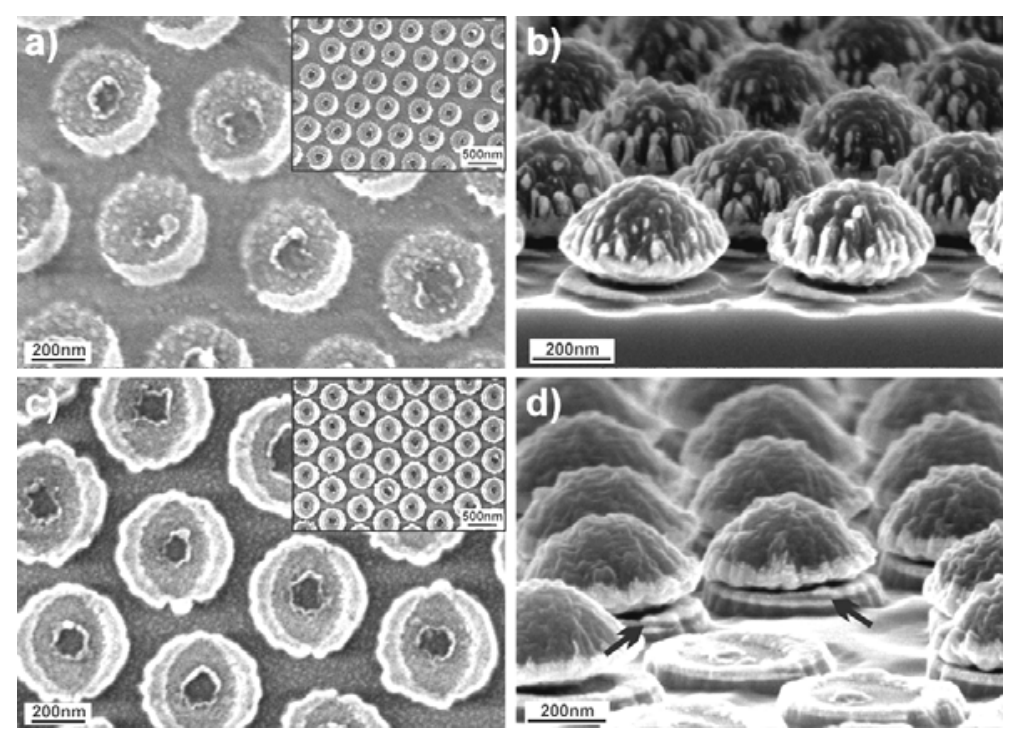

Figure 5.3.11. Scanning electron microscopy (SEM) images of the stacked nano-crescents arrays. Top row: single crescents arrays representing the first layer in the stacked crescent configuration. a) top view; b) side view. Bottom row: stacked crescents arrays. c) top view; d) side view. The black arrows highlight the separation layer between the two crescents. For a better illustration of the preparation method, the side-view images were taken on a sample where the colloidal monolayer used as mask was not removed.

The process leaves ample degrees of freedom to influence the resulting structures. Simplified sketches of these parameters are presented in Figure 5.3.12 and linked to the distinct parts of the construction process shown in Figure 5.3.10. Both, the size of the nanostructures and the lattice spacing in the array can be precisely adjusted by choosing colloids with different diameters and varying the etching time used to reduce the size of the particles (Figure 5.3.12a). Variation of the height of the silicon dioxide layer separating the stacked crescents enables the fine-tuning of the coupling efficiency of the two separate resonators. Another parameter to tune the structural design is the evaporation angle of gold that can be used to vary the overlap of the stacked double crescents (Figure 5.3.12b and c). Finally, changing the azimuthal angle for the second gold evaporation relative to the first one gives rise to completely different symmetries in the architectures of the stacked crescents as the second crescent can be oriented with any chosen angle with respect to the first one (Figure 5.3.12d). An azimuthal angle of $180^{\circ}$ as presented here leads to structures with a strong overlap on the edges with optical phenomena described below. Furthermore, the high symmetry of the stacked structures gives rise to resonances without a dipole moment, commonly referred to as dark modes. Such resonances are of importance for the creation of negative refraction materials as they can possess negative values for the permeability. [244-246, 248, 255] 

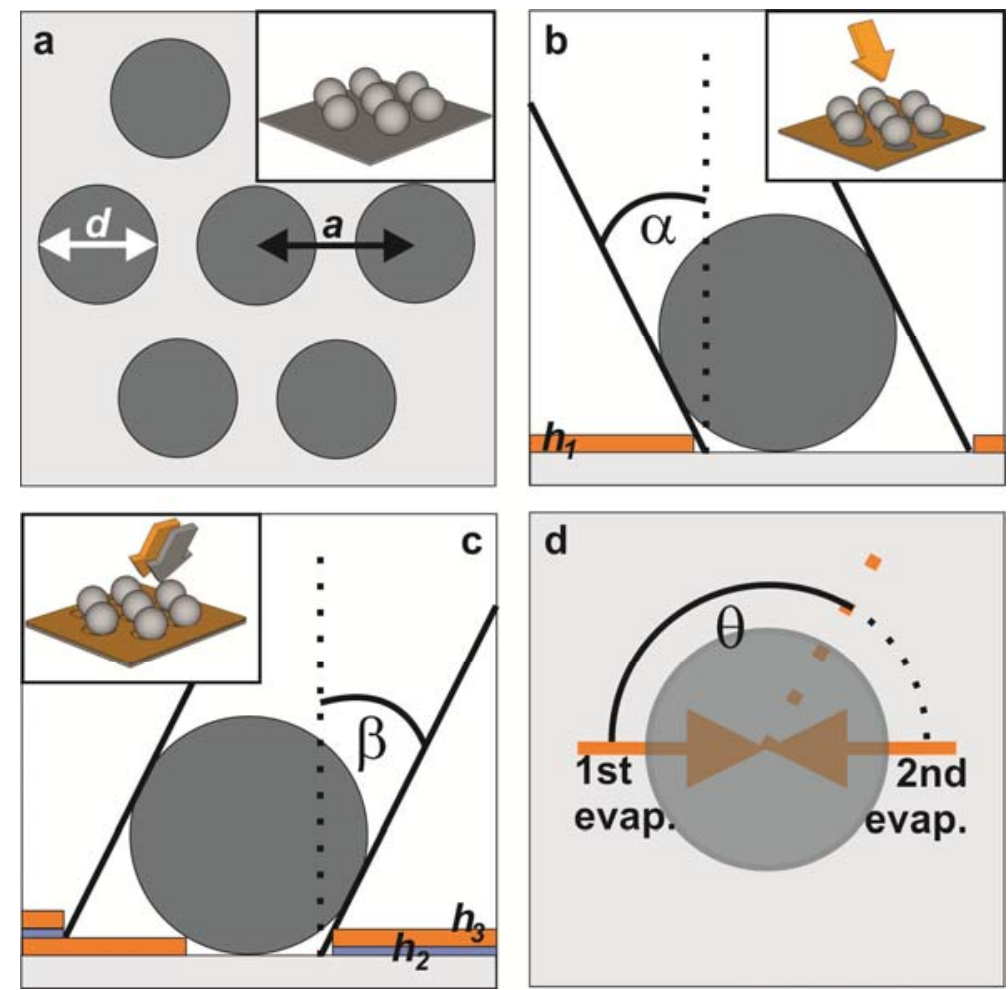

Figure 5.3.12. Detailed sketches of the fabrication process to define all important parameters that can be used to tailor the resulting structures. The insets link the sketches to various steps in Figure 5.3.10 of the main text. Dimensions are not to scale. 1) Top view of the non-close-packed monolayer. 2) Side view of the first evaporation step to deposit gold with an angle $\alpha$ with respect to the surface normal. 3) Side view

of the second evaporation step to deposit the silicon dioxide spacer and the second gold layer with an angle $\beta$ with respect to the surface normal. 4) Top view of the substrate to introduce the azimuthal angle $\theta$ that defines the mutual orientation of the two crescents in the stacked crescent structure. For the structures created, an angle of $180^{\circ}$ was used. The dotted line indicates the possibility of varying this angle to create structures with different symmetries.

A drawback of the fabrication technique presented in this paper is the inherent restriction in $3 \mathrm{D}$ space for the structure generation. This stems from the fact that colloids are used for the crescents generation which provide limited space underneath to shade the structures to be formed during the reactive ion etching step (compare Figure 5.3.11d). Therefore, the stacking of multiple crescent structures will be difficult. However, choosing bigger colloids, the stacking of several layers of larger crescents is feasible due to their comparably low height (typically, the height of a single crescent was $25 \mathrm{~nm}$ ).

Sacrificing extended 3D structure design, access is gained to perfect alignment of the nanostructures, a feature that has not yet been accomplished with a colloidal lithography process. As demonstrated by samples prepared by electron beam lithography with all the limitations discussed above, the vertical alignment of the separate crescents makes them valuable as plasmonic molecules with strong interparticle coupling of the individual resonators plasmonic resonances. ${ }^{[248]}$ 


\section{Plasmonic properties of the stacked double crescent architectures}

UV/Vis-NIR spectroscopy was used to gain fundamental understanding of the optical properties of the stacked double crescent structures. Figure 5.3.13 presents the polarization dependent optical spectra along with electron microscopy images of the fabricated structures. As objects, single crescents (a), stacked double crescents (b) and rings (c) were investigated and compared. The latter were generated by a similar process as the double crescents but without the intermediate silicon dioxide layer. Additionally, the optical properties of separated objects were compared to the arrayed structures.
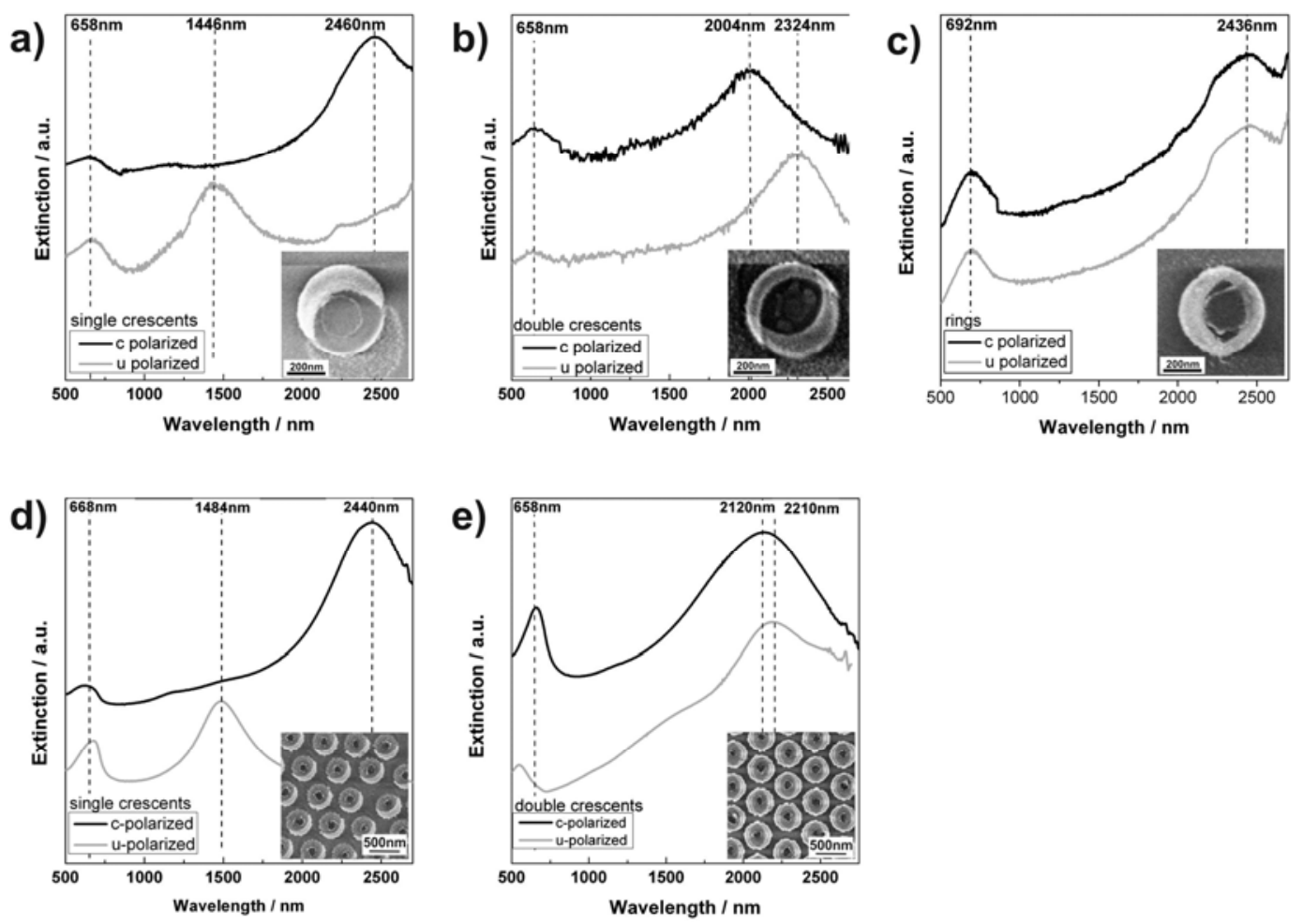

Figure 5.3.13. Polarization dependent UV/Vis-NIR investigation of plasmonic nanostructures. Individual objects are shown in a-c); arrays in d,e). a,d) single crescents; b,e) stacked double crescents; c) rings. SEM micrographs of the objects are shown as insets.

The objects show multiple plasmonic resonances that, in most cases, are highly sensitive to the orientation of the crescents relative to polarization of the incident light beam. As introduced in chapter 5.3.2.1.3, linear polarization along the symmetry plane of a crescent will be referred to as " $\mathrm{u}$ " polarization, polarization perpendicular to the symmetry plane will be termed "c" polarization. $^{[130]}$

Figure 5.3.13a shows the characteristic resonances of spatially separated, single crescents. In c polarization, a strong resonance at $2460 \mathrm{~nm}$ is visible that is attributed to the fundamental 
plasmon mode (termed $\mathrm{c}_{1}$ ) featuring one node in the middle of the crescent (see Figure 5.3.9a). ${ }^{[129-130,148]}$ In u polarization, the $c_{1}$ resonance disappears and a resonance at $1446 \mathrm{~nm}$ is excited. This resonance, termed $\mathrm{u}_{1}$, is attributed to the first harmonic (see Figure 5.3.9a). Another peak at approximately $658 \mathrm{~nm}$ is visible in both spectra. This peak, previously termed pp (particle plasmon) as it roughly matches the resonance observed for very small gold objects, is assigned to a polarization of the crescents perpendicular to the contour. ${ }^{[130,148]}$ For connected crescent structures forming a closed ring, two resonances are excited at $2436 \mathrm{~nm}$ and $692 \mathrm{~nm}$, none of which shows any dependence on the polarization of the incident light (Figure 5.3.13c). This behavior reflects the symmetry of a ring which does not show any anisotropy and thus, no polarization dependence. The low energy peak at $2436 \mathrm{~nm}$ is assigned to the fundamental ring resonance that is comparable in energy to the fundamental resonance of the single crescents. ${ }^{[130]}$ The high energy peak arises from the particle plasmon resonance excited perpendicular to the ring contour.

Spatially separating the two crescents to the stacked structure (Figure 5.3.13b) has drastic influences on the plasmonic resonances. It is obvious that the spectra differ strongly from the ones for rings, indicating that the thin separation layer has a dramatic influence on the optical properties. In agreement with the electron microscopy characterization (Figure 5.3.11), it becomes clear that the stacked crescent structures are indeed not ring-like.

For c-polarized incident light, a peak emerges at $2004 \mathrm{~nm}$ that is blue-shifted compared to the single crescents. $\mathrm{U}$ polarization gives rise to a resonance at $2324 \mathrm{~nm}$ being strongly red-shifted with respect to the single crescents. The fundamental resonance $c_{1}$ for single crescents is energetically lower compared to the first harmonic $\left(\mathrm{u}_{1}\right)$ appearing in $\mathrm{u}$ polarization, in accordance to the number of nodes of the standing wave excited in the crescent (see single crescents in Figure 5.3.14a and d). The introduction of a second crescent in close proximity, however, leads to a resonance visible in u polarization with a lower energy compared to the plasmon mode excited in c polarization. In total, the energetic order of the resonances is reversed compared to the parent resonances $\mathrm{c}_{1}$ and $\mathrm{u}_{1}$ of the single crescents.

A high energy peak, being visible in both polarizations, is present at $658 \mathrm{~nm}$. The latter appears at the same wavelength as for the single crescents and similarly, is attributed to the resonance perpendicular to the contour of the crescents.

To explain the striking differences in the optical properties, a plasmon hybridization model was adopted to the stacked structures. ${ }^{[151-152,253-254]}$ 

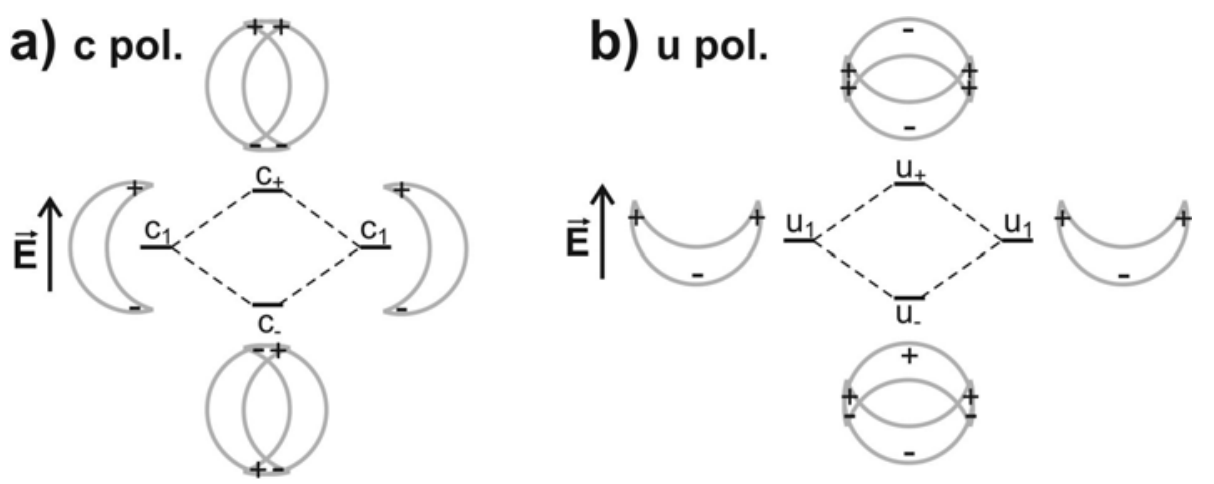

Figure 5.3.14. Plasmon hybridization model used to explain the shifts of the plasmonic resonances in the double crescent structures: a) linear superposition of two single crescents in c resonance: the close proximity results in a new coupled pair consisting of symmetric mode $\mathrm{c}_{+}$and an antisymmetric mode $\mathrm{c}_{-}$ with c. being energetically lower compared to $c_{+}$due to the off-phase oscillation that leads to charges with opposite sign to be in direct vicinity. b) Coupling scheme for resonances excited upon irradiation with $u$ polarized light: Similar to e), the close proximity of the two crescents lead to hybridization of the single plasmon resonances that split up into the symmetric mode $u_{+}$and the antisymmetric mode $u_{\text {.. }}$ In analogy to e), the antisymmetric mode $u_{-}$is lower in energy.

Figure 5.3.14 shows the formation of hybridized plasmon modes both for the $\mathrm{c}_{1}$ and $\mathrm{u}_{1}$ resonance of the single crescent. In both cases, the linear superposition leads to a pair of new modes. In the symmetric mode, termed $c_{+}$and $u_{+}$respectively, the two crescents oscillate in phase, whereas in the antisymmetric hybrid mode (c. and u.), the oscillation occurs out of phase. The antisymmetric mode is reduced in energy compared to the symmetric one.

For c polarized light, the symmetric mode $\mathrm{c}_{+}$features an enhanced dipole moment compared to the single crescents as the two crescents oscillate in phase (Figure 5.3.14a). Hence, the coupling efficiency to the external electric field is high and the $c_{+}$mode is detected in the spectrum shown in Figure 5.3.13b and e. In contrast, the out of phase oscillation of the antisymmetric mode c. leads to an electric field distribution in the stacked structure with no effective dipole moment present. Therefore, this mode cannot be excited by the external field and does not appear in the spectra. ${ }^{[255]}$ In total, the plasmonic resonance visible in the spectra is blue shifted compared to the single crescents' fundamental mode $\mathrm{c}_{1}$.

For u-polarized light, the physical model is similar to the $\mathrm{c}$ resonance: linear superposition of

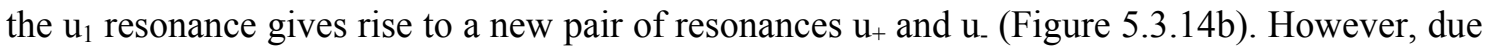
to the different symmetry of the $\mathrm{u}_{1}$ mode, the situation is inverted: The symmetric mode $\mathrm{u}_{+}$ shows an electric field distribution resembling an electric quadrupole without a dipole present in the plasmonic molecule. Hence, the coupling efficiency to the external light field is weak and the resonance does not appear in the spectrum of Figure 5.3.13b and e. The antisymmetric mode u. however, does feature an electric dipole and thus, is excited by u-polarized light. Compared to the single crescents' $u_{1}$ resonance, the hybrid resonance being visible in the spectrum is red shifted. 
The coupling efficiency between the two crescents is sufficiently strong to induce a cross-over of the plasmonic resonances compared to the single crescents with the lowest energy mode appearing in u-polarization. The given picture is in agreement with theoretical and experimental investigations of double split ring resonators produced by electron beam lithography, where plasmon hybridization was observed for the fundamental mode of the split rings. ${ }^{[248,254]}$

The spectra for separated objects and crescent arrays closely resemble each other (Figure 5.3.13a,b and d,e). In the particle arrays, all optical features are retained, including the crossover of resonances upon hybridization. Compared to the separated objects, the peaks of both hybridized plasmon modes in the stacked double crescents arrays are shifted by about $100 \mathrm{~nm}$ in the direction of the resonances for single crescents. This indicates a slightly decreased coupling efficiency which is probably caused by a small deviation in the height of the silicon dioxide spacer layer and by the increase in roughness of the arrayed structure.

The broadening of the peaks in the arrays is attributed to the increase in inhomogeneities. The higher surface coverage of the arrays is reflected by the increased extinction that was more than one order of magnitude higher than for the separated objects.

\section{Theoretical investigations}

In order to gain further insight into the response of the nanostructures to an external optical field, computer simulations of the optical properties using the finite element method with the software package JCMwave ${ }^{[256] 252]}$ were performed by Reza Mohammadi. The dielectric function used to model Au was based on the Drude model and fitted to experimental data of Johnson and Christy. ${ }^{[257]}$ The single and double crescents' geometries were adapted from SEM images to match the experimental design.

First, single crescents were simulated and the resulting spectra are shown in Figure 5.3.15. In c polarization, three resonances are visible. The fundamental plasmon mode $c_{1}$ appears at $2176 \mathrm{~nm}$ and, thus, agrees with the value observed experimentally with a blue-shift of $200 \mathrm{~nm}$. Additionally, the second harmonic resonance $c_{2}$ appears at $960 \mathrm{~nm}$. This resonance is not resolved in the experimental spectra, most probably due to insufficient intensity caused by a higher sensitivity of this mode to inhomogeneities in the nanoparticles structures. Finally, the mode assigned to the particle plasmon resonance appears at approximately $550 \mathrm{~nm}$. In $\mathrm{u}$ polarization, the first harmonic mode $\mathrm{u}_{1}$ becomes visible while the other modes are not excited, being in perfect agreement with both experimental data and models proposed. The mode peaks at $1265 \mathrm{~nm}$. Compared to the experimental spectra, the peak shows a blue-shift of $200 \mathrm{~nm}$. 


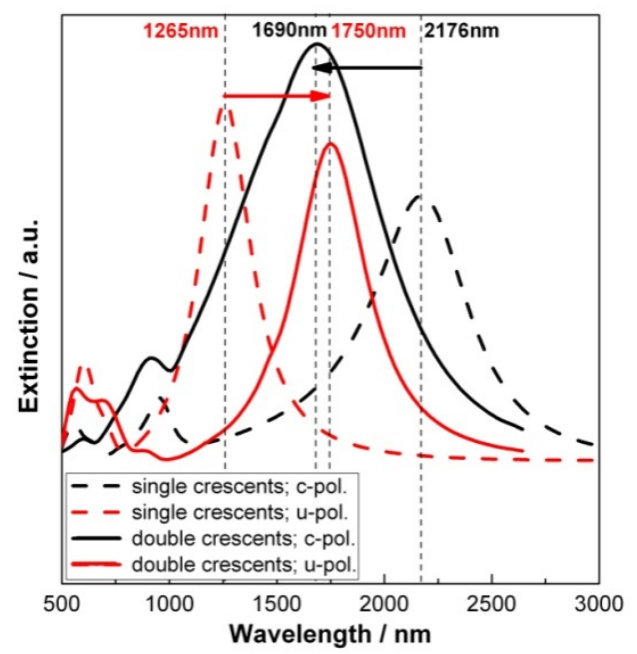

Figure 5.3.15. Simulated UV/Vis-NIR absorption spectra for single- and stacked double crescents for $\mathrm{c}$ and u polarization. The simulations reproduce the features of the experimental data and support the hybridization model.

The shifts of the resonances of the stacked double crescents with respect to the single crescents are in good agreement with the experimental results and support the plasmon hybridization model. For c polarized incident light, the hybrid resonance $\mathrm{c}_{+}$is strongly blue-shifted compared to the $\mathrm{c}_{1}$ resonance of the single crescents. Thus, it corroborates with the plasmon hybridization model, which predicts the only visible resonance to be the higher energy mode $c_{+}$. The peak appears at $1690 \mathrm{~nm}$, a value that is approximately $300 \mathrm{~nm}$ blue-shifted compared to the experimental value.

The hybrid analogue to the $c_{2}$ resonance appears at $910 \mathrm{~nm}$ and is blue-shifted compared to the $c_{2}$ resonance of the single crescents. The hybridization behavior of this resonance will be discussed in more detail further below.

In u polarization, the simulated spectrum shows one hybrid resonance at $1750 \mathrm{~nm}$. Compared to the first harmonic resonance $\mathrm{u}_{1}$ of the single crescents, the hybrid resonance $\mathrm{u}_{\mathrm{L}}$ is red-shifted and qualitatively agrees with the experimental results and the hybridization model. Moreover, the computer simulations also feature the cross-over of resonances observed experimentally. This validates the finding that the coupling efficiency of the plasmon modes is strong enough to shift the lowest energy mode from being observed in $\mathrm{c}$ polarization to the perpendicular $\mathrm{u}$ polarization.

Quantitatively, the hybrid mode $u_{\text {. }}$ is blue-shifted compared to the measured data. In general, the quantitative discrepancies between simulation and experiments are attributed to the following differences. First, roughness effects have been shown to cause a significant shift of the plasmon resonances that readily accounts for the blue-shifts of $200-300 \mathrm{~nm}$ of the simulated peak 
positions ${ }^{[238]}$ as has been shown for gold nanodots. ${ }^{[258]}$ Second, the precise determination of size and geometry of the double crescents is difficult as the first crescent is partially hidden underneath the second one. Additionally, the layer thickness of both gold and insulating layer is not constant for the complete crescent but diminishes around the wings and tips (see Figure 5.3.11).In the simulations however, it was taken as constant. Alteration of simulation parameters (such as values for the gold permittivity) as well as adapting more sophisticated models for the crescents (as including of the non-constant gold and $\mathrm{SiO}_{2}$ layers) could improve the quantitative agreement between simulation and experiment. However, as all essential features of the experimental data are reproduced, no further refinements were performed.

\section{The behavior of the hybrid $c_{2}$ resonance}

a)

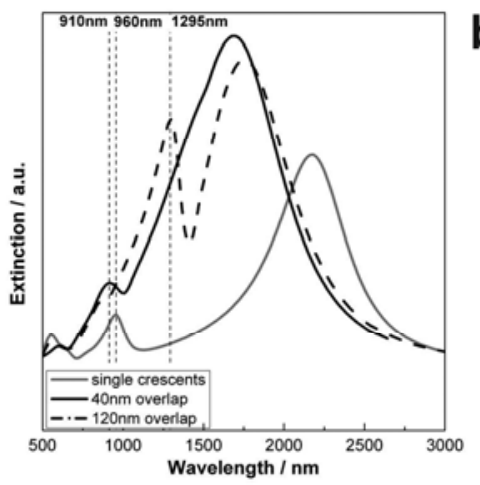

b)

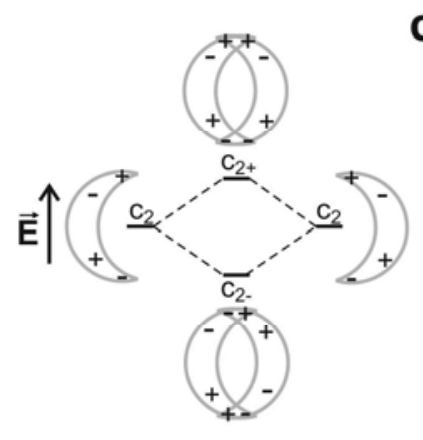

c)

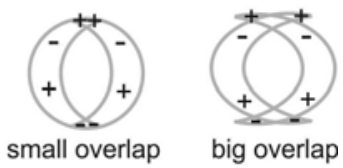

Figure 5.3.16. Hybrid plasmon mode arising from coupling of the $c_{2}$ resonance. a) Simulated spectra of stacked double crescents with varying overlap region. Compared to the single crescents' $c_{2}$ mode, the hybrid mode blue-shifts with small overlap $(40 \mathrm{~nm})$ while a strong red-shift is induced for the big overlap $(120 \mathrm{~nm})$. All $\mathrm{c}_{2+}$ resonance positions are indicated by a dotted line. b) Model of plasmon hybridization for the $c_{2}$ resonance. Linear superposition of the resonances lead to two hybrid modes termed $c_{2+}$ and $c_{2-}$ with $c_{2+}$ being higher in energy. c) Schematic representations of the charge distribution of the $c_{2+}$ mode for a small and a big overlap of the crescents. For the small overlap, the charges at the tips of the crescents are in close proximity. The mode is blue-shifted compared to the single resonance. With a big overlap, the tip charges cross the first node and come into closer proximity of the region with an opposing charge. Hence, the total energy of the hybrid mode is reduced.

The $\mathrm{c}_{2}$ resonance and its hybridized analogue were examined in further detail by means of simulations performed by Reza Mohammadi. According to literature, the resonance is the second harmonic mode and features two nodes and a charge distribution as illustrated in Figure 5.3.16b. ${ }^{[130,148]}$

Upon hybridization, the mode splits into the symmetric $c_{2+}$ and the antisymmetric $c_{2-}$ hybrid resonances (Figure 5.3.16b). In analogy to the hybridization of $\mathrm{c}_{1}$, only the symmetric $\mathrm{c}_{2+}$ mode features a sufficient dipole moment to be excited by the external field. Therefore, a blue-shift of 
the hybrid resonance compared to the single crescents $c_{2}$ resonance is expected and can be seen in the simulations of Figure 5.3.15.

Strikingly, the hybrid $c_{2+}$ mode shows a strong dependency on the overlap in the stacked dimer architecture while $c_{1}$ and $\mathrm{u}_{1}$ are not affected dramatically. The overlap describes the tip areas that directly face each other in the stacked structure. Experimentally, it can be increased by increasing the evaporation angle of gold and silicon dioxide (compare Figure 5.3.12). Figure 5.3.16a shows the simulated resonance spectra for single crescents and stacked double crescents with $40 \mathrm{~nm}$ and $120 \mathrm{~nm}$ overlap region of the crescents tips, with the first one representing the data shown in Figure 5.3.15. Upon hybridization, the peak shifts from $960 \mathrm{~nm}$ to $910 \mathrm{~nm}$ for a small overlap region of $40 \mathrm{~nm}$. The increase of the crescents' overlap region to $120 \mathrm{~nm}$ drastically changes the energetic situation for the $c_{2+}$ resonance. A strong shift to longer wavelengths is induced and the hybrid mode now peaks at $1295 \mathrm{~nm}$. Remarkably, the differences in overlap cause a change in shift direction with respect to the single crescents resonances.

This behavior is attributed to regions with opposite charge in the crescents wings as illustrated in Figure 5.3.16c. For a small overlap region, only the charges at the tips of the crescents interact and lead to the high energy mode $c_{2+}$. With an increase in overlap, the charges present at the tips are getting into closer proximity to the opposite charges present in the wings of the crescents, leading to a substantial decrease in energy of the hybrid mode. Both the fundamental $\mathrm{c}_{1}$ resonance and the first harmonic $\mathrm{u}_{1}$ do not have additional charges in the wings and therefore, are not sensitive to changes of the overlap region (compare Figure 5.3.16a for $\mathrm{c}_{1}$ resonance).

\section{Near field distributions}

In order to examine the charge distributions of the crescent dimers for the different hybrid resonances excited, near field distributions were mapped at the resonance frequencies (Figure 5.3.17). The upper part of the figure gives the field distributions in the xy plane, parallel to the substrate. The cuts were taken in the middle of the insulating barriers. Thus, both crescents are in equal distance to the displayed plane. The field essentially probes the distribution of charges predicted by the hybridization model. The lower part of Figure 5.3.17 shows the near-field distribution along the gap between the interacting tips of the two crescents. The cut shows the $\mathrm{xz}$ plane, being perpendicular to the substrate surface, and cuts the crescents parallel to the symmetry axis along the tip region. The dotted line inserted into the upper images shows the plane of the cut. 

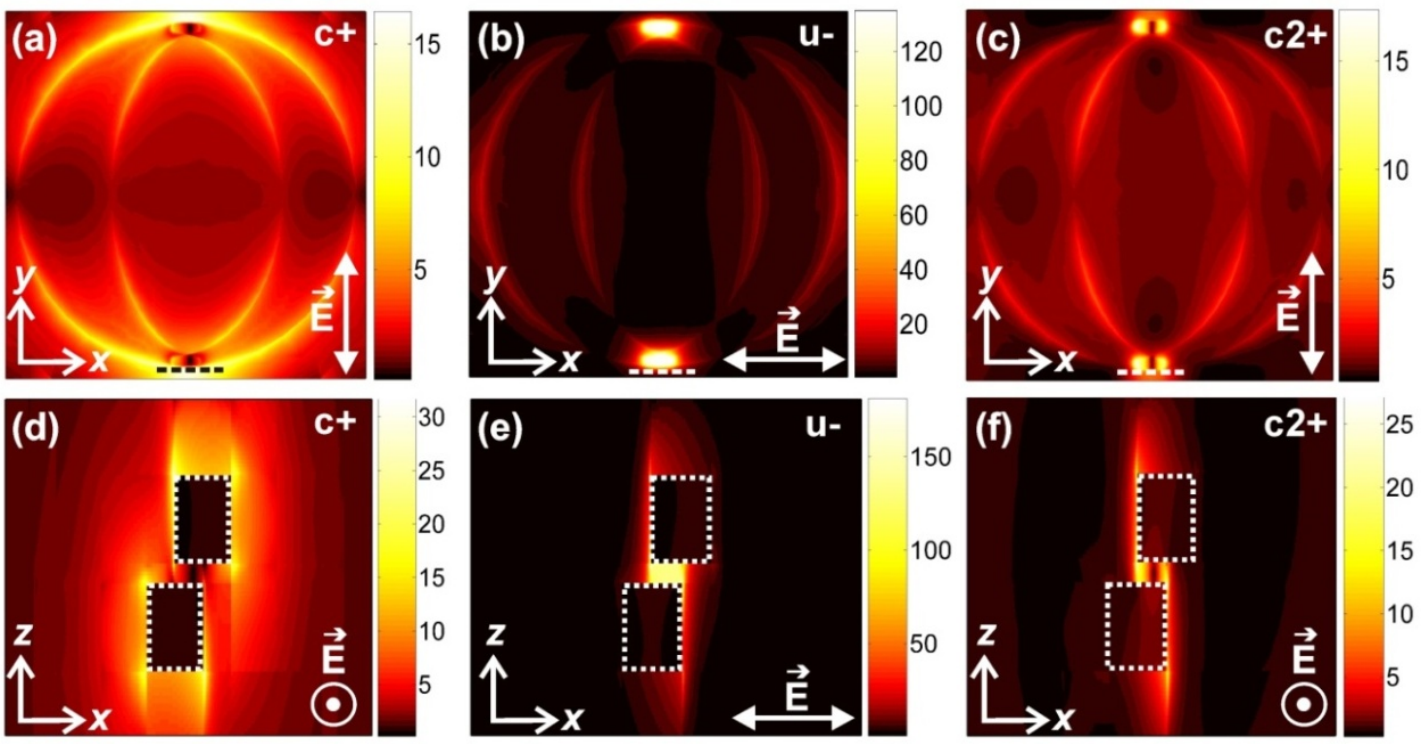

Figure 5.3.17. Near-field distributions of the stacked double crescents at resonance wavelengths. First row: near field in the xy-plane cutting half of the glass layer in between the crescents. Second row: near field in the xz-plane cutting the stacked structure along the tip region. The cut plane is indicated by dashed lines in the top row images. For better visibility, the crescents have been highlighted by a dotted frame. (a),(d) $c_{+}$resonance mode at a wavelength of $1690 \mathrm{~nm}$. The symmetric mode has similar charges at the tips of the crescents. Hence, no field enhancement in between the tips is visible. (b),(e) u. resonance mode at wavelength of $1750 \mathrm{~nm}$. The field enhancement in the overlap part is clearly resolved and shows that the tips are oppositely charged. (c),(f) $c_{2+}$ resonance mode at a wavelength of $910 \mathrm{~nm}$. The additional nodes of the mode are visible in the xy-plane. The tips have similar charges, leading to a depletion of the electric field in the middle of the gap between the crescents in the xz-plane. The enhancement visible at both sides of the gap is a result of the proximity of the tip charge and the opposite charges in the wings of the tips.

Figure 5.3.17a and $\mathrm{d}$ visualize the field distribution for the $\mathrm{c}_{+}$resonance at $1690 \mathrm{~nm}$. A strong field is present in the regions of the tips of the crescents, reflecting the areas with higher electron densities as predicted in the hybridization model. The node at the symmetry plane in the middle of the crescents is visible as well. In the gap between the tips of the crescents, no field enhancement is visible as the resonance is a symmetric mode and the tips have similar charges (see Figure 5.3.17d). The field distribution for the $u_{\text {. }}$ resonance at a wavelength of $1750 \mathrm{~nm}$ is presented in Figure 5.3.17b and e. The xy plane shows a strong field enhancement at the tips and a minor enhancement in the middle of the crescents. It agrees well with the charge distribution postulated by the hybridization model (Figure 5.3.14). Furthermore, the field is depleted at the wings of the crescents, thus imaging the two nodes of the wave function. As the resonance is antisymmetric, the tips facing each other bear opposite charges. Therefore, the electric field is strongly enhanced in the gap between the tips (Figure 5.3.17e). The hybrid $\mathrm{c}_{2+}$ mode at $910 \mathrm{~nm}$ is investigated in Figure 5.3.17c and f. Again, the field distribution agrees well with the theoretical model proposed. The cut in the xy plane shows a field distribution with three nodes resembling the sketch of the charge distribution given in Figure 5.3.16b. As the 
mode is symmetric and the tips have similar charges, no field enhancement should be visible in the gap between the tips. Figure 5.3.17f shows a depletion region in the middle of the gap while an enhancement is visible at the edges of the gap. The depletion visualizes the region where similar charges interact strongly. The field enhancement at both sides of the gap reflects the proximity of the tip charges to the opposite charges present at the wings of the crescents and supports the hybridization model for the $c_{2}$ resonance as established above.

\subsection{Conclusion stacked double crescents}

Concluding, a novel technique for the fabrication of large-area arrays of stacked double crescents by colloidal lithography is introduced. Sophisticated nanoscale objects can thus be aligned vertically with a precision of several nanometers, a feature that formerly required expensive, serial techniques as electron beam lithography or focused ion beam milling. The arrays feature a high degree of lateral symmetry that reflects the hexagonal order of the colloidal monolayer. The process is parallel, cheap and enables to structure large areas.

Ample degrees of freedom exist for the process that allow for precise adjustment of the nanocrescents' size, shape, overlap and mutual orientation. All of these characteristics have a strong effect on the optical properties of the objects and can be used to tailor plasmonic resonances according to the desired applications. Furthermore, the precise vertical alignment also gives access to observe fundamental aspects of the coupling process between nanostructures. Polarization dependent UV/Vis-NIR absorption spectroscopy showed that the close proximity in space led to drastic differences in the plasmon resonances compared to single crescents. In the presence of the second crescent, the fundamental plasmon mode blue-shifted while the energy of the first harmonic mode decreased. The effects were sufficiently strong that a cross-over between the two resonances took place, thus giving the impression that the first harmonic mode became energetically favored to the fundamental mode. A plasmon hybridization model was adapted to explain the resonance positions. Symmetry considerations showed that for each polarization, only one of the hybrid modes features a dipole and hence, is excited efficiently by the external electric field. As the symmetric situation for the fundamental and first harmonic mode is inverted, the shifts in energy for the stacked double structure are readily explained. A complete hybridization model for all polarization dependent resonances of nano-crescents dimers was elaborated, thus shedding light into the fundamental understanding of the behavior of complex structures in close proximity. Theoretical calculations reproduced all essential spectral features and thus, supported the proposed hybridization model. 


\subsubsection{Opposing double crescents with tunable plasmon resonances}

\subsection{Introduction}

Having successfully demonstrated plasmon hybridization in stacked crescent arrays, a modified construction procedure was elaborated in cooperation with Janina Fischer that led to two dimensional crescent dimers arranged in the substrate plane with tips facing each other.

The presented architecture combines several attractive features. First, it features a strong near field enhancement paired with a very low field volume. This is achieved by juxtaposing the sharp crescent tips in direct vicinity. Hence, the strong near field enhancement of the tips ${ }^{[259]}$ is effectively combined with a small gap that confines the electric near field to a very small volume. Additionally, the strong near field in the gap is addressable - and the controlled placement of functionalities in the hotspot feasible. This is seen as a significant development compared to the stacked crescent architecture introduced above that features a hotspot that is "hidden" in the matrix material separating the crescents.

The addressable hotspot makes the presented structure a promising candidate for novel surface enhanced Raman substrates and sensing devices. Fluorescence enhancement ${ }^{[260]}$ or plasmonmediated Förster resonance energy transfer ${ }^{[261]}$ could be investigated by addressing the gaps with fluorophores. Moreover, the presented double crescents possess dark modes as well, i.e. resonances without a net dipole that do not interact with the incident dipole ${ }^{[151-152]}$. Such modes are under intense investigation for the construction of metamaterials with unique optical features not occurring in nature. ${ }^{[242-243,249-250]}$

Finally, as the opposing double crescent structure is closely related to the stacked crescent architecture, it can also serve as a further verification of the plasmon hybridization model elaborated in the previous chapter.

The first part of this chapter introduces the modified fabrication process for crescent-shaped nanoparticles. This technique allows for precise shape-induced tuning of the plasmon frequency and leads to an extended understanding of the behavior of plasmon resonances in dependencies to variations of structural parameters. For a detailed elaboration of the hybridization behavior of the crescent dimers, the optical properties of single crescents produced by a similar process are investigated in the second part of the chapter. The process allows for precise shape-induced tuning of the plasmon frequency and leads to an extended understanding of the behavior of plasmon resonances in dependence to variations of structural parameters. In the third part, the method is extended to produce plasmonic dimer structures by the introduction of a second crescent in close proximity to the first one. Thus, tunable opposing double crescent structures 
with the tips facing each other are constructed and investigated with respect to the near-field enhancement in the gap between the tips that arises as a consequence of the strong coupling between the two crescents.

\subsection{Results and Discussion}

\section{Sample preparation}

The standard preparation method for gold nano crescent structures ${ }^{[129-130]}$ as presented in 5.3.2.1 uses an angular gold deposition followed by an reactive ion beam etching step normal to the surface. As a consequence, the gold reaches far underneath the areas shaded by the colloids crossing the center of the colloid. The construction of opposing double crescents by this method is cumbersome if not impossible as the wings of the two individual crescents overlap and form ring structures. In this way, the reference ring structures of Figure 5.3.13 of this chapter were formed.

To produce crescent structures that are in close proximity to each other but still separated, the existing preparation method was altered as schematically shown in Figure 5.3.18.

The gold evaporation step was performed normal to the surface (b) with a nominal thickness that corresponded to twice the height of the final crescents. A first reactive ion beam etching step was performed under an angle $\theta$ with respect to the surface normal (Figure 5.3.18c) to reduce the thickness of the gold film to half its initial size. The areas shaded by the colloids remained unaffected and now featured a crescent shape with a gold thickness of twice the height compared to the rest of the film. Subsequently, the sample was rotated $180^{\circ}$ and a second etching step was performed under an angle of $-\theta$ (d). Similar to the step before, the area shaded by the colloids remained unaffected while the continuous gold film was completely removed. As the area shaded by the colloids in the first step possessed a different height compared to the rest of the film, the two crescent structures appeared after the second etching step. Finally, the colloids were removed while the crescent dimer structures remained on the surface. As the two etching directions feature an angle of $180^{\circ}$ with respect to each other, the two crescents possess mirror symmetry (e). 


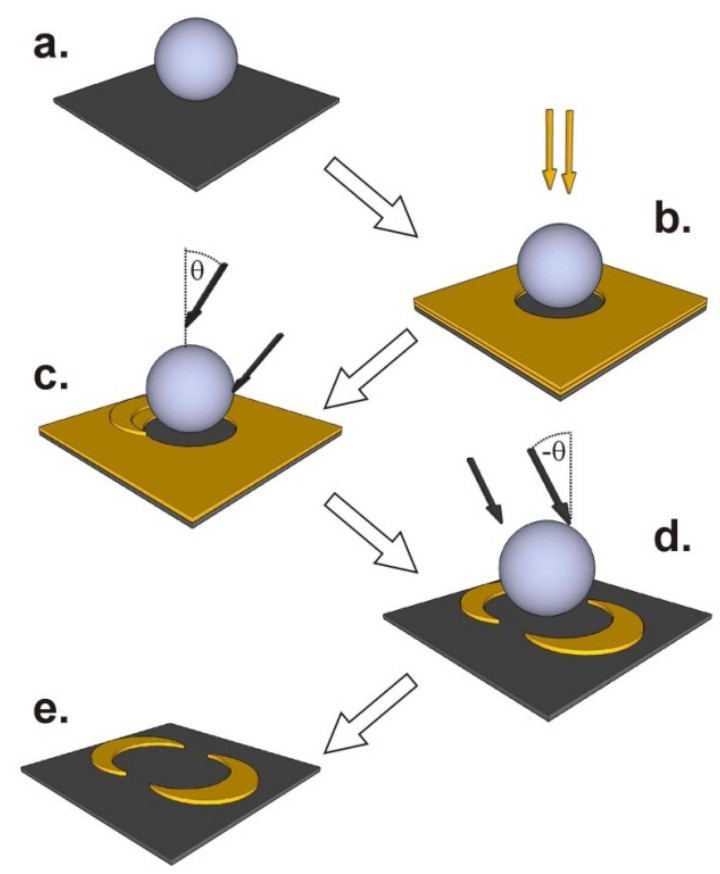

Figure 5.3.18. Fabrication scheme for opposing double crescents. A colloid serves as mask (a), gold is evaporated perpendicular to the surface (b), followed by two angular reactive ion beam etching steps (c and d) with an angle of $180^{\circ}$ with respect to each other. After removal of the colloid mask the double crescent structure remain on the surface (e).

Several parameters can be used to control the structures; most importantly the colloid size and the angle of the reactive ion beam etching steps. The latter introduces drastic changes in the crescents appearance as higher etching angle produces longer, more bulky crescents while small angles are used to produce structures that closely resemble the classical crescent shape, as e.g. shown in Figure 5.3.9. The process is limited to etching angles between approximately $40^{\circ}$ and $65^{\circ}$, as for smaller angles, the tips come into contact, and for very high angles, the crescents broaden and the structures' contours blur due to impreciseness of the colloids' shadows.

The structure of the individual crescents strongly affects their optical properties as the plasmon resonances are sensitive to minute changes of the geometry of the crescents. The dimer structures that can be created by the above-mentioned process feature tips in close proximity. Hence, strong coupling effects between the two crescents are expected. In order to examine the effects of the coupling, the optical properties and the influence of the geometry on the resonance position of the single crescent units have to be understood first.

For that, the process was adopted to produce single crescents by omitting the second reactive ion etching step. The optical properties of the single crescents prepared with different etching angles were investigated by UV/Vis spectroscopy and by further assessed computer simulations. A serious challenge for the interpretation of the resonance shift is the high sensitivity of the structures to minute changes in their shape that can occur due to imprecisions in the fabrication 
process. Hence, to avoid misinterpretations of the coupling process for the dimer structures, a model to correlate the geometrical parameters of the single crescents with their plasmon resonance frequencies has to be developed first. It is shown that each resonance correlates with a set of defined geometrical parameters. To understand the optical spectra of the double crescents, the electron microscopy images can be used to extract their exact geometrical parameters. These are subsequently used to calculate the resonance positions of a hypothetical, isolated single crescent with an identical shape using the model functions determined from the analysis of the single crescents. Finally, a comparison between the hypothetical, single crescent with the geometrically identical double crescent structure reveals the "real" shifts that are purely attributed to plasmon hybridization of the coupling process.

\section{Optical investigation of single crescents}

To gain fundamental understanding of the size- and shape dependent plasmonic properties, single crescents were produced in analogy to Figure 5.3.18 but without the second reactive ion etching step.

Figure 5.3.19a to e (upper parts) show the polarization-dependent extinction spectra of five different single crescent structures, prepared with different etching angles $\left(45^{\circ}\right.$ to $65^{\circ}$ tilting relative to the surface normal). The insets show the corresponding scanning electron microscope images. Computer simulations were performed by Reza Mohammadi in order to gain a better understanding of the plasmonic properties. Size and shape used for the calculations were adopted from the experimental structures. The simulated spectra are shown below the corresponding experimental spectra in Figure 5.3.19a to e. In all spectra, the black curves represent the c-polarized spectra, whereas the gray ones correspond to u-polarization. 
a.
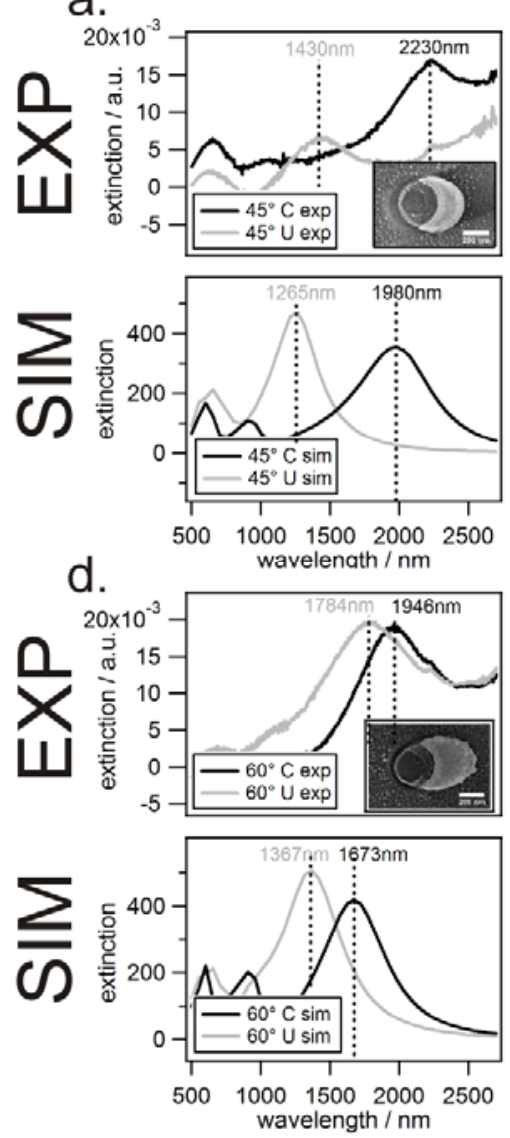

b.
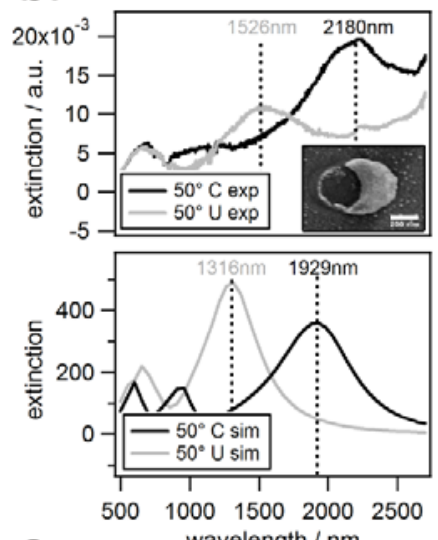

e.
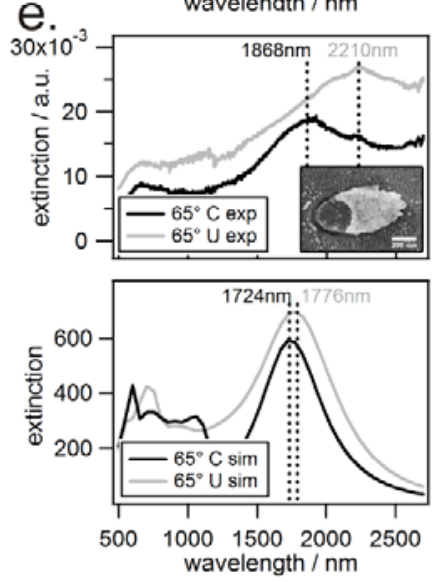

C.
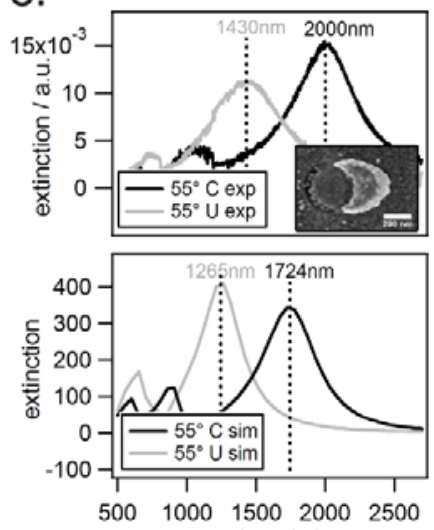

f. wavelength / $\mathrm{nm}$
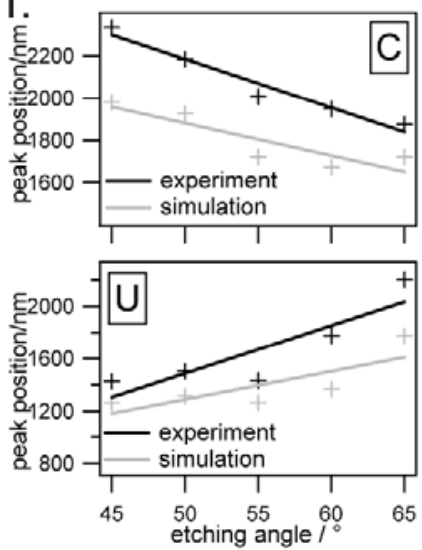

Figure 5.3.19. Extinction spectra of single crescents with different geometries (a. to e.). The upper graphs show the experimentally obtained data and the insets show the particular scanning electron micrographs. The lower graphs show the simulated spectra. f) Calculated and measured resonance peak positions for the applied etching angles for the $\mathrm{c}$ - and the u-resonance. The lines are guides to the eyes.

In Figure 5.3.19f the peak position dependence on the etching angle for both resonances of the simulated and the experimental curves are plotted. The qualitative features of the calculated shifts are in good agreement with the experimental data. The resonance wavelengths of the produced crescent structures strongly depend on the etching angle for both the $\mathrm{c}_{1}$ resonance (Figure 5.3.19k) and $\mathrm{u}_{1}$ resonance (Figure 5.3.191).

The experimental data summarized in Figure 5.3.19f reveals that the u-resonance undergoes a redshift of approximately $775 \mathrm{~nm}$ with increasing etching angle. In contrast, the c-resonance experiences a blueshift of almost $360 \mathrm{~nm}$. The change in resonances is caused by an increasing deviation from the typical "crescent moon" shape $\left(45^{\circ}\right.$ etching angle) towards a more distorted, bulky appearance ( $65^{\circ}$ etching angle) as is obvious from the SEM images presented in the insets of Figure 5.3.19. Most remarkably, the energetic positions of $\mathrm{c}$ and $\mathrm{u}$ resonance are inverted for the most distorted crescent structures (Figure 5.3.19e).

The simulated spectra qualitatively resemble the experimentally obtained data. However, all simulations yield smaller wavelengths than the experiment. This effect can be ascribed to the 
following differences between the experimental and the simulated spectra: First, roughness in the structures can cause significant shifts in the plasmon resonances. ${ }^{[238,258]}$ It has been shown that roughening of the structures induces a red-shift of the plasmon resonances, ${ }^{[238]}$ corroborating with the discrepancy between simulation and experiment as shown in Figure 5.3.19f.

Second, the determination of the exact geometry of the double crescent structure is difficult especially for those prepared with a very steep etching angle, as the structures get blurred due to beam inhomogeneities. This also holds for the thickness of the gold, which was assumed to exactly match the pre-set evaporation thickness. Lastly, no refinements of the simulation parameters, e.g. for the gold permittivity, were performed, as the simulations reflect all essential features of the experimental data. Adapting more precise models for the crescents, the quantitative agreement between simulation and experiment is expected to improve.

\section{Understanding the resonance positions}

To explain these striking shifts in the optical spectra, correlations between the peak positions and geometrical dimensions of the structures were analyzed in detail. First, the charge distribution at the resonance positions was imaged from the simulated data in order to ensure that the resonances in dependence on the polarizations (c- and $u$ polarization) are properly assigned and the model remains valid for all different stages of crescent distortion.

Figure 5.3.20 show charge distribution maps taken from the respective resonance positions of the simulated spectra for structures produced with etching angles of $45^{\circ}$ and $65^{\circ}$. The corresponding spectra and electron microscopy images are shown in Figure 5.3.19a and e. The two geometries were chosen as they represent the extreme cases of structures that can be generated by the process presented. While the $45^{\circ}$-structures show clear structural features of the typical nanostructure crescents, ${ }^{[129-130]}$ the $65^{\circ}$-objects possess a maximum degree of distortion. Figure 5.3.20a and $\mathrm{b}$ present the field distributions for the resonance detected in $\mathrm{c}-$ polarization. For both cases, the typical distribution of the fundamental plasmon mode is visible, featuring one node in the middle and high fields at the tips of the crescent, as indicated in the insets. The field distribution of the resonance appearing in u-polarization is shown in Figure 5.3.20c and d. Again, the typical distribution for the first harmonic is present for both cases. The mode features two nodes at the wings of the crescents and high fields at the middle and the wings of the structure and is schematically shown as insets. 

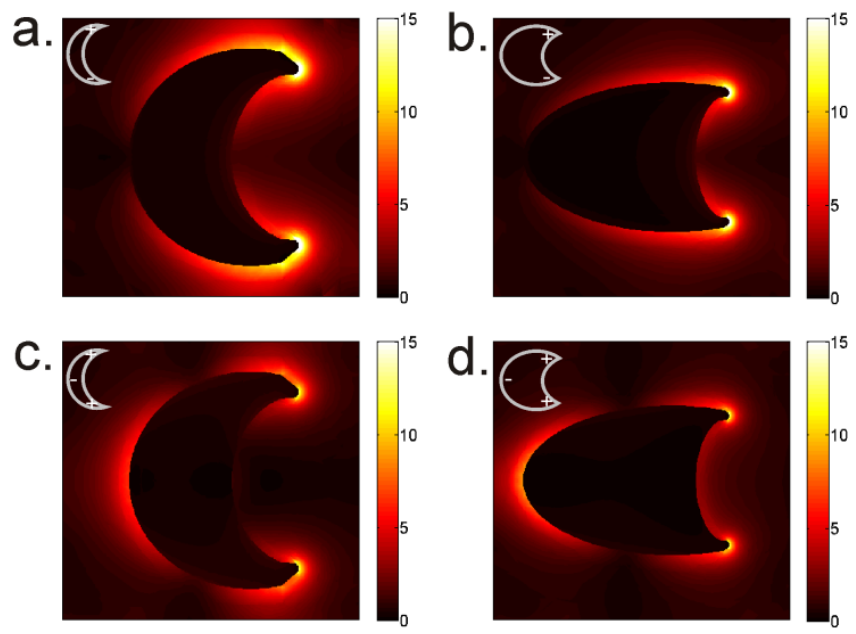

Figure 5.3.20. Charge distribution maps of the single crescent structures taken from the simulated spectra at the respective resonance positions. a,c) single crescent produced with a $45^{\circ}$ etching angle in c (a) and $u$ resonance (c). b,d) single crescent produced with an etching angle of $65^{\circ}$ in $\mathrm{c}(\mathrm{b})$ and u-polarization (d). The charge distribution as expected from the proposed model is as insets and is in good agreement with the field intensities shown in the simulations.

Concluding, it can be stated that the resonances of the crescents for all different etching angles can be accurately described by the established standard model. ${ }^{[129-130,148]}$ Apparently, the strong shifts in resonance positions - including the cross-over of resonances for the crescents produced with $65^{\circ}$ etching angle - have their origin in the special geometry of the crescents.

In order to predict the optical properties of the crescent structures, it is necessary to correlate the peak wavelength with geometrical parameters of the structure. Knowledge of this correlation is of crucial importance to tailor the resonance frequencies and to understand the coupling behavior of the dimer units, the characterization of which will be shown in the next chapter.

A model was elaborated to explain the shifts of the resonances based on geometrical factors influencing the respective resonances. Figure 5.3.21 illustrates the structural factors that are crucial for the shifts of the individual resonances as well as diagrams showing the good correlation between the simple geometrical features and the resonance positions. For the uresonance, a linear dependence of the resonance maximum on the crescent width is found (a). With increasing width, the peak shifts to longer wavelength. The behavior of the resonance is explained by a simple geometrical model based on the charge distribution at resonance conditions as displayed in Figure 5.3.21b. With increasing width of the crescent, the separation of the charges also increases, leading to a reduction of energy. The fit function for the dependency is given as inset in Figure 5.3.21a.

In contrast, the maximum of the c-resonance correlates with the contour length of the crescent. This parameter defines the distance between the two charges (Figure 5.3.21d). Upon increasing 
distance, the peak shifts to lower energies, as it defines the spacing between the charges. However, in the presented preparation process, a small contour length always corresponds to a big width of the crescent and vice versa. It has been shown in literature that upon increasing width of crescent-shaped nanoparticles, the c-resonance shifts to higher energies. ${ }^{[130,148]}$ Hence, both effects must be taken into account to obtain a reasonable correlation. Figure 5.3.21c shows the plot of the peak position of the $\mathrm{c}_{1}$-resonance versus the product of the contour length and the crescent width. A linear dependence is found, the fit function is given as inset.

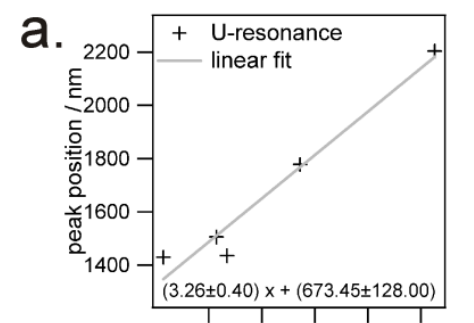

C.

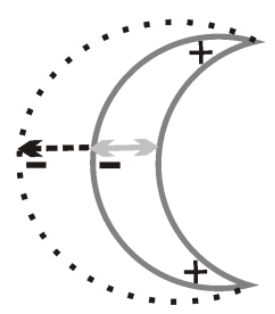
$250300 \quad 350$
crescent width 400

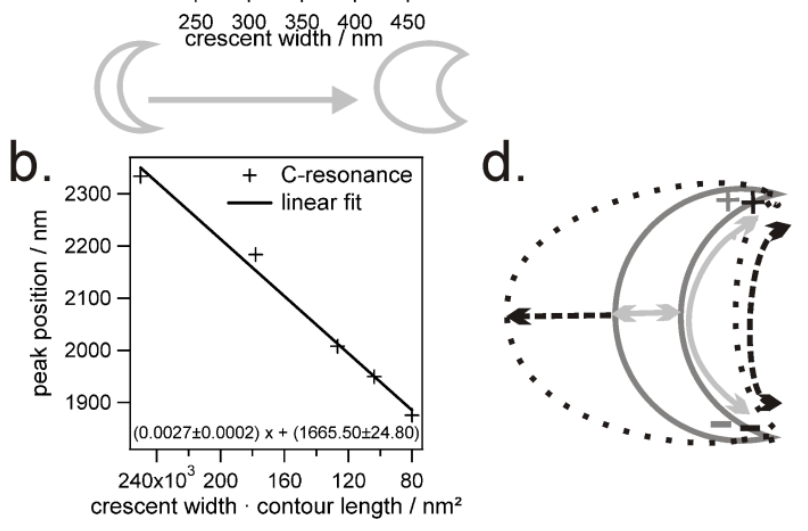

Figure 5.3.21. Geometrical factors that influence the resonance position . a) Dependence of the uresonance on the crescents' width. b) Dependence of the c-resonance on the product of the crescents' contour length and width. c) Effect of changes in the crescents' with on the charge distribution in the crescent at u-resonance. d) Charge distribution in the crescent at c-resonance upon variations in width and contour length.

These simple models for both resonances allow for explaining and predicting the shifts of the plasmon resonance upon minute changes in the shape of the nanostructure. The fit functions can be used to calculate the peak positions for both the $\mathrm{u}$ - and the c-resonance of any crescentshaped nanostructure with known shape by simply replacing the factor $x$ by the width or the product of the width and the contour length, respectively. The correlation between the simple geometrical factors and the plasmon resonance positions is surprisingly good and can thus be used to tailor resonances to desired wavelengths. 


\section{Plasmon hybridization in opposing double crescent structures.}

Having resolved the optical properties of the single crescents, the preparation step as shown in Figure 5.3.18 was completed to produce the opposing double crescent structures. As the tips are in close proximity, the electrical near-fields of the individual crescents are expected to overlap, leading to hybridization of the plasmon resonances.

Figure 5.3.22 shows the extinction spectra of the opposing double crescents as measured by polarization dependent UV/Vis-NIR spectroscopy. Representative electron micrographs of the opposing crescent dimer structures featuring the mirror symmetry of the structures are shown as insets.

Defined resonances are visible in both c- and u-polarization for all geometries of the dimer structures. The peak positions of the single and the double crescents' resonances with similar etching angles are plotted versus the etching angle in Figure 5.3.22f and g. Clear differences between single and double crescent structures are visible, thus indicating coupling processes between the individual crescents. A direct comparison between the resonances of single and double crescents reveals a blueshift in energy for the coupled resonances of the dimeric system for both c- and u-polarized light

a.

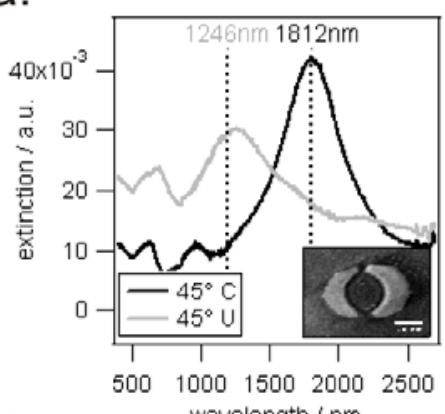

d.

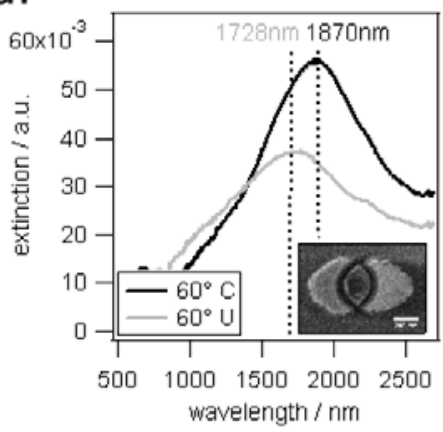

b.

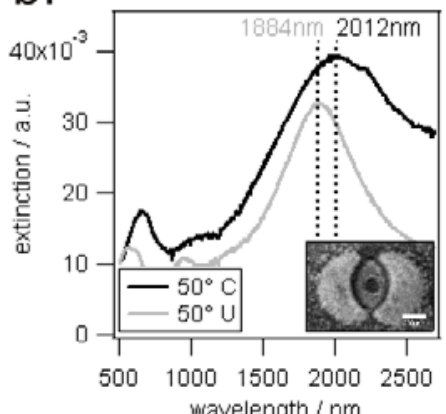

e.

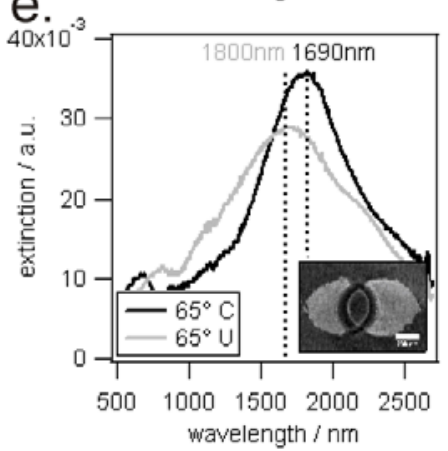

C.

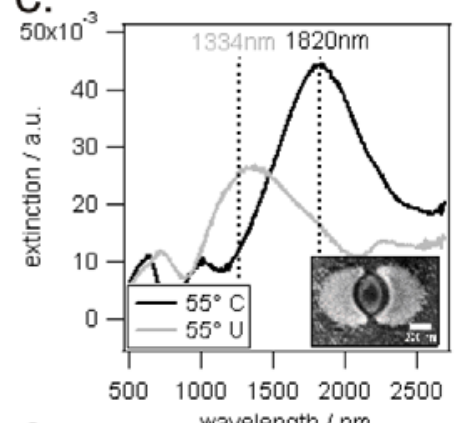

f.

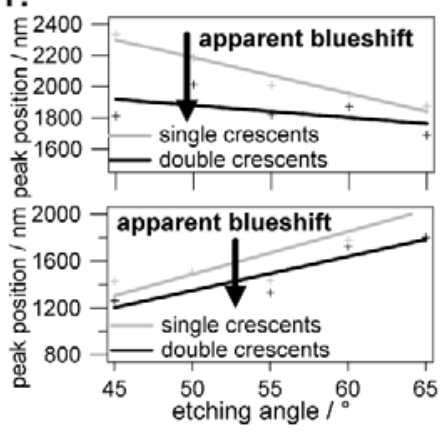

Figure 5.3.22. Polarization dependent UV/Vis-NIR investigation on opposing double crescent arrays produced with different etching angles. SEM images of the structures are shown as insets. a) $45^{\circ}$; b) $50^{\circ}$; c) $55^{\circ}$; d) $60^{\circ}$, e) $65^{\circ}$. Light polarization in c direction is shown as black lines, u-polarized light is shown in grey. f) Shows the shifts of the resonance wavelengths when directly compared to the measured spectra of single particles with the same etching angles. The lines are to guide the eyes. 
However, due to the fabrication process of the double crescents including the "blind" etching step for the first crescent shown in Figure 5.3.18, slight differences of the geometry compared to the single crescent structures may arise; especially the metal thickness and the exact shape of the crescents can vary between single- and double crescent structure for the different samples. The scanning electron microscope images reveal that the shapes of the double crescents indeed differ slightly from those of the single crescents (compare SEM insets in Figure 5.3.19 and Figure 5.3.22).

As established in the previous section, the resonances are very sensitive to minute changes of the crescents' shapes. Hence, the resonances of the double crescents cannot be directly compared to the single structures, but rather have to be analyzed in terms of their inherent geometric properties first.

By determining the exact geometry (i.e. width and product of width and contour length), it is possible to calculate the hypothetical resonance of a separated, single crescent with the geometry of the double crescents of the dimer unit. This is done by inserting the respective geometrical parameters of the crescent dimer structure into the fit functions elaborated for the single crescents (Figure 5.3.21). In detail, the mean crescent width of the double crescents in $\mathrm{nm}$ is inserted in the equation for the u-resonance (Figure 5.3.21a), namely $3.26 \mathrm{x}+673.45 \mathrm{~nm}$, where $\mathrm{x}$ represents the particular crescent width. To obtain the c-resonance, the product of the mean width and the contour length is inserted in the fit function of the singles crescents' cresonance, $0.0027 \mathrm{x} / \mathrm{nm}+1665.50 \mathrm{~nm}$ (Figure 5.3.21c), with $\mathrm{x}$ being the particular product of the width and the contour length. This yields the theoretical resonances for single crescents with exactly the geometries of the fabricated double crescents. It also corresponds to the resonance of the double crescents if no coupling took place. Therefore, in the following, it is termed uncoupled resonance. This procedure eliminates misinterpretations of the coupling process caused by direct comparison of single and double crescent samples produced with the same etching angle. The different samples are not necessarily identical due to imperfections in the production process as their geometrical parameters are subject to slight sample-to-sample variations.

To obtain the coupling-induced shifts of the double crescents, the experimentally determined resonance positions of the double crescent dimers are compared to the calculated uncoupled values. The procedure reveals the "real" shifts which arise due to plasmon coupling and are presented in Figure 5.3.23. The experimental values of the c-resonance of the double crescents are blueshifted when a second crescent is introduced (a). In contrast, the u-resonance experiences a redshift for all double crescent samples produced (b). 

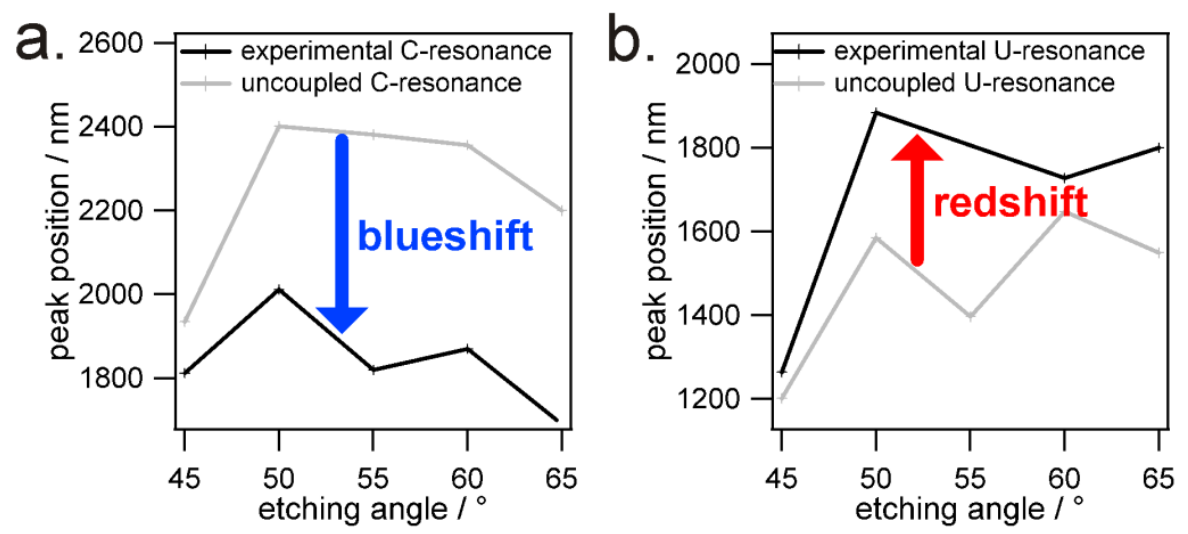

Figure 5.3.23. Coupling-induced shifts of the c- (left side) and the u-resonance (right side) of the opposing double crescents. The grey curves represent the shape-corrected data as calculated from the geometric data and the fit functions determined for the single crescents, the black curves are assigned to the experimental data of the coupled double crescents.

The shifts of the resonances as purely arising from the coupling behavior support by the plasmon hybridization model established for the stacked double crescent structures. In analogy to the stacked crescents situation, a hybridization model is straightforwardly obtained by taking linear combinations of the individual crescents' resonances. Figure 5.3.24 shows simplified sketches of the hybridization behavior.

a)
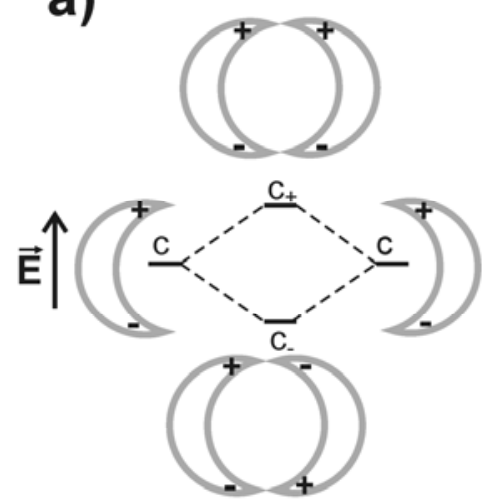

b)

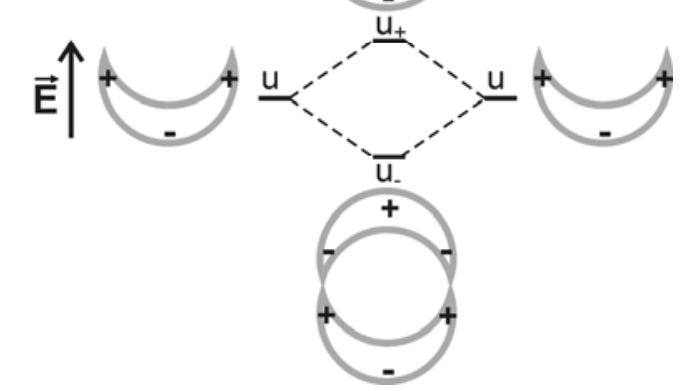

Figure 5.3.24. Plasmon hybridization model used to explain the coupling behavior of the crescent resonances. Upon close proximity, hybrid resonances appear as linear combinations of the parental crescent resonances. a) Geometrical situation in c resonance. b) Geometrical situation for the u resonance.

In c-polarization, only the symmetric hybrid mode $\mathrm{c}_{+}$shows a net dipole and can couple to external electric fields. Hence, only this mode is visible in optical spectra. Due to the higher energy, the resulting resonance appears blueshifted. The antisymmetric mode $\mathrm{c}$. does not feature a net dipole and, thus, represents a dark mode. ${ }^{[151-152]}$ In u-polarization, the situation is inverted due to the different charge distribution geometry between the two resonances. Here, only the 
antisymmetric mode $\mathrm{u}$ - exhibits a net dipole and can thus be excited by linear polarized light. The symmetric hybrid $\mathrm{u}^{+}$, however, is a dark mode and does not appear in the spectra. As the energy of the antisymmetric u- hybrid is lower than the energy of the single structure, the resonance appears redshifted compared to the parent resonance of the individual crescents.

As presented in Figure 5.3.23, both c- and u-resonances of the double crescent architectures are shifted according to the hybridization model for all samples produced. This indicates that a strong coupling of the individual resonances takes place in the dimer units. Quantitatively, the coupling efficiency varies between the different samples (compare Figure 5.3.23). We attribute this to differences in the separation of the tips of the two crescents. As apparent in the SEM images in Figure 5.3.22, the tip separations vary. If the tips are further apart from each other, the overlap of the electrical fields is reduced, thus, the coupling efficiency is decreased.

\section{Computer simulations to investigate the near-field enhancement}

Computer simulations have been performed for the double crescent structures with the aim to support the hybridization model by imaging the charge distributions at the hybrid resonance positions and to quantify the near-field enhancement in the gap between the opposing crescent tips. The crescent structures with $45^{\circ}$ and $65^{\circ}$ etching angle were exemplarily chosen as these represent the two extreme cases of distortion.

Figure 5.3.25 shows the electrical near-fields of the c- and the u-resonances of the single crescents and the corresponding double crescent structures for different tip-to-tip gap distances. The c-resonances of both the $45^{\circ}$ and the $65^{\circ}$ double crescents show a depletion of the electrical near-field in the tip region, indicating the presence of two equal charges which reject each other. In contrast, the u-resonances show an enhanced electrical near-field in the gap between the tips, pointing towards the fact that the tips exhibit different charges. The charge distributions closely resemble the simplified sketches of the proposed model (Figure 5.3.24) and thus strongly support the established hybridization theory.

Finally, the near-field enhancement of the dimer architectures at the resonance wavelengths was assessed by computer simulations and compared to the enhancement of single crescents. The geometry, featuring two sharp tips separated by a small gap promises to show a strong enhancement of the near-field that is confined to the small volume of the gap. 

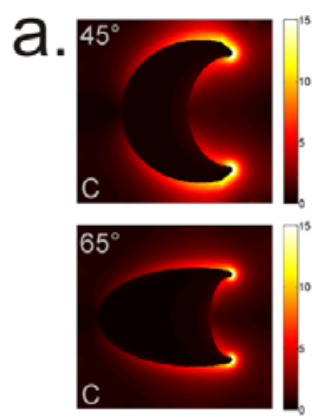

C.
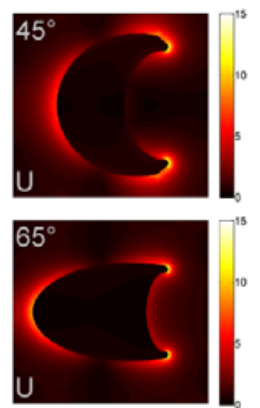
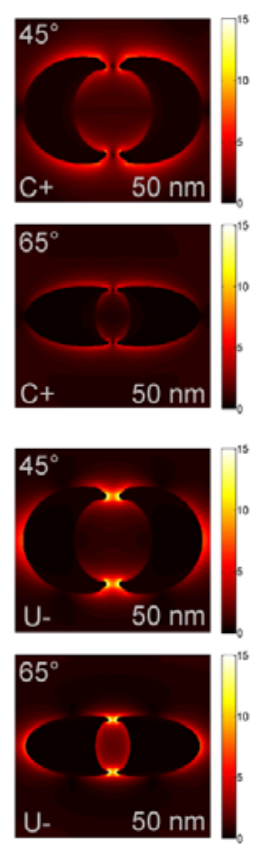
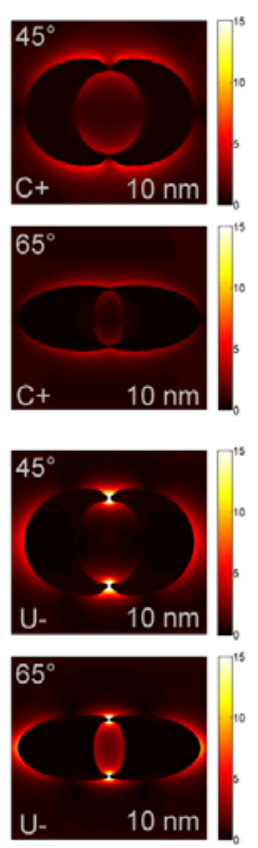
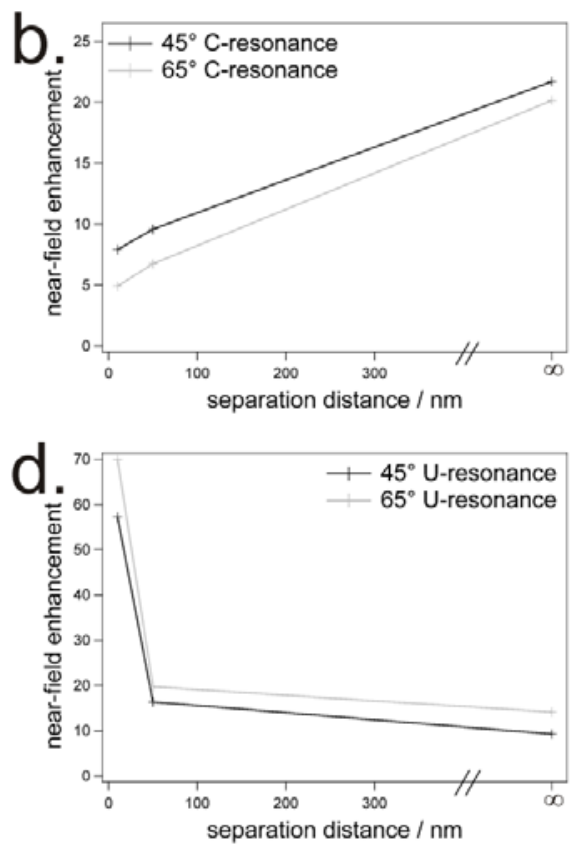

Figure 5.3.25. a) Simulations of the electrical near-fields in c-polarization of single crescents and opposing double crescents for $50 \mathrm{~nm}$ and $10 \mathrm{~nm}$ tip separation distance (upper row: $45^{\circ}$ etching angle, lower row: $65^{\circ}$ etching angle). b) Corresponding maximum near-field enhancements in c-polarization of

double crescents versus the separation distance. c) Simulations of the electrical near-fields in upolarization of single crescents and opposing double crescents $50 \mathrm{~nm}$ and $10 \mathrm{~nm}$ tip separation distance (upper row: $45^{\circ}$ etching angle, lower row: $65^{\circ}$ etching angle). d) Corresponding maximum near-field enhancements in u-polarization of double crescents versus the separation distance.

Figure 5.3.25b and d show the near-field enhancements for c- and u-resonances plotted versus the separation distance of the crescents. The value of the near-field of the single crescent reference is inserted in the plot as infinite distance for comparison.

As expected, the near-field is depleted for the double crescent architectures in c-resonance (b) and the enhancement is lowered from a value of approximately 20 for the single crescents to less than 5 for double crescents with a tip separation of $10 \mathrm{~nm}$. In contrast, a strong near-field enhancement is revealed in u-resonance. For single crescents at u-resonance, the enhancement factors are 15 ( $65^{\circ}$ etching angle) and 10 ( $45^{\circ}$ etching angle). The difference is readily explained by the higher tip curvature of the $65^{\circ}$ crescent. By introducing a second crescent in close proximity, this near-field enhancement can be increased up to a factor of almost 60 for the $45^{\circ}$ sample. For the $65^{\circ}$ structure, the near-field enhancement can even be increased up to a factor of 70. Hence, the electrical near-field of the dimer structures is five times stronger than the near-field of the individual crescents. This offers two precisely localized, connected hotspots that can be accessed by functional molecules due to the planar arrangement of the two crescents. Various applications such as plasmon-mediated Förster transfer, surface-enhanced Raman spectroscopy, or fluorescence enhancements are thus feasible. The structures also hold great promise to be used as novel sensing devices or metamaterials. 


\subsection{Conclusion}

This chapter describes a modified fabrication process for crescent shaped nanoparticles. By inversion of the angles of gold evaporation and reactive ion beam etching compared to the standard process (Figure 5.3.8), this method is suited for the construction of dimeric units with their tips facing each other.

The optical properties of single crescents prepared with different etching angles - and thus geometries - were investigated and supported by computer simulations. Correlations between geometric parameters and the plasmon resonance position were elaborated for both polarizationdependent resonances, revealing a strong influence of the crescent width on the u-resonance, and of the product of width and inner contour length on the c-resonance, respectively. This correlation can be used to precisely predict the resonance positions for a given geometry and provide a valuable tool for understanding and tailoring the individual resonances.

Bringing two individual crescents in close proximity, their electrical near-fields overlap and lead to a hybridization of the individual plasmon resonances. The resonances of opposing double crescents experience strong shifts as a result of two distinct reasons. First, the individual shape of the structures causes a specific shift as the resonance is very sensitive to minute changes in the crescents' geometry. These shape-induced shifts cloak the effects caused by the coupling of the plasmon resonances. The shape-induced shift was corrected by calculating the resonance of a theoretical, uncoupled crescent using the correlations between geometry and position elaborated for the single crescents. Thus, the plasmon shifts evoked solely by the coupling process were revealed. A plasmon hybridization model was applied to explain the shifts of the resonances. Theoretical investigations on the near-field distribution further corroborated with the model proposed.

The double crescent architecture combines sharp tips with a small separation gap. These geometrical features provide an ideal architecture for strong near-field enhancements in the hotspot between the crescent tips. The planar architecture provides the opportunity to access this hotspot. This opens the possibility for the utilization of the double crescents in surface enhanced Raman scattering, fluorescence enhancement or plasmon mediated Förster resonance energy transfer along the crescents' contours. Using computer simulations, the maximum additional enhancement of the near-field was determined to be five times as high as the enhancement for single crescent structures. 


\subsubsection{Laterally patterned, ultraflat substrates by colloidal lithography and their applications}

This chapter describes applications of ultraflat substrates consisting of gold nanostructures created by colloidal lithography embedded in a silicon dioxide matrix. The process takes advantage of the natural flatness of a silicon wafer that acts as a template for the structure deposition. In a procedure known as template-stripping, the structures created are cleaved from the template. Thus, the flatness of the template substrate is transferred to the newly created surface. The process itself was established during my diploma thesis. ${ }^{[262]}$

In continuation of this work, two applications of such patterned, ultraflat substrates were investigated. First, the localized surface plasmon resonance of the gold nanostructures was used to create extremely stable sensor architectures that could be re-used by a simple mechanical cleaning procedure. Second, thiole terminated polymerizable lipids, synthesized by Dr. Mathieu Jung, ${ }^{[263]}$ were assembled on the gold nanostructures and polymerized to form impenetrable diffusion barriers. A lipid bilayer membrane was subsequently assembled and thus featured nanoscale patterns embedded in the proximal leaflet of the membrane. Fluorescence recovery after photobleaching (FRAP) experiments were performed to investigate the effect of the nanoscale barriers on the membrane dynamics. Finally, isolated silicon dioxide patches corraled by a continuous gold film were produced in order to immobilize complete membrane fractions onto a designed spot. The two applications of laterally patterned, ultrafast substrates are published as "Reusable Localized Surface Plasmon Resonance Sensors Based on Ultrastable Nanostructures" ${ }^{[264]}$ and "Nanoscale Patterning of Solid Supported Lipid Bilayer Membranes by Integrated Diffusion Barriers". [265] 


\subsubsection{Introduction: laterally patterned, ultraflat substrates}

Laterally patterned, ultraflat substrates are surfaces that are chemically structured (i.e. consisting of two different materials) but do not feature any surface topography or defects in the transition between the different surface materials. The construction of such architectures was established during my diploma thesis ${ }^{[262]}$ and published ${ }^{[188]}$ and shall be outlined only briefly for reasons of clarity.

The process is based on a modified template-stripping procedure that was introduced by Butt ${ }^{[266-}$ ${ }^{267]}$ and Hegner ${ }^{[268-269]}$ independently. A naturally flat surface such as Mica or a polished silicon wafer is used as a template onto which gold is evaporated. After gluing a solid support (e.g. a microscope slide) onto the gold surface, the low adhesion between gold and silicon is used to cleave the interface. Thus, a new, "template-stripped" surface is exposed and the low roughness of the template is transferred to the gold surface.

Template-stripped gold substrates find various applications where conducting surfaces with a surface roughness below the dimensions of the studied objects are needed. Originally developed for scanning tunneling microscopy imaging, ${ }^{[266-269]}$ these substrates have been extensively used as electrodes for the electrochemical characterization of self-assembled tethered lipid bilayer membranes ${ }^{[270-272]}$ Recently, similar surfaces have been employed for the construction of high quality plasmonic materials ${ }^{[273-274]}$ and as substrates for the patterning of graphene. ${ }^{[275]}$

Using two different surface materials in a similar approach adds to the versatility of the produced substrates as different properties of the patterning materials can be exploited individually. Choosing gold and silicon dioxide as surface materials provides a simple means to create highly functional surfaces as gold- and thiole chemistry can be employed simultaneously to incorporate functions at defined places of the substrate. ${ }^{[188]}$ Furthermore, using colloidal monolayers as masks, a simple method for a nanoscale patterning is at hand and the plasmonic properties of the resulting gold nanotriangles can be exploited in sensing applications. ${ }^{[264]}$

The construction of such heterogeneously patterned substrates using a colloidal monolayer as mask is briefly outlined in Figure 5.3.26. A hexamethyldisilazane-modified silicon wafer was used as a template. A colloidal monolayer was assembled on the template (a) and used as mask for the deposition of layers of gold $(50 \mathrm{~nm})$ and chromium $(<2 \mathrm{~nm})$ to produce typical nanostructure arrays (b). The colloidal mask was then removed (c), and silicon dioxide $(100 \mathrm{~nm})$ was evaporated over the surface and completely covered the patterns (d). Subsequently, a glass slide was glued onto the silica, and a rupture between the passivated template (silicon wafer) and the evaporated structures was mechanically induced (e). A pristine, 
flat surface was obtained with gold nanostructure arrays with square-centimeter dimensions embedded in a silicon dioxide matrix. A detailed characterization of such surfaces showed extremely low roughness in the order of the silicon wafer template, smooth transitions between gold and silicon dioxide and the possibility of independent surface functionalizations. ${ }^{[188]}$

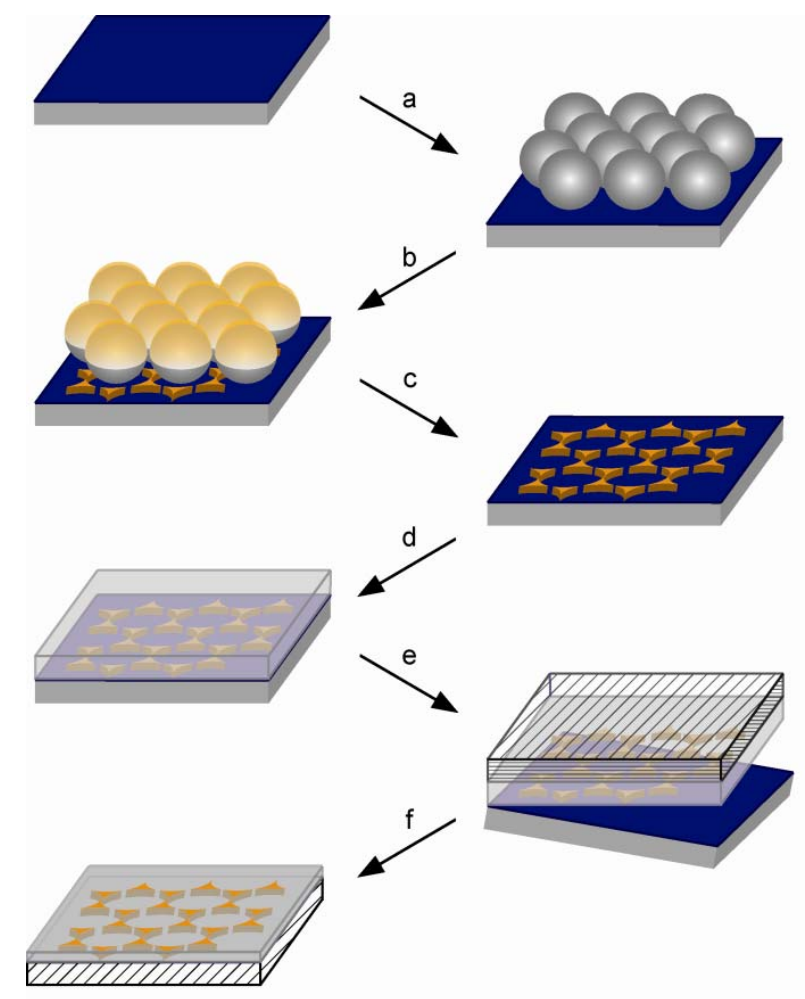

Figure 5.3.26 Flow process for the construction of embedded nanoparticles arrays: A hexamethyldisilazane-modified wafer is used as template material. a) Colloidal monolayer assembly. b) Thermal evaporation of gold $(50 \mathrm{~nm})$ and chromium $(<2 \mathrm{~nm})$. c) Removal of the colloidal monolayer. d)

Thermal evaporation of silicon dioxide $(100 \mathrm{~nm})$. e) Gluing of the interface to a glass slide and mechanical separation. e) Template-stripped surface with gold nanoparticles embedded in a silicon dioxide matrix.

\subsubsection{Re-usable sensing architectures}

\subsection{Introduction}

In the context of this thesis, a major focus is laid on surface bound nanoparticles arrays as created by nanosphere lithography ${ }^{[5,276]}$ that employs colloidal monolayers as patterning masks. 
A tremendous knowledge has been accumulated on their structural design, optical properties, and sensing capabilities. ${ }^{[123-124]}$

As serious drawback for the commercial use of such nanoparticles arrays is their insufficient stability in aqueous environments. Especially in buffer solutions, commonly used in bio-assays, metal nanoparticles tend to easily get washed off the surface. ${ }^{[277-278]}$ Only recently, this problem has started to attract attention and first solutions have been proposed. ${ }^{[45,279]}$ Nanoparticles have been anchored to small holes in the substrates and the decrease in signal intensity over one hour improved from $50 \%$ loss in untreated samples to $13 \%$ loss for surface anchored particles. ${ }^{[279]}$

Next to stability, the reusability of the architecture is important for possible applications, however rarely discussed in literature. Few approaches for regenerating surfaces based on reversible binding of molecules have been reported, ${ }^{[278,280]}$ nevertheless, no general cleaning methodology has been described.

In this chapter, the stability of surface embedded nanoparticles produced according to Figure 5.3.26 is investigated. It is found that they exhibit a superior stability in aqueous environments and can be mechanically cleaned and re-used in simple sensing experiments.

\subsection{Results and discussion}

\section{Stability in aqueous solutions}

In order to evaluate the stability of the embedded particle arrays, the substrates were exposed to a PBS buffer solution under a constant flow rate of $1.5 \mathrm{ml} / \mathrm{min}$. The integrity of the samples was followed in time by UV/Vis-NIR transmission spectroscopy (Figure 5.3.27). The obtained extinction data was normalized to the relative peak height $\left(\mathrm{I}_{0, \mathrm{LSPR}}\right)$ before exposure to the aqueous environment (Figure 5.3.27b). For comparison, samples of conventionally produced, non-embedded nanoparticles on a glass substrate with and without a $2 \mathrm{~nm}$ chromium adhesive layer between the glass surface and the gold particles were investigated (Figure 5.3.27b). Nonembedded, conventional nanoparticles arrays showed a strong decay in signal intensity. After $20 \mathrm{~h}$ of immersion, the extinction decreased to $7.5 \%$ of its original value. The sample with a chromium interlayer only showed a slight loss of signal intensity. After several hours, the signal dropped below $90 \%$ of the original value. The embedded particles, however, did not show any loss in signal intensity even after 5 days of incubation. Exposure to ultra-pure water instead of buffer solution revealed the same stability for the embedded particle arrays (not shown). 
To further investigate the stability of the different particle architectures, the chromiumcontaining samples were cleaned mechanically by wiping the surface with a tissue soaked in ethanol and water. This drastic procedure revealed a superior robustness of the embedded particles as no change in signal intensity was detected (Figure 5.3.27c) after the cleaning. The conventional particle array, however, was entirely removed and no plasmonic resonances were detected after the cleaning procedure.
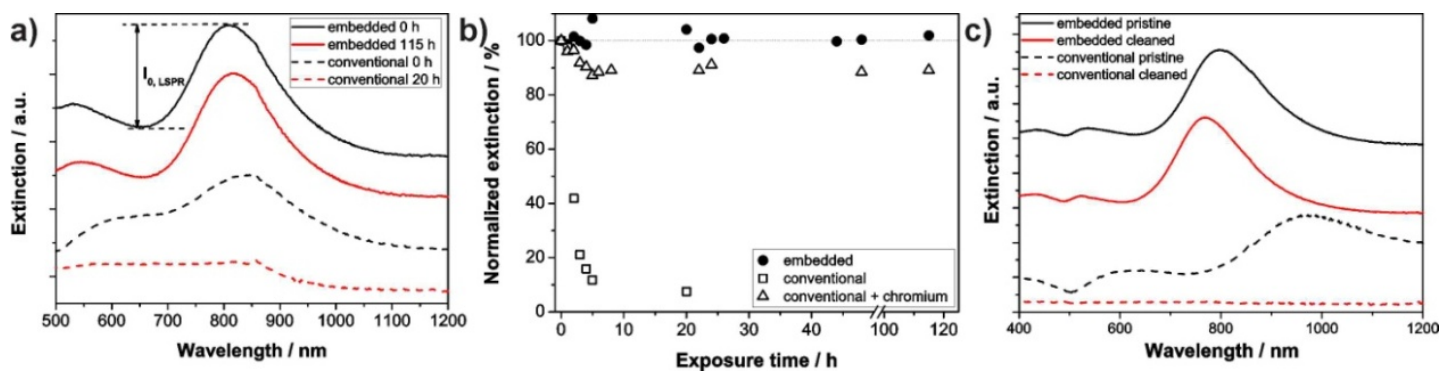

Figure 5.3.27. Stability of nanoparticles arrays investigated by UV/Vis-NIR spectroscopy. a) Plasmonic resonances of conventionally produced and surface embedded nanoparticles arrays upon exposure to PBS buffer solution. b) Stability of nanoparticles arrays during exposure to PBS buffer solution normalized to the resonance intensity before exposure. c) Plasmonic resonances of surface embedded gold particles (50 nm thickness) and conventionally produced gold nanoparticles ( $30 \mathrm{~nm}$ thickness) with a $2 \mathrm{~nm} \mathrm{Cr}$ adhesion layer between glass and gold before (black lines) and after mechanical cleaning (red lines).

\section{Sensing of thin polymeric layers}

In order to probe the response of the embedded particles to changes in their local environment and their potential use for sensing applications, thin polyelectrolyte films were deposited using a layer-by-layer (lbl) deposition method. ${ }^{[281-282]}$ A particle array was produced by using a monolayer of $550 \mathrm{~nm}$ diameter polystyrene colloids as mask. Samples were functionalized by immersion in an aqueous cysteamine hydrochloride solution for $90 \mathrm{~min}$. Afterwards, layers of negatively- (poly(styrene-sulfonate), PSS) and positively charged (poly(allylamine hydrochloride), $P A H$ ) polyelectrolytes were deposited, and UV/Vis-NIR spectra were recorded in air. Typical plasmonic resonances were observed (Figure 5.3.28a) and a red shift of the extinction maximum was induced upon increasing thickness of the deposited polyelectrolyte layers.

The red shift upon increase in polymer layer thickness can be quantified by extraction of the peak extinction wavelength (Figure 5.3.28b). Apart from the shift, no major changes of the plasmon resonance spectra were visible, indicating stability of the particle arrays towards the experimental conditions. The complete LSPR shift response to material deposition showed a 
non-linear behavior as reported for conventionally produced particles. ${ }^{[46]}$ The initial linear shift decreased after addition of 5-6 bilayers until saturation was reached and no additional shifts of the resonance spectra were detected upon deposition of further layers.

The response of the nanoparticles to the addition of polyelectrolyte layers was estimated from the peak displacement in the initial linear regime. The maximum extinction wavelength shifted on an average by $5.2 \mathrm{~nm}$ per deposited layer, for the first six bilayers (or 12 alternating single layers). The apparent saturation regime after six bilayers correlated to a total polyelectrolyte thickness of approximately $50 \mathrm{~nm}$ as estimated from surface plasmon resonance spectroscopy measurements on equally treated gold films. ${ }^{[148]}$ The maximum peak displacement between the untreated sample and the one modified with six (or more) polyelectrolyte bilayers was about $65 \mathrm{~nm}$, which is in good agreement with results reported for conventionally produced particles. ${ }^{[46]}$
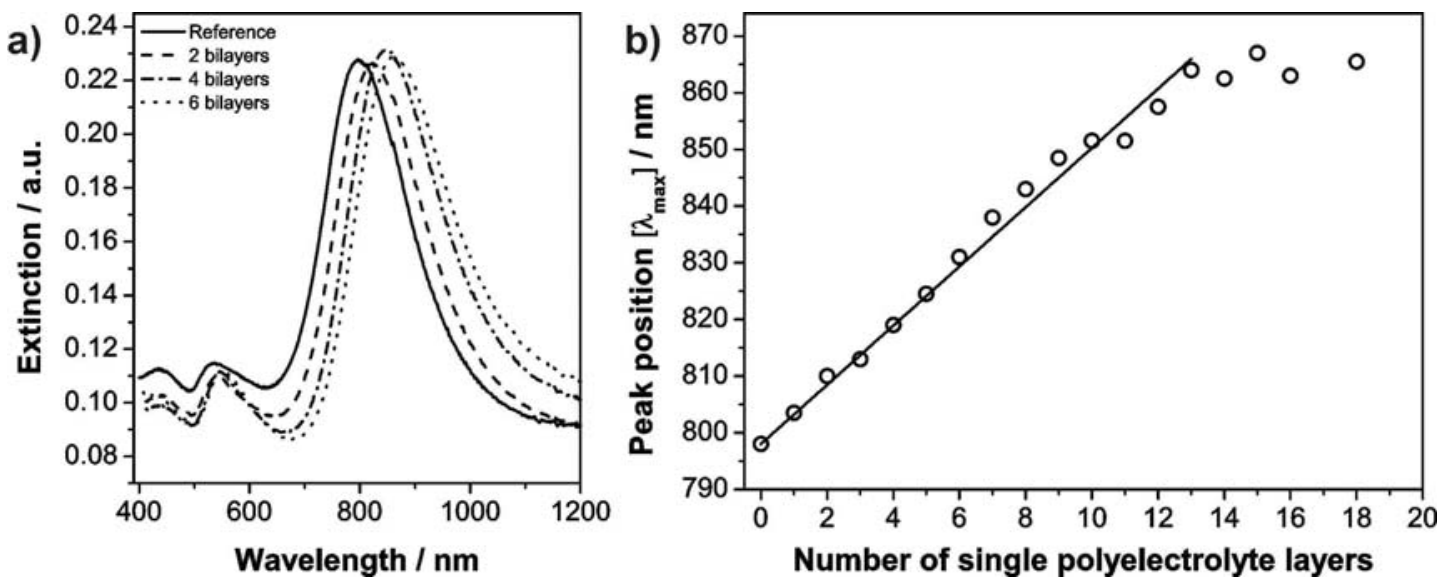

Figure 5.3.28. a) Plasmon resonance spectra of the particle array during polyelectrolyte deposition. b) Resonance position as a function of the number of polyelectrolyte layers. The shift is linear for the first 6 deposited layers before an apparent saturation is reached.

\section{Reusability}

The deposited polyelectrolyte layers were successfully removed by repeated, gentle wiping the substrate with a tissue soaked in ethanol and water. AFM images were recorded in order to monitor the surface during the cleaning process (Figure 5.3.29). After cleaning the sample, the original nanostructured surface was completely recovered without any traces of the polyelectrolyte layers remaining on the substrate (Figure 5.3.29d). A slight increase in height of the nanostructures can be attributed to the deposited cysteamine layer that is not - or not completely - removed by the mechanical cleaning. However, no damage or loss of structures was induced even by the rather harsh cleaning procedure, showing the stability of the substrates. 
a)
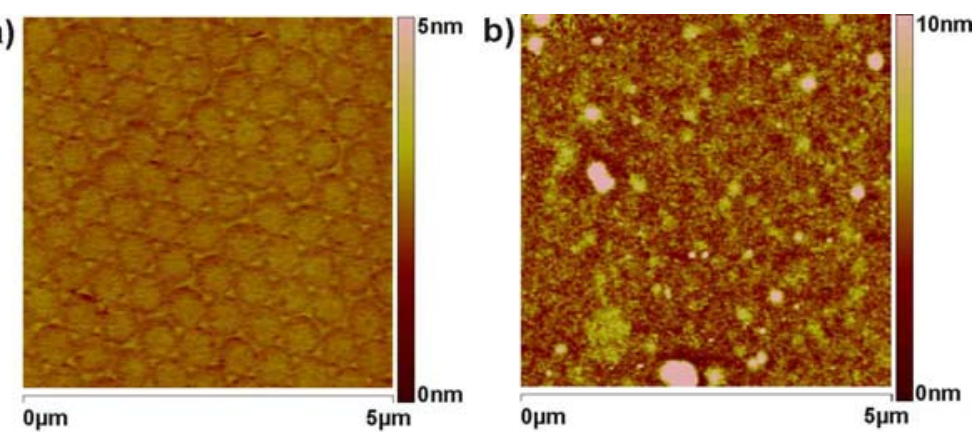

c)
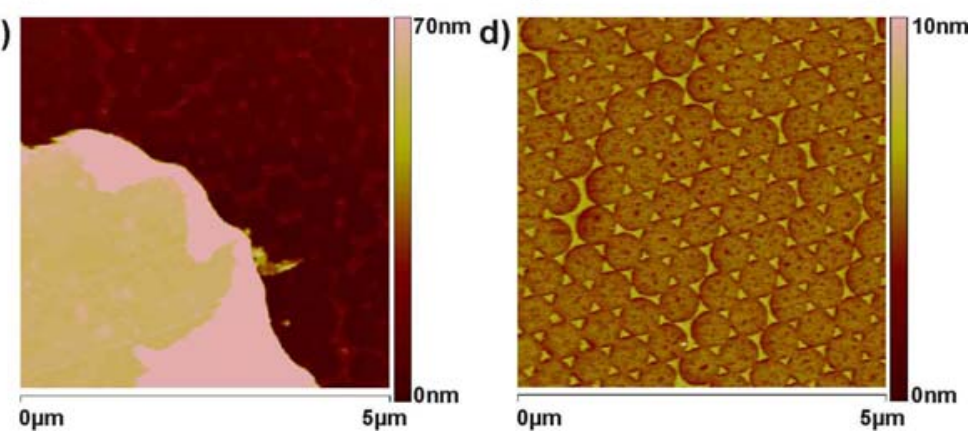

Figure 5.3.29. AFM-height images of nanoparticle arrays. a) Freshly cleaved substrate. The surface hardly shows any topography. b) After deposition of eight polyelectrolyte bilayers, the complete substrate is covered with the polymer film. c) The polyelectrolyte film can be mechanically removed. In the lower left corner, a still intact film is visible, while in the top right corner, the embedded nanostructures become visible. d) On a completely cleaned substrate, the embedded nanoparticles are again visible.

The cleaned sample was used for further consecutive polyelectrolyte deposition-cleaning cycles to explore the re-usability of this kind of sensing platforms. UV/Vis-NIR spectroscopy was used to monitor the effects of the deposition-cleaning cycles on the plasmonic resonances of the embedded nanoparticles. The peak displacements upon polymer addition evolved similarly to the original sample. The shift of the maximum extinction wavelength was extracted after each deposited layer for a substrate subjected to consecutive layer by layer-deposition-cleaning cycles (Figure 5.3.30). For each cycle, at least five polyelectrolyte bilayers were deposited on the surface before the sample was cleaned mechanically. Deposition cycles four and five were done after storing the substrate for 4 months at lab conditions. For each cycle, the substrate was incubated in a cysteamine solution to guarantee that initial charges are present on the surface. While a red shift of five nanometers was visible after the assembly of the cysteamine monolayer on the pristine gold surface, almost no shift was detected for all measurements on cleaned substrates, indicating that the cysteamine layer was not- or not completely removed by the cleaning process as also shown by AFM investigation (Figure 5.3.29). The maxima of the plasmon resonances red shifted as a function of deposited layers for all five deposition-cleaning cycles. The slope of the shift curves remained approximately constant at a value of $10-13 \mathrm{~nm}$ per bilayer during all cycles, indicating the re-usable sensing abilities. 


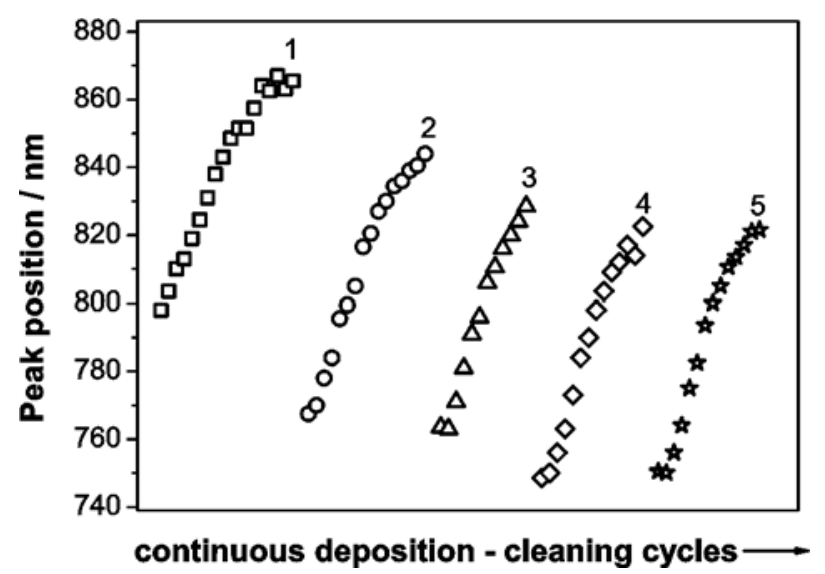

Figure 5.3.30. Peak position as a function of the deposition step, for five consecutive deposition-cleaning cycles. Each symbol (squares, circles, etc.) corresponds to the peak wavelength of the plasmonic resonance after addition of one polyelectrolyte monolayer. Polyelectrolyte layers were added until no further shift in the resonances was visible. Then, the polymeric layers were removed by cleaning the surfaces with a tissue soaked in ethanol. The obtained cleaned surface was used for another deposition cycle (indicated by the change of symbols). The first point in each sequence corresponds to the resonance position after cleaning

After the first cleaning cycles, a blue shift of the resonances of the plain substrate was detected (see initial points of the cycles in Figure 5.3.30). However, no changes in total extinction or sensitivity towards polyelectrolyte binding were apparent for five consecutive depositioncleaning cycles and the shapes of the resonance spectra remained nearly unaffected by the cleaning procedure. The origin of the blue shift of the plasmonic resonance after cleaning is yet not completely clear. It was especially pronounced after the first cleaning step and then decreased until it reached stable values after the fourth cleaning cycle. Probably, two factors are responsible for these phenomena. First, changes in the silicon dioxide layer can cause a blue shift. The sample was prepared using a thermal evaporation process of silicon dioxide. This is known to result in rather poor quality films. ${ }^{[283]}$ Traces of the silicon monoxide precursor or silicon dioxide crystallites that are not fully connected to the matrix might be washed out during the cleaning processes. This would alter the local environment of the particles and lead to a blue shift of the plasmon resonances. The saturation of the shift to a stable wavelength of $750 \mathrm{~nm}$ for the plain nanoparticles after three cleaning cycles supports this assumption. Second, solvent annealing, known to cause blue shifts in the resonance can at least partly be responsible for the shift detected in the first measurement cycles. ${ }^{[122]}$ The blue shift may be avoided by the use of a higher quality silicon dioxide matrix (e.g. by a sputtering process) or an initial annealing step using the polyelectrolyte-free solutions. 


\subsection{Conclusion}

A novel approach to create highly stable nanoparticle arrays was proposed. Shifts in the LSPR maximum upon polyelectrolyte deposition showed a sensitivity of the platform for changes in the refractive index of the local environment of the particles. Long term measurements confirmed the stability of the sensor platforms in buffer solution over extended periods of time. The samples could be cleaned mechanically and consecutive cleaning-deposition cycles did not show any loss in signal intensity or sensitivity, demonstrating the re-usability of the nanoparticles-based sensing platform. The inherent ultra-low surface roughness of the architecture will have a great advantage for the study of binding events in bio-oriented sensing applications, e.g. when using membrane structures assembled on the substrate as sensing matrix, where the membrane formation is crucially influenced by surface roughness. ${ }^{[284]}$

\subsubsection{Nanoscale patterning of solid supported membranes by integrated diffusion barriers}

\subsection{Introduction}

Lipid bilayer membranes that are self-assembled from phospholipid molecules have long been used as model systems to mimic processes occurring in biological membranes. ${ }^{[285-287]}$

They aim at significantly reducing the complexity of the biological system while preserving the key characteristics, especially lateral fluidity and sealing properties between intra- and extracellular space. Among various membrane architectures, tethered lipid bilayer membranes have been recognized as a powerful platform in biomimetics ${ }^{[288]}$ and for the construction of biosensors. ${ }^{[289]}$ Using thiole-terminated anchor lipids with an oligoethylene glycol spacer, such membranes can be assembled onto gold surfaces. ${ }^{[270,290]}$ The proximity of the substrate provides mechanical stability necessary for long term applications, e.g. in sensing devices. Additionally, the spacer part integrated in the anchor lipids is sufficiently long to decouple the bilayer membrane from the substrate and provides a water reservoir between membrane and substrate. As a consequence, membrane proteins, especially channels that span the membrane and protrude into extra- and intracellular space can be incorporated into such architectures since direct contact with the gold surface - leading to protein denaturation - is circumvented. As 
channel proteins are highly sensitive to ions present in the solution, sensing devices have been realized using tethered bilayer membranes. ${ }^{[272,289]}$

For a variety of applications, for example the creation of protein arrays in more sophisticated sensing devices, the control of diffusion in the membrane is a key requirement. The introduction of diffusion barriers - mostly created by photolithography - has been demonstrated to achieve a compartmentalization of the membrane. ${ }^{[291-294]}$

In this chapter, a novel approach for the patterning of tethered bilayer membranes is introduced. Laterally patterned, ultraflat substrates are used to assemble photopolymerizable lipids selectively on the gold parts of the surface. After polymerization and bilayer membrane assembly, a patterned membrane architecture is at hand that features a fluid distal leaflet and nanoscale diffusion barriers incorporated into the proximal leaflet. Compared to existing membrane patterning approaches, this process offers several advantages. First, no photolithographic step is required during the substrate patterning. Thus, the resolution of the diffusion barriers is not limited by the diffraction limit of light and structure sizes down to $30 \mathrm{~nm}$ are created with ease. Furthermore, the immobile fractions are formed by polymerizable lipids and are integrated into the membrane (Figure 5.3.31). They therefore provide stability to the system and allow to circumvent issues related to the undefined lipid bilayer formation at the edges of membrane patches. ${ }^{[295]}$ Additonally, the low roughness of the substrates guarantees the formation of well ordered, highly sealing membranes. ${ }^{[296]}$

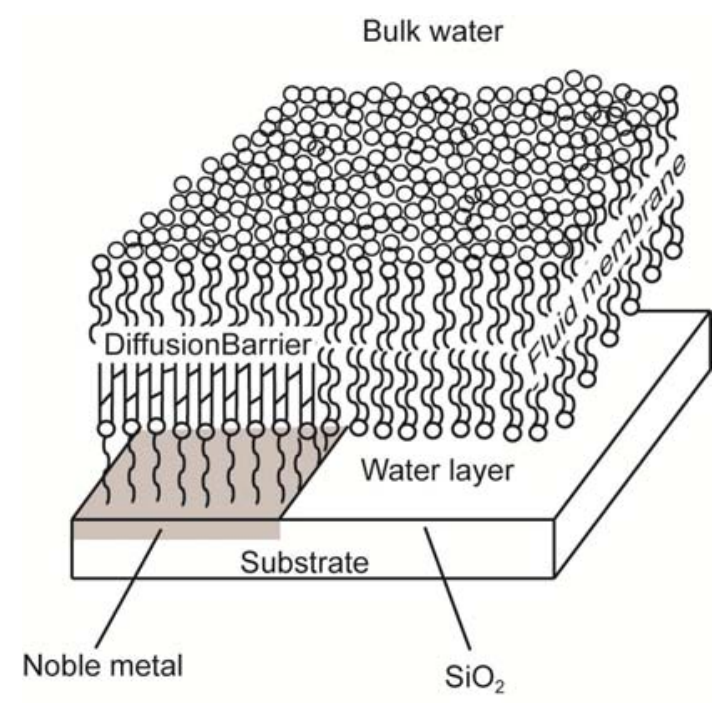

Figure 5.3.31. Schematic illustration of a supported membrane corraled by a self-assembled polymerized lipid barrier. The patterned substrate allows for the controlled assembly of the diffusion barriers embedded into a fluid lipid bilayer membrane. The cartoon was kindly provided by Dr. M. Jung. ${ }^{[263]}$ 


\subsection{Results and Discussion}

\section{Lipid assembly on ultraflat substrates}

The substrates were prepared according to Figure 5.3.26 using differently sized colloids ranging from $180 \mathrm{~nm}$ to $850 \mathrm{~nm}$. An AFM image of a pristine surface after mechanical cleavage prepared from $550 \mathrm{~nm}$ colloids as mask is shown in Figure 5.3.32a. The linescan along the white line presented below (Figure 5.3.32c) highlights the low surface roughness of the substrate. It has been shown that a low surface roughness is essential for the assembly of high quality model membranes with good sealing properties. ${ }^{[296]}$ The polymerizable thio-lipids (structure shown in Figure 5.3.32g), synthesized by Dr. Mathieu Jung ${ }^{[263]}$, were self-assembled on the gold parts of the structured substrate by immersion of the sample in a $0.2 \mathrm{mg} \cdot \mathrm{ml}^{-1}$ ethanolic solution of the lipid for $24 \mathrm{~h}$, followed by thorough rinsing with ethanol. Figure 5.3.32d shows an AFM image of the surface after anchor-lipid functionalization. An increase in height can be seen. The linescan shown in Figure 5.3.32f was used to determine the height of the monolayer to roughly 50 Ångstrom. This value is in good agreement with the calculated persistence length of the anchor lipid (60 A). The AFM phase image of the same sample (Figure 5.3.32e) shows a clear contrast arising from the drastic difference in softness of the lipid molecules compared to the silicon dioxide substrate.

The oxide surfaces of the substrate remained clean from lipids or lipid aggregates. However, the triangular shapes of the gold nanostructures featured less sharp contours after the lipid selfassembly due to the apparition of satellites of few nanometers in the vicinity of the main structures. These satellites resulted from the functionalization of small gold residues, deposited next to the main structures due to diffusion processes occurring during the metal deposition process. A careful examination of the phase image of the pristine substrate (Figure 5.3.32b) reveals the presence of similar satellites. Hence, it can be stated that the surface functionalization process is highly specific and that nanoscale barriers can be assembled selectively on the gold parts of the substrate. 
a)

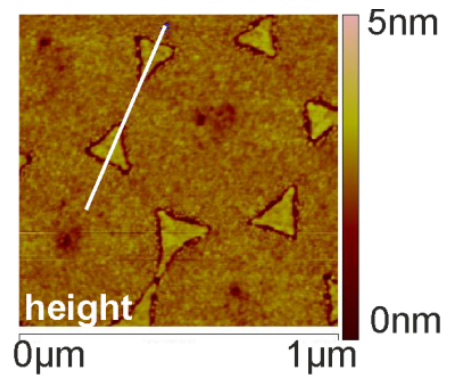

d)

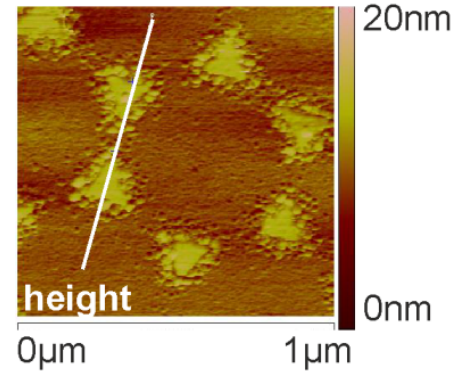

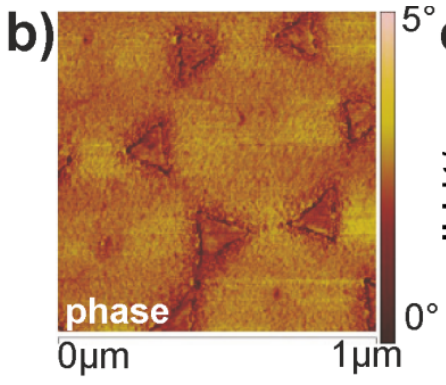
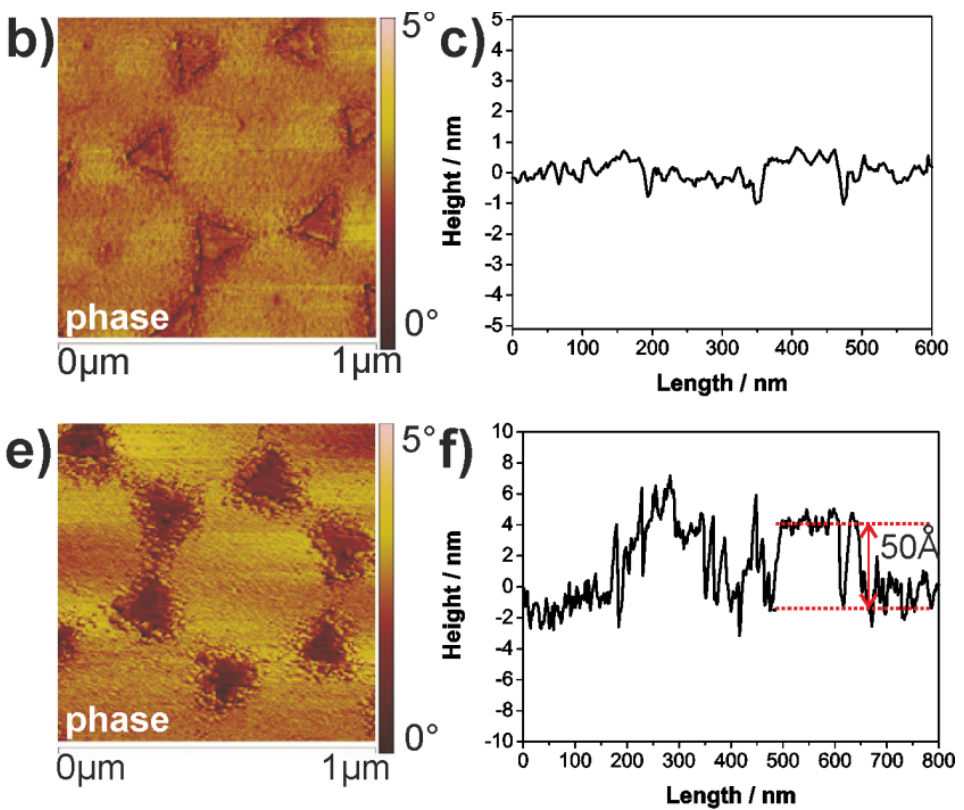

g)
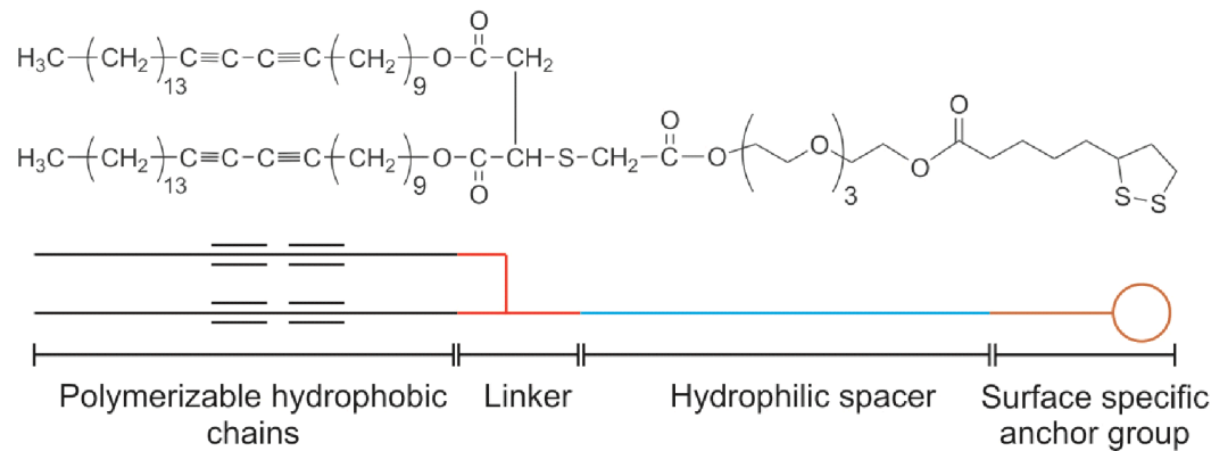

Figure 5.3.32. Functionalization of the embedded gold nanoparticles with polymerizable thiolipids. a-c) AFM investigation of the pristine surface: a) height image; b) phase image; c) line scan along the white line shown in a). d-f) AFM investigation of the substrate after assembly and polymerization of the lipids:

a) height image; b) phase image; c) line scan along the white line shown in c). g) Structure of the polymerizable lipids and schematic description of the different parts of the molecule.

\section{Control of the fluidity of the patterned membrane architecture}

Next, the functionalized substrates were used to create patterned lipid bilayer architectures. First, the substrates were polymerized by UV light to ensure impenetrability and the bilayer was completed by the fusion of small unilamellar vesicles composed of 1,2-diphytanoyl-sn-glycero3-phosphocholine (DPhyPC) and the fluorescently labeled lipid 1,2-dioleoyl-sn-glycero-3phosphoethanolamine-N-(7-nitro-2-1,3-benzoxadiazol-4-yl) (DOPE-NBD). In order to probe the fluidity of the obtained bilayers, the lateral diffusion of the lipids was measured by fluorescence recovery after photobleaching (FRAP) (Figure 5.3.33). The recovery was measured over time and fitted to the model developed by Soumpasis ${ }^{[297]}$ (details are given in the experimental chapter). 

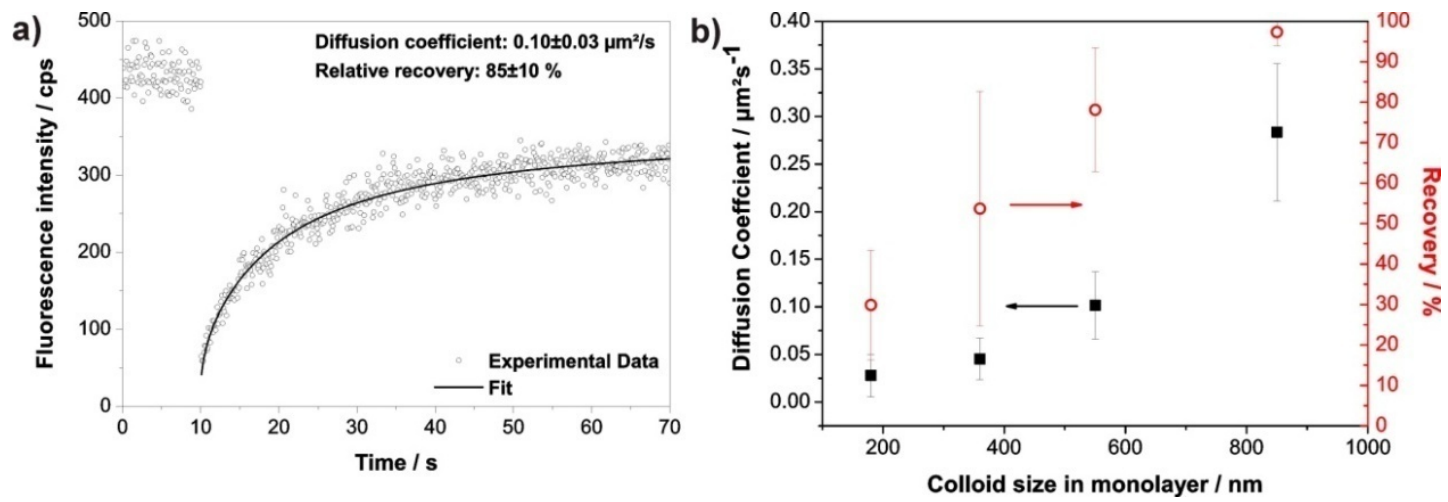

Figure 5.3.33. Fluorescence recovery after photobleaching (FRAP) measurements of the patterned lipid bilayer mebranes. a) Fluorescence recovery with time and fit to termine diffusion coefficient and relative covery exemplarily shown for a substrate patterned with $550 \mathrm{~nm}$ colloids. b) Dependence of diffusion coefficient (black points) and relative recovery (red points) on the size of the colloids used for the creation of the barriers.

Figure 5.3.33a exemplarily shows the fluorescence recovery with time as recorded for the substrate patterned with $550 \mathrm{~nm}$ colloids. As evident from the AFM images (Figure 5.3.32), the nano-barriers were not interconnected so that diffusion of the lipids was possible both in the proximal and distal leaflet of the membrane. The high relative recovery of the fluorescence signal to $85 \%$ of its initial value supports this finding and corroborates with the schematic model of the bilayer membrane presented in Figure 5.3.31. An ordered, fluid bilayer was formed and the diffusion barriers were incorporated into this architecture. The measured diffusion coefficient of $0.10 \pm 0.03 \mu \mathrm{m}^{2} / \mathrm{s}$ was significantly lower than the typical diffusion coefficients of $1-2 \mu \mathrm{m}^{2} / \mathrm{s}$ reported for solid supported membranes. ${ }^{[263,298]}$ This shows that the immobile obstacles had a significant impact on the membrane fluidity. The incomplete recovery is hypothesized to be caused by defects in the monolayer. Such defects, mostly consisting of line defects in the monolayer crystal are transformed into extended gold barriers during the evaporation. Such barriers naturally block the diffusion of lipids in the proximal leaflet and impede a complete recovery of the signal.

The hybrid membrane architecture features barriers in the nanometer regime. Since the size of the diffusion barriers is not limited by the diffraction limit of light, as for the conventional patterning methods, biologically relevant obstacle sizes can be reached, e.g. lipid rafts domains, immobilized protein domains on the cytoskeleton. ${ }^{[298-299]}$

To explore effects of the barrier size and obstacle lattice on the diffusion within the membrane, the size of the colloids used in the substrate patterning process was varied. As the applied patterning procedure yields arrays of triangles corresponding to the voids in the colloidal monolayer, the dimensions of the triangular structures can be precisely adjusted by the used 
colloids. By using colloids of approximately 180, 360, 550 and $850 \mathrm{~nm}$, the immobile obstacles were adjusted to be in between 30 and $250 \mathrm{~nm}$. Due to the specific technique of nanosphere lithography, the fraction of immobile obstacles was intrinsically fixed to $9.3 \%$ (reflecting the surface coverage of voids in a perfectly ordered monolayer).

Figure 5.3.34b shows the dependence of the diffusion coefficients and percentage of fluorescence recovery of the artificial membranes in function of the colloid size used for patterning. The fluidity of the membrane, both in terms of diffusion coefficients and fluorescence recovery, decreased with decreasing barrier size. This behavior is attributed to an increase in the number of obstacles per area the lipids have to pass in their diffusion path.

By varying the barrier size, it was possible to adjust the mobility of the lipids over one order of magnitude from $0.03 \pm 0.02 \mu \mathrm{m}^{2} \cdot \mathrm{s}^{-1}$ to $0.28 \pm 0.07 \mu \mathrm{m}^{2} \cdot \mathrm{s}^{-1}$. These diffusion coefficients reflect that the membranes with the embedded obstacles had a similar fluidity compared to biological cell membranes. ${ }^{[298]}$

The decrease in the size of the colloids led to a decrease in the recovery. This is related to the defect structure of the different monolayers. The amount of defects per area will increase for smaller colloids, as the size of single crystalline domains will be reduced - even assuming perfectly similar order in the individual monolayers - simply due to the decrease in size of the individual colloids. As stated above, defects in the monolayer usually translate into more extended gold areas that act as impenetrable diffusion barriers. Hence, qualitatively, this simple argument explains the detected dependence of the relative recovery. However, one should keep in mind that there might be a threshold barrier size upon which the lipids are blocked completely from diffusing into the bleached spot, thus reducing the amount of fluorescence recovery.

\section{Isolated free lipid corrals surrounded by diffusion barriers}

The nano-structuration with periodically patterned, open structures might be of interest for basic research aiming at a more precise mimic of biological membranes to study e.g. the diffusion behavior of lipids (as demonstrated) or membrane proteins. Of higher technological importance however, is the confinement of molecules to a defined area; e.g. the electrode of a biosensor device that uses membrane proteins to detect binding events of specific molecules in the water phase. Confinement of the sensing unit to a defined place might thus open the door to create more stable devices (as the unit cannot diffuse away from the electrode), high sensitivity for applications e.g. in single protein sensing (using nanoscale electrodes with one protein confined) or to high throughput arrays of sensors. 
a)

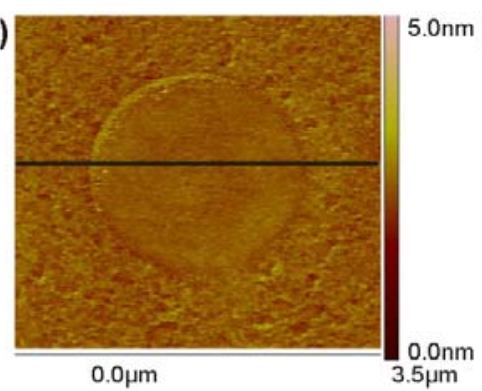

d)

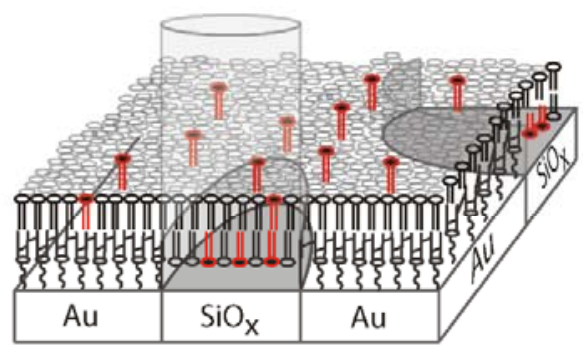

f)

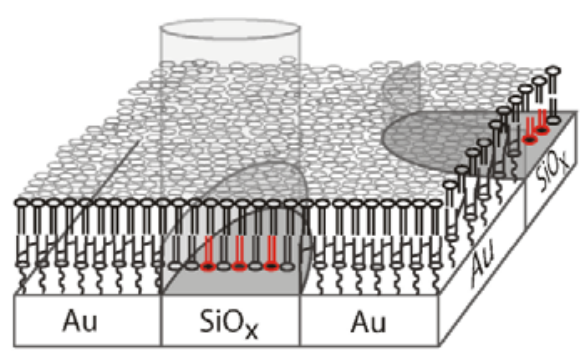

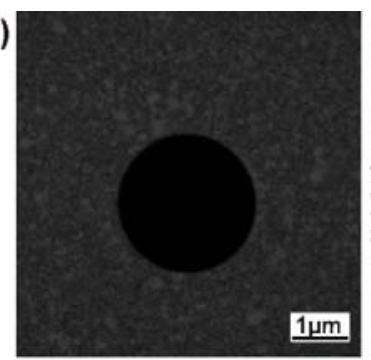

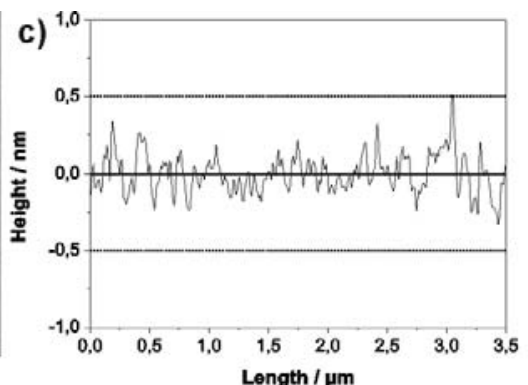

e)

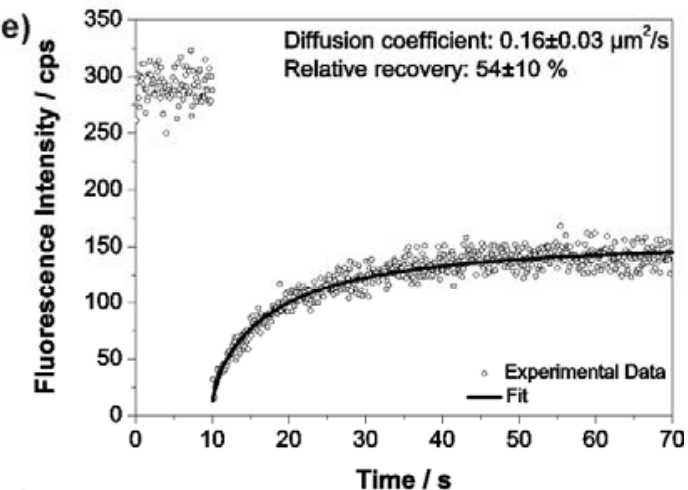

g)

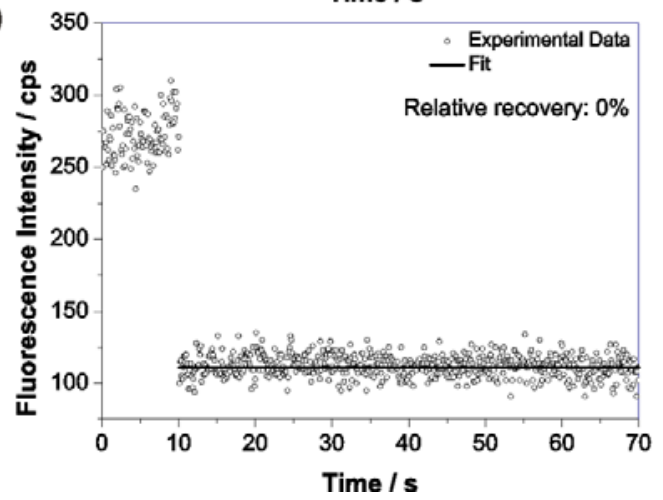

Figure 5.3.34. Characterization of the substrates used for the diffusion barrier experiments. a) AFM height image of a single silicon dioxide disc embedded in a continuous gold film. b) SEM image. c) Linescan along the line shown in a). $d-g$ ) Schematic depiction $(d, f)$ and measured diffusion curves of FRAP experiments $(e, g)$ performed on isolated lipid corrals. d,e) FRAP experiments to probe diffusion in both leaflets simultaneously. f,g) FRAP experiment on the proximal leaflet only.

To demonstrate the possibility to corral lipids in the proximal leaflet of the membrane by polymerized barriers, substrates with isolated silicon dioxide disks embedded in a gold film were prepared in analogy to Figure 5.3.26 but with sparsely distributed, single colloids (glass beads with a size of $2.5 \pm 0.5 \mu \mathrm{m}$ ) as mask. The obtained structures were thus of about the same size as the laser spot used to bleach the lipids in a FRAP experiment. An AFM investigation of the substrate surface is shown in Figure 5.3.34. Again, the extremely low roughness of the embedded structure is visible and further underlined by the line scan in Figure 5.3.34c. The electron micrograph shown in Figure 5.3.34b clearly visualizes the difference between gold (bright) and silicon dioxide (black). 
The gold parts of the substrate were functionalized with the polymerized lipids by self-assembly and UV polymerization. After fusion of lipid vesicles to complete the hybrid membrane structure, the diffusion of the lipids over the oxide islands was assessed by FRAP. Measurements were performed to investigate the diffusion in both leaflets (Figure 5.3.34d) and in the proximal leaflet only (Figure 5.3.34f). For the latter experiments, the fluorophores in the distal leaflet were chemically bleached by reduction with sodium dithionite. ${ }^{[300]}$ For all experiments, the laser spot was focused onto a single silicon dioxide disk.

Diffusion over both leaflets showed a recovery of approximately 50\% (Figure 5.3.34e). Assuming equal distribution of the fluorescently labeled lipids in both leaflets, this corresponds to an effective prevention of diffusion in the inner leaflet and a closed, fluid outer leaflet spanning both fluid membrane parts and photopolymerized lipids, as sketched in Figure 5.3.34d. After the chemical bleaching of the fluorophores in the outer leaflet, the FRAP experiment probed only the diffusion in the inner leaflet (f). No fluorescence recovery was measured (Figure 5.3.34g), indicating that the diffusion of lipids in the proximal leaflet from neighboring corrals was prevented and the polymerized lipids indeed acted as effective diffusion barriers.

\subsection{Conclusion}

An approach to pattern model membranes is presented, where tethered immobile obstacles are embedded in the lipid bilayer in a controlled way and on a nanometer scale. The architecture offers a number of attractive features. First, the size of the immobile obstacles is not restricted by the diffraction limit. This has been a significant limitation when conventional photolithographic methods have been used for a direct patterning of a photo-polymerizable membrane or for the structuring of substrates with diffusion barriers. By employing nanosphere lithography as a technique to structure the template surface, a precise adjustment of barriers on the nanometer scale is feasible. Second, the tethered lipids are part of the membrane and thus decouple the bilayer from the substrate. At the same time they act as immobile obstacles. The flatness of the patterned substrate allows the barriers to merge homogeneously into the bilayer membrane and avoids problems arising artificial material and incomplete sealing properties of membranes at the edges of topological barriers.

Two distinct configurations of patterns were tested. First, using nanoscale arrays of diffusion barriers as produced by classical nanosphere lithography, the patterned membrane architecture remained fluid and a strong influence of the measured diffusion coefficient with barrier size was detected. This may have possible applications for the fundamental understanding of biological membranes that feature nanoscale diffusion barriers as well. Second, micrometer sized 
individual corrals were prepared using sparsely distributed colloids as mask. FRAP measurements on these spots showed that the resulting membrane remained fluid in the distal leaflet while diffusion in the proximal leaflet was effectively prevented. Such architectures might have a strong impact on next-generation biosensor design as they allow the confinement of the sensing unit to a defined place in the membrane. 


\section{Experimental Section}

\subsection{General procedures}

\subsubsection{Miniemulsion polymerization}

The standard recipe for a direct miniemulsion was applied until otherwise stated.

The monomer phase, consisting of $6 \mathrm{~g}$ of monomer (typically styrene), $250 \mathrm{mg}$ hexadecane, $100 \mathrm{mg}$ V59 (2,2'-azobis(2-methylbutyronitrile)) and varying amounts of metal complex specified in the individual chapters are mixed with the water phase $(24 \mathrm{~g}$ ultrapure water and $60 \mathrm{mg}$ sodium dodecyl sulfate) and stirred at $1800 \mathrm{rpm}$ for $60 \mathrm{~min}$. Miniemulsification is achieved by ultrasonicating the mixture under ice-cooling for $120 \mathrm{~s}$ with a $1 / 2$ " tip at $90 \%$ amplitude following a 10 s-pulse-10 s-break protocol, (Branson digital sonifier 450-D, Dietzenbach, Germany). Afterwards, the mixture is heated to $72{ }^{\circ} \mathrm{C}$ and polymerized for $12 \mathrm{~h}$ under gentle continuous stirring.

After cooling to room temperature, the colloidal dispersion is filtered and extensively dialysed (Visking tubes, MWCO $14.000 \mathrm{~g} \cdot \mathrm{mol}^{-1}$, Carl Roth, Karlsruhe, Germany) until the conductivity of the water phase after dialysis was similar to the value measured for dionized water.

\subsubsection{Surfactant-free emulsion polymerization}

All plain polystyrene latex particles used for the assembly of colloidal monolayers throughout this thesis were prepared by surfactant-free emulsion polymerization following a modified protocol reported from Goodwin. ${ }^{[29]}$

Briefly, water (milliQ grade) was heated to $80^{\circ} \mathrm{C}$ in a reaction flask equipped with a reflux condenser and degassed by bubbling argon through the liquid for $30 \mathrm{~min}$. Monomer was added by a syringe and the mixture was stirred at high speeds ( 1200 rpm) for $10 \mathrm{~min}$. Subsequently, the comonomer (Sodium para-styrene sulfonate (NapSS) or acrylic acid (AA), dissolved in $5 \mathrm{ml}$ of water was added to the mixture. After $5 \mathrm{~min}$, the reaction initiator (ammonium peroxodisulfate; APS), dissolved in $5 \mathrm{ml}$ of water was added by a syringe as well to initiate the emulsion polymerization process. During the reaction, the stirring was changed to 
approximately $700 \mathrm{rpm}$ and a gentle argon stream ( 1 bubble/s) was adjusted. The reaction was stopped after $24 \mathrm{~h}$. After cooling to room temperature, the dispersion was filtered using a regular tea-sieve and dialyzed extensively (Visking tubes, MWCO $14.000 \mathrm{~g} / \mathrm{mol}$, Carl Roth, Karlsruhe, Germany).

An overview over all prepared colloidal samples is given in Table 6.1.1 and Table 6.1.2.

Colloids with a diameter of $850 \mathrm{~nm}$ and $1063 \mathrm{~nm}$ were prepared by a seeded emulsion process and kindly provided by Markus Retsch and Gabrielle Schäfer, respectively.

Table 6.1.1. Colloidal particles prepared by surfactant-free emulsion polymerization with sodium para styrene sulfonate (NapSS) as comonomer.

\begin{tabular}{cccccc}
\hline $\begin{array}{c}\text { Sample } \\
\text { name }\end{array}$ & $\begin{array}{c}\text { Diameter } \\
\text { of colloids } \\
\text { [nm] }\end{array}$ & $\begin{array}{c}\text { Styrene } \\
\text { [g] }\end{array}$ & $\begin{array}{c}\text { Comonomer } \\
\text { NapSS } \\
\text { [mg] }\end{array}$ & $\begin{array}{c}\text { Initiator (APS) } \\
\text { [mg] }\end{array}$ & $\mathbf{H}_{\mathbf{2}} \mathbf{O}$ \\
\hline NV143-25 & 138 & 8 & 30 & 100 & 250 \\
NV143-8 & 172 & 15 & 60 & 100 & 250 \\
NV143-18 & 179 & 15 & 30 & 150 & 250 \\
NV143-19 & 188 & 15 & 20 & 100 & 250 \\
NV143-21 & 223 & 15 & 10 & 100 & 250 \\
NV143-20 & 246 & 15 & 5 & 100 & 250 \\
NV143-22 & 271 & 15 & 10 & 50 & 125 \\
NV143-9 & 326 & 15 & 5 & 100 & 250 \\
NV143-11 & 408 & 30 & 5 & 100 & 300 \\
NV143-1 & 413 & 15 & 0 & 150 & 300 \\
NV143-2 & 476 & 15 & 0 & 100 & 300 \\
\hline
\end{tabular}

Table 6.1.2. Colloidal particles prepared by surfactant-free emulsion polymerization with acrylic acid

\begin{tabular}{cccccc}
\hline $\begin{array}{c}\text { Sample } \\
\text { name }\end{array}$ & $\begin{array}{c}\text { Diameter } \\
\text { of colloids } \\
\text { [nm] }\end{array}$ & $\begin{array}{c}\text { Styrene } \\
\text { [g] }\end{array}$ & $\begin{array}{c}\text { Comonomer } \\
\text { AA } \\
\text { [mg] }\end{array}$ & $\begin{array}{c}\text { Initiator (APS) } \\
\text { [mg] }\end{array}$ & $\mathbf{H}_{2} \mathbf{O}$ \\
\hline NV180-12 & 169 & 1.3 & 100 & 100 & 125 \\
NV180-9 & 174 & 1 & 100 & 100 & 125 \\
NV180-7 & 202 & 1.6 & 100 & 100 & 125 \\
NV180-11 & 225 & 3 & 100 & 100 & 125 \\
NV180-2 & 303 & 5 & 100 & 100 & 125 \\
NV180-13 & 315 & 6 & 100 & 100 & 125 \\
NV180-10 & 336 & 8 & 100 & 100 & 125 \\
NV180-14 & 409 & 7.5 & 100 & 100 & 125 \\
NV180-3 & 415 & 8.5 & 100 & 100 & 125 \\
\hline
\end{tabular}




\begin{tabular}{llllll}
\hline NV180-8 & 422 & 6.5 & 100 & 100 & 125 \\
NV180-1 & 550 & 15 & 200 & 100 & 250 \\
\hline
\end{tabular}

\subsubsection{Treatment of silicon wafer substrates prior use}

All silicon wafers were carefully cleaned before use. The cleaning was performed by a wetchemical oxidation method. The wafers were cut (approximately $2 \times 3 \mathrm{~cm}^{2}$ ) and placed into a Färber Box. $50 \mathrm{ml}$ ultrapure water, $10 \mathrm{ml}$ concentrated ammonia solution (25\%) and $10 \mathrm{ml}$ hydrogen peroxide $(33 \%)$ were added and heated to $75{ }^{\circ} \mathrm{C}$ for $45 \mathrm{~min}$. After cooling, the substrates were extensively rinsed with water and stored in ethanol until use. The treatment oxidizes the silicon wafer surface and induces a water contact angle of almost $0^{\circ}$.

\subsubsection{Nanostructure fabrication}

All evaporations were carried out in a Balzers PLS500 evaporation chamber with a Pfeiffer vacuum pump (Balzers AG, Liechtenstein). The pressure in the evaporation chamber was always below $5 \cdot 10^{-6} \mathrm{mbar}$ before the start of the evaporation process. Gold and chromium were evaporated at a rate of approx. $0.02 \mathrm{~nm} / \mathrm{s}$, silicon monoxide was evaporated at a rate of approx. $0.1 \mathrm{~nm} / \mathrm{s}$ with an oxygen partial pressure of $1 \cdot 10^{-4}$ mbar to form the silicon dioxide matrix. If required, the samples were etched with a reactive ion beam (RR-I SQ76, Roth \& Rau, Wüstenbrand, Germany). As etching gas, $\mathrm{Ar}, \mathrm{CF}_{4}$ or $\mathrm{O}_{2}$ was used. The individual protocols for the different fabrication processes will be described in the experimental section of the individual subchapters.

\subsubsection{Scanning electron microscopy}

All scanning electron micrographs were recorded on a Gemini 1530 microscope (Carl Zeiss AG, Oberkochen, Germany). If not stated otherwise, colloidal dispersions were routinely characterized by adding a drop $(\sim 10 \mu \mathrm{l})$ of a $0.01 \mathrm{wt.}-\%$ dispersion in water to a hydrophilic silicon wafer. The drop was left to evaporate, leaving a film of colloids on the substrate that was placed into the electron microscope. 


\subsubsection{UV/Vis-NIR spectroscopy}

Polarization-dependent UV-visible spectra were recorded on a Perkin Elmer Lambda 900 spectrometer.

\subsubsection{Photographs}

All photographs shown in this thesis were taken with a Canon EOS 450 camera body equipped with a Sigma objective $(17-200 \mathrm{~mm})$.

\subsection{Materials}

Unless otherwise stated, all chemicals were purchased from Sigma Aldrich (Seelze, Germany) and used as received. Styrene and methyl methacrylate were distilled under reduced pressure before used in polymerization reactions to remove the inhibitor. All metal complexes were purchased from STREM chemicals (STREM chemicals inc., Kehl, Germany)

Water of ultra-high purity (milliQ grade; resistivity $=18.2 \mathrm{M} \Omega \cdot \mathrm{cm}$ ) was obtained from a Milli-Q Gradient water purification system (Millipore, Molsheim, France). Silicon wafers, polished on one side were purchased from CrysTec Kristalltechnologie (Berlin, Germany).

\subsection{Experimental details for chapter 5.1.1 (Accurate elemental analysis of metal-containing polymer latexes)}

\subsubsection{Particle synthesis}

Miniemulsion particles were synthesized following the standard protocol and adding $60 \mathrm{mg}$ of the respective metal complexes. To avoid inaccuracies in the measurements due to loss of complex during dialysis, the samples were used as produced. As seeded emulsion particles, 
different samples prepared as described in 5.1.2.5 were used. The hydrodynamic diameter $\mathrm{d}_{\mathrm{H}}$ of the particles was determined using photon cross correlation spectroscopy (PCCS) with the commercial particle sizer Nanophox (Sympatec, Clausthal-Zellerfeld, Germany). For the measurement, the latex dispersions were diluted to approx. $0.1 \mathrm{wt} . \mathrm{-} \%$ with ultrapure water. As average particle size, the $\mathrm{x}_{50}$-value ( $50 \%$ of the particle diameters below this value) of the intensity weighted particle size distribution was used.

\subsubsection{Zeta potential measurements}

Zeta potentials were measured using a Zetasizer 3000 HSA (Malvern Instruments $\mathrm{GmbH}$, Herrenberg, Germany). To determine the zeta potential, particle dispersions with 0.01 wt.-\% were used. Electrolyte was added for the measurements as stated in Table 4. Five measurements were averaged for the zeta potential given in this article.

The solid content of the latex dispersion was determined gravimetrically by drying the dispersion in vacuum at $50^{\circ} \mathrm{C}$ for $24 \mathrm{~h}$.

\subsubsection{Inductively coupled plasma - optical emission spectrometry (ICP-OES) measurements}

All measurements were performed with an ACTIVA M spectrometer (Horiba Jobin Yvon, Bernsheim, Germany) equipped with a meinhardt-type nebulizer, a cyclone chamber and controlled by ACTIVAnalyst 5.4 software.

The following conditions were applied unless stated otherwise: $1250 \mathrm{~W}$ forward plasma power, $12 \mathrm{~L} \cdot \mathrm{min}^{-1}$ Ar flow and $15 \mathrm{rpm}$ pump flow. The colloidal dispersions measured were diluted with water to $0.1 \mathrm{wt}-\%$ of colloids and sonicated for 3 minutes prior to measuring The Argon emission line at $404.442 \mathrm{~nm}$ was used as reference line. Measurements were performed using 4 different standard concentrations, at least 3 different elemental emission lines and $5 \mathrm{~s}$ integration time. As baseline correction, a dynamic underground correction provided by the software was used.

The emission lines chosen for the characterization of the respective elements were:

Pt: $212.863 \mathrm{~nm} ; 217.469 \mathrm{~nm} ; 224.552 \mathrm{~nm} ; 299.797 \mathrm{~nm}$

Fe: $248.816 \mathrm{~nm} ; 258.588 \mathrm{~nm} ; 358.119 \mathrm{~nm}$

Eu: $272.778 \mathrm{~nm} ; 372.595 \mathrm{~nm} ; 381.967 \mathrm{~nm}$ 


\subsubsection{Surface tension measurements}

Surface tension measurements were performed on a DCAT21 tensiometer (Dataphysics, Fildestadt, Germany) using a DuNoüy ring with a diameter of $4.85 \mathrm{~mm}$ and a wire diameter of $0.15 \mathrm{~mm}$. The motor speed during measurement was set to $0.5 \mathrm{~mm} \cdot \mathrm{s}^{-1}$. The samples used for measuring were the identical to those used for ICP-OES analysis and consisted of $0.1 \mathrm{wt} .-\%$ dispersions of colloids with varying amounts of SDS. For comparison, similar solutions with equal amounts of SDS without colloids were used.

All values presented were averaged over 10 repetitions of the surface tension measurements.

After each sample, the ring was extensively rinsed with water, ethanol and heated in a propane flame.

\subsection{Experimental details for chapter 5.1.2 (Synthesis of metal-complex containing latex particles)}

\subsubsection{Platinum-complex containing polymer particles by emulsion polymerization}

Platinum acetylacetonate containing latex particles were produced by surfactant free emulsion polymerization, following a similar protocol as for the plain polystyrene particles described above. Table 6.4.1 shows the experimental details. The first entry ( $4 \mathrm{~g}$ styrene) was used to prepare the particles used in the seeded emulsion polymerization reactions of chapter 5.1.2.5.7; the following three entries are shown in chapter 5.1.2.2.1 for the studies on the incorporation of platinum(II)acetylacetonate in varying amounts.

Table 6.4.1. Surfactant free emulsion polymerization in the presence of platinum(II)acetylacetonate.

\begin{tabular}{|c|c|c|c|c|c|}
\hline Styrene & $\begin{array}{c}\text { Comonomer } \\
\text { (NapSS) }\end{array}$ & Initiator (APS) & $\mathrm{H}_{2} \mathrm{O}$ & $\overline{P t(a c a c)_{2}}$ & Pt(acac)2 \\
\hline$/ g$ & $/ \mathrm{mg}$ & $/ \mathrm{mg}$ & $/ \mathbf{m l}$ & $/ \mathbf{m g}$ & /wt.-\% of monomer \\
\hline 4 & 35 & 75 & 125 & 160 & 4 \\
\hline 7.5 & 15 & 50 & 125 & 75 & 1 \\
\hline 7.5 & 15 & 50 & 125 & 150 & 2 \\
\hline
\end{tabular}




\begin{tabular}{llllll}
\hline 7.5 & 15 & 50 & 125 & 300 & 4
\end{tabular}

\subsubsection{Spinning drop tensiometry measurements to investigate the interfacial behavior of the metal-ß-diketonate complexes}

Spinning drop tensiometry (SDT) was performed using an SVT20 spinning drop tensiometer (Dataphysics, Filderstadt, Germany). The technique measures the interfacial tension between two immiscible phases by analysis of the dimensions and shape of a droplet of one lighter phase centered in a second, continuous and denser phase in a rotating capillary tube. ${ }^{[172]}$ Toluene was chosen as the lighter, organic phase because most $\beta$-diketonate complexes dissolve in it in high concentrations. All measurements were performed using UHP water. Typically, a droplet of 50 $\mu l$ of toluene with the respective metal complex was injected into the water-filled measurement capillary using a high precision syringe (Hamilton Bonaduz AG, Switzerland). Interfacial tensions were determined at $22{ }^{\circ} \mathrm{C}$ with a spinning rate of $10000 \mathrm{~min}^{-1}$ by video analysis of the cylindrical droplet. From the cylindrical profile, the interfacial tension between two phases 1 and 2 can be directly calculated analytically using Vonnegut's equation: ${ }^{[173]}$

$$
\gamma_{1,2}=\frac{1}{4}\left(\rho_{2}-\rho_{1}\right) \omega^{2} R_{1}^{3}
$$

With a cyclindrical shape, the interfacial tension $\gamma_{1,2}$ only depends on the difference in density of continuous $\left(\rho_{2}\right)$ and dispersed phase $\left(\rho_{1}\right)$, the rotational speed $\omega$ and the radius $R_{1}$ of the cylinder formed by phase 1 in phase 2 .

Complex concentrations of 10 and, solubility permitting, $50 \mathrm{mmol} \cdot \mathrm{kg}^{-1}$ were chosen, corresponding to $c a 0.4$ and 2 wt.- $\%$ of metal complex, respectively. To minimize contaminations, the glass capillary was cleaned consecutively with ethanol (5 times), water (5 times) and ethanol (1 time) and dried with nitrogen immediately before use.

\subsubsection{Miniemulsion polymerization containing $\mathrm{Fe}(\mathrm{acac})_{3}$ and $\mathrm{Pt}(\mathrm{acac})_{2}$}

All miniemulsion polymerizations were performed according to the standard recipe with varying amounts of metal-complexes and surfactant SDS. Table 6.4.2 presents the reaction details. Figure 5.1.15 of chapter 5.1.2.4.1 presents the results of the ICP-OES analysis for 
particles prepared with $60 \mathrm{mg}$ and $300 \mathrm{mg}$ of SDS, respectively. The complete set of ICP-OES measurements for all surfactant concentrations applied is shown in Figure 6.4.1.

Table 6.4.2. Miniemulsion polymerization in the presence of $\mathrm{Fe}(\mathrm{acac})_{3}$ and $\mathrm{Pt}(\mathrm{acac})_{2}$

\begin{tabular}{|c|c|c|c|c|c|c|}
\hline $\begin{array}{l}\text { Styrene } \\
\text { /mg }\end{array}$ & 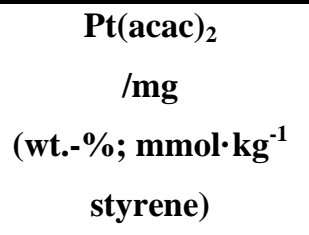 & $\begin{array}{c}\text { Fe(acac) })_{3} \\
/ \mathrm{mg} \\
\left(\text { wt.-\%; } \mathrm{mmol} \cdot \mathrm{kg}^{-1}\right. \\
\text { styrene) }\end{array}$ & $\begin{array}{c}\text { SDS } \\
\text { /mg } \\
\text { (wt.-\% } \\
\text { styrene) }\end{array}$ & $\begin{array}{c}\text { Hexa- } \\
\text { decane } \\
\text { /mg }\end{array}$ & $/ \mathbf{m g}$ & $\mathrm{H}_{2} \mathrm{O}$ \\
\hline 6000 & $30(0.5 ; 12.6)$ & $26.9(0.45 ; 12.6)$ & 30 & 250 & 100 & 24 \\
\hline 6000 & $60(1.0 ; 25.2)$ & $53.9(0.90 ; 25.2)$ & 30 & 250 & 100 & 24 \\
\hline 6000 & $120(2.0 ; 50.4)$ & $107.8(1.80 ; 50.4)$ & 30 & 250 & 100 & 24 \\
\hline 6000 & $180(3.0 ; 76.6)$ & $161.6(2.69 ; 76.6)$ & 30 & 250 & 100 & 24 \\
\hline 6000 & $30(0.5 ; 12.6)$ & $26.9(0.45 ; 12.6)$ & 60 & 250 & 100 & 24 \\
\hline 6000 & $60(1.0 ; 25.2)$ & $53.9(0.90 ; 25.2)$ & 60 & 250 & 100 & 24 \\
\hline 6000 & $120(2.0 ; 50.4)$ & $107.8(1.80 ; 50.4)$ & 60 & 250 & 100 & 24 \\
\hline 6000 & $180(3.0 ; 76.6)$ & $161.6(2.69 ; 76.6)$ & 60 & 250 & 100 & 24 \\
\hline 6000 & $30(0.5 ; 12.6)$ & $26.9(0.45 ; 12.6)$ & 120 & 250 & 100 & 24 \\
\hline 6000 & $60(1.0 ; 25.2)$ & $53.9(0.90 ; 25.2)$ & 120 & 250 & 100 & 24 \\
\hline 6000 & $120(2.0 ; 50.4)$ & $107.8(1.80 ; 50.4)$ & 120 & 250 & 100 & 24 \\
\hline 6000 & $180(3.0 ; 76.6)$ & $161.6(2.69 ; 76.6)$ & 120 & 250 & 100 & 24 \\
\hline 6000 & $30(0.5 ; 12.6)$ & $26.9(0.45 ; 12.6)$ & 300 & 250 & 100 & 24 \\
\hline 6000 & $60(1.0 ; 25.2)$ & $53.9(0.90 ; 25.2)$ & 300 & 250 & 100 & 24 \\
\hline 6000 & $120(2.0 ; 50.4)$ & $107.8(1.80 ; 50.4)$ & 300 & 250 & 100 & 24 \\
\hline 6000 & $180(3.0 ; 76.6)$ & $161.6(2.69 ; 76.6)$ & 300 & 250 & 100 & 24 \\
\hline
\end{tabular}

For the ICP-OES examination of the mechanism, the emulsions were produced according to the standard recipe with $60 \mathrm{mg}$ SDS and $1.0 \mathrm{wt} .-\% \mathrm{Pt}(\mathrm{acac})_{2}\left(25.2 \mathrm{mmol} \cdot \mathrm{kg}^{-1}\right)$ and $0.90 \mathrm{wt} .-\%$ $\mathrm{Fe}(\mathrm{acac})_{3}\left(25.2 \mathrm{mmol} \cdot \mathrm{kg}^{-1}\right)$.

$10 \mathrm{~g}$ of the emulsions were taken after sonication but before polymerization and collapsed with $10 \mathrm{~g}$ of a $1 \mathrm{~mol} \cdot \mathrm{l}^{-1} \mathrm{CaCl}_{2}$ solution. The water phase was separated and analyzed. $10 \mathrm{~g}$ of the dispersions were taken and collapsed following a similar protocol. ICP-OES analysis was performed with matrix matched standards. The remaining $10 \mathrm{~g}$ of the dispersions were extensive dialysed as described in the chapter. 

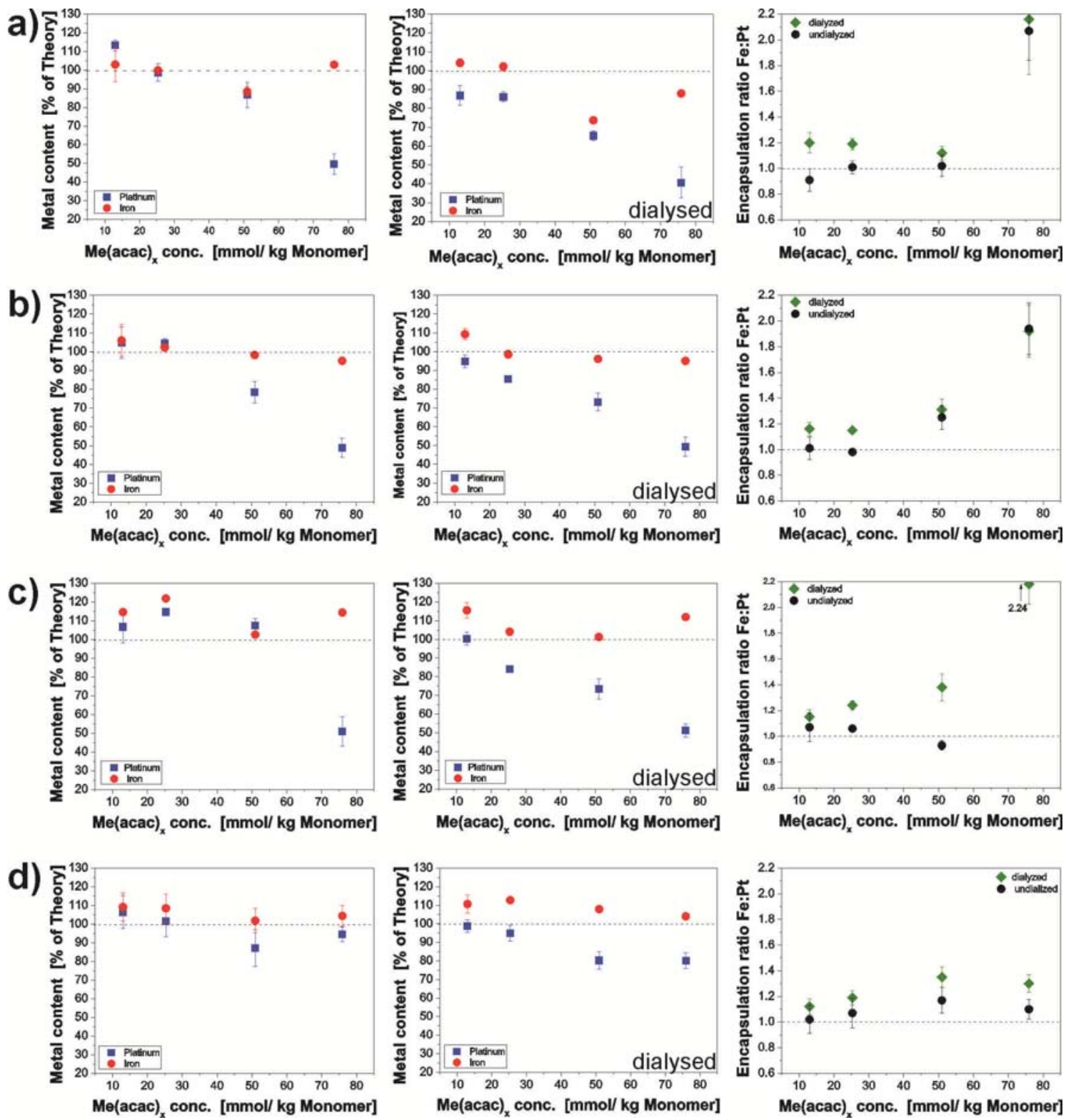

Figure 6.4.1 Complete set of ICP-OES measurements for polystyrene samples containing varying amounts of iron(III)acetylacetonate and platinum(II)acetylacetonate with different amounts of SDS as surfactant. a) 0.5 wt.- $\%$ SDS; b) 1 wt.- $\%$ SDS (as shown in the results and discussion chapter), c) 2 wt.- $\%$

SDS; d) 5 wt.- $\%$ SDS (as shown in the results and discussion chapter)

\subsubsection{Seeded emulsion polymerization}

The protocol given here can be considered the standard protocol. All reactions deviating from this protocol are specified in the main text.

A dispersion of $0.1 \mathrm{wt} .-\%$ of seed particles with $0.01 \mathrm{wt.} . \%$ of SDS (relative to the amount of water used) was heated to $75^{\circ} \mathrm{C}$. Ammonium peroxodisulfate, (APS, $\left.\left(\mathrm{NH}_{4}\right)_{2} \mathrm{~S}_{2} \mathrm{O}_{8}\right), 10-15 \%$ of the monomer added was dissolved in a small amount of ultra-pure water and added to the dispersion. Styrene as monomer was added to the solution using a syringe pump with a flow rate 
of $1 \mathrm{ml} \cdot \mathrm{h}^{-1}$. The amount of monomer was varied to obtain different sizes of the resulting seeded particles. The reaction was stirred for $24 \mathrm{~h}$ at $80^{\circ} \mathrm{C}$ under argon atmosphere. After completion, the final dispersion was dialyzed extensively.

\subsection{Experimental details for chapter 5.2 (Assembly of colloidal monolayer architectures)}

\subsubsection{Convenient production of close-packed monolayers using direct assembly at the air/water interface}

\subsubsection{Monolayer crystallization procedure}

The aqueous colloidal suspensions (typically $\sim 5 \mathrm{wt} .-\%$ ) were diluted with ethanol to form a 50 vol.-\% mixture of dispersion and ethanol and applied to the water surface via a partially immersed hydrophilic glass slide with at a tilt angle of approx. $45^{\circ}$ with respect to the water surface following a procedure adapted from Weekes et al. ${ }^{[72]}$ To help the direct crystallization process, small amounts of SDS were added to the water phase prior spreading of the colloids. The monolayer readily formed at the air/water interface as could be seen by the bright, colorful Bragg reflexes. A hydrophilic substrate (typically a silicon wafer) was immersed into the subphase and elevated under a shallow angle to transfer the monolayer. The substrate was dried under an angle of approximately $45^{\circ}$.

\subsubsection{Non-close packed monolayers by plasma etching}

\subsubsection{Plasma induced size reduction}

All process parameters are specified in the main text. Generally, the etching reactions were carried out in a commercially available plasma cleaner (Model femto, Diener electronic, Nagold, Germany). 
After placing the samples into the plasma chamber, the chamber was evacuated and flushed with the gas used for the etching experiment for three consecutive cycles. The temperature of the bottom plate of the plasma chamber was controlled by a cryostat with a water/ethylene glycol mixture.

\subsubsection{Nanostructure fabrication}

All evaporations were carried out in a Balzers PLS500 evaporation chamber with a Pfeiffer vacuum pump (Balzers AG, Liechtenstein). The samples were etched with a reactive ion beam (RR-I SQ76, Roth \& Rau, Wüstenbrand, Germany) normal to the surface of the substrate. As etching gas, a mixture of $\mathrm{Ar}, \mathrm{CF}_{4}$ and $\mathrm{O}_{2}$ was used. After the etching procedure, the colloids were removed using elastomeric glue (Marabu Fixogum, Tamm, Germany) that was applied onto the sample surface, hardened for $12 \mathrm{~h}$ at room temperature and then removed mechanically.

\subsubsection{Ordered binary monolayers with adjustable stoichiometries}

\subsubsection{Particle charge detection}

The density of phosphonate groups on the particle surface was determined by titration against the opposite charged polyelectrolyte poly(diallyldimethyl ammoniumchloride) (PDADMAC) using a particle charge detector (Mütek $\mathrm{GmbH}$, Germany) in combination with a Titrino Automatic Titrator (Metrohm AG, Switzerland). For the measurement, $10 \mathrm{~mL}$ of the latex sample with a solid content of $1 \mathrm{~g} \cdot \mathrm{l}^{-1}$ were used. The amounts of groups were calculated from the amount of consumed polyelectrolyte as demonstrated by Musyanovich et al. ${ }^{[22]}$

\subsubsection{Surface pressure - area isotherms and surface lowering transfer}

The Langmuir trough model KSV5000 (formerly KSV instruments; now KSV NIMA, Espoo, Finland) with a length of $48.8 \mathrm{~cm}$, a width of $15 \mathrm{~cm}$ and an area of $732 \mathrm{~cm}^{2}$ was used for the monolayer preparation. Colloidal dispersions ( $\sim 5 \mathrm{wt} .-\%$ in water) were diluted with ethanol (1:1 
per volume) and added to the trough via a tilted glass slide partially immersed into the subphase at an angle of approximately $45^{\circ}$ to the water surface. ${ }^{[72]}$ After equilibration for $5 \mathrm{~min}$, the surface layer was compressed with a speed of $20 \mathrm{~mm}$ per min. Surface pressure-area isotherms were recorded using paper Wilhelmy plates that were rotated with an angle of approximately $45^{\circ}$ with respect to the barriers. Transfers of the monolayers to solid substrates were performed by lowering the water surface until the substrate surface was covered with colloids as schematically shown in Figure 1. The $\mathrm{pH}$ of the subphase was adjusted with $\mathrm{NaOH}$ solution.

\subsubsection{Statistical evaluation}

Three to four electron microscopy images with a magnification equal to the image shown in Figure 5.2.21i were used for the statistical analysis of each sample. All interstitial sites belonging to a crystalline domain of the monolayer of the large spheres were counted and assigned to one of the categories presented in Table 5.2.4. The number of sites counted varied between 600 and 1000 .

\subsubsection{Interfacial polymerization of butyl cyanacrylate to visualize the contact angle of the particles}

Figure 6.5.1 graphically illustrates the process used to image the position of the colloids at the interface as presented in chapter 5.2.3.2.5. Figure 6.5.1e shows the reaction scheme of the interfacial polymerization. In a closed chamber, a few drops of n-butyl cyanoacrylate are heated to $50{ }^{\circ} \mathrm{C}$. Adjacent to the BCA, a crystallization dish containing a monolayer floating at the air water interface is placed. Monomer diffuses through the vapor phase until it eventually comes into contact with water. Upon contact, the anion is formed that initiates polymerization. As more and more monomer diffuses to the interface, a polymeric layer of poly(n-butyl cyanoacrylate) (PBCA) appears. As the anions are the most hydrophilic compound, they will continue to reside at the polymer-water interface. Thus, the polymeric layer grows into the water phase. Additional monomer diffuses through the polymeric layer towards the reaction locus. After several hours of reaction, the monomer is consumed and the colloidal monolayer embedded into the PBCA is transferred manually to a silicon wafer. In side-view images, the position of the colloids can now be visualized by electron microscopy. 

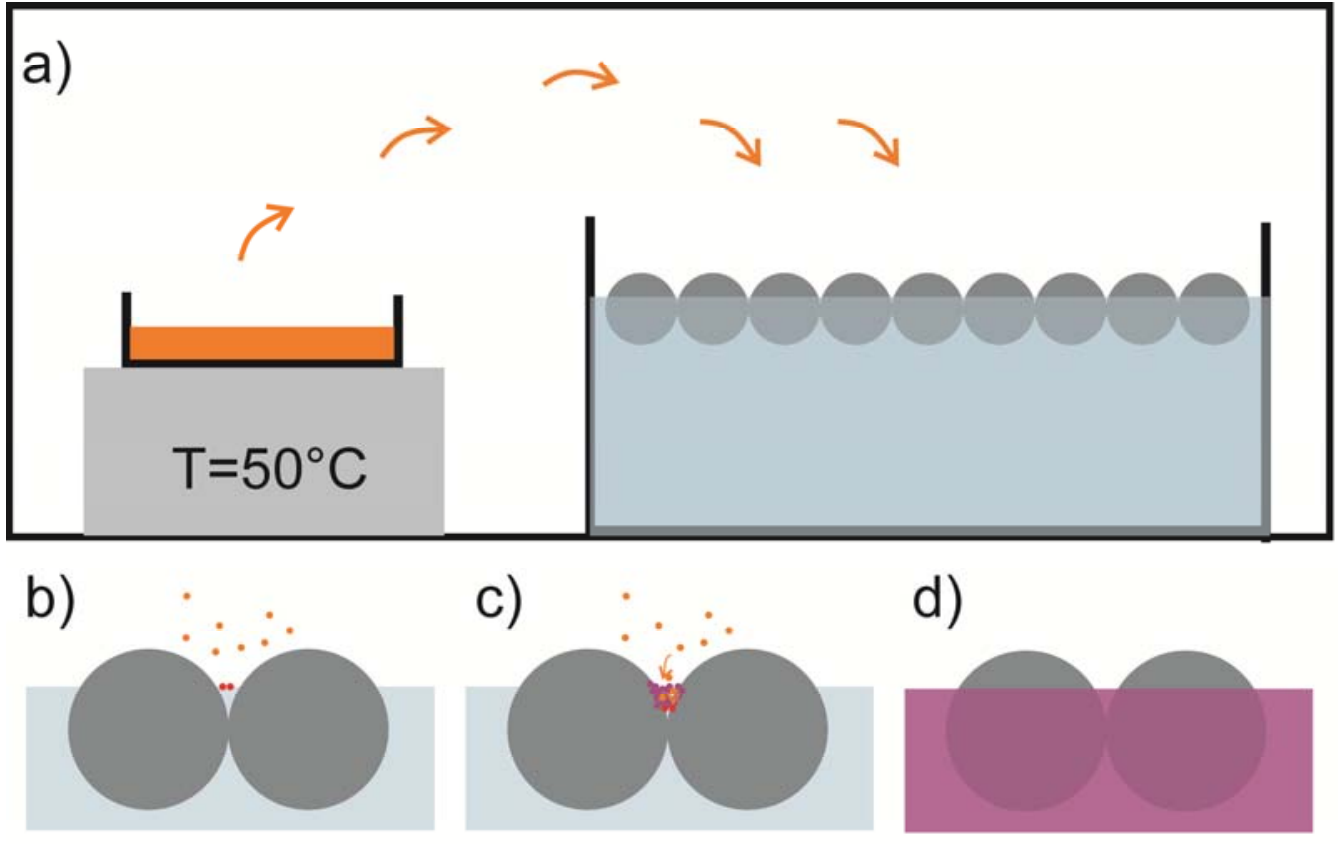

d)

e)<smiles>C=C(C#N)C(=O)OCCCCCCCO</smiles>

n-butyl cyanoacrylate ( $\mathrm{n}-\mathrm{BCA})$<smiles>CCCCOC(=O)C(C#N)CO</smiles><smiles>CCCCOC(=O)C(C)(CC(C)(C)C)C(C)(C)CO</smiles>

Figure 6.5.1 Interfacial polymerization of butyl cyanoacrylate (BCA) used to image the position of colloids at the interface. a) Scheme of the reaction chamber. b-e) schematics of the reaction: BCA molecules (represented as orange dots) diffuse through the vapor phase to the air/water interface. In contact with water, the BCA anion is formed (red dots). The anion initiates the polymerization at the interface to form poly butyl cyanoacrylate (PBCA; purple). As the polymerization proceeds, monomer diffuses through the polymeric phase to the interface where it is polymerized; e) shows the reaction scheme of the polymerization.

\subsection{Experimental details for chapter 5.3}

All processes described here were performed at the University of Ulm by Achim Manzke. Further details can be found in the two publications highlighted in the beginning of chapter 5.3. 


\subsubsection{Non-conventional lithography with platinum containing particles}

\subsubsection{Plasma treatment}

The oxygen plasma was delivered by a commercially available etching machine (Oxford Plasmalab 80 Plus RIE) including an ICP (inductively coupled plasma) source. The following plasma parameters were applied for the pre-treatment of the substrate before depositing the solution on it, as well as for the reduction of the diameter of the particles: $\mathrm{O} 2: 20 \mathrm{sccm}$; ICP power: $100 \mathrm{~W}$; DC-bias: $-12 \mathrm{~V}$; total pressure: $80 \mathrm{mTorr}$; nominal substrate temperature: $20^{\circ} \mathrm{C}$. Before using the isotropic plasma described above, oxygen plasma with a more anisotropic component was used to chop the contact areas between the polymer particles and separate them. This guarantees the conservation of the original hexagonal order during the ashing procedure. The plasma parameters in this case were: O2: $50 \mathrm{sccm}$; ICP power: $200 \mathrm{~W}$; DC bias: $-89 \mathrm{~V}$; total pressure: $80 \mathrm{mTorr}$; nominal substrate temperature: $20^{\circ} \mathrm{C}$. To completely separate the particles, the anisotropic plasma was applied for 20-50 s.

In the RIE-process to etch the silicon substrate, a mixture of CHF3/CF4 $(20: 2 \mathrm{sccm})$ was utilized with a radiofrequency $(\mathrm{RF})$ power of $40 \mathrm{~W}$ and a DC-bias of $-97 \mathrm{~V}$.

\subsubsection{Annealing}

All samples were annealed in a commercially available lamp furnace (UniTemp RTP-1200-100) under the following conditions: oxygen atmosphere; pressure: 2 mbar $(1 \mathrm{mbar}=100 \mathrm{~Pa})$; heating rate: $0.6{ }^{\circ} \mathrm{C} / \mathrm{s}$; maximal temperature: $1100{ }^{\circ} \mathrm{C}$; time at maximal temperature: $10 \mathrm{~min}$.

\subsubsection{Analysis}

The samples were analyzed using the Hitachi S5200 HRSEM with an acceleration voltage of 30 $\mathrm{kV}$ guaranteeing a resolution of $0.5 \mathrm{~nm}$. The images were evaluated further by the program ImageJ. 


\subsubsection{Experimental procedure for iron-platinum containing polystyrene particles}

Monolayer films of precursor-loaded PS particles were exposed to isotropic oxygen plasma $(0.1$ mbar, $100 \mathrm{~W},-11 \mathrm{~V}$ dc-Bias, $300 \mathrm{~K}$ ) delivered by a commercial etching machine (Oxford Plasmalab 80 Plus RIE). The annealing step was undertaken in a commercial rapid thermal annealing furnace (RTP-1200-100, UniTemp). Samples were annealed at $650^{\circ} \mathrm{C}$ for $120 \mathrm{~min}$ in high vacuum and subsequently at $1000{ }^{\circ} \mathrm{C}$ for $10 \mathrm{~min}$ in 1 mbar oxygen atmosphere.

\subsection{Experimental details for chapter 5.3.2 (Plasmonic structures from non-close packed monolayers)}

\subsubsection{Fabrication of arrays of single crescents}

The fabrication process for the crescent arrays was adopted from a similar fabrication process for gold nano-crescents described by Shumaker-Perry ${ }^{[129]}$ and Rochholz. ${ }^{[130]}$ A close packed polystyrene colloid monolayer was deposited on flat glass substrate, and subsequently exposed to oxygen plasma to reduce the size of the particles without affecting the lateral order. For the crescents produced in chapter $\mathrm{xxx}, 550 \mathrm{~nm}$ polystyrene colloids were used and subjected to oxygen plasma (10sccm; $15 \mathrm{~W}$ power) for etching time between 10 and $13 \mathrm{~min}$ in order to create non-close-packed colloidal monolayer masks with diameters as specified in Table 5.3.3. After this step, a thin gold film $(40 \mathrm{~nm})$ was evaporated on the substrate at a tilt angle of $30^{\circ}$ with respect to the surface normal, followed by an ion-etching step normal to the surface. Argon was used as etching gas and the sample was etched until the gold film accessible by the beam was completely vanished. The mask was removed mechanically using Scotch Magic Tape (Scotch Magic Tape, Neuss, France). ${ }^{[130]}$ This process gives rise to arrays of crescent-shaped nanoparticles, uniformly disposed on a substrate in a hexagonal conformation, reflecting the symmetry of the original colloidal mask. 


\subsubsection{Stacked double crescent arrays}

\subsubsection{Colloidal mask fabrication}

Glass slides (Objektträger, Menzel Gläser, Germany, approximately $20 \times 25 \mathrm{~mm}, 1 \mathrm{~mm}$ thick) were cleaned with a 2 vol.-\% detergent solution (Hellmanex, Hellma, Germany) in an ultrasonic bath for $15 \mathrm{~min}$, followed by ultrasonication with utra pure water (milliQ grade) and ethanol. The slides were stored in ethanol and dried in a nitrogen stream prior to use.

\subsection{Spatially separated objects}

As mask, polystyrene colloidal particles with a diameter of $400 \mathrm{~nm}$ were used. The aqueous dispersion of colloids was diluted to $0.01 \mathrm{wt} .-\%$ with ethanol. A drop $(50 \mu \mathrm{l})$ of this dispersion was put on a glass slide and a gentle nitrogen stream was used to dry the sample and thus, randomly distribute the spheres over the substrate, following a procedure developed by Rochholz et al. ${ }^{[130]}$ The surface coverage of particles was approximately 0.15 objects $\cdot \mu \mathrm{m}^{-2}$.

\subsection{Arrays of objects}

Colloids with a diameter of $550 \mathrm{~nm}$ and a carboxylic acid surface functionalization were prepared by surfactant free emulsion polymerization and assembled to a closed packed monolayer at the air water interface following a procedure developed by Retsch et al. ${ }^{[55]}$ Briefly, a 0.5 wt.- $\%$ dispersion of colloids was spincoated onto a parental substrate modified with with $\mathrm{N}$-trimethoxysilylpropyl-N,N,N-trimethylammonium chloride (ABCR/Gelest, Karlsruhe, Germany) to induce positive surface charges. This parental substrate was carefully inserted under an angle of approximately $30^{\circ}$ with respect to the water surface into a water subphase consisting of $450 \mathrm{ml}$ of $\mathrm{H}_{2} \mathrm{O}$ (milliQ grade), $50 \mathrm{ml}$ ammonia (25\% in water) and $70 \mathrm{mg}$ of sodium dodecyl sulfate. The monolayer assembled at the 3 phase contact line and floated off the parental substrate after complete immersion. The monolayer was transferred to the substrate glass slide by manually withdrawing the slide from the water in a way that the monolayer adhered to the glass slide. For electron microscopy investigations, a silicon wafer was used as a substrate. 
The colloidal monolayer was subjected to oxygen plasma ( $10 \mathrm{sccm}$ flow rate, $50 \mathrm{~W}$ power) in a commercially available plasma cleaner (Model femto, Diener electronic, Nagold, Germany) and etched for $13 \mathrm{~min}$ to create a non-close-packed monolayer with size reduced colloids of $400 \mathrm{~nm}$.

\subsubsection{Nanostructure fabrication}

All evaporations were carried out in a Balzers PLS500 evaporation chamber with a Pfeiffer vacuum pump. Subsequently, chromium $(1.5 \mathrm{~nm})$ and gold $(25 \mathrm{~nm})$ were evaporated under an angle of $30^{\circ}$ with respect to the surface normal of the colloid-covered glass slide, followed by evaporation of silicon dioxide $(20 \mathrm{~nm})$, chromium $(1.5 \mathrm{~nm})$ and gold $(25 \mathrm{~nm})$ under an angle of $-30^{\circ}$. Gold and chromium were evaporated at a rate of $0.03 \mathrm{~nm} / \mathrm{s}$, silicon monoxide was evaporated at a rate of $0.1 \mathrm{~nm} / \mathrm{s}$ with an oxygen partial pressure of $1 \cdot 10^{-4} \mathrm{mbar}$ to form silicon dioxide at the sample surface.

The samples were etched with a reactive ion beam (RR-I SQ76, Roth \& Rau, Wüstenbrand, Germany) normal to the surface of the substrate. As etching gas, a mixture of $\mathrm{Ar}, \mathrm{CF}_{4}$ and $\mathrm{O}_{2}$ was used. After the etching procedure, the colloids were removed using elastomeric glue (Marabu Fixogum, Tamm, Germany) that was applied onto the sample surface, hardened for 12 $\mathrm{h}$ at room temperature and then removed mechanically.

\subsubsection{Computer simulations}

All computer simulations were performed by Reza Mohammadi. The optical response of double crescent nanoparticles was calculated using the commercial package JCMwave (Putzbrunn, Germany) that is based on a finite element method (FEM). ${ }^{[256]}$ The software was operated via MATLAB that constructs the geometry, calls the solver and does the post processing. Three dimensional finite element simulations were performed and a perfectly matched layer (PML) method was used to truncate the simulation domain. Tetrahedral adaptive meshes were applied to discretize the computational domain and second order interpolation functions were used on elements. The refractive index of the glass substrate and the silicon dioxide layer separating the crescents was taken as 1.5 . The permittivity of gold was taken from the Drude model: ${ }^{[143]}$

$\varepsilon=\varepsilon_{\infty}-\frac{\omega_{p}^{2}}{\omega^{2}+i \gamma \omega}$

and has been fitted to the data of Johnson and Christy ${ }^{[257]}$ with the following values: 
$\varepsilon_{\infty}=9.63505$

$\omega_{p}=1.365 \cdot 10^{16} \mathrm{rad} / \mathrm{s}$

$\gamma=1.1199 \cdot 10^{14} \mathrm{rad} / \mathrm{s}$

\subsubsection{Opposing double crescent structures}

\subsubsection{Sample preparations}

Glass slides (Objektträger, approximately $20 \times 25 \mathrm{~mm}, 1 \mathrm{~mm}$ thick, Menzel Gläser, Braunschweig, Germany) were cleaned with a 2 vol.-\% detergent solution (Hellmanex II, Hellma GmbH \& Co. KG, Mühlheim, Germany) in an ultrasonic bath for 20 minutes twice. The samples were thoroughly rinsed with ultrapure water and dried in a nitrogen stream. Polystyrene nanospheres, $400 \mathrm{~nm}$ in diameter, were randomly dispersed on the substrate by drop-coating. The solvent was evaporated with a gentle nitrogen stream. An $80 \mathrm{~nm}$ gold layer was evaporated on the glass, the surface normal was perpendicular to the metal source. The samples were etched with an argon ion beam under tilted etching angles between $45^{\circ}$ and $65^{\circ}$ relative to the ion source during the exposure. The etching process was performed in two steps: in the first step, 40 $\mathrm{nm}$ of the gold layer were etched away, then the sample was rotated by $180^{\circ}$, and the remaining $40 \mathrm{~nm}$ of the gold layer were exposed to the ion beam. This yields the two opposing crescent structures. After the etching process, the colloidal mask was removed mechanically with an adhesive tape (Scotch Magic Tape, Neuss, France).

\subsubsection{Computer simulations}

All computer simulations were performed by Reza Mohammadi as described above (chapter 6.7.2.3). 


\subsection{Experimental details for chapter 5.3.3 (Laterally patterned, ultraflat substrates by colloidal lithography and their applications)}

\subsubsection{Fabrication of surface embedded particles}

Embedded nanoparticle arrays have been produced using a modified template-stripping procedure as described by Vogel et al. ${ }^{[188]}$ First, the silicon wafer substrate was passivated by silanization. For that, wafers were cut into pieces of approximately $2 \times 3 \mathrm{~cm}^{2}$ and oxidized by the wet chemical method described in chapter 6.1.3). A monolayer of hexamethyldisilazane (ABCR Speciality Chemicals, Karlsruhe, Germany, used as purchased) was deposited using vapour phase deposition for 30 minutes at ambient pressure and temperature conditions. The colloidal monolayers used as mask were prepared by assembly of the colloids at the air/water interface followed by transfer to the substrate. ${ }^{[55]}$ For gluing, a two component epoxy glue (EPO TEK 377, Epoxy Technologies, Billerica, USA) was used and cured at $150{ }^{\circ} \mathrm{C}$ for $60 \mathrm{~min}$. Before gluing, all glass slides were cleaned by consecutive ultrasonic treatment in $2 \%$ detergent solution (Hellmanex, Mühlheim, Germany), ultra pure water and ethanol. The templatestripping step was performed by carefully scratching on the edges of the silicon wafer glued to the glass slide with a scalpel until the wafer could be lifted off the substrate.

\subsubsection{Layer by layer deposition of polyelectrolytes}

The initial cysteamine monolayer, used to create positive charges on the substrate, was assembled by immersion of the substrate in a $2 \mathrm{mg} \cdot \mathrm{ml}^{-1}$ solution of cysteamine hydrochloride (Sigma Aldrich, Seelze, Germany) in ultrapure water. Subsequent layer by layer deposition was performed by immersion for 20 minutes in solutions of positively- and negatively charged polyelectrolytes.

Positively charged polyelectrolyte solutions: $50 \mathrm{ml}$ ultra pure water, $0.094 \mathrm{~g}$ polyallylamine hydrochloride ("PA", MW = 15,000, $10.29 \mathrm{~g} \mathrm{NaBr}, 0.5 \mathrm{ml} \mathrm{HCl}\left(0.1 \mathrm{~mol} \cdot 1^{-1}\right)$. Negatively charged polyelectrolyte: $50 \mathrm{ml}$ ultra pure water, $0.207 \mathrm{~g}$ sodium polystyrene sulfonate, ("PSS", $\mathrm{MW}=70,000), 4.049 \mathrm{~g} \mathrm{MnCl}_{2}, 0.5 \mathrm{ml} \mathrm{HCl}\left(0.1 \mathrm{~mol} \cdot \mathrm{l}^{-1}\right)$. All chemicals were obtained from Sigma Aldrich, Seelze, Germany. 


\subsubsection{Nanoscale patterning of bilayer model membranes}

\subsubsection{Substrate preparation}

The substrates were constructed by a similar preparation process as described in chapter 6.8.1 with minor modifications. As the substrates were used for fluorescence recovery after photobleaching experiments, the autofluorescence of the epoxy glue had to be blocked in the experiments. Hence, a second layer of $1.5 \mathrm{~nm}$ chromium and100 nm gold was evaporated on top of the silicon dioxide layer. Afterwards, the wafer template with the layered structures was glued to a glass slide in a similar was as described before and mechanical rupture was induced by a scalpel in order to reveal the patterned, ultraflat surface. For different experiments, either sparsely distributed silica beads ( $2 \mu \mathrm{m}$ in diameter, Duke Scientific Corp., CA, USA) or densely packed colloidal monolayers were used as masks

\subsubsection{Vesicle fusion}

1,2-dioleoyl-sn-glycero-3-phosphoethanolamine-N-(7-nitro-2-1,3-benzoxadiazol-4-yl) (DOPENBD) and 1,2-diphytanoyl-sn-glycero-3-phosphocholine (DPhyPC) were purchased from Avanti Polar Lipids (Alabaster, AL, USA) and used as received. Small unilamellar vesicles (50 $\mathrm{nm}$ in diameter) were prepared by extrusion of a $2 \mathrm{mg} / \mathrm{ml}$ lipid solution (DPhyPC and $0.8 \mathrm{~mol} \%$ DOPE-NBD) in ultra-pure water ( $>18 \mathrm{M} \Omega \mathrm{cm}$, Millipore, Schwalbach, Germany). The vesicles were stored at $4{ }^{\circ} \mathrm{C}$ and used within 2 days. Vesicle fusion was performed in $0.1 \mathrm{M}$ $\mathrm{KCl}$ solution.

\subsubsection{Lipid self-assembly}

Self-assembly on the the patterned substrates was achieved by a simple self-assembly process to form a monolayer selectively on the gold part of the substrates. The polymerizable thiolipids were self-assembled by immersion of the sample in a $0.2 \mathrm{mg} / \mathrm{ml}$ ethanolic solution of the lipid for 24 hours, followed by thorough rinsing with ethanol. Polymerization was achieved by UV illumination at $254 \mathrm{~nm}$ at room temperature under $\mathrm{N}_{2}$ atmosphere, using a $6 \mathrm{~W}$ laboratory hand lamp at a distance of $5 \mathrm{~cm}$, delivering $5.8 \mathrm{~mW} \cdot \mathrm{cm}^{-2}$. 


\subsubsection{Fluorescence Recovery after Photobleaching}

FRAP experiments were performed by Dr. Mathieu Jung using a customized set-up based on an Olympus IX70 inverted microscope equipped with a photomultiplier (Type B2F/RFI, Electron Tubes Ltd., Middex, UK). The samples were illuminated with a mercury lamp integrated in the microscope for the observation and focusing, whereas the FRAP measurements were performed with the $488 \mathrm{~nm}$ line of an argon ion laser, creating a circular spot (Ø $4.2 \mu \mathrm{m})$. All measurements were conducted at room temperature in PBS buffer, and repeated at least 15 times for each membrane.

Diffusion coefficients were determined by adjusting the experimental data to a model developed by Soumpasis: ${ }^{[297]}$

$$
f_{k}(t)=F_{k}(\infty)-\left[F_{k}(\infty)-F_{k}(0)\right] \cdot\left\{1-\exp \left(\frac{-\omega^{2}}{2 D t}\right) \cdot\left[I_{0}\left(\frac{\omega^{2}}{2 D t}\right)+I_{1}\left(\frac{\omega^{2}}{2 D t}\right)\right]\right\}
$$

where $\omega$ is the radius of the bleaching spot, $t$ is the time, $F_{k}(0)$ is the minimum fluorescence intensity after photobleaching, $f_{k}(t)$ is the fluorescence intensity at time $t$ and $F_{k}(\infty)$ is the fluorescence intensity as $t$ reaches $\infty$ and $I_{0}, I_{t}$ are spherical Bessel functions of zero and first order, and $D$ the diffusion coefficient.

The relative recovery, indicating the fraction of mobile lipids within the measured leaflet is defined as:

$$
\text { Relative Recovery }=\frac{F_{\infty}-F_{0}}{F_{-}-F_{0}} \cdot 100 \%
$$

where $F_{\text {- }}$ is the fluorescence intensity before bleaching, $F_{0}$ is the fluorescence immediately after bleaching and $F_{\infty}$ is the recovered fluorescence intensity. 


\section{Summary and Outlook}

The motivation and unifying basis for the different projects presented in this thesis was to develop approaches and techniques to create surface patterns based on the assembly of colloidal particles in two dimensions. Two distinct approaches were pursuit. First, the focus was set on the self-assembly processes of colloids. Crystallization approaches were developed to simplify the fabrication of classical, single-size particle monolayers and to produce more complex architectures in a controlled way. Second, innovative approaches to lithography were elaborated with the aim to introduce specific functions or structural features to the nanostructure arrays that set them apart from existing approaches and add to their utility.

In the following, a short summary of the reached achievements is given followed by a short outlook of possible research directions based on this thesis.

\section{Monolayer assembly processes}

\section{“Monolayers to go"}

Though there are numerous monolayer crystallization processes available, the reliable generation of crystals with a high long range order over large areas is still not trivial. One of the most powerful techniques to create crystals with the aforementioned properties is the assembly on a Langmuir trough. Yet, it requires special equipment not readily available in every lab and is rather time-consuming. In this thesis, a method to directly assemble colloidal particles into close-packed monolayers with high long range order was developed. In analogy to the assembly on a Langmuir trough, the air/water interface is used to template the assembly process and the monolayer can be transferred manually to arbitrary substrates. The direct assembly is driven by the balance between attractive capillary forces needed to drive the particles together and sufficient electrostatic and dipole repulsion that is required to provide time for the particles to find their minimum free energy position. It has been recognized that this balance can be positively affected by the presence of small amounts of surfactants on the surface or adjustment of the $\mathrm{pH}$ value of the subphase.

It is believed that this method represents a valuable contribution to a relatively new research area I coined "monolayers to go". This includes crystallization methods that are reliable, fast and experimentally easy and were developed with the aim of making colloidal monolayers available to a wide range of researchers. The developed method does not require any special equipment and can be performed wherever needed - from labs around the globe to classrooms or labcourses. 


\section{Non-close-packed architectures}

The close-packed monolayers were subjected to a plasma-induced size reduction to yield nonclose-packed structures that are valuable for colloidal lithography as will be shown below. A commercial plasma cleaner, available in almost every surface-science related laboratory, was used for the etching experiments. All important parameters of the etching process were investigated in order to provide a methodology that is amenable to a broad range of researchers.

A strong change in the monolayer color was observed upon size reduction of the individual colloids. UV/Vis spectroscopy was used to characterize the optical properties of such non-closepacked architecture on glass and a simple model based on geometric considerations was derived to explain the spectral features. Using this method, the size and inter-colloid spacing can be estimated from the optical properties without the use of electron microscopy.

\section{Binary monolayers}

The air/water interface on a Langmuir trough was used to co-assembly binary monolayers comprised of a close-packed monolayer of large and small spheres in the interstitial sites. Of key importance for this project was the quantification of the interface fraction of the colloids from the surface-pressure area isotherms. Knowledge of this interface fraction of the individual colloids enables the precise adjustment of the stoichiometry of large and small spheres at the interface. Using this novel method, binary structures with $\mathrm{LS}_{2}, \mathrm{LS}_{6}$ and $\mathrm{LS}_{9}$ configurations were prepared by co-assembly of colloids with a large range of size ratios between 0.19 and 0.41 . The dimensions of the binary monolayer were limited only by the trough dimensions. Deposition on arbitrary substrates and surface topographies was demonstrated as well. Furthermore, in the course of the project, a method was developed to visualize the contact angle of colloids at the interface by embedding in a polymer matrix.

\section{Lithographic processes}

\section{Non-conventional lithography}

The non-conventional lithography process differs from classical lithography as functional, metal-complex containing colloidal particles are applied. These are not used as masks but rather as sacrificial materials that are combusted to give rise to metal nanoparticle arrays. In this thesis, two key features of this process were experimentally realized. First, it was shown that both size and inter-particle distance in the array are solely determined by the properties of the colloids and can be tuned accordingly. In contrast to classical colloidal lithography, very small metal 
particle sizes with no inherent lower limit can be produced. Using colloids with varying platinum content, the final dot size was precisely adjusted between 8 and $15 \mathrm{~nm}$. The interparticle distance was adjusted between $132 \mathrm{~nm}$ and $271 \mathrm{~nm}$ by using size-increased emulsion particles prepared by a seeded emulsion polymerization process.

Second, it was shown that the process can also be used to generate more complex inorganic particles not available with the classical colloidal lithography process. This was demonstrated exemplarily for magnetic FePt alloy nanoparticles from precursor latex particles containing stoichiometric amounts of $\mathrm{Fe}(\mathrm{acac})_{3}$ and $\mathrm{Pt}(\mathrm{acac})_{2}$.

The syntheses of the metal-containing precursor latex particles led to various interesting discoveries. It was shown that the reliable determination of the metal contents of the colloids by inductively coupled plasma optical emission spectrometry is non-trivial and requires the addition of high amounts of surfactants. Additionally, it was discovered that the symmetric metal-acetylacetonate are interfacially active to different extends. A model to explain the observation based on hydrogen-bonding of water to the complex, partially followed by condensation reactions was proposed and experimentally verified.

\section{Plasmon dimer architectures}

The non-close-packed monolayers described above were used in a lithographic process to construct arrays of nano-crescent dimer units with defined mutual orientation. The placement of nanoscale metallic objects in close proximity with a defined orientation gives rise to a coupling process of the plasmon resonances that induces strong shifts of the resonance wavelengths and causes strong near-field enhancements in small volumes between the two crescents.

The key innovation of the process is the use of a single colloid to create two crescent-shaped objects. The colloid mask thus defines the mutual orientation and enables the precise placement of the two objects in close proximity.

Two different architectures were developed. First, a vertical alignment of two crescents with their tips being placed on top of each other was demonstrated. The process thus provides a colloidal lithographic approach to yield a quasi-3D architecture of crescents formerly only available with electron beam lithography. Second, the crescent dimers were laterally oriented in the substrate plane with their tips facing each other. The sharp curvature paired with the small gap in between the tips gives rise to a strongly enhanced near-field in the small volume of the gap.

A plasmon hybridization model to explain the shifts of the plasmon resonances upon coupling was elaborated for all polarization dependent resonances. 


\section{Ultraflat substrates}

Colloidal lithography was applied as well to produce surface-embedded nanoparticle arrays by a template-stripping method. In this approach, the specific property of the arrays is the absence of any surface topography: gold nanotriangles are homogeneously embedded in a silicon dioxide matrix with smooth transitions between the materials and a root-mean-square surface roughness of only several angstroms. The properties of this architecture were exploited in two applications. The strong adhesion of the nanostructures to the substrate caused by the embedding into the matrix was used to create a very robust sensing architecture. Deposition and subsequent mechanical removal of polyelectrolyte films was performed and monitored by the shifts of the plasmon resonances. Thus, re-usability of a localized surface plasmon resonance sensor was demonstrated.

In a different application, the low surface roughness was exploited to produce patterned model membrane architectures. Photopolymerizable anchor lipids were assembled onto the gold nanoparticles and the bilayer membrane was completed by vesicle fusion. Thus, an architecture comprising of a fluid lipid bilayer membrane with nanoscale diffusion barriers homogeneously merged into the proximal leaflet was produced.

\section{Outlook}

The projects introduced in this thesis combine sophisticated structural properties with the simplicity of fabrication by colloidal lithography. As thus, they might be exploited in several applications only touched in this thesis. Yet, plenty of work can be dedicated to further refine or extent the different methods in order to add more sophisticated functions. As such, functional colloidal particles with attractive materials properties could be used for the co-assembly of binary monolayers in order to combine advanced materials properties with defined spatial arrangements provided by the configuration of the binary monolayer. Additionally, such bilayers could also be stacked into three-dimensional architectures with possible applications in photonics or phononics. Another feature of the process; the possibility to structure topographic substrates has not yet been further exploited but would provide an efficient route towards hierarchical materials.

The non-conventional lithography approach is potentially attractive as a wide range of materials can be patterned with nanometer precision. As demonstrated for iron-platinum alloys, also complex materials can be generated. This approach could be extended into a variety of material classes as for example complex metal-oxides or quantum dots. Ongoing investigations exploit 
the possibility of creating arrays of materials with a stronger tendency towards oxidation. Finally, research efforts to create gold nanoparticles have been initiated. Up to this point, the low stability of applied gold complexes has impeded a successful incorporation. As an alternative approach, the incorporation of gold nanoparticles with subsequent combustion of the organic shell is pursuit.

The plasmonic properties of the crescent dimers merit to be exploited in applications were strong near-field enhancements are desired - most prominently in fluorescence enhancement and surface enhanced Raman spectroscopy. Furthermore, steps towards an experimental observation of the dark modes merit further research efforts. A possible way for their observation is to induce asymmetries to the structures that add dipole moments to the dark hybrid mode and thus, strengthen the interaction with the electric field of the incident light. 


\section{List of Abbreviations}

$\sigma$

$\gamma$

$\varepsilon$

AA

AFM

APS

d

DCP

e.g.

Ext.

FRAP

i.e.

KPS

$\mathrm{LS}_{\mathrm{n}}$

LSPR

n

NapSS

ncp

NSL

PMMA

PS

r

RIE

RMS

SDS

SDT

SAM

$\mathrm{sccm}$

SEM

TSG

UV/Vis-NIR

V59

wt.- $\%$ surface tension

interface tension

dielectric function / permittivity

acrylic acid

atomic force microscopy

ammomium peroxodisulfate

diameter

dichlorophenole

exempli gratia; for example

Extinction

fluorescence recovery after photobleaching

id est; that is

potassium peroxodisulfate

large sphere-small sphere ratio

localized surface plasmon resonance

refractive index

sodium para-styrene sulfonate

non-close-packed

nanosphere lithography

poly(methyl-methacrylate)

polystyrene

radius

reactive ion etching

root mean square

sodium dodecyl sulfate

spinning drop tensionmetry

self-assembled monolayer

standard cubic centimeters per minute

Scanning electron microscopy

template stripped gold

ultraviolet-visible-near infrared

2,2'-azobis(2-methylbutyronitrile)

weight-percent 


\section{Acknowledgements}

Many people contributed to this thesis in one way or another and I am expressing my gratitude to all of them. I enjoyed working at the Max Planck Institute and, during a long stay, I met many wonderful colleagues that eventually became close friends. I will treasure the atmosphere in the institute, especially in my office and the coffee corners, the weekly football matches and uncountable barbecue parties.

First of all, I want to thank Katharina Landfester for offering me the possibility to work in her group and explore the wonderful world of colloidal monolayers. I am very grateful for guidance, discussions, ideas and support of my supervisor Clemens Weiss.

I am indebted to the Materials Science in Mainz graduate school that funded me during the complete time as a $\mathrm{PhD}$ student and sponsored my participation in several conferences and summer schools.

Uli Jonas and Max Kreiter always had patience and an open ear for my questions and I benefitted enormously from fruitful discussions, long explanations and their enthusiasm for science.

Many additional cooperation partners were involved in the various projects presented in this thesis and I highly appreciated all the contributions from Achim Manzke, Ulrich Ziener, Alfred Plettl, Janina Fischer, Markus Retsch, Mathieu Jung, Ingo Köper, Noelia Bocchio, Coenrad van den Brom and Christoph Hauser and Laurence de Viguerie.

I want to especially underline the help of Reza Mohammadi and Andreas Unger who performed all computer simulations presented in this thesis; a task that I could have never accomplished myself.

Sebastian Goerres did a great job as HiWi and really helped me with spinning drop measurements and monolayer etching experiments.

The technical support at the institute was always perfect. Amongst the many people maintaining equipment and infrastructure, I want to especially thank Gunnar Glaser, Uwe Rietzler, HansJosef Beauvisage, Elke Muth and Gabrielle Schäfer.

I spent wonderful weeks in Crete in spring 2010 and I want to express my gratitude to Dimitris Vlassopoulos and Uli Jonas for making this stay possible and being such great hosts. The whole 
group of Dimitris made me feel very welcome and I especially want to thank Laurence and Kiki for long hours in the lab, at the beaches, in the mountains and in various tavernas.

I also want to thank Clemens, Steffi, Daniel, Schmelzi, Nini, Uli and Adrian for meticulously proof-reading this thesis and my mates Anke, Nermeen, Vero, Daniel, Max and Christoph for all the help, support and fun in the best office of the institute.

Finally, what would life be without friends? Thank you for everything!

Amongst them all, I only want to mention my family, especially my parents, as well as Micha, Daniel and Steffi who are more important to me than words could possibly describe. 


\section{Curriculum Vitae and List of Publications}

\subsection{Curriculum Vitae}

Name:

Nicolas Vogel

Date of birth:

20.10.1981

Place of birth:

Mainz

Education:

$09 / 1993-03 / 2001$

Gymnasium Nieder-Olm (high school)

Abitur (A-levels)

$04 / 2002-01 / 2008$

Johannes Gutenberg University Mainz, Mainz, Germany

Academic studies

Degree: diploma-chemist

08/2005 - 03/2006 Seoul National University, Seoul, Republic of Korea

Visiting student in the group of Prof. Dr. Do-Young Yoon

Topic: High k Dielectric Materials as Gate Insulators in Organic Thin Film Transistors

05/2007 - 01/2008 Max-Planck-Institute for Polymer Research, Mainz, Germany

Diploma thesis in the group of Prof. Wolfgang Knoll

Topic: Heterogeneously Patterned, Ultraflat Surfaces: Construction and Applications

08/2008 - 07/2011 Max-Planck-Institute for Polymer Research, Mainz, Germany

$\mathrm{PhD}$ student in the group of Prof. Katharina Landfester

04/2010 - 06/2010 Foundation for Research and Technology Hellas (FORTH), Iraklio, Crete, Greece

Visiting scientist in the group of Prof. Dimitris Vlassopoulos

Topic: Langmuir-Blodgett Films of Colloidal Monolayers and Interfacial Stress Rheology 


\subsection{Scientific publications related to results presented in this thesis}

N. Vogel, M. Jung, N.L. Bocchio, M. Retsch, M. Kreiter and I. Köper,

Reusable Localized Surface Plasmon Sensors based on Ultrastable Nanostructures

SMALL 2010, 6, 104

N. Vogel, C. P. Hauser, K. Schuller, K. Landfester and C.K. Weiss, Accurate Elemental Analysis of Metal-containing Polymer Latexes using ICP Optical Emission Spectroscopy Macromolecular Chemistry and Physics 2010, 12, 1355 (cover story of the issue)

N. Vogel*, J. Fischer*, R. Mohammadi, M. Retsch, H.-J. Butt, K.Landfester, C..K. Weiss and Max Kreiter (*equal contribution)

Plasmon Hybridization in Stacked Double Crescents Arrays Fabricated by Colloidal Lithography

Nano Letters 2011, 11, 446

M. Jung, N. Vogel and I. Köper, Template Assisted Patterning of Solid Supported Membranes Langmuir 2011, 27, 7008

A. Manzke*, N. Vogel*, C.K. Weiss, U. Ziener, A. Plettl, K. Landfester and

P. Ziemann (*equal contribution)

Precise Control of Size and Separation Distance of Platinum Nanoparticles Fabricated by Emulsion Polymerization Based Colloidal Lithography

Nanoscale 2011, 3, 2523

N. Vogel, U. Jonas, K. Landfester and C.K. Weiss

Wafer Scale Fabrication of Binary Colloidal Crystals with Controlled Stoichiometric Compositions

Advanced Functional Materials, accepted for publication 
N. Vogel, S. Goerres, K. Landfester and C.K. Weiss

A Convenient Method to Produce Close- and Non-close-packed Colloidal Monolayers Using Direct Assembly at the Air/water Interface and Subsequent Plasma Induced Size Reduction Macromolecular Chemistry and Physics, accepted for publication

C.R. Van den Brom, N. Vogel, C.P. Hauser, S. Goerres, M. Wagner, K. Landfester and C.K. Weiss

Unexpected Interfacial Activity in Highly Symmetric Metal $\beta$-diketonate complexes

Langmuir, accepted for publication

N. Vogel, U. Ziener, A. Manzke, A. Plettl, P. Ziemann, J. Biskupek, C.K. Weiss and K. Landfester

Platinum Nanoparticles from Size Adjusted Functional Colloidal Particles Generated by a Seeded Emulsion Polymerization Process

submitted

A. Manzke, A. Plettl, U. Wiedwald, L. Han, P. Ziemann, E. Schreiber, U. Ziener, N. Vogel, C.K. Weiss, K. Landfester, K. Fauth, J. Biskupek and U. Kaiser

Formation of Highly Ordered Alloy Nanoparticles Based on Precursor-filled Latex Spheres

submitted

11 J. Fischer*, N. Vogel*, R. Mohammadi, C.K. Weiss, K. Landfester and M. Kreiter (*equal contribution)

Construction and Optical Properties of Opposing Nanocrescent Dimer Structures in Close Proximity

submitted 


\subsection{Conference Contributions}

02/2009 Trends in Nanoscience, Kloster Irsee, Germany

Poster presentation:

Fabrication of Monodisperse Metal-Containing Colloidal Particles for Applications in Non-conventional Lithography

03/2009 Hybrid Materials Conference, Tours, France

Oral presentation:

Laterally Patterned, Ultraflat Surfaces: Construction and Applications

06/2009 Frontiers in Polymer Science, Mainz, Germany

Poster presentation:

The Miniemulsion Technique as Versatile Instrument for the Preparation of

Nanocomposites

07/2010 Soft Matter Conference, Granada, Spain

Oral presentation:

Soft Matter Going Hard: Unconventional Lithography with Miniemulsion Particles

06/2011 International Workshop on Nanoplasmonics, Vigo, Spain

Poster presentation:

Coupling of Plasmon Modes in Nano-Crescent Dimer Structures Fabricated by Colloidal Lithography

07/2011 UK Colloids Conference, London, United Kingdom

Oral presentation:

Crystallization of binary colloidal monolayers with adjustable configurations at the air/water interface 


\section{Bibliography}

[1] R. P. Feynman, presented at the "Annual Meeting of the American Physical Society", Pasadena, CA, USA 1959.

[2] G. Whitesides, F. Frankel, No Small Matter - Science on the Nanoscale, Harvard University Press, Cambridge, MA, USA, 2009.

[3] P. Pieranski, Phys. Rev. Lett. 1980, 45, 569-572.

[4] U. C. Fischer, H. P. Zingsheim, Journal of Vacuum Science \& Technology 1981, 19, 881-885.

[5] J. C. Hulteen, R. P. Vanduyne, J. Vac. Sci. Technol. A-Vac. Surf. Films 1995, 13, $1553-$ 1558.

[6] J. C. Hulteen, D. A. Treichel, M. T. Smith, M. L. Duval, T. R. Jensen, R. P. Van Duyne, J. Phys. Chem. B 1999, 103, 3854-3863.

[7] T. R. Jensen, G. C. Schatz, R. P. Van Duyne, J. Phys. Chem. B 1999, 103, 2394-2401.

[8] T. R. Jensen, M. D. Malinsky, C. L. Haynes, R. P. Van Duyne, J. Phys. Chem. B 2000, 104, 10549-10556.

[9] Y. Li, W. Cai, G. Duan, Chem. Mat. 2008, 20, 615-624.

[10] J. H. Zhang, Y. F. Li, X. M. Zhang, B. Yang, Adv. Mater. 2010, 22, 4249-4269.

[11] J. Fischer, N. Bocchio, A. Unger, H. J. Butt, K. Koynov, M. Kreiter, J. Phys. Chem. C 2010, 114, 20968-20973.

[12] J. P. Camden, J. A. Dieringer, J. Zhao, R. P. Van Duyne, Accounts Chem. Res. 2008, 41, 1653-1661.

[13] M. Kolle, P. M. Salgard-Cunha, M. R. J. Scherer, F. M. Huang, P. Vukusic, S. Mahajan, J. J. Baumberg, U. Steiner, Nat. Nanotechnol. 2010, 5, 511-515.

[14] S. G. Park, S. Y. Lee, S. G. Jang, S. M. Yang, Langmuir 2010, 26, 5295-5299.

[15] W. L. Min, B. Jiang, P. Jiang, Adv. Mater. 2008, 20, 3914.

[16] C. Selhuber-Unkel, T. Erdmann, M. Lopez-Garcia, H. Kessler, U. S. Schwarz, J. P. Spatz, Biophys. J. 2010, 98, 543-551.

[17] P. S. Weiss, ACS Nano 2007, 1, 73-78.

[18] K. Landfester, Macromol. Rapid Commun. 2001, 22, 896-936.

[19] K. Landfester, Ann. Rev. Mater. Res. 2006, 36, 231-279.

[20] K. Landfester, Angew. Chem.-Int. Edit. 2009, 48, 4488-4507.

[21] K. Landfester, N. Bechthold, S. Forster, M. Antonietti, Macromol. Rapid Commun. 1999, 20, 81-84.

[22] K. Landfester, N. Bechthold, F. Tiarks, M. Antonietti, Macromolecules 1999, 32, 5222 5228 .

[23] C. K. Weiss, K. Landfester, in Hybrid Latex Particles: Preparation With, Vol. 233, Springer-Verlag Berlin, Berlin, 2010, pp. 185-236.

[24] M. R. Lorenz, M. V. Kohnle, M. Dass, P. Walther, A. Hocherl, U. Ziener, K. Landfester, V. Mailander, Macromol. Biosci. 2008, 8, 711-727.

[25] S. Dhar, F. X. Gu, R. Langer, O. C. Farokhzad, S. J. Lippard, Proc. Natl. Acad. Sci. U. S. A. 2008, 105, 17356-17361.

[26] E. Schreiber, U. Ziener, A. Manzke, A. Plettl, P. Ziemann, K. Landfester, Chem. Mat. 2009, 21, 1750-1760.

[27] M. Urban, A. Musyanovych, K. Landfester, Macromol. Chem. Phys. 2009, 210, 961970.

[28] W. D. Harkins, J. Am. Chem. Soc. 1947, 69, 1428.

[29] J. W. Goodwin, J. Hearn, C. C. Ho, R. H. Ottewill, Colloid Polym. Sci. 1974, 252, 464471.

[30] F. K. Hansen, J. Ugelstad, J. Polym. Sci. Pol. Chem. 1978, 16, 1953-1979.

[31] F. K. Hansen, J. Ugelstad, J. Polym. Sci. Pol. Chem. 1979, 17, 3033-3045.

[32] B. Derjaguin, L. Landau, Acta Physicochim. URSS 1941, 14, 633. 
[33] E. W. Verwey, J. T. G. Overbeek, Theory of the stability of lyophobic colloids, Elsevier, Amsterdam, 1948.

[34] H. J. Butt, K. Graf, M. Kappl, Physics and Chemistry of Interfaces, Wiley-VCH, Weinheim, 2006.

[35] T. F. Tadros, Colloid Stability: The role of Surface Forces I, Wiley-VCH, Weinheim, 2007.

[36] E. M. Lifshitz, Soviet Physics Jetp-Ussr 1956, 2, 73-83.

[37] O. Stern, Z. Elektrochem. Angew. Phys. Chem. 1924, 30, 508-516.

[38] Q. Li, U. Jonas, X. S. Zhao, M. Kapp, Asia-Pac. J. Chem. Eng. 2008, 3, 255-268.

[39] E. Sheppard, N. Tcheurekdjian, Journal of Colloid and Interface Science, 28, 481-486.

[40] D. Y. C. Chan, J. D. Henry, L. R. White, Journal of Colloid and Interface Science 1981, 79, 410-418.

[41] P. A. Kralchevsky, K. Nagayama, Langmuir 1994, 10, 23-36.

[42] P. A. Kralchevsky, K. Nagayama, Adv. Colloid Interface Sci. 2000, 85, 145-192.

[43] O. D. Velev, S. Gupta, Adv. Mater. 2009, 21, 1897-1905.

[44] F. Li, D. P. Josephson, A. Stein, Angew. Chem.-Int. Edit. 2011, 50, 360-388.

[45] A. J. Haes, R. P. Van Duyne, J. Am. Chem. Soc. 2002, 124, 10596-10604.

[46] A. J. Haes, S. L. Zou, G. C. Schatz, R. P. Van Duyne, J. Phys. Chem. B 2004, 108, 109116.

[47] A. J. Haes, S. L. Zou, G. C. Schatz, R. P. Van Duyne, J. Phys. Chem. B 2004, 108, 6961-6968.

[48] N. Denkov, O. Velev, P. Kralchevski, I. Ivanov, H. Yoshimura, K. Nagayama, Langmuir 1992, 8, 3183-3190.

[49] N. D. Denkov, O. D. Velev, P. A. Kralchevsky, I. B. Ivanov, H. Yoshimura, K. Nagayama, Nature 1993, 361, 26.

[50] A. S. Dimitrov, C. D. Dushkin, H. Yoshimura, K. Nagayama, Langmuir 1994, 10, 432440.

[51] R. Micheletto, H. Fukuda, M. Ohtsu, Langmuir 1995, 11, 3333-3336.

[52] A. S. Dimitrov, K. Nagayama, Langmuir 1996, 12, 1303-1311.

[53] L. M. Goldenberg, J. Wagner, J. Stumpe, B. R. Paulke, E. Gornitz, Langmuir 2002, 18, 3319-3323.

[54] J. J. Wang, Q. Li, W. Knoll, U. Jonas, J. Am. Chem. Soc. 2006, 128, 15606-15607.

[55] M. Retsch, Z. C. Zhou, S. Rivera, M. Kappl, X. S. Zhao, U. Jonas, Q. Li, Macromol. Chem. Phys. 2009, 210, 230-241.

[56] L. Malaquin, T. Kraus, H. Schmid, E. Delamarche, H. Wolf, Langmuir 2007, 23, 11513-11521.

[57] B. G. Prevo, O. D. Velev, Langmuir 2004, 20, 2099-2107.

[58] P. Jiang, M. J. McFarland, J. Am. Chem. Soc. 2004, 126, 13778-13786.

[59] P. Jiang, T. Prasad, M. J. McFarland, V. L. Colvin, Appl. Phys. Lett. 2006, 89.

[60] A. Mihi, M. Ocaña, H. Míguez, Adv. Mater. 2006, 18, 2244-2249.

[61] K. M. Chen, X. Jiang, L. C. Kimerling, P. T. Hammond, Langmuir 2000, 16, 78257834.

[62] U. Jonas, A. del Campo, C. Kruger, G. Glasser, D. Boos, Proc. Natl. Acad. Sci. U. S. A. 2002, 99, 5034-5039.

[63] X. Zhang, J. Zhang, D. Zhu, X. Li, X. Zhang, T. Wang, B. Yang, Langmuir 2010, 26, 17936-17942.

[64] E. Kumacheva, R. K. Golding, M. Allard, E. H. Sargent, Adv. Mater. 2002, 14, 221.

[65] N. V. Dziomkina, G. J. Vancso, Soft Matter 2005, 1, 265-279.

[66] K. Q. Zhang, X. Y. Liu, Nature 2004, 429, 739-743.

[67] S. O. Lumsdon, E. W. Kaler, J. P. Williams, O. D. Velev, Appl. Phys. Lett. 2003, 82, 949-951.

[68] R. Mayoral, J. Requena, J. S. Moya, C. Lopez, A. Cintas, H. Miguez, F. Meseguer, L. Vazquez, M. Holgado, A. Blanco, Adv. Mater. 1997, 9, 257.

[69] H. Miguez, F. Meseguer, C. Lopez, A. Mifsud, J. S. Moya, L. Vazquez, Langmuir 1997, 13, 6009-6011. 
[70] S. H. Park, D. Qin, Y. Xia, Adv. Mater. 1998, 10, 1028.

[71] R. Aveyard, J. H. Clint, D. Nees, V. N. Paunov, Langmuir 2000, 16, 1969-1979.

[72] S. M. Weekes, F. Y. Ogrin, W. A. Murray, P. S. Keatley, Langmuir 2007, 23, 10571060 .

[73] N. Vogel, L. de Viguerie, U. Jonas, C. Weiss, K. Landfester, Adv. Funct. Mater. 2011, accepted for publication.

[74] L. Isa, K. Kumar, M. Muller, J. Grolig, M. Textor, E. Reimhult, ACS Nano 2010, 4, 5665-5670.

[75] R. McGorty, J. Fung, D. Kaz, V. N. Manoharan, Mater. Today 2010, 13, 34-42.

[76] E. Sheppard, Tcheurek.N, Kolloid-Zeitschrift and Zeitschrift Fur Polymere 1968, 225, 162.

[77] K. U. Fulda, B. Tieke, Adv. Mater. 1994, 6, 288-290.

[78] K. U. Fulda, B. Tieke, Supramol. Sci. 1997, 4, 265-273.

[79] M. Szekeres, O. Kamalin, P. G. Grobet, R. A. Schoonheydt, K. Wostyn, K. Clays, A. Persoons, I. Dekany, Colloid Surf. A-Physicochem. Eng. Asp. 2003, 227, 77-83.

[80] M. Bardosova, M. E. Pemble, I. M. Povey, R. H. Tredgold, Adv. Mater. 2010, 22, 31043124.

[81] F. Lenzmann, K. Li, A. H. Kitai, H. D. H. Stover, Chem. Mat. 1994, 6, 156-159.

[82] B. van Duffel, R. H. A. Ras, F. C. De Schryver, R. A. Schoonheydt, Journal of Materials Chemistry 2001, 11, 3333-3336.

[83] K. Muramatsu, M. Takahashi, K. Tajima, K. Kobayashi, Journal of Colloid and Interface Science 2001, 242, 127-132.

[84] P. Masse, S. Ravaine, Colloid Surf. A-Physicochem. Eng. Asp. 2005, 270, 148-152.

[85] N. Nagy, A. E. Pap, E. Horvath, J. Volk, I. Barsony, A. Deak, Z. Horvolgyi, Appl. Phys. Lett. 2006, 89.

[86] S. Reculusa, S. Ravaine, Chem. Mat. 2003, 15, 598-605.

[87] M. Marquez, B. P. Grady, Langmuir 2004, 20, 10998-11004.

[88] E. Sirotkin, J. D. Apweiler, F. Y. Ogrin, Langmuir 2010, 26, 10677-10683.

[89] F. Pan, J. Y. Zhang, C. Cai, T. M. Wang, Langmuir 2006, 22, 7101-7104.

[90] S. Jeong, L. B. Hu, H. R. Lee, E. Garnett, J. W. Choi, Y. Cui, Nano Lett. 2010, 10, 2989-2994.

[91] P. Kumnorkaew, Y.-K. Ee, N. Tansu, J. F. Gilchrist, Langmuir 2008, 24, 12150-12157.

[92] N. V. Dziomkina, M. A. Hempenius, G. J. Vancso, Adv. Mater. 2005, 17, 237.

[93] F. Burmeister, C. Schafle, T. Matthes, M. Bohmisch, J. Boneberg, P. Leiderer, Langmuir 1997, 13, 2983-2987.

[94] N. Vogel, S. Goerres, C. K. Weiss, K. Landfester, Macromol. Chem. Phys. 2011, accepted for publication.

[95] A. Plettl, F. Enderle, M. Saitner, A. Manzke, C. Pfahler, S. Wiedemann, P. Ziemann, Adv. Funct. Mater. 2009, 19, 3279-3284.

[96] X. Yan, J. Yao, G. Lu, X. Li, J. Zhang, K. Han, B. Yang, J. Am. Chem. Soc. 2005, 127, 7688-7689.

[97] X. Li, T. Q. Wang, J. H. Zhang, X. Yan, X. M. Zhang, D. F. Zhu, W. Li, X. Zhang, B. Yang, Langmuir 2010, 26, 2930-2936.

[98] S. Venkatesh, P. Jiang, B. Jiang, Langmuir 2007, 23, 8231-8235.

[99] T. S. Horozov, R. Aveyard, J. H. Clint, B. P. Binks, Langmuir 2003, 19, 2822-2829.

[100] T. S. Horozov, R. Aveyard, B. P. Binks, J. H. Clint, Langmuir 2005, 21, 7405-7412.

[101] M. A. Ray, N. Shewmon, S. Bhawalkar, L. Jia, Y. Yang, E. S. Daniels, Langmuir 2009, 25, 7265-7270.

[102] M. Retsch, K. H. Dostert, S. K. Nett, N. Vogel, J. S. Gutmann, U. Jonas, Soft Matter 2010, 6, 2403-2412.

[103] B. J. Park, E. M. Furst, Soft Matter 2010, 6, 485-488.

[104] K. P. Velikov, C. G. Christova, R. P. A. Dullens, A. van Blaaderen, Science 2002, 296, 106-109.

[105] M. H. Kim, S. H. Im, O. O. Park, Adv. Mater. 2005, 17, 2501.

[106] Z. C. Zhou, Q. F. Yan, Q. Li, X. S. Zhao, Langmuir 2007, 23, 1473-1477. 
[107] X. G. Huang, J. Zhou, M. Fu, B. Li, Y. H. Wang, Q. Zhao, Z. W. Yang, Q. Xie, L. T. Li, Langmuir 2007, 23, 8695-8698.

[108] K. W. Tan, G. Li, Y. K. Koh, Q. F. Yan, C. C. Wong, Langmuir 2008, 24, 9273-9278.

[109] D. Y. Wang, H. Mohwald, Adv. Mater. 2004, 16, 244.

[110] V. Kitaev, G. A. Ozin, Adv. Mater. 2003, 15, 75.

[111] H. L. Cong, W. X. Cao, J. Phys. Chem. B 2005, 109, 1695-1698.

[112] A. Sanchez-Iglesias, M. Grzelczak, J. Perez-Juste, L. M. Liz-Marzan, Angew. Chem.Int. Edit. 2010, 49, 9985-9989.

[113] P. Kumnorkaew, J. F. Gilchrist, Langmuir 2009, 25, 6070-6075.

[114] G. Singh, S. Pillai, A. Arpanaei, P. Kingshott, Adv. Mater. 2011, 23, 1519-1523.

[115] J. Yu, Q. F. Yan, D. Z. Shen, ACS Appl. Mater. Interfaces 2010, 2, 1922-1926.

[116] A. Detrich, A. Deak, E. Hild, A. L. Kovacs, Z. Horvolgyi, Langmuir 2010, 26, 26942699.

[117] L. Chi, Nanotechnology-Volume 8: Nanostructured Surfaces, Wiley-VCH, Weinheim, 2010.

[118] J.-Y. Wang, W. Chen, T. P. Rusell, Patterning with Block-Copolymers, Wiley, Hoboken, USA, 2009.

[119] J. P. Spatz, S. Mossmer, C. Hartmann, M. Moller, T. Herzog, M. Krieger, H. G. Boyen, P. Ziemann, B. Kabius, Langmuir 2000, 16, 407-415.

[120] G. Kastle, H. G. Boyen, F. Weigl, G. Lengl, T. Herzog, P. Ziemann, S. Riethmuller, O. Mayer, C. Hartmann, J. P. Spatz, M. Moller, M. Ozawa, F. Banhart, M. G. Garnier, P. Oelhafen, Adv. Funct. Mater. 2003, 13, 853-861.

[121] A. Manzke, C. Pfahler, O. Dubbers, A. Plettl, P. Ziemann, D. Crespy, E. Schreiber, U. Ziener, K. Landfester, Adv. Mater. 2007, 19, 1337.

[122] X. Y. Zhang, A. V. Whitney, J. Zhao, E. M. Hicks, R. P. Van Duyne, J. Nanosci. Nanotechnol. 2006, 6, 1920-1934.

[123] K. A. Willets, R. P. Van Duyne, Annu. Rev. Phys. Chem. 2007, 58, 267-297.

[124] J. N. Anker, W. P. Hall, O. Lyandres, N. C. Shah, J. Zhao, R. P. Van Duyne, Nat. Mater. 2008, 7, 442-453.

[125] C. L. Haynes, R. P. Van Duyne, J. Phys. Chem. B 2001, 105, 5599-5611.

[126] G. H. Chan, J. Zhao, E. M. Hicks, G. C. Schatz, R. P. Van Duyne, Nano Lett. 2007, 7, 1947-1952.

[127] P. Hanarp, M. Kall, D. S. Sutherland, J. Phys. Chem. B 2003, 107, 5768-5772.

[128] J. Aizpurua, P. Hanarp, D. S. Sutherland, M. Kall, G. W. Bryant, F. J. G. de Abajo, Phys. Rev. Lett. 2003, 90.

[129] J. S. Shumaker-Parry, H. Rochholz, M. Kreiter, Adv. Mater. 2005, 17, 2131.

[130] H. Rochholz, N. Bocchio, M. Kreiter, New J. Phys. 2007, 9.

[131] P. Jiang, M. J. McFarland, J. Am. Chem. Soc. 2005, 127, 3710-3711.

[132] F. Q. Sun, J. C. Yu, Angew. Chem.-Int. Edit. 2007, 46, 773-777.

[133] P. Jiang, Langmuir 2006, 22, 3955-3958.

[134] D. F. Liu, Y. J. Xiang, X. C. Wu, Z. X. Zhang, L. F. Liu, L. Song, X. W. Zhao, S. D. Luo, W. J. Ma, J. Shen, W. Y. Zhou, G. Wang, C. Y. Wang, S. S. Xie, Nano Lett. 2006, 6, 2375-2378.

[135] Y. Li, X. S. Fang, N. Koshizaki, T. Sasaki, L. Li, S. Y. Gao, Y. Shimizu, Y. Bando, D. Golberg, Adv. Funct. Mater. 2009, 19, 2467-2473.

[136] M. Retsch, M. Tamm, N. Bocchio, N. Horn, R. Forch, U. Jonas, M. Kreiter, Small 2009, 5, 2105-2110.

[137] A. Sinitskii, J. M. Tour, J. Am. Chem. Soc. 2010, 132, 14730-14732.

[138] K. C. Hsieh, T. Y. Tsai, D. H. Wan, H. L. Chen, N. H. Tai, ACS Nano 2010, 4, 13271336.

[139] Z. R. Taylor, K. Patel, T. G. Spain, J. C. Keay, J. D. Jernigen, E. S. Sanchez, B. P. Grady, M. B. Johnson, D. W. Schmidtke, Langmuir 2009, 25, 10932-10938.

[140] Y. F. Li, J. H. Zhang, S. J. Zhu, H. P. Dong, F. Jia, Z. H. Wang, Z. Q. Sun, L. Zhang, Y. Li, H. B. Li, W. Q. Xu, B. Yang, Adv. Mater. 2009, 21, 4731.

[141] F. Yan, W. A. Goedel, Chem. Mat. 2004, 16, 1622-1626. 
[142] G. Raschke, Ludwig Maximilians University (Munich), 2005.

[143] P. Drude, Ann. Phys.-Berlin 1900, 1, 566-613.

[144] S. A. Maier, Plasmonics - Fundamentals and Applications, Springer, New York, 2007.

[145] C. Sönnichsen, Plasmons in metal nanostructures, Cuviller Verlag, Goettingen, 2001.

[146] G. Mie, Ann. Phys.-Berlin 1908, 25, 377.

[147] I. Zins, Johannes Gutenberg University (Mainz, Germany), 2011.

[148] N. L. Bocchio, A. Unger, M. Alvarez, M. Kreiter, J. Phys. Chem. C 2008, 112, 1435514359.

[149] R. Contreras-Caceres, A. Sanchez-Iglesias, M. Karg, I. Pastoriza-Santos, J. Perez-Juste, J. Pacifico, T. Hellweg, A. Fernandez-Barbero, L. M. Liz-Marzan, Adv. Mater. 2008, 20, 1666.

[150] A. J. Haes, W. P. Hall, L. Chang, W. L. Klein, R. P. Van Duyne, Nano Lett. 2004, 4, 1029-1034.

[151] E. Prodan, C. Radloff, N. J. Halas, P. Nordlander, Science 2003, 302, 419-422.

[152] P. Nordlander, C. Oubre, E. Prodan, K. Li, M. I. Stockman, Nano Lett. 2004, 4, 899903.

[153] G. Binnig, C. F. Quate, C. Gerber, Phys. Rev. Lett. 1986, 56, 930-933.

[154] S. Greenfield, C. T. Berry, I. L. Jones, Analyst 1964, 89, 713.

[155] D. Perret, M. E. Newman, J. C. Negre, Y. W. Chen, J. Buffle, Water Res. 1994, 28, 91 106.

[156] A. Itoh, T. Nagasawa, Y. B. Zhu, K. H. Lee, E. Fujimori, H. Haraguchi, Anal. Sci. 2004, 20, 29-36.

[157] J. F. Ranville, D. J. Chittleborough, F. Shanks, R. J. S. Morrison, T. Harris, F. Doss, R. Beckett, Analytica Chimica Acta 1999, 381, 315-329.

[158] J. W. Olesik, Anal. Chem. 1991, 63, A12-A21.

[159] J. Nölte, ICP Emission Spectrometry, Wiley-VCH, Weinheim, Germany, 2002.

[160] R. H. Wendt, V. A. Fassel, Anal. Chem. 1965, 37, 920.

[161] C. Degueldre, P. Y. Favarger, S. Wold, Analytica Chimica Acta 2006, 555, 263-268.

[162] A. Scheffer, C. Engelhard, M. Sperling, W. Buscher, Anal. Bioanal. Chem. 2008, 390, 249-252.

[163] C. Degueldre, P. Y. Favarger, C. Bitea, Analytica Chimica Acta 2004, 518, 137-142.

[164] J. C. Farinas, R. Moreno, J. M. Mermet, J. Anal. At. Spectrom. 1994, 9, 841-849.

[165] C. Vancaeyzeele, O. Ornatsky, V. Baranov, L. Shen, A. Abdelrahman, M. A. Winnik, J. Am. Chem. Soc. 2007, 129, 13653-13660.

[166] I. Langmuir, J. Am. Chem. Soc. 1917, 39, 1848-1906.

[167] K. B. Blodgett, J. Am. Chem. Soc. 1934, 56, 495-495.

[168] K. B. Blodgett, J. Am. Chem. Soc. 1935, 57, 1007-1022.

[169] I. Langmuir, V. J. Schaefer, D. M. Wrinch, Science 1937, 85, 76-80.

[170] M. Knoll, Z. Tech. Phys. 1935, 16; 36, 467; 861-475; 869.

[171] J. I. Goldstein, D. E. Newbury, P. Echlin, D. C. KJoy, A. D. Romig, A. E. Lymann, C. Fiori, E. Lifshin, Scanning Electron Microscopy and X-Ray Microanalysis, Plenum Press, New York, 1992.

[172] B. Vonnegut, Rev. Sci. Instrum. 1942, 13, 6-9.

[173] A. Couper, R. Newton, C. Nunn, Colloid Polym. Sci. 1983, 261, 371-372.

[174] N. Vogel, C. P. Hauser, K. Schuller, K. Landfester, C. K. Weiss, Macromol. Chem. Phys. 2010, 211, 1355-1368.

[175] S. Utech, C. Scherer, M. Maskos, J. Magn. Magn. Mater. 2008, 321, 1386-1388.

[176] Y. H. Yang, Z. K. Wen, Y. P. Dong, M. Y. Gao, Small 2006, 2, 898-901.

[177] J. W. Olesik, A. W. Moore, Anal. Chem. 1990, 62, 840-845.

[178] M. W. Routh, Spectrochimica Acta Part B: Atomic Spectroscopy 1986, 41, 39-48.

[179] J. M. Mermet, J. Anal. At. Spectrom. 1998, 13, 419-422.

[180] C. Dubuisson, E. Poussel, J. L. Todoli, J. M. Mermet, Spectroc. Acta Pt. B-Atom. Spectr. 1998, 53, 593-600.

[181] N. Vogel, U. Ziener, A. Manzke, A. Plettl, P. Ziemann, J. Biskupek, C. K. Weiss, K. Landfester, Beilstein Journal of Nanotechnology 2011. 
[182] C. van den Brom, N. Vogel, C. P. Hauser, S. Goerres, M. Wagner, K. Landfester, C. K. Weiss, Langmuir 2011, accepted for publication.

[183] M. Egen, R. Zentel, Macromol. Chem. Phys. 2004, 205, 1479-1488.

[184] C. Lopez, Adv. Mater. 2003, 15, 1679-1704.

[185] F. Marlow, Muldarisnur, P. Sharifi, R. Brinkmann, C. Mendive, Angew. Chem. Int. Ed. 2009, 48, 6212-6233.

[186] T. Still, W. Cheng, M. Retsch, R. Sainidou, J. Wang, U. Jonas, N. Stefanou, G. Fytas, Phys. Rev. Lett. 2008, 100.

[187] T. Still, W. Cheng, M. Retsch, U. Jonas, G. Fytas, J. Phys., Condens. Matter. 2008, 20, 404203.

[188] N. Vogel, M. Jung, M. Retsch, W. Knoll, U. Jonas, I. Koper, Small 2009, 5, 821-825.

[189] V. M. Nekipelov, K. I. Zamaraev, Coord. Chem. Rev. 1985, 61, 185-240.

[190] J. E. Huheey, E. A. Keiter, R. L. Keiter, Anorganische Chemie: Prinzipien von Struktur und Reaktivität, Walter de Gruyter, Berlin, 1995.

[191] D. Steinborn, S. Schwieger, Chem.-Eur. J. 2007, 13, 9668-9678.

[192] M. H. Abraham, J. A. Platts, J. Org. Chem. 2001, 66, 3484-3491.

[193] N. Wiberg, A. F. Holleman, E. Wiberg, Holleman-Wiberg's Inorganic Chemisry, 101st ed. ed., Academic Press, San Diego, CA, USA, 2002.

[194] A. Al-Alousy, J. Burgess, Polyhedron 1992, 11, 531-539.

[195] E. M. Coen, R. G. Gilbert, B. R. Morrison, H. Leube, S. Peach, Polymer 1998, 39, 7099-7112.

[196] I. A. Maxwell, B. R. Morrison, D. H. Napper, R. G. Gilbert, Macromolecules 1991, 24, 1629-1640.

[197] B. R. Morrison, R. G. Gilbert, Macromol. Symp. 1995, 92, 13-30.

[198] S. Jiang, E. D. Sudol, V. L. Dimonie, M. S. El-Aasser, J. Appl. Polym. Sci. 2008, 108, 4096-4107.

[199] C. S. Chern, T. J. Chen, S. Y. Wu, H. B. Chu, C. F. Huang, J. Macromol. Sci.-Pure Appl. Chem. 1997, A34, 1221-1236.

[200] J. E. Jonsson, H. Hassander, B. Tornell, Macromolecules 1994, 27, 1932-1937.

[201] V. Castelvetro, C. De Vita, G. Giannini, S. Giaiacopi, J. Appl. Polym. Sci. 2006, 102, 3083-3094.

[202] S. Reynaert, P. Moldenaers, J. Vermant, Langmuir 2006, 22, 4936-4945.

[203] M. A. Ray, L. Jia, Adv. Mater. 2007, 19, 2020.

[204] S. P. Bhawalkar, J. Qian, M. C. Heiber, L. Jia, Langmuir 2010, 26, 16662-16666.

[205] D. G. Choi, H. K. Yu, S. G. Jang, S. M. Yang, J. Am. Chem. Soc. 2004, 126, 70197025 .

[206] T. M. Blattler, A. Binkert, M. Zimmermann, M. Textor, J. Voros, E. Reimhult, Nanotechnology 2008, 19.

[207] J. W. Coburn, H. F. Winters, Journal of Vacuum Science \& Technology 1979, 16, 391403.

[208] D. L. Flamm, V. M. Donnelly, D. E. Ibbotson, J. Vac. Sci. Technol. B 1983, 1, 23-30.

[209] Y. H. Ting, S. M. Park, C. C. Liu, X. S. Liu, F. J. Himpsel, P. F. Nealey, A. E. Wendt, J. Vac. Sci. Technol. B 2008, 26, 1684-1689.

[210] S. J. Moss, A. M. Jolly, B. J. Tighe, Plasma Chem. Plasma Process. 1986, 6, 401-416.

[211] R. M. France, R. D. Short, Langmuir 1998, 14, 4827-4835.

[212] N. Vogel, J. Fischer, R. Mohammadi, M. Retsch, H. J. Butt, K. Landfester, C. K. Weiss, M. Kreiter, Nano Lett. 2011, 11, 446-454.

[213] Y. B. Zheng, B. K. Juluri, X. L. Mao, T. R. Walker, T. J. Huang, J. Appl. Phys. 2008, 103.

[214] C. Werdinius, L. Osterlund, B. Kasemo, Langmuir 2003, 19, 458-468.

[215] S. Guruvenket, G. M. Rao, M. Komath, A. M. Raichur, Appl. Surf. Sci. 2004, 236, $278-$ 284.

[216] C. D. Dushkin, K. Nagayama, T. Miwa, P. A. Kralchevsky, Langmuir 1993, 9, 36953701 .

[217] A. S. Dimitrov, T. Miwa, K. Nagayama, Langmuir 1999, 15, 5257-5264. 
[218] M. Born, E. Wolf, Principles of Optics, 4th ed., Pergamon Press; Oxford, 1970.

[219] J. W. Goodwin, R. H. Ottewill, A. Parentich, J. Phys. Chem. 1980, 84, 1580-1586.

[220] E. V. Shevchenko, D. V. Talapin, N. A. Kotov, S. O'Brien, C. B. Murray, Nature 2006, 439, 55-59.

[221] D. V. Talapin, E. V. Shevchenko, M. I. Bodnarchuk, X. Ye, J. Chen, C. B. Murray, Nature 2009, 461, 964-967.

[222] Y. Wan, Z. Y. Cai, L. H. Xia, L. K. Wang, Y. Q. Li, Q. Li, X. S. Zhao, Mater. Lett. 2009, 63, 2078-2081.

[223] Y. D. Yin, Y. Lu, B. Gates, Y. N. Xia, J. Am. Chem. Soc. 2001, 123, 8718-8729.

[224] A. Musyanovych, R. Rossmanith, C. Tontsch, K. Landfester, Langmuir 2007, 23, 53675376.

[225] A. Ethirajan, K. Landfester, Chem.-Eur. J. 2010, 16, 9398-9412.

[226] J. Tang, S. Daniels Eric, L. Dimonie Victoria, A. Klein, S. El-Aasser Mohamed, in Film Formation in Coatings, Vol. 790, American Chemical Society, 2001, pp. 212-232.

[227] A. Zeller, A. Musyanovych, M. Kappl, A. Ethirajan, M. Dass, D. Markova, M. Klapper, K. Landfester, ACS Appl. Mater. Interfaces 2010, 2, 2421-2428.

[228] J. B. Lassiter, H. Sobhani, J. A. Fan, J. Kundu, F. Capasso, P. Nordlander, N. J. Halas, Nano Lett 2010, 10, 3184-3189.

[229] J. A. Fan, C. H. Wu, K. Bao, J. M. Bao, R. Bardhan, N. J. Halas, V. N. Manoharan, P. Nordlander, G. Shvets, F. Capasso, Science 2010, 328, 1135-1138.

[230] A. Manzke, N. Vogel, C. K. Weiss, U. Ziener, A. Plettl, K. Landfester, P. Ziemann, Nanoscale 2011, 3, 2523.

[231] A. Manzke, A. Plettl, U. Wiedwald, L. Han, P. Ziemann, E. Schreiber, U. Ziener, N. Vogel, K. Landfester, K. Fauth, J. Biskupek, U. Kaiser, 2011, submitted.

[232] B. R. Cuenya, Thin Solid Films 2010, 518, 3127-3150.

[233] M. H. Lee, M. D. Huntington, W. Zhou, J.-C. Yang, T. W. Odom, Nano Lett. 2010, 11, 311-315.

[234] D. Aydin, M. Schwieder, I. Louban, S. Knoppe, J. Ulmer, T. L. Haas, H. Walczak, J. P. Spatz, Small 2009, 5, 1014-1018.

[235] J. Huang, S. V. Gräter, F. Corbellini, S. Rinck, E. Bock, R. Kemkemer, H. Kessler, J. Ding, J. P. Spatz, Nano Lett. 2009, 9, 1111-1116.

[236] J. Lyubina, B. Rellinghaus, O. Gutfleisch, M. Albrecht, in Handbook of Magnetic Materials, Vol. 19 (Ed.: K. H. Buschow), Elsevier, Amsterdam, 2011.

[237] H. Kobayashi, T. Yuasa, K. Yamanaka, K. Yoneda, Y. Todokoro, J. Chem. Phys. 1998, 109, 4997-5001.

[238] A. Unger, N. Bocchio, N. Vogel, M. Retsch, M. Tamm, M. Kreiter, submitted.

[239] J. Fischer, N. Vogel, R. Mohammadi, H. J. Butt, K. Landfester, C. K. Weiss, M. Kreiter, 2011, submitted.

[240] W. A. Murray, W. L. Barnes, Adv. Mater. 2007, 19, 3771-3782.

[241] J. B. Pendry, Contemp. Phys. 2004, 45, 191-202.

[242] R. A. Shelby, D. R. Smith, S. Schultz, Science 2001, 292, 77-79.

[243] D. R. Smith, J. B. Pendry, M. C. K. Wiltshire, Science 2004, 305, 788-792.

[244] J. B. Pendry, D. Schurig, D. R. Smith, Science 2006, 312, 1780-1782.

[245] D. Schurig, J. J. Mock, B. J. Justice, S. A. Cummer, J. B. Pendry, A. F. Starr, D. R. Smith, Science 2006, 314, 977-980.

[246] W. S. Cai, U. K. Chettiar, A. V. Kildishev, V. M. Shalaev, Nat. Photonics 2007, 1, 224227.

[247] N. Liu, H. C. Guo, L. W. Fu, S. Kaiser, H. Schweizer, H. Giessen, Nat. Mater. 2008, 7, 31-37.

[248] N. Liu, H. Liu, S. N. Zhu, H. Giessen, Nat. Photonics 2009, 3, 157-162.

[249] C. M. Soukoulis, S. Linden, M. Wegener, Science 2007, 315, 47-49.

[250] V. M. Shalaev, Nat. Photonics 2007, 1, 41-48.

[251] V. M. Shalaev, W. S. Cai, U. K. Chettiar, H. K. Yuan, A. K. Sarychev, V. P. Drachev, A. V. Kildishev, Opt. Lett. 2005, 30, 3356-3358. 
[252] M. C. Gwinner, E. Koroknay, L. W. Fu, P. Patoka, W. Kandulski, M. Giersig, H. Giessen, Small 2009, 5, 400-406.

[253] N. Liu, H. C. Guo, L. W. Fu, S. Kaiser, H. Schweizer, H. Giessen, Adv. Mater. 2007, $19,3628$.

[254] H. C. Guo, N. Liu, L. W. Fu, T. P. Meyrath, T. Zentgraf, H. Schweizer, H. Giessen, Opt. Express 2007, 15, 12095-12101.

[255] S. Sheikholeslami, Y.-w. Jun, P. K. Jain, A. P. Alivisatos, Nano Lett. 2010, 10, 26552660.

[256] J. Pomplun, S. Burger, L. Zschiedrich, F. Schmidt, Phys. Status Solidi B-Basic Solid State Phys. 2007, 244, 3419-3434.

[257] P. B. Johnson, R. W. Christy, Phys. Rev. B 1972, 6, 4370-4379.

[258] B. Auguie, W. L. Barnes, Opt. Lett. 2009, 34, 401-403.

[259] A. Unger, U. Rietzler, R. d. Berger, M. Kreiter, Nano Lett. 2009, 9, 2311-2315.

[260] J. Fischer, N. Bocchio, A. Unger, H.-J. r. Butt, K. Koynov, M. Kreiter, The Journal of Physical Chemistry C 2010, 114, 20968-20973.

[261] Y. Choi, Y. Park, T. Kang, L. P. Lee, Nat Nano 2009, 4, 742-746.

[262] N. Vogel, Diploma Thesis thesis, Johannes Gutenberg University (Mainz), 2008.

[263] M. Jung, Dissertation thesis, Johannes Gutenberg University (Mainz), 2008.

[264] N. Vogel, M. Jung, N. L. Bocchio, M. Retsch, M. Kreiter, I. Koper, Small 2010, 6, 104109.

[265] M. Jung, N. Vogel, I. Koeper, Langmuir 2011, 27, 7008.

[266] H. J. Butt, D. N. Wang, P. K. Hansma, W. Kuhlbrandt, Ultramicroscopy 1991, 36, 307318.

[267] H. J. Butt, T. Muller, H. Gross, J. Struct. Biol. 1993, 110, 127-132.

[268] M. Hegner, P. Wagner, G. Semenza, Surf. Sci. 1993, 291, 39-46.

[269] P. Wagner, M. Hegner, H. J. Guntherodt, G. Semenza, Langmuir 1995, 11, 3867-3875.

[270] S. M. Schiller, R. Naumann, K. Lovejoy, H. Kunz, W. Knoll, Angew. Chem.-Int. Edit. 2003, 42, 208.

[271] R. Naumann, S. M. Schiller, F. Giess, B. Grohe, K. B. Hartman, I. Karcher, I. Koper, J. Lubben, K. Vasilev, W. Knoll, Langmuir 2003, 19, 5435-5443.

[272] I. K. Vockenroth, P. P. Atanasova, A. T. A. Jenkins, I. Koper, Langmuir 2008, 24, 496502.

[273] X. L. Zhu, Y. Zhang, J. S. Zhang, J. Xu, Y. Ma, Z. Y. Li, D. P. Yu, Adv. Mater. 2010, 22, 4345.

[274] P. Nagpal, N. C. Lindquist, S. H. Oh, D. J. Norris, Science 2009, 325, 594-597.

[275] Y. Zhou, K. P. Loh, Adv. Mater. 2010, 22, 3615-3620.

[276] Z. Xiaoyu, A. V. Whitney, Z. Jing, E. M. Hicks, R. P. Van Duyne, J. Nanosci. Nanotechnol. 2006, 6, 1920-1934.

[277] A. J. Haes, J. Zhao, S. L. Zou, C. S. Own, L. D. Marks, G. C. Schatz, R. P. Van Duyne, J. Phys. Chem. B 2005, 109, 11158-11162.

[278] J. C. Riboh, A. J. Haes, A. D. McFarland, C. R. Yonzon, R. P. Van Duyne, J. Phys. Chem. B 2003, 107, 1772-1780.

[279] E. M. Hicks, O. Lyandres, W. P. Hall, S. L. Zou, M. R. Glucksberg, R. P. Van Duyne, J. Phys. Chem. C 2007, 111, 4116-4124.

[280] M. D. Malinsky, K. L. Kelly, G. C. Schatz, R. P. Van Duyne, J. Am. Chem. Soc. 2001, 123, 1471-1482.

[281] G. Decher, Science 1997, 277, 1232-1237.

[282] G. Decher, J. D. Hong, J. Schmitt, Thin Solid Films 1992, 210, 831-835.

[283] K. Aikawa, H. Sakata, S. Furuuchi, J. Mater. Sci. 1978, 13, 37-42.

[284] I. Koper, Molecular BioSystems 2007, 3, 651-657.

[285] E. Sackmann, Science 1996, 271, 43-48.

[286] Y.-H. M. Chan, S. G. Boxer, Current Opinion in Structural Biology 2007, 11, 581-587.

[287] E. K. Sinner, S. Ritz, Y. Wang, J. Dostalek, U. Jonase, W. Knoll, Mater. Today 2010, 13, 46-55. 
[288] R. Robelek, E. S. Lemker, B. Wiltschi, V. Kirste, R. Naumann, D. Oesterhelt, E. K. Sinner, Angew. Chem.-Int. Edit. 2007, 46, 605-608.

[289] I. Köper, Molecular Biosystems 2007, 3, 651.

[290] V. Atanasov, P. P. Atanasova, I. K. Vockenroth, N. Knorr, I. Koper, Bioconjugate Chem. 2006, 17, 631-637.

[291] K. Morigaki, T. Baumgart, U. Jonas, A. Offenhausser, W. Knoll, Langmuir 2002, 18, 4082-4089.

[292] K. Morigaki, K. Kiyosue, T. Taguchi, Langmuir 2004, 20, 7729-7735.

[293] J. S. Hovis, S. G. Boxer, Langmuir 2000, 16, 894-897.

[294] J. T. Groves, N. Ulman, S. G. Boxer, Science 1997, 275, 651-653.

[295] A. M. Smith, M. Vinchurkar, N. Gronbech-Jensen, A. N. Parikh, J. Am. Chem. Soc., $132,9320-9327$.

[296] R. Naumann, S. M. Schiller, F. Giess, B. Grohe, K. B. Hartman, I. Kärcher, I. Köper, J. Lübben, K. Vasilev, W. Knoll, Langmuir 2003, 19, 5435-5443.

[297] D. M. Soumpasis, Biophys. J. 1983, 41, 95-97.

[298] A. Kusumi, C. Nakada, K. Ritchie, K. Murase, K. Suzuki, H. Murakoshi, R. S. Kasai, J. Kondo, T. Fujiwara, Annual Review of Biophysics and Biomolecular Structure 2005, 34, 351-378.

[299] C. Dietrich, Z. N. Volovyk, M. Levi, N. L. Thompson, K. Jacobson, Proc. Natl. Acad. Sci. U. S. A. 2001, 98, 10642-10647.

[300] J. C. Mcintyre, R. G. Sleight, Biochemistry-Us 1991, 30, 11819-11827. 